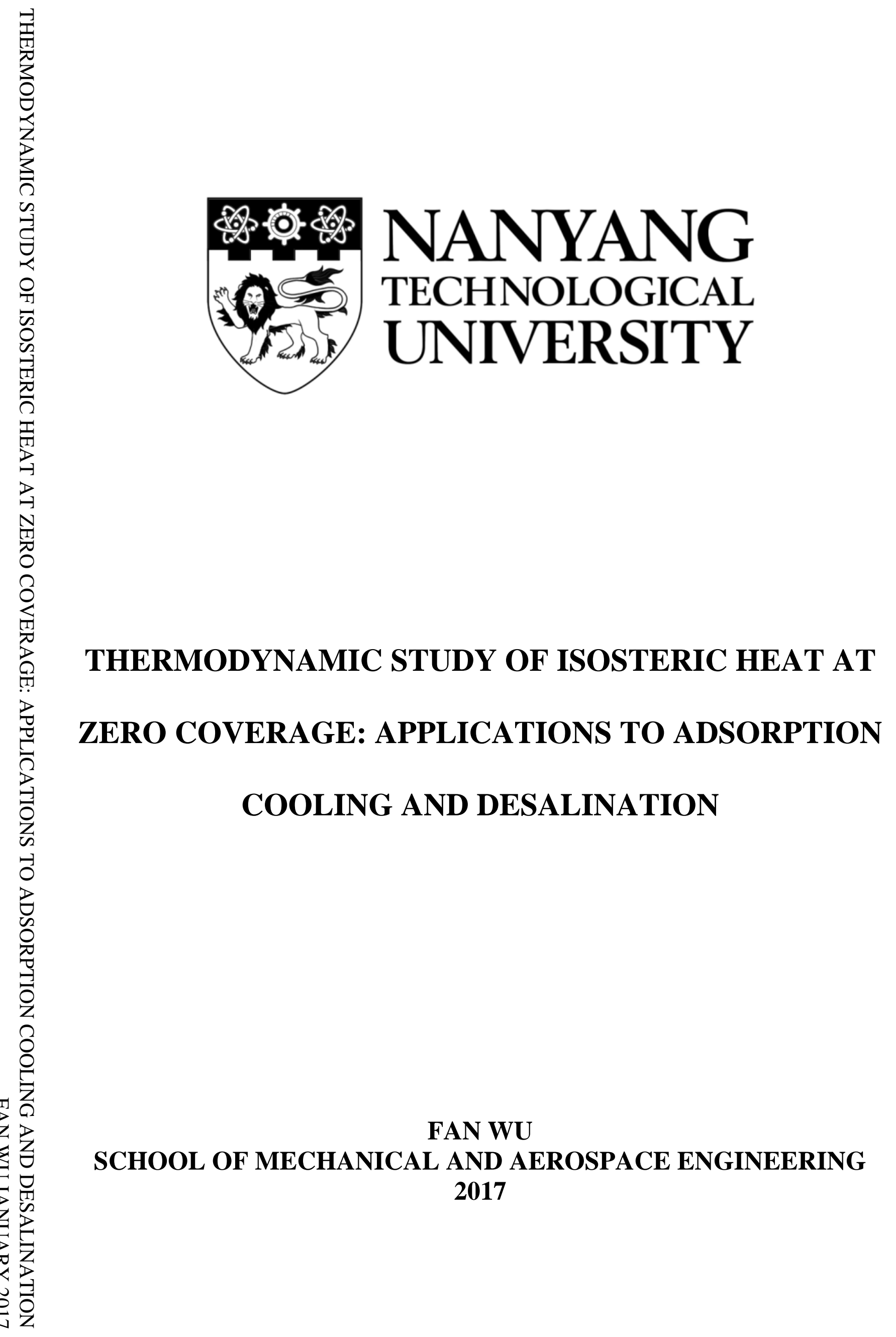




\section{THERMODYNAMIC STUDY OF ISOSTERIC HEAT AT ZERO COVERAGE: APPLICATIONS TO ADSORPTION COOLING AND DESALINATION}

School of Mechanical and Aerospace Engineering

A thesis submitted to Nanyang Technological University in partial fulfillment of the requirements for the degree of DOCTOR OF PHILOSOPHY 


\begin{abstract}
The production of cooling and drinkable water represents major and essential needs for the mankind. Vapor compression cooling and desalination systems are mainly driven by electricity, produced by burning fossil fuels, which contributes to the global warming $(\mathrm{GW})$ because of the encountered direct $\mathrm{CO}_{2}$-emissions. The refrigerants used in vapor compression systems possess high global warming potential (GWP), resulting in an indirect contribution to the GW and, consequently, to the severe consequences on the worldwide climate. Recovering the waste heat generated upon burning fossil fuels to provide both cooling and fresh water demands through the application of adsorption assisted cooling and desalination cycle may remarkably contribute in mitigating the bad consequences of the GW.
\end{abstract}

At present, the bulky size of adsorption chiller and desalination systems obstructs its practical application. The size of an adsorption bed depends on the quality of the porous adsorbents. Therefore, the present thesis aims to develop a thermodynamic framework for calculating the interaction potentials between the adsorbate and the adsorbent pore structures, from which the isosteric heat at zero coverage is formulated as a function of pore width and volume. The proposed modelling provides necessary information for the design of adsorbent materials.

The proposed interaction potential model includes the Lennard Jones (LJ) Potential with the inclusion of electrostatic and induction potential. The model is first applied to the graphite surface, in which the electrostatic and induction potentials are obtained to be very small compared to the LJ potential. The maximum isosteric heat is found in the super micro-pore regions. On the other hand, the Reverse Monte Carlo (RMC) and molecular dynamics methods are applied to understand the silica structure for water adsorption. For water interaction with silica gel, the electrostatic interaction is found to be high. For water adsorption on CHA and AFI 
types zeolites, the most favourable adsorptive sites are identified at the positions of channels inside the zeolite frameworks.

A detailed experimental investigation is conducted for measuring (i) the amount of $\mathrm{CO}_{2}$ uptakes on various activated carbons, and (ii) water uptakes on various types of silica gels and zeolites under static conditions. These isotherms data are fitted with isotherm models for their use in adsorption cooling and desalination modelling.

Employing the knowledge of isosteric heats, pore structures and isotherm data, a thermodynamic model is developed for calculating the energetic performances of adsorption cooling and desalination systems. From the present analysis, it is noticed that both graphite structure or activated carbon and silica gels are optimised with respect to pore sizes and volumes for given evaporating and heat source temperatures. The best performances of adsorption cooling and desalination cycles are obtained from a compromise between the greatest pore volume and the optimal pore size. From the present analysis, it is observed that there is no cooling and water production performance for pore widths varying from 0 to $0.3 \mathrm{~nm}$. For better efficiency, the silica gel should be designed with a pore size from $1 \mathrm{~nm}$ to $2 \mathrm{~nm}$ for cooling application due to its higher coefficient of performance (COP). The average pore size of silica gel should be designed with $1 \mathrm{~nm}$ for the production of more water. On the other hand, for refrigeration purposes, the activated carbon should be designed with the average pore size ranging from 7 to $15 \AA$ and higher micro-pore volumes.

This thesis thus provides some important information regarding the knowledge of isosteric heat at zero coverage for various adsorbent - adsorbate pairs, which can help in the design of new carbonaceous materials or carbon-silica or zeolites composites for adsorption assisted cooling and desalination purposes. 


\section{Acknowledgements}

I appreciate my supervisor, Associate Professor Anutosh Chakraborty, for his pedagogical thoughtfulness and helpful guidance during the study and research work. Professor Chakraborty has spent a lot of time and effort in instructing me for the theoretical and experimental investigations as well as offering all the support that are required for the accomplishment of this thesis.

I would also thank Dr. Sun Baichuan and Mr. Harendra Kishor Karn for sharing the knowledge and the improvement of the experimental setup with investigations. They have always engaged me in discussions related to theory and experiment and their efforts are deeply appreciated.

Next, I would like to thank Mr. Syed Muztuza Ali for his help on discussions, dedication and support for the project. I also want to thank Dr. Sibnath Kayal and Mr. Teo How Wei Benjamin for their help for the research work.

Next, I would like to thank the technical staff of Energy Systems Laboratory of the school of Mechanical and Aerospace Engineering of Nanyang Technological University, Mr. Lee Keng Yuen and Mr. Ang Koon Teck Lawrence for their support during the research. I would like to thank particularly Mr. Yeo Boon Chuan Edward, who kindly spare no effort to support the research work all the way. I would also like to thank for the financial support from National Research Foundation, Singapore (Grant No. NRFCRP8- 2011-3).

My parents, sister, fiancée and friends have continuously supported me to pursue the research with love, care and help and I want to sincerely express my gratitude to them finally.

on New Year's Eve in Yunnan Garden, NTU 


\section{Publication Arising from This Thesis}

The author is grateful to his supervisor, Associate Professor Anutosh Chakraborty, who is a coauthor for the following publications

\section{Journal Papers}

1. Fan, W., Chakraborty, A., Kayal, S. Adsorption cooling cycles: Insights into carbon dioxide adsorption on activated carbons. Energy. 2016, 102: 491-501.

2. Fan, W., Chakraborty, A. Investigation of the Interaction of Polar Molecules on Graphite Surface: Prediction of Isosteric Heat of Adsorption at Zero Surface Coverage. Journal of Physical Chemistry C. 2016, 120 (41): 23490-23499.

3. Sun, B., Fan, W., Chakraborty, A. Adsorption Kinetics Emulation with Lattice Gas Cellular. Heat Transfer Engineering. 2017, 38(4): 409-416.

4. Teo, H.W., Chakraborty, A., Fan, W. Improved adsorption characteristics data for AQSOA types zeolites and water systems under static and dynamic conditions. Microporous and Mesoporous Materials. 2017, 242: 109-117.

\section{Conference Papers}

1. Chakraborty, A., Sun, B., Ali, S.M., Fan, W. Design and Development Of Cryo-Adsorption Chamber for the Measurement of Methane Uptakes on Activated Carbons and MOFs. Proceedings of the 7th Asian Conference on Refrigeration and Air Conditioning, Jeju, Korea, 2014.

2. Sun, B., Fan, W., Chakraborty, A., Adsorption Characteristics of Various MOFs for Gas Storage Applications. The 6th International Meeting on Advanced Thermofluids, Singapore, 2013. 


\section{List of Figures}

Figure 1.1 The schematic adsorption process ............................................................ 1

Figure 1.2 IUPAC Classifications of Adsorption Isotherms............................................... 3

Figure 1.3 The schematic formalism of adsorption isosteric heat ....................................... 4

Figure 2.1 Schematic of adsorption cooling and desalination system .................................. 14

Figure 3.1 The simulation model of graphite and water molecule (here $\mathrm{H}_{2} \mathrm{O}$ is considered as an example of polar molecule). The edge length of the hexagon of carbon atoms is $d=1.421 \AA$ and the distance of two layers is $\mathrm{I}=3.354 \AA$. The position vector points from the $\mathrm{i}^{\text {th }}$ carbon to the $\mathrm{j}^{\text {th }}$ atom of the adsorbate molecule. Position vector in adsorbate molecule coordinate system $X^{\prime} Y^{\prime}$ $Z^{\prime}$ can be converted to graphite coordinate system $X Y Z$ with a specific rotational matrix and

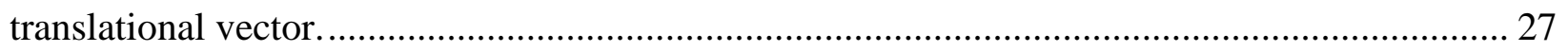

Figure 3.2 Electrostatic model of water and graphite. Here the dipole moment vector $(\mu)$ for water and quadrupole moment matrix $(\Theta)$ for water and carbon are shown. $\boldsymbol{r}_{i j}$ is the position vector from the ith carbon atom to the jth atom of water molecule and $\boldsymbol{r}_{i j}=-\boldsymbol{r}_{j i}$. The positive charge for hydrogen atom is $\mathrm{q}_{\mathrm{H}}$ and the negative charge of oxygen atom is $-2 \mathrm{q}_{\mathrm{H}}$. The electric field generated by the quadrupole moment of carbon atom at position $\boldsymbol{r}_{i j}$ is $\mathrm{E} \theta_{\mathrm{C}}\left(\boldsymbol{r}_{i j}\right)$ and the electric filed generated by the dipole and quadrupole moment of water is $\mathrm{E} \mu_{H 2 O} \theta_{H 2 O}\left(\boldsymbol{r}_{i j}\right)$ and $\theta_{H 2 O}$ are the quadrupole moment of carbon and water. $\mu_{H 2 O}$ and $\mu_{H 2 O}^{I}$ are the permanent and transient induced dipole moment of water.

Figure 3.3 The parallel slit shape pore model for graphite. The distance of the centre of adsorbate molecule to the surface of multi-layer graphite atoms and the opposing wall are $z$ and $H-z$ respectively for the pore width of $H$. The external wall potential of the adsorbate molecule in the pore is the sum of the interaction potentials with both walls. 31 
Figure 3.4 Radial distribution function of the $\mathrm{SiO}_{2}$ structure: (a) total radial distribution (b)

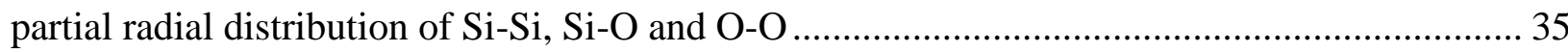

Figure 3.5 Comparison of O-Si-O and $\mathrm{Si}-\mathrm{O}-\mathrm{Si}$ angle distribution function of the $\mathrm{SiO}_{2}$ structure36 Figure 3.6 Total structure factor of the amorphous silica structure from RMC and XRD 37

Figure 3.7 The simulation model of silica gel and water molecule. The edge length of the simulation box is $\mathrm{a}=\mathrm{b}=\mathrm{c}=35.7 \AA$. The position vector $\left(\boldsymbol{r}_{i j}=\boldsymbol{r}_{i}-\boldsymbol{r}_{j}\right)$ points from the $\mathrm{j}^{\text {th }}$ atom (Si or $\mathrm{O})$ of adsorbent to the $\mathrm{i}^{\text {th }}$ atom $(\mathrm{H}$ or $\mathrm{O})$ of the water molecule, where $\boldsymbol{r}_{\boldsymbol{i}}$ is the position vector of $\mathrm{i}^{\text {th }}$ atom of water molecule, $\boldsymbol{r}_{j}$ is the position vector of $\mathrm{j}^{\text {th }}$ silicon/oxygen atom. The position vector in water molecule coordinate system $X^{\prime} \quad Y^{\prime} Z^{\prime}$ can be converted to the silica coordinate system $X Y Z$ with a specific rotational matrix and translational vector. 38

Figure 3.8 The model for calculating the average external wall potential as a function of $z$ for $\mathrm{SiO}_{2}$-Water system. The distance of the centre of water molecule to the surface of silica and the opposing wall are $z$ and $H-z$, respectively given the pore width of $\mathrm{H}$. The external wall potential of the adsorbate molecule in the pore is the sum of interaction potential with both walls. 39

Figure 3.9 Electrostatic interaction between $\mathrm{SiO}_{2}$ and $\mathrm{H}_{2} \mathrm{O}$ molecules. The $i^{\text {th }}$ atom of $\mathrm{SiO}_{2}$ molecule with the electrical charge of $\mathrm{q}_{\mathrm{Si}}$ or $\mathrm{q}_{\mathrm{O}}$ interacts with the $j^{\text {th }}$ atom of $\mathrm{H}_{2} \mathrm{O}$ molecule with the electrical charge of $q_{H}$ or $q_{O}$. The position vector $\boldsymbol{r}_{i j}$ characterises the distance between the interaction pair.

Figure 3.10 The interactions of water molecule with (a) CHA type and (b) AFI type zeolites according to cylindrical pore model. Hence 1, 2, 3 and 4 indicate the layers of cylindrical channels; $\mathrm{C}$ is the center of the channels, $\mathrm{r}$ is the distance of one water molecule from the center C. 
Figure 4.1 Five different orientations of a water molecule on the graphite surface at the distance of $\mathrm{z}$ in $\mathrm{Z}$ direction. 52

Figure 4.2 Interaction energy of a water molecule with the graphite surface as a function of C...O separation $(\mathrm{z})$ for five different orientations. 53

Figure 4.3 Three different orientations of a methanol molecule on the graphite surface at the distance of $\mathrm{z}$ in $\mathrm{Z}$ direction 54

Figure 4.4 Interaction energy of a methanol molecule with the graphite surface as a function of $\mathrm{C} \cdots \mathrm{CH}_{3} \mathrm{OH}$ separation $(\mathrm{z})$ for five different orientations. 55

Figure 4.5 Three different orientations of an ethanol molecule on the graphite surface at the distance of $\mathrm{z}$ in $\mathrm{Z}$ direction...... 56

Figure 4.6 Interaction energy of an ethanol molecule with the graphite surface as a function of $\mathrm{C} \cdots \mathrm{CH}_{3} \mathrm{CH}_{2} \mathrm{OH}$ separation (z) for five different orientation 56

Figure 4.7 A detailed analysis on interaction potentials $\mathrm{v}\left(\mathrm{z}_{\mathrm{o}}\right)$ for four polar molecules, where the orientations of $\mathrm{H}_{2} \mathrm{O}, \mathrm{CH}_{3} \mathrm{OH}$ and $\mathrm{CH}_{3} \mathrm{CH}_{2} \mathrm{OH}$ are chosen at their maximum $q_{s t}^{o}$ and $\mathrm{z}_{0}$. 57 Figure 4.8 Five orientations of water molecule above top surface of amorphous silica............. 58 Figure 4.9 Interaction potential of water molecule of five orientations with the amorphous silica structure, where electrostatic potential is found significantly very high compared with LJ potential. 60

Figure 4.10 Interaction potential of water molecule in orientation 1 with the amorphous silica structure. 61

Figure 4.11 Illustration of choosing five positions of water in orientation 1 on the amorphous silica structure 62 
Figure 4.12 Interaction potential of water molecule of five positions in orientation 1 with the amorphous silica structure

Figure 4.13 Interaction potential of water molecule at orientation 5 with distance of $2.15 \AA$, $2.65 \AA, 3.15 \AA$, and $3.65 \AA$ to the amorphous silica structure. The white portion indicates here the positive interaction potential. 64

Figure 4.14 Interaction potential for the adsorption of water molecules on CHA zeolite employing (a) a 3 dimensional, and (b) contour plots. 65

Figure 4.15 Interaction potentials of water molecule on AFI zeolite employing (a) a three dimensional, and ( b) contour plots. 66

Figure 4.16 The interaction potential of a water molecule with the cylindrical channel inside CHA zeotype as a function of distance to the centre of the channel. 67

Figure 4.17 The interaction potential of a water molecule with the cylindrical channel inside AFI zeotype as a function of distance to the centre of the channel. 68

Figure 4.18 Isosteric heat of adsorption of single water molecule on graphite lattice at zero surface coverage as a function of $\mathrm{z}$ for the selected five $\mathrm{H}_{2} \mathrm{O}$ orientations

Figure 4.19 Isosteric heat of adsorption of single $\mathrm{CH}_{3}-\mathrm{OH}$ molecule on graphite lattice at zero surface coverage as a function of $\mathrm{z}$ for the selected three $\mathrm{CH}_{3}-\mathrm{OH}$ orientations..... 70

Figure 4.20 Isosteric heat of adsorption of single ethanol molecule on graphite lattice at zero surface coverage as a function of $\mathrm{z}$ for the selected three $\mathrm{CH}_{3} \mathrm{CH}_{2} \mathrm{OH}$ orientations ..... 72 Figure 4.21 qstoof water of position 1 5 of orientation 1 on amorphous silica 73

Figure 4.22 Isosteric heat of adsorption of water on zeolite of CHA and AFI framework type . 75 Figure 5.1 Schematic diagram of CVVP apparatus for measuring $\mathrm{CO}_{2}$ adsorption of activated carbons. 80 
Figure 5.2 Schematic diagram of the adsorption/desorption isotherm and kinetics setup. 82

Figure 5.3 Adsorption isotherms diagrams for $\mathrm{CO}_{2}$ uptakes on (a) Maxsorb III (b) ACF-A20 (c) BPL (d) Norit and (e) honeycomb monolith type activated carbons for the temperatures ranging from $303 \mathrm{~K}$ to $373 \mathrm{~K}$ 86

Figure 5.4 (a): Isotherm data for water vapour onto the type 'RD' silica gel; for experimental data points with: $(\boldsymbol{\square}) T=303 \mathrm{~K} ;(\bigcirc) T=308 \mathrm{~K} ;(\mathbf{\Delta}) T=313 \mathrm{~K}$; $(\square) T=323 \mathrm{~K}$; (+) $T=338 \mathrm{~K}$, for computed data points with solid lines for the Tóth's equation. 88

Figure 5.5 Experimentally measured water uptakes on (a) AQSOA-Z01, (b) AQSOA-Z02 and (c) AQSOA-Z05 zeolites under static conditions 91

Figure 6.1 The schematic of adsorption chiller for continuous cooling 97

Figure 6.2 The schematic of adsorption - desorption processes in the adsorption bed employing pressure - temperature - uptakes co-ordinate system. Here the process A indicates the pressurisation in the adsorption bed with the effects of un-adsorbed adsorbate in void volume, B defines the flow out of high pressurised $\mathrm{CO}_{2}$ from the adsorption cell, the process $\mathrm{C}$ occurs due to the decrease of pressure in the adsorption bed and D indicates low pressure adsorbate flow

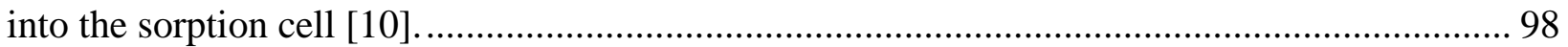

Figure 6.3 P-h diagram of ACCD cycle. Here "o" indicates our experimental data of type RD silica gel-water system. 102

Figure 6.4 COP as a function of total micropore volume ranged from 0.3 to $0.6 \mathrm{cc} / \mathrm{g}$ and pore size for the regeneration temperature of $353 \mathrm{~K}$. Here cc indicates $\mathrm{cm}^{3}$. 104

Figure 6.5 A plot of COP versus desorption temperature for various types of silica gels. 105

Figure 6.6 Influences of pore widths on water production for the evaporator temperature of 280 K. Pore volume is ranged from 0.2 to $0.7 \mathrm{cc} / \mathrm{g}$ and here $\mathrm{cc}$ indicates $\mathrm{cm}^{3}$ 105 
Figure 6.7 A plot of SWP against pore width for various evaporator temperatures. Here the evaporator temperatures range from $274 \mathrm{~K}$ to $303 \mathrm{~K}$ and the regeneration temperature is $353 \mathrm{~K}$. 106

Figure 6.8 Overall performance ratio as a function of pore width for various pore volumes of silica gel. Pore volume is ranged from 0.2 to $0.7 \mathrm{cc} / \mathrm{g}$. 107

Figure 6.9 The plot of isosteric heat of adsorption as a function of pore width for the adsorption of $\mathrm{CO}_{2}$ on carbonaceous material

Figure 6.10 COP against pore widths for (a) pitch based activated carbon (Maxsorb III), (b) activated carbon fibre (ACF-A20), (c) BPL activated carbon and (d) monolith activated carbon based adsorption cooling cycle for the heat source temperatures ranging from $60^{\circ} \mathrm{C}$ to $110^{\circ} \mathrm{C}$ at the cooling load and the heat sink temperatures of $10^{\circ} \mathrm{C}$ and $30^{\circ} \mathrm{C}$, respectively. 111 Figure 6.11 The COP of ADC as a function of pore widths at various cooling load temperatures for the adsorption of $\mathrm{CO}_{2}$ on (a) Maxsorb III, (b) ACF (A-20), (c) BPL activated carbon and (d) monolith activated carbon. Here the driving heat source and the heat sink temperatures are $90{ }^{\circ} \mathrm{C}$ and $10{ }^{\circ} \mathrm{C}$, respectively. 


\section{List of Tables}

Table 2.1 Ideal isotherms for monolayer adsorption................................................... 10

Table 2.2 Empirical isotherms for monolayer adsorption ............................................. 11

Table 2.3 Summary of isosteric heat of adsorption for various adsorbent - adsorbate pairs....... 13

Table 2.4 Summary of Adsorption Cooling Systems..................................................... 18

Table 2.5 Summary of Adsorption Desalination Systems ............................................... 19

Table 3.1 Average bound coherent scattering lengths and atomic form factor for $\mathrm{Si}$, and $\mathrm{O} \ldots . . .33$

Table 3.2 Minimum atom-atom distance used in the RMC simulation .................................. 34

Table 3.3 Well depth potentials and collision diameters of simulated pairs ........................... 46

Table 4.1 Simulation parameters for calculating Lennard-Jones potential ............................. 50

Table 4.2 Simulation parameters for calculating electrostatic potential ................................ 51

Table 5.1 Adsorption isotherm parameters for various carbonaceous materials and $\mathrm{CO}_{2}$ system85

Table 5.2 Correlation coefficients for the two grades of Fuji Davison silica gel + water systems

(The error quoted refers to the $95 \%$ confidence interval of the least square regression of the

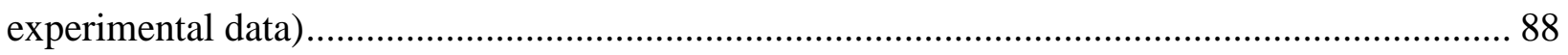

Table 5.3 Parameters of isotherm equations for various AQSOA zeolites - water systems ....... 95

Table 6.1 Parameters of activated carbon and $\mathrm{CO}_{2}$ adsorbate molecules for simulation purposes

Table 6.2 The optimum COP of activated carbons $+\mathrm{CO}_{2}$ assisted adsorption cooling cycle for various heat source temperatures at the cooling load and the heat sink temperatures of $10{ }^{\circ} \mathrm{C}$ and $30{ }^{\circ} \mathrm{C}$ 113 


\section{Nomenclature}

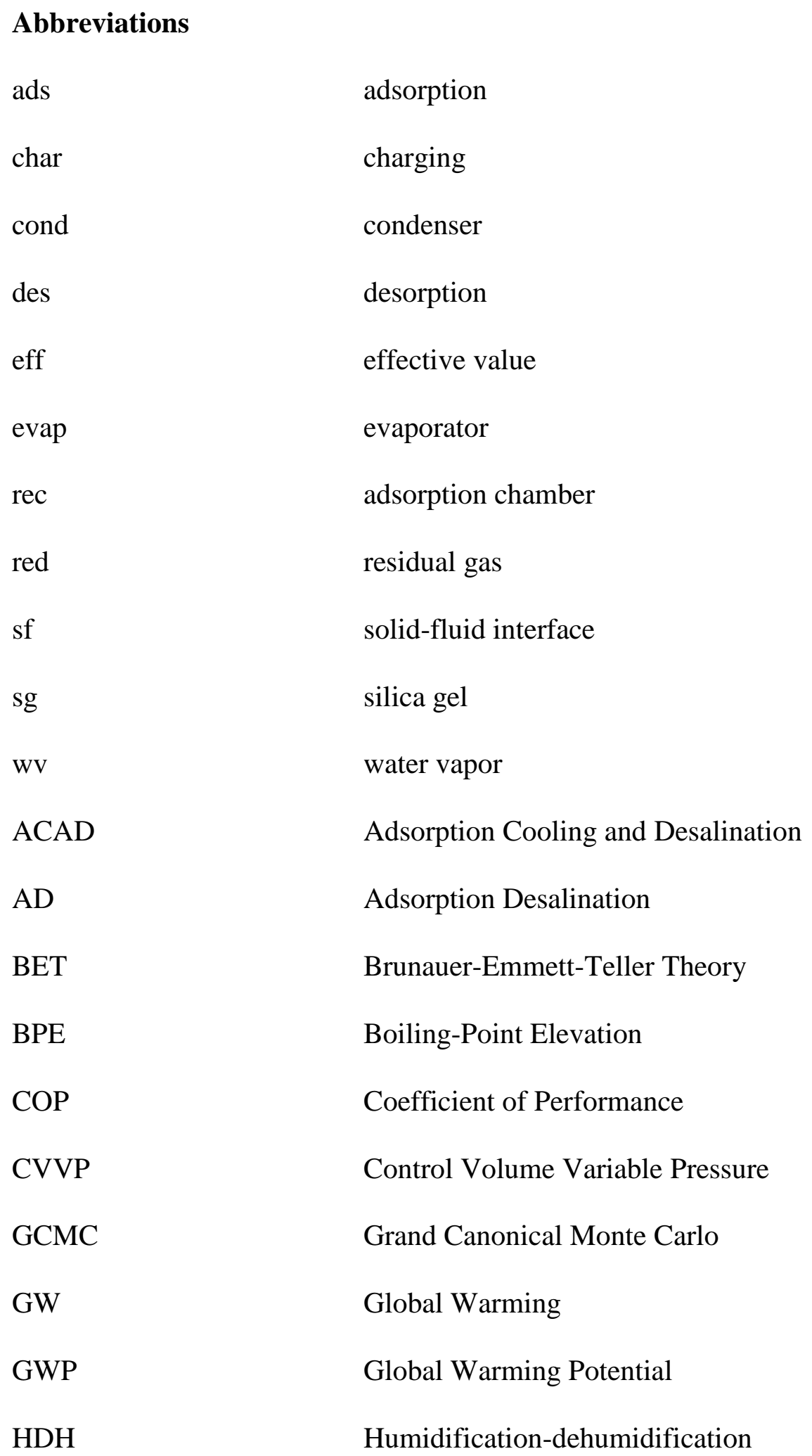


HVAC

IUPAC

MOF

NIST

PID

PR

REFPROP -

RMC

SDWP

XRD

\section{Symbols}

b

c

$c_{p, s}$

$E_{0}$

$E_{a}$

$h$

$h_{f}$

$k$

$k_{a}$

$k_{B}$

$K_{F}$

$K_{L F}$
Heating Ventilation and Air Conditioning

International Union of Pure and Applied Chemistry

Metal Organic Framework

National Institute of Standards and Technology, USA

proportional-integral-derivative

Performance Ratio

Reference Fluid Thermodynamic and Transport Properties

Reverse Monte Carlo

Specific Daily Water Production

X-ray Diffraction

affinity constant

constant in adsorption isotherms

specific heat of sea water

$\mathrm{J} /(\mathrm{kg} \cdot \mathrm{K})$

characteristic energy

$\mathrm{kJ} / \mathrm{mol}$

activation energy of adsorption

$\mathrm{kJ} / \mathrm{mol}$

heat transfer coefficient

$\mathrm{W} /\left(\mathrm{m}^{2} \cdot \mathrm{K}\right)$

enthalpy of sea water

$\mathrm{kJ} / \mathrm{mol}$

thermal conductivity

$\mathrm{W} /(\mathrm{m} \cdot \mathrm{K})$

adsorption rate constant

Boltzmann constant

$\mathrm{J} / \mathrm{K}$

constant for Freundlich adsorption isotherm

constant for Sips adsorption isotherm 


\begin{tabular}{|c|c|c|}
\hline$K_{o}$ & pre-exponential coefficient & - \\
\hline$K_{T}$ & constant for Toth adsorption isotherm & - \\
\hline$\dot{m}$ & mass flow rate & $\mathrm{kg} / \mathrm{s}$ \\
\hline$m_{\text {char }}$ & mass of residue gas in charging chamber & $\mathrm{kg}$ \\
\hline$m_{e q}$ & equilibrium mass & $\mathrm{kg}$ \\
\hline$m_{\text {init }}$ & initial mass & $\mathrm{kg}$ \\
\hline$m_{L F}$ & heterogeneity parameter & - \\
\hline$m_{r e c}$ & mass of initial residue gas in adsorption chamber & $\mathrm{kg}$ \\
\hline$m_{\text {red }}$ & mass of final residue gas in adsorption chamber & $\mathrm{kg}$ \\
\hline$M_{S}$ & mass of adsorbent & $\mathrm{kg}$ \\
\hline$m_{S G}$ & mass of silica gel & $\mathrm{kg}$ \\
\hline$M_{t}$ & total mass & $\mathrm{kg}$ \\
\hline $1 / n$ & heterogeneity parameter & - \\
\hline$n_{1}$ & adsorbate-adsorbent interaction factor & - \\
\hline$P$ & pressure & $\mathrm{Pa}$ \\
\hline$P_{a d s}$ & adsorption pressure & $\mathrm{Pa}$ \\
\hline$P_{\text {dos }}$ & dosing pressure & $\mathrm{Pa}$ \\
\hline$P_{S}$ & saturation pressure & $\mathrm{Pa}$ \\
\hline$q_{a d s}$ & uptake during adsorption & $\mathrm{kg} / \mathrm{kg}$ \\
\hline$q_{\text {des }}$ & uptake during desorption & $\mathrm{kg} / \mathrm{kg}$ \\
\hline$q^{o}$ & the limiting uptake & $\mathrm{kg} / \mathrm{kg}$ \\
\hline$q_{s t}^{o}$ & Isosteric heat of adsorption at zero coverage & $\mathrm{eV}, \mathrm{kJ} / \mathrm{mol}, \mathrm{kJ} / \mathrm{kg}$ \\
\hline$q_{t}$ & uptake at time $t$ & $\mathrm{~kg} / \mathrm{kg}$ \\
\hline
\end{tabular}




\begin{tabular}{|c|c|c|}
\hline$w$ & interaction energy between adsorbed molecules & $\mathrm{kJ} / \mathrm{mol}$ \\
\hline$R$ & ideal gas constant & $\mathrm{J} /(\mathrm{mol} \cdot \mathrm{K})$ \\
\hline$t$ & constant for Toth adsorption isotherm & - \\
\hline$t_{1}$ & surface heterogeneity & - \\
\hline$T$ & temperature & $\mathrm{K}$ \\
\hline$U$ & potential & $\mathbf{J}$ \\
\hline$U_{L J}$ & Lenard-Jones potential & $\mathrm{J}$ \\
\hline$U_{E}$ & electrostatic potential & $\mathrm{J}$ \\
\hline$U_{I}$ & induction potential & $\mathrm{J}$ \\
\hline$U_{m M}$ & total interaction potential & $\mathrm{J}$ \\
\hline$v_{\text {ext }}$ & total external potential & $\mathrm{meV}$ \\
\hline$v_{L J}$ & Lennard -Jones potential & $\mathrm{meV}$ \\
\hline$v_{E}$ & Electrostatic potential & $\mathrm{meV}$ \\
\hline$v_{I}$ & Induction potential & meV \\
\hline$V_{\text {char }}$ & volume of charging chamber & $\mathrm{m}^{3}$ \\
\hline$V_{\text {rec }}$ & volume of adsorption chamber & $\mathrm{m}^{3}$ \\
\hline$V_{\text {rec }}$ & volume of adsorption chamber & $\mathrm{m}^{3}$ \\
\hline$z$ & the distance between adsorbent - adsorbate & $\AA$ \\
\hline$z_{o}$ & the distance for maximum $q_{s t}^{o}$ & $\AA$ \\
\hline$\beta$ & loading factor & - \\
\hline$\varepsilon_{0}$ & vacuum permittivity & $\mathrm{C} /(\mathrm{V} \cdot \mathrm{m})$ \\
\hline$\varepsilon$ & potential & $\mathrm{J}$ \\
\hline$\varepsilon_{i j}$ & pair well depth potential & $\mathbf{J}$ \\
\hline
\end{tabular}




\begin{tabular}{|c|c|c|}
\hline$\theta$ & surface adsorbate coverage & - \\
\hline$\mu$ & chemical potential & $\mathrm{J} / \mathrm{mol}$ \\
\hline$\rho_{\text {char }}$ & density of gas during charging & $\mathrm{kg} / \mathrm{m}^{3}$ \\
\hline$\rho_{\text {rec }}$ & density of residue gas & $\mathrm{kg} / \mathrm{m}^{3}$ \\
\hline$\sigma$ & collision diameter & $\mathrm{m}$ \\
\hline$\sigma_{i j}$ & pair collision diameter & $\mathrm{m}$ \\
\hline$\gamma_{A}$ & coefficient of Lenard-Jones potential & - \\
\hline$\gamma_{R}$ & coefficient of $\mathrm{LJ}$ potential & - \\
\hline$\phi_{\text {dipole }}$ & electrical potential of a dipole & $\mathrm{V}$ \\
\hline$\phi_{m}$ & the minimum potential energy & $\mathrm{J}$ \\
\hline$\phi_{\text {quadrupole }}$ & electrical potential of a quadrupole & $\mathrm{V}$ \\
\hline $\mathbf{E}$ & electric field vector & $\mathrm{V} / \mathrm{m}$ \\
\hline $\mathbf{E}_{\text {dipole }}$ & electric field vector of a dipole & $\mathrm{V} / \mathrm{m}$ \\
\hline $\mathbf{E}_{\text {quadrupole }}$ & electric field vector of a quadrupole & $\mathrm{V} / \mathrm{m}$ \\
\hline $\mathbf{F}$ & electric field vector & $\mathrm{V} / \mathrm{m}$ \\
\hline $\mathbf{I}$ & $3 \times 3$ unit diagonal matrix & - \\
\hline $\mathbf{r}$ & position vector between two multipoles & $\mathrm{m}$ \\
\hline $\mathbf{r}^{\mathrm{T}}$ & transposed position vector & $\mathrm{m}$ \\
\hline $\mathbf{r}_{\mathbf{i}}$ & position vector of the $i$ th atom of the adsorbate molecule & $\mathrm{m}$ \\
\hline $\mathbf{r}_{\mathbf{j}}$ & position vector of the $j$ th carbon atom & $\mathrm{m}$ \\
\hline \multirow[t]{2}{*}{$\mathbf{r}_{\mathrm{ij}}$} & position vector from the $i$ th carbon to the $j$ th atom of the & \\
\hline & adsorbate molecule & $\mathrm{m}$ \\
\hline$\alpha$ & polarisability matrix & $\AA^{3}$ \\
\hline
\end{tabular}


$\Theta$

$\boldsymbol{\mu}$

$\boldsymbol{\mu}^{\mathrm{I}}$

$\boldsymbol{\mu}_{\mathrm{H}_{2} \mathrm{O}}^{\mathrm{I}}$

$\boldsymbol{\mu}_{\mathrm{C}}^{\mathrm{I}}$ quadrupole moment matrix

DÅ

dipole moment vector

induced dipole moment vector

induced dipole moment vector of water molecule

induced dipole moment vector of carbon atom
D

D

D

$\mathrm{D}$

D 


\section{Table of Contents}

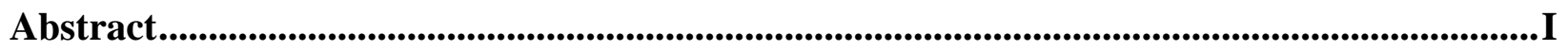

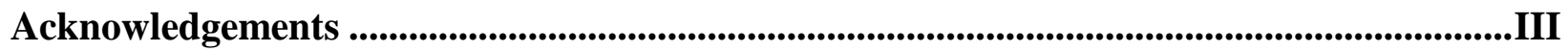

Publication Arising from This Thesis ................................................................................. IV

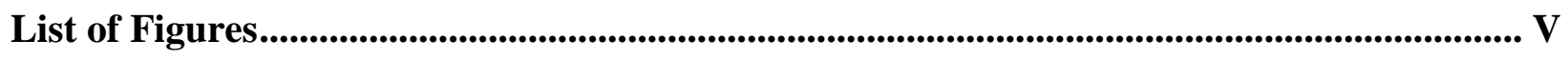

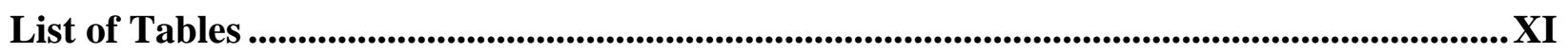

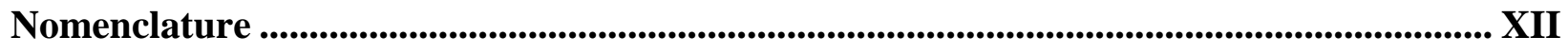

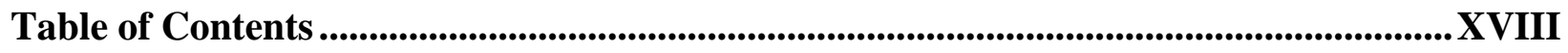

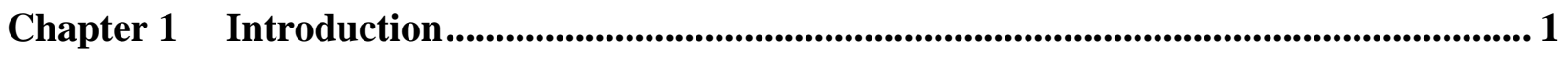

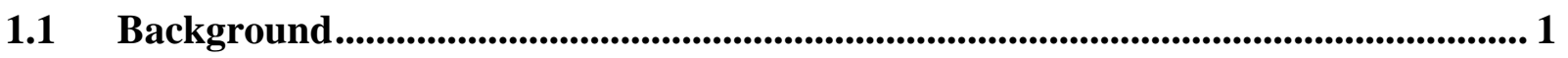

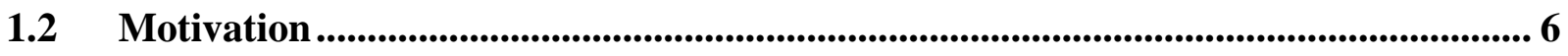

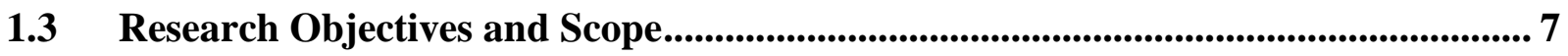

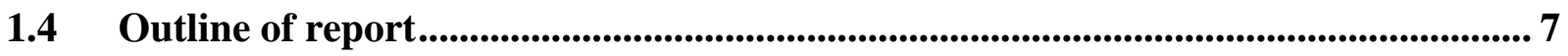

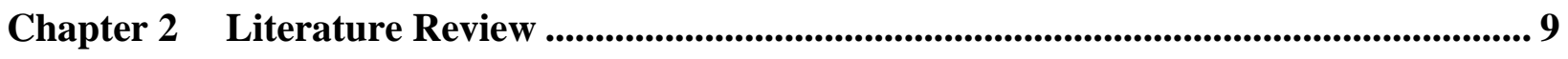

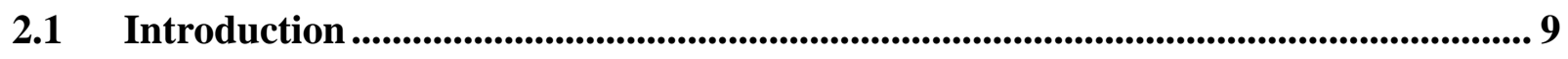

2.2 Adsorption Isotherms and Kinetics .............................................................................9

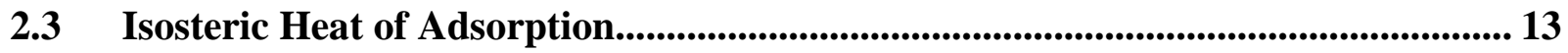

2.4 Adsorption Assisted Cooling and Desalination ..................................................... 14

2.4.1 Adsorption Cooling ............................................................................................... 17

2.4.2 Adsorption Desalination ....................................................................................... 19 
Chapter 3 Modelling and Simulation of Isosteric Heat of Adsorption at Zero Coverage 22

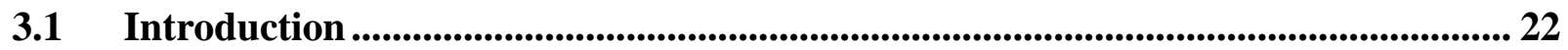

3.2 Previous Studies on Adsorbent Structures and Modellings ................................. 23

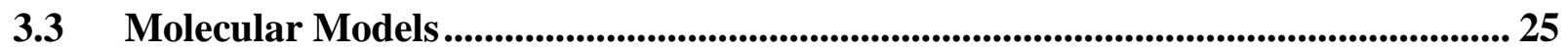

3.3.1 Molecular Model of Lattice Surface............................................................... 26

Interaction Potential of Lattice Surface and Adsorbate Molecules.................................. 27

3.3.2 Molecular Model of Amorphous $\mathrm{SiO}_{2}$ Surface and Water Molecules............... 31

Interaction Potential of Amorphous $\mathrm{SiO}_{2}$ Surface and Adsorbate Molecules .................... 40

3.3.3 Molecular Model of Framework Structure and Water Molecules................... 42

Interaction Potential of Framework Structure and Adsorbate Molecules ......................... 44

3.4 Isosteric Heat of Adsorption at Zero Coverage ......................................................... 46

3.4.1 Isosteric Heat of Adsorption for Parallel Slit Pore ......................................... 47

3.4.2 Isosteric Heat of Adsorption for Cylindrical Pore............................................ 48

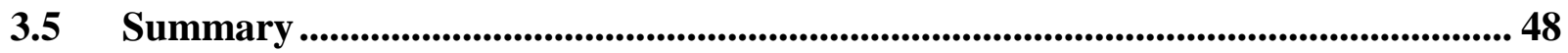

Chapter 4 Simulation Results of Isosteric Heat of Adsorption at Zero Coverage ............. 50

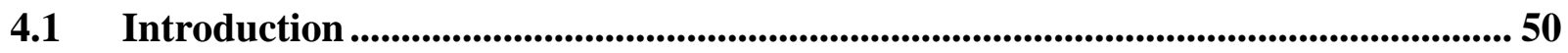

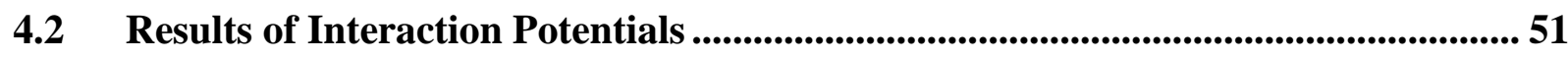

4.2.1 Interaction Potential of Graphite with Polar Molecules ..................................... 51

4.2.2 Interaction Potential of Silica with Water Molecule ......................................... 58 
4.2.3 Interaction Potential of Zeolites with Water Molecule 65

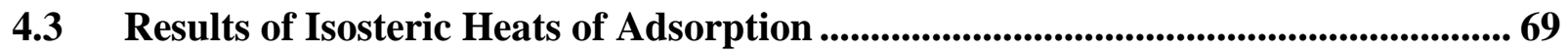

4.3.1 Isosteric Heats of Adsorption of Graphite with Water, Methanol and Ethanol

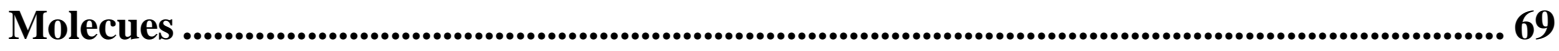

4.3.2 Isosteric Heats of Adsorption of Silica with Water Molecule ............................ 73

4.3.3 Isosteric Heats of Adsorption at Zero Coverage for Zeolites with Water

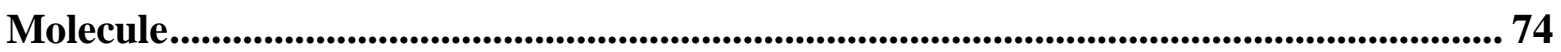

4.4 Summary of Chapter 4 ............................................................................................ 76

4.4.1 Graphite and Water, Methanol and Ethanol Molecules ..................................... 76

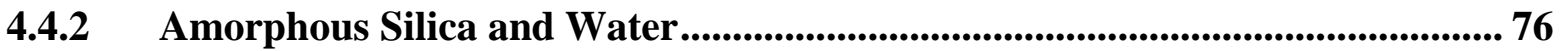

4.4.3 Zeolite and Water .................................................................................... 77

Chapter 5 Measurement of Adsorption Uptakes under Static Conditions......................... 79

$5.1 \quad$ Experimental Investigation ............................................................................................... 79

5.1.1 Measurement of $\mathrm{CO}_{2}$ Uptakes on Activated Carbon ............................................. 79

5.1.2 Measurement of Water Uptakes on Silica Gels and AQSOA Zeolites............. 81

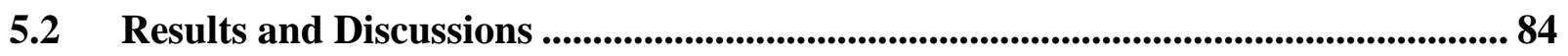

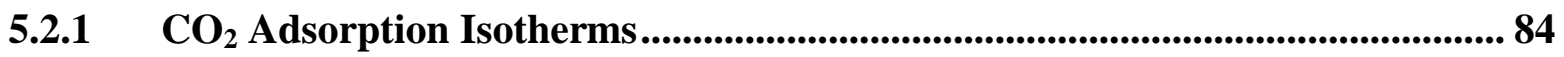

5.2.2 Water Adsorption Isotherms .................................................................. 87

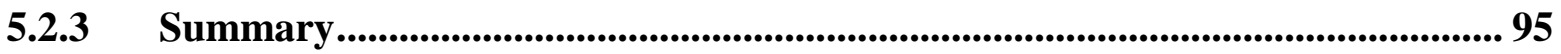


Chapter 6 Thermodynamic Frameworks for Adsorption Cooling and Desalination Systems 96

6.1 Thermodynamic Framework of Adsorption Cooling and Desalination 96

6.2 P-h (Pressure-Enthalpy) Diagram of Adsorption Assisted Cooling and

Desalination. 101

6.3 Performances of Silica Gel - Water based Cooling and Desalination. 103

6.4 Performances of Activated Carbons - $\mathrm{CO}_{2}$ based Cooling and Refrigeration..... 107

6.5 Summary 114

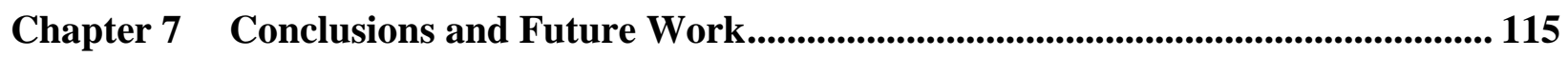

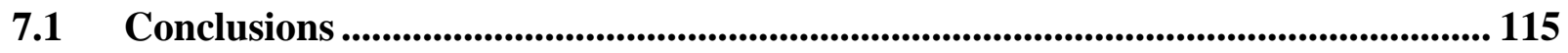

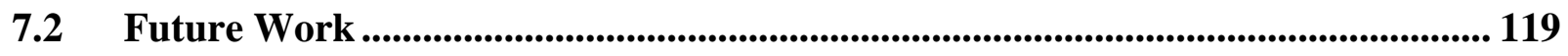

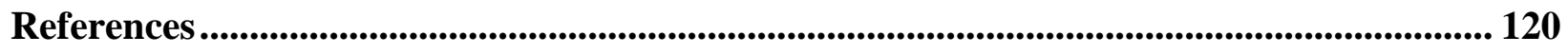

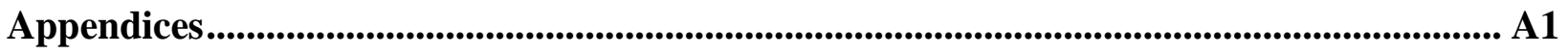

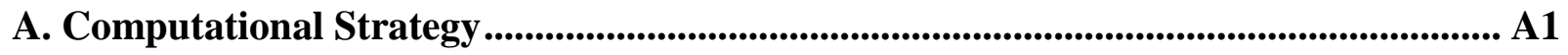

A1. Multipole Expansion of Energy .................................................................................. A1

A2. Reduced Multipole Expansion of Energy ............................................................... A1

A3. Electric Field and Energy of multipole .................................................................... A2

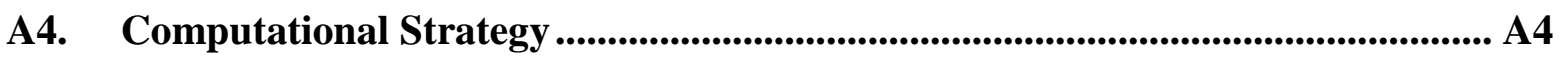

A5. Dipole-quadrupole Interaction Potential...................................................... A7

A6. Interaction Potential of Water on Graphite .................................................... A9 
A7. Interaction Potential of Methanol on Graphite

A8. Interaction Potential of Ethanol on Graphite ...................................................... A12

A9. Coordinate Transformation............................................................................... A14 


\section{Chapter 1 Introduction}

\subsection{Background}

Adsorption is the adhesion of particles (atoms, ions, molecules as adsorbate) to a porous surface of adsorbent while desorption is correspondingly a the reversible process [1]. The adsorptiondesorption phenomena are generally employed for thermal compression processes for low temperature heat transmission applications such as adsorption cooling, desalination and dehumidification [2-4].

In an adsorption process as shown schematically in Figure 1.1, the motions of adsorbate molecules are restrained by binding forces between them in the pore surfaces, and the energy is

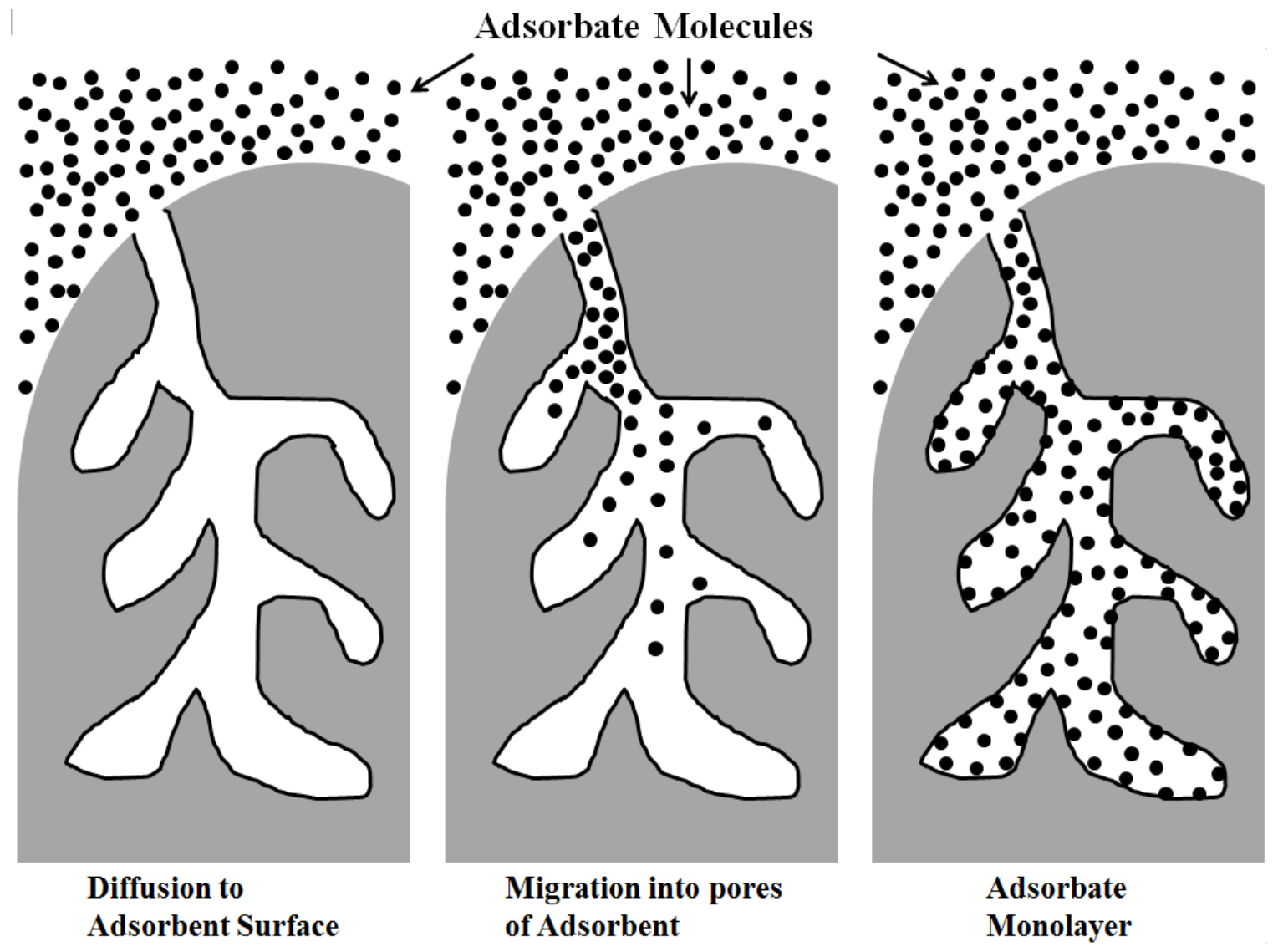

Figure 1.1 The schematic adsorption process 
released to the environment due to the exothermic process [5]. On the other hand, due to the addition of heat or pressure swing, the trapped molecules in the pores escape in an endothermic process.

When the molecular motion of the adsorbate balances in the pores and surfaces of an adsorbent with respect to all directions of the adsorption pressure, adsorption equilibrium is achieved. Therefore, the lower temperature shifts the balance towards the adsorption process while the higher temperature accelerates the desorption process. Thus, the reversible adsorption-desorption process advocates a cyclic adhesion and release of the adsorbate from the porous solid adsorbent, and can be applied for the generation of low temperature heat transformation processes, i.e., cooling cycle. The low temperature occurs in the evaporator by amalgamation of adsorptiontriggered-evaporation and desorption-activated-condensation [6].

The amount of adsorbate on a porous adsorbent under equilibrium conditions can be represented in a pressure-uptake (P-q) coordinate system at a constant temperature (T), which is known as an adsorption isotherm. The isotherm curves are essential for understanding the Henry and saturated regions of an adsorbent-adsorbate system. Practically, isotherms are presented through a collected set of experimentally measured data. The adsorption isotherm data are used to calculate the differential amount of refrigerant exchanged in each adsorption/desorption cycle for the design of adsorption assisted cooling, desalination, purification and gas storage systems. The isotherm classifications are based on the shape of adsorption isotherm curves ranging from Henry's region to the saturated pressure. At first, adsorption isotherms are classified into five BET types according to IUPAC classification as shown in Figure 1.2 [5, 7], where the $\mathrm{x}$ axis is the relative pressure $\left(\mathrm{P} / \mathrm{P}_{\mathrm{s}}, \mathrm{P}\right.$ and $\mathrm{P}_{\mathrm{s}}$ are adsorption and saturation pressure in unit $\left.\mathrm{Pa}\right)$ and the $\mathrm{y}$ axis is uptake (in unit $\mathrm{g} / \mathrm{g}$ ). 


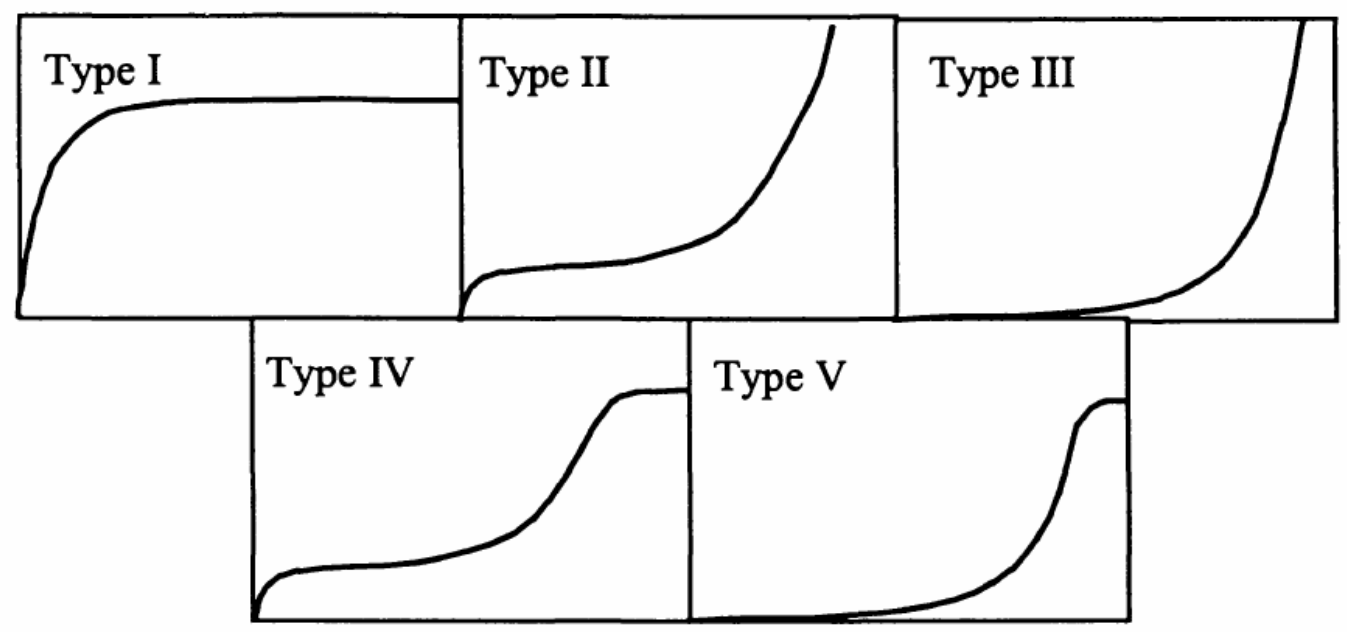

Figure 1.2 IUPAC Classifications of Adsorption Isotherms

Type I describes an adsorption process that mainly occurrs in the microporous adsorbent with maximum loading under equilibrium conditions. Type II characterises the nature of adsorption uptake on a macroporous adsorbent with strong adsorbate-adsorbent interactions at relatively higher pressure. On the other hand, type III provides weaker interactions at the beginning of adsorption. For type IV isotherm, monolayer adsorption with capillary condensation is observed, and type V shows multilayer adsorption [8].

It should be noted that the adsorption kinetics quantitatively describes the dynamic behaviours of adsorbate uptakes on porous adsorbent for various temperatures and pressures. Generally, the porous solid with large surface area and micro-pore volume yields high uptakes while those with large pore networks are responsible for faster kinetics [5].

During an adsorption process, the adsorbate molecules are held near to the pores of the adsorbent surface and pores by van der Waals force fields, and the amount of adsorbed heat, mass of adsorbate and gaseous molecules and chemical potentials of adsorbed $\left(\mu_{a}\right)$ and the gaseous phases $\left(\mu_{g}\right)$ are necessary for fully describing the thermodynamic state of adsorbent - adsorbate systems. Adsorption isosteric heat $\left(\mathrm{q}_{\mathrm{st}}\right)$ is defined as the difference between the energies 
processed by the adsorbate molecules in both gaseous and adsorbed phases, as illustrated schematically in Figure 1.3 [9].

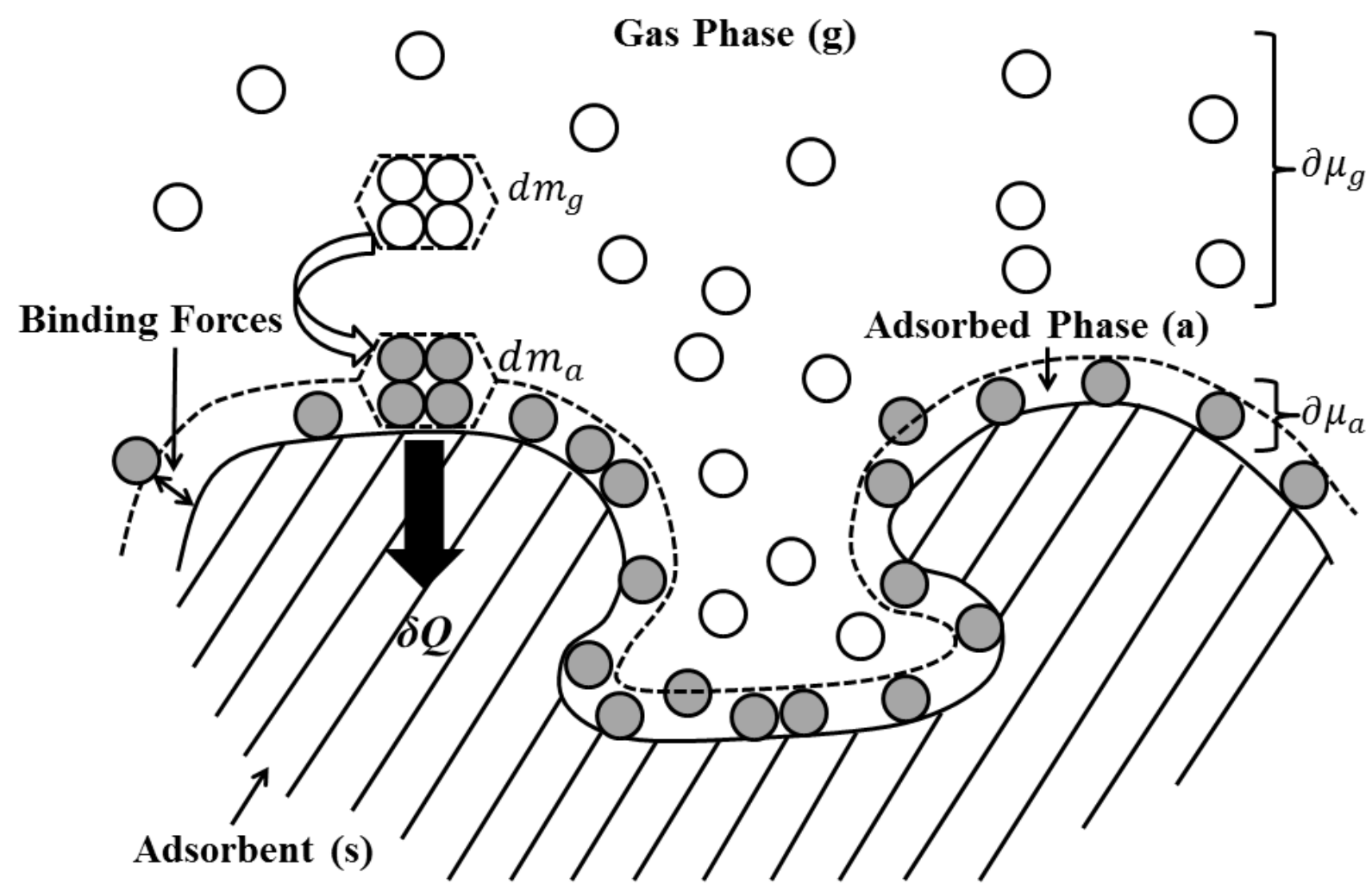

Figure 1.3 The schematic formalism of adsorption isosteric heat

The knowledge of isosteric heat at zero coverage $\left(q_{s t}{ }^{o}\right)$ is necessary for calculating the energetic performances of adsorption assisted cooling, desalination and gas storage [10-12]. At zero surface coverage, the density of adsorbate molecules in the adsorbent pores and surface is relatively very low and the pressure is considered close to zero (not zero numerically, $\mathrm{P} \rightarrow 0$ ), which allows the adsorbate-adsorbate interactions to be negligible. It should also be noted here that the modelling of $\left(q_{s t}{ }^{o}\right)$. at higher pressure is complicated due to the presence of intermolecular interactions along the adsorbates. The modelling of $q_{s t}^{o}$ has been studied by molecular simulations for many commonly applied adsorbent and adsorbate pairs $[13,14]$. 
A good number of adsorption isotherm models are proposed on the basis of either the mechanism of adsorption processes or the curve-fitting results that are best matched with experimental data $[5,15]$. Both first and second order adsorption kinetics are also used for the analysis of adsorption experimental data, modelling and simulation $[11,16,17]$. These adsorption isotherm and kinetics results are made to be conveniently ready-to-use by the simulation of the isosteric heat of adsorption. However, there has been a lack of theoretical explanation for the formalism of the isosteric heat of adsorption. Therefore, a thermodynamic study of isosteric heat at zero coverage would be essential from a theoretical point of view.

In the arena of adsorption cooling and desalination systems, the basic characteristics such as adsorption isotherms, kinetics and isosteric heats are commonly used to quantitatively describe their thermodynamic performances employing the mass and energy balances $[10,11,18]$.

The adsorption assisted cooling and desalination systems are scalable, nearly free of moving parts, environmentally friendly and driven by the low grade waste heat (temperatures are ranged from $55{ }^{\circ} \mathrm{C}$ to $90{ }^{\circ} \mathrm{C}$ ). The conventional vapour compression cooling system utilises non-natural refrigerants (such as R132a, R507a, R410, R1234yf etc.) [19]. On the other hand, the thermallydriven adsorption cooling employs natural refrigerants (e.g., water, ammonia), and can be conveniently integrated with the currently used cooling cum desalination systems where the waste heat source from a co-generation system (such as power plant [20], internal combustion engines [21, 22], solar systems [23, 24], etc) are used without extra energy consumption. Secondly, the adsorption device employs solid adsorbents for the design of thermal compressor or sorption bed where the refrigerant (water vapour) is adsorbed and desorbed to transfer water vapour from evaporator to bed and from bed to the compressor per adsorption-desorption cycle. The other heat driven cooling system such as absorption chiller utilises liquid desiccant-solution 
for producing cooling by absorption-triggered-evaporation and solution regeneration-activatedcondensation [25]. The conventional absorption chiller requires very high regeneration temperature $\left(>100{ }^{\circ} \mathrm{C}\right.$ ) and $\mathrm{LiBr}$ or $\mathrm{LiCl}$ solutions are toxic. It should be noted here that the adsorption desalination systems as an emerging thermally-driven method are proved to be energy efficient and environmentally friendly due to its ability to produce high-grade potable water and cooling effects with only a single heat source input of $80^{\circ} \mathrm{C}$ or lower [26].

\subsection{Motivation}

In this thesis, the theoretical insight of isosteric heat of adsorption at zero coverage will be focused.

Since a good number of experimentally measured isosteric heat of adsorption data for adsorption of graphite and polar molecules, activated carbon and carbon dioxide, silica gel and water, zeolites (AQSOA-Z01, Z02 and Z05) and water systems are available in literature, a theoretical

analysis for predicting the isosteric heat of adsorption at zero coverage $\left(q_{s t}^{o}\right)$ has to be developed. The isosteric heat of adsorption at zero coverage is closely related to the structure of adsorbent materials. The adsorbent structure analysis is also simulated theoretically to understand its pore size and volume. The combination of adsorbent structure and the interaction potential between adsorbent and adsorbate are necessary to calculate the isosteric heat of adsorption and also to predict the energetic performance of adsorption cooling and desalination systems.

The value of isosteric heat of adsorption at zero coverage can significantly influence the performances of adsorption assisted cooling and desalination cycle as a function of pore width of adsorbent materials. Therefore, the knowledge of isosteric heat of adsorption is needed to design the adsorption bed. 


\subsection{Research Objectives and Scope}

This thesis aspires to develop a theoretical foundation to calculate the isosteric heat of adsorption at zero coverage for various adsorbent and adsorbate systems. Employing the theoretical formulation of isosteric heat, the energetic performances of adsorption cooling and desalination systems are predicted in terms of the pore width and volume of adsorbent materials. A connection has been established between adsorbent structures and the adsorption energy from a thermodynamic perspective.

These objectives are achieved by including the following research activities:

1. To construct structure models of adsorbents and adsorbates based on crystallography knowledge of various types of interfaces including lattice, amorphous surface and frameworks.

2. To investigate various interaction potentials such as Lennard-Jones, electrostatic and induction between adsorbate molecules with the constructed adsorbent structures.

3. To develop the thermodynamic formalism of isosteric heat as a function of pore width.

4. To investigate experimentally the amount of water vapour and $\mathrm{CO}_{2}$ uptakes on silica gel, zeolites and activated carbons for the temperatures ranging from 25 to $65{ }^{\circ} \mathrm{C}$ and pressures up to $10 \mathrm{kPa}$ under both static and dynamic conditions.

5. To conduct a numerical analysis for calculating the performances of adsorption cooling and desalination systems in terms of $\mathrm{COP}$ and specific daily water production employing the proposed formulations and experimentally measured isotherm data.

\subsection{Outline of report}

This thesis starts with a brief introduction about the background, motivations and objectives to be achieved from the proposed research. These are illustrated in Chapter 1. 
Chapter 2 reviews the literature and introduces the state of the arts of research works related to the topics, including various types of cooling technologies, desalination technologies, the adsorption phenomenon, isotherms and kinetics, the principle of adsorption cooling-desalination cycles and the modelling and simulative researches of adsorption chiller and desalination. An integrated technical route is proposed to achieve all the research objectives based on the literature review.

Chapter 3 focuses on the modelling and simulation of isosteric heat of adsorption at zero coverage for various adsorbent + adsorbate systems. In this chapter, the molecular models of adsorbate materials and adsorbent are constructed, and the interactions between the adsorbent molecules with the constructed molecular models are studied. The values of isosteric heat for these pairs are then developed based on the calculation of interaction potential and pore model.

Chapter 4 presents the simulation results of the potential well and isosteric heats as a function of pore width for various orientations of adsorbate molecules, which is new.

Chapter 5 describes the detailed procedures of experimental study for the measurement of adsorption isotherm and kinetics for various adsorbent/adsorbate pairs.

Chapter 6 presents the performance evaluations of adsorption cooling and desalination systems in terms of COP and SWP as a function of pore width. These results are compared with experimentally measured data for verification purposes.

Chapter 7 concludes the findings of the current research and the directions of future work to improve the design, modelling and optimisation of adsorption beds for cooling and desalination purposes. 


\section{Chapter 2 Literature Review}

\subsection{Introduction}

Firstly, the phenomenon of adsorption is described in detail from the viewpoints of adsorption isotherm and kinetic models. The explanation related to the theoretical development and understanding on adsorption isotherms and kinetics are provided here.

Secondly, the state of the art of simulation and theoretical formalisms of isosteric heat of adsorption will be given for various adsorbent + adsorbate pairs, which will provide some valuable insights on the constructions of connection between isosteric heat and the structure of porous adsorbents.

Thirdly, some novel cooling technologies being researched, such as $\mathrm{CO}_{2}$ cooling are introduced and their advantages are discussed in order to elucidate the beneficial properties of these novel cooling technologies on concerns of environmental protection and economic efficiency.

Fourthly, a number of adsorption cooling and desalination systems are introduced. A brief description on these technologies is summarised.

\subsection{Adsorption Isotherms and Kinetics}

The adsorption isotherms and kinetics that characterise the equilibrium uptakes and adsorption rates are important properties of adsorption phenomenon in the modelling and simulation of adsorption cycles. During the history of more than a century, a good number of adsorption isotherms and kinetics models were derived and established.

The ideal isotherm models for monolayer adsorption are furnished in Table 2.1[5]. 
Table 2.1 Ideal isotherms for monolayer adsorption

\begin{tabular}{|c|c|l|}
\hline Type & Isotherm & \multicolumn{1}{c|}{ Remarks } \\
\hline Henry & $b P=\theta$ & $\begin{array}{l}\text { Applicable at Henry Region } \\
\text { (low pressure range) }\end{array}$ \\
\hline Langmuir & $b P=\frac{\theta}{1-\theta}$ & $\begin{array}{l}\text { Localized and homogeneous } \\
\text { adsorption is assumed. }\end{array}$ \\
\hline Volmer & $b P=\frac{\theta}{1-\theta} \exp \left(\frac{\theta}{1-\theta}\right)$ & $\begin{array}{l}\text { The mobility of adsorbed } \\
\text { molecules is allowed while } \\
\text { the interaction is not. }\end{array}$ \\
\hline Fowler-Guggenheim & $b P=\frac{\theta}{1-\theta} \exp \left(\frac{-c w \theta}{R T}\right)$ & $\begin{array}{l}\text { The interaction is considered } \\
\text { while mobility is not. }\end{array}$ \\
\hline Hill-deBoer & $b P=\frac{\theta}{1-\theta} \exp \left(\frac{\theta}{1-\theta}\right) \exp (-c \theta)$ & $\begin{array}{l}\text { Both mobility and lateral } \\
\text { interaction among adsorbed } \\
\text { molecules are considered. }\end{array}$ \\
\hline
\end{tabular}

${ }^{*} b$ is affinity constant that measures how strong an adsorbate molecule is attracted onto a surface. $*_{*} \mathrm{c}=\frac{z w}{R T}$, where $z$ is coordination number, $w$ is interaction energy between adsorbed molecules, $R$ is gas constant and $\mathrm{T}$ is the temperature.

The widely applied theoretical multilayer adsorption isotherms include the BET (BrunauerEmmett-Teller) isotherm [27] and it is later extended to finite width, n-number of layers [7, 28]. The MacMillan-Teller (MET) isotherm [29] is an adsorption model similar to the BET isotherm that takes surface tension effects into consideration. Another well-known multilayer adsorption isotherm is the FHH (Frenkel-Halsey-Hill) isotherm [30-32]. The theoretical isotherm that fits multiple types of isotherm according to IUPAC is proposed to overcome limitations of existing isotherm model [33, 34], which also explains the surface energy and surface structural heterogeneity factors. Based on experimental data, empirical adsorption isotherms can be obtained through methods such as curve fitting. Some frequently applied empirical isotherms for monolayer adsorption are given as in Table 2.2 [15]. 
Table 2.2 Empirical isotherms for monolayer adsorption

\begin{tabular}{|c|c|l|}
\hline Type & Isotherm & Remarks \\
\hline Freundlich & $\theta=K_{F} P^{1 / n}$ & $\begin{array}{l}\text { The earliest non-ideal and } \\
\text { reversible adsorption isotherm } \\
\text { not restricted to the formation } \\
\text { of monolayer. }\end{array}$ \\
\hline Dubinin-Radushkevich & $\theta=\exp \left[-\left(\frac{R T \ln \left(P_{S} / P\right)}{\beta E_{0}}\right)^{2}\right.$ & $\begin{array}{l}\text { Successfully fits high solute } \\
\text { and intermediate range of } \\
\text { concentration but not } \\
\text { applicable at Henry region. }\end{array}$ \\
\hline Toth & $\theta=\frac{K_{T} P}{\left[1+\left(K_{T} P\right)^{t}\right]^{1 / t}}$ & $\begin{array}{l}\text { Useful in heterogeneous } \\
\text { adsorption systems with } \\
\text { satisfactory low and high-end } \\
\text { boundary of concentrations. }\end{array}$ \\
\hline $\begin{array}{c}\text { Langmuir- Freundlich } \\
\text { (Sips equation) }\end{array}$ & $\theta=\frac{\left(K_{L F} P\right)^{m_{L F}}}{1+\left(K_{L F} P\right)^{m_{L F}}}$ & $\begin{array}{l}\text { It reduces to Freundlich } \\
\text { isotherm at low pressure area } \\
\text { and Langmuir isotherm at high } \\
\text { preesure area. }\end{array}$ \\
\hline
\end{tabular}

*Exponential items such as $1 / n, m_{L F}$ are the heterogeneity parameter that characterises the width and asymmetry of the distribution $\chi(\varepsilon)[35,36]$.

Although they provide no information about the chemical or physical mechanism of adsorption and the parameters used in the isotherms are only valid for particular experimental conditions, these empirical isotherms that are obtained through curve fitting have high precision in specific pressure range [37]. Therefore, these isotherms that are verified with experimental data have been widely applied in the modelling and simulation of adsorption processes.

The adsorption kinetics defines the variation of uptake of a specific adsorbent + adsorbate pair against time until the adsorption equilibrium is thermodynamically achieved [5]. It characterise the speed of a adsorption process, which can be measured by adsorption experiments. The adsorption kinetics are important as the diffusion of adsorbed particles on adsorbent is applied in catalysis, metallurgy, microelectronics, material science, etc[1]. 
The pseudo first-order kinetic model as proposed by Lagergren is derived based on the assumption that the adsorption rate is proportional to the number of unoccupied adsorptive sites [38], and the equation is given by

$$
\frac{d q_{t}}{d t}=k_{1}\left(q^{o}-\mathrm{q}_{t}\right)
$$

, where $q^{o}$ and $\mathrm{q}_{t}$ are the adsorption capacities at equilibrium and time $t,\left(q^{o}-\mathrm{q}_{t}\right)$ represents to the number of unoccupied adsorptive sites and $k_{1}$ is the constant of pseudo first-order adsorption and it yields

$$
\log \left(q^{o}-\mathrm{q}_{t}\right)=\log \left(q^{o}\right)-\frac{k_{1}}{2.303} t
$$

The second order adsorption is obtained by applying a second order adsorption rate as [39, 40].

$$
\frac{d q_{t}}{d t}=k_{1}\left(q^{o}-\mathrm{q}_{t}\right)^{2}
$$

which also shows

$$
\frac{t}{q_{t}}=\frac{1}{k_{2}\left(q^{o}\right)^{2}}+\frac{1}{q^{o}} t
$$

The relationship between adsorption rate constant $k_{a}$ and temperature $T$ is represented in an Arrhenius form

$$
k_{a}=k_{0} \exp \left(-\frac{E_{a}}{R T}\right)
$$

where $k_{0}$ is the temperature independent factor, $E_{a}$ is activation energy of adsorption, $R$ is the ideal gas constant and $\mathrm{T}$ is absolute temperature $[16,41]$.

Both first and second order adsorption kinetics have been used in the analysis of adsorption experimental data, modelling and simulations $[11,16,17]$. 


\subsection{Isosteric Heat of Adsorption}

The isosteric heat of adsorption describes the energetic performances of an adsorption process in a pressure-temperature-uptake coordinate system employing the Clausius - Clapeyron equation. Up to now, a thermodynamic framework describing the isosteric heat of adsorption with respect to adsorbent structure and adsorbate orientation is not well established. It should be noted here that the basic concept of calculating the isosteric heats employing the Grand Canonical Monte Carlo simulation is well established. A summary of the simulation techniques for the isosteric heat of adsorption is furnished in Table 2.3

Table 2.3 Summary of isosteric heat of adsorption for various adsorbent - adsorbate pairs

\begin{tabular}{|c|c|c|c|}
\hline $\begin{array}{l}\text { Adsorbent+adsorbate } \\
\text { pair }\end{array}$ & $\begin{array}{l}\text { Adsorbent } \\
\text { Structure }\end{array}$ & $\begin{array}{c}\text { Interaction } \\
\text { Potential }\end{array}$ & Remarks \\
\hline Silica gel + water [6] & $\begin{array}{l}\text { Layers of silicon } \\
\text { and oxygen atoms + } \\
\text { parallel slit-shaped } \\
\text { pore model }\end{array}$ & $\begin{array}{l}\text { Steele's } 10-4-3 \\
\text { Lennard-Jones } \\
\text { interaction } \\
\text { potential }\end{array}$ & $\begin{array}{l}\text { Clausius-Clapeyron equation } \\
\text { is applied to Henry's region }\end{array}$ \\
\hline $\begin{array}{l}\text { Carbon }+\mathrm{N}_{2}, \mathrm{Ar}, \mathrm{CO}_{2}, \\
\mathrm{CH}_{4}, \mathrm{He}, \mathrm{H}_{2}[13]\end{array}$ & $\begin{array}{lr}\text { Layers of } & \text { graphite } \\
\text { lattice }+ & \text { parallel } \\
\text { slit-shaped } & \text { pore } \\
\text { model } & \end{array}$ & $\begin{array}{l}\text { Steele's } 10-4-3 \\
\text { Lennard-Jones } \\
\text { interaction } \\
\text { potential }\end{array}$ & $\begin{array}{l}\text { Clausius-Clapeyron equation } \\
\text { is applied to Henry's region }\end{array}$ \\
\hline $\begin{array}{l}\text { Graphite }+\mathrm{CO}, \mathrm{HCN}, \\
\mathrm{H}_{2} \mathrm{C} \mathrm{O} \text { and } \mathrm{H}_{2} \mathrm{O} \text { [42] }\end{array}$ & $\begin{array}{l}\text { Layers of graphite } \\
\text { lattice }\end{array}$ & $\begin{array}{l}\text { Energy } \\
\text { expansion } \\
\text { formulation }\end{array}$ & $\begin{array}{l}\text { Approximate energy required } \\
\text { to keep the molecule with } \\
\text { thermal energy }\left(\frac{n}{2}+1\right) k T \text { at } \\
\text { its equilibrium configuration } \\
\text { on the surface of the substrate }\end{array}$ \\
\hline 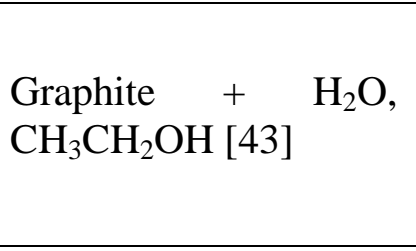 & $\begin{array}{l}\text { Layers of graphite } \\
\text { lattice }\end{array}$ & $\begin{array}{l}\text { Steele's 10-4-3 } \\
\text { Lennard-Jones } \\
+\quad \text { electrostatic } \\
\text { interaction } \\
\text { potential }\end{array}$ & $\begin{array}{l}\text { Partial derivative of system } \\
\text { energy on adsorbed particle } \\
\text { number in Grand canonical } \\
\text { Monte Carlo (GCMC) } \\
\text { simulation plus kT }\end{array}$ \\
\hline $\begin{array}{l}\text { Graphitized } \\
\text { black }+\stackrel{\mathrm{CH}_{3} \mathrm{OH}}{ }, \\
\mathrm{CH}_{3} \mathrm{CH}_{2} \mathrm{OH}[44]\end{array}$ & $\begin{array}{lr}\text { Layers of } & \text { graphite } \\
\text { lattice }+ & \text { parallel } \\
\text { slit-shaped } & \text { pore } \\
\text { model } & \end{array}$ & $\begin{array}{l}\text { Steele's 10-4-3 } \\
\text { Lennard-Jones } \\
+\quad \text { electrostatic } \\
\text { interaction } \\
\text { potential }\end{array}$ & $\begin{array}{l}\text { Partial derivative of system } \\
\text { energy on adsorbed particle } \\
\text { number in Grand canonical } \\
\text { Monte Carlo (GCMC) } \\
\text { simulation plus kT }\end{array}$ \\
\hline
\end{tabular}


In these simulations, simple parallel slit-shaped pore model and Steel's 10-4-3 interaction potential models are commonly used to calculate the adsorbate-adsorbent interaction potential and the external wall potential. The Clausius-Clapeyron equation is then applied to the Henry's region to develop a deterministic relationship between the isosteric heat and the pore width.

For calculating the isosteric heat of adsorption at higher pressures, where adsorbate-adsorbate interactions can no longer be ignored, a GCMC simulation based on probabilistic method is widely applied. Therefore, to construct a more structured model than the simple slit-shaped pore model is necessary.

\subsection{Adsorption Assisted Cooling and Desalination}

Generally, an adsorption assisted cooling and desalination system will consist of evaporators, adsorption and desorption beds, condenser and auxiliary subsystems as shown in Figure 2.1

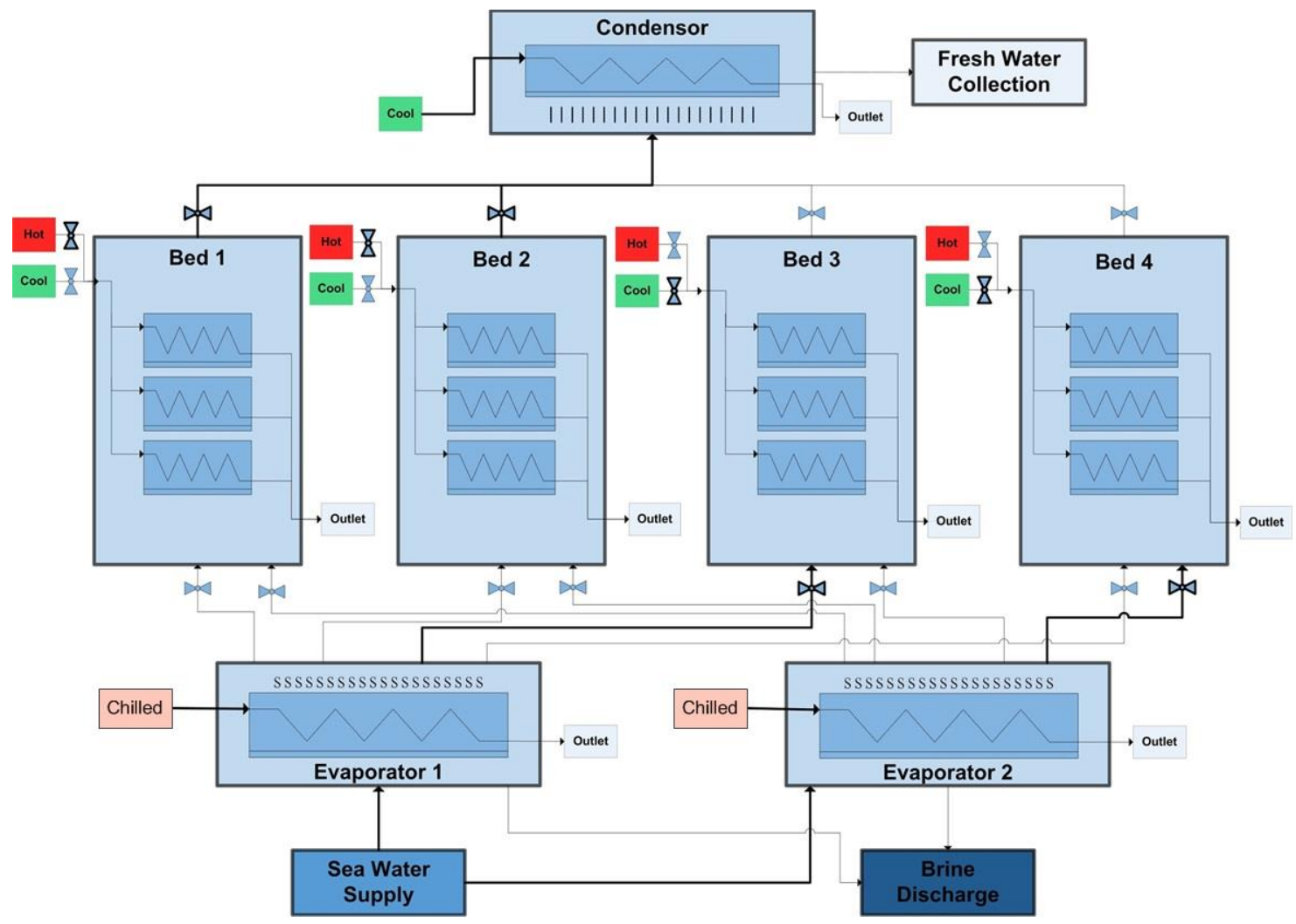

Figure 2.1 Schematic of adsorption cooling and desalination system 
Employing silica gel and water as the working pair, liquid water is firstly vaporised in the evaporation chamber with the heat from a heat exchanger inside it. The generated water vapour is then charged into the adsorption bed during the adsorption process. Water vapour is then adsorbed at the adsorption bed by adsorbent. The adsorption/desorption temperature is maintained by an internal heat exchange subsystem with cold/hot water supply. After a specified half-cycle, the adsorption process is switched off and then the desorption process starts. Similar to adsorption process, adsorbed water in the previous adsorption half-cycle is released from the adsorbent. After another half-cycle, the desorption process is switched off and then the adsorption process starts again. During the switching period, the system will reverse the cooling and hot water supply so as to preheat and precool the beds for the next half-cycle. On the other hand, the released water vapour at desorption process is charged into the condenser with an internal heat exchange subsystem. Water vapour is cooled, condensed to liquid water and finally collected in a water collection tank. These two adsorption and desorption half-cycles together with two switching periods become a whole working cycle for the adsorption assisted cooling and desalination cycle.

The performance parameter of adsorption cooling cycle is defined by the coefficient of performance (COP) i.e. $\left(Q_{\text {evap }} / Q_{\text {des }}\right)[10]$, where $Q_{\text {evap }}$ and $Q_{\text {des }}$ are cooling capacity and the amount of heat added to the sorption bed during desorption stage. The overall performance ratio (OPR) is given by $\left(Q_{\text {evap }}+Q_{\text {cond }}\right) / Q_{\text {des }}[45]$. The specific cooling power (SCP) is the ratio of the cooling power to the total mass of adsorbent (i.e. $Q_{\text {evap }} / M_{S}$ in unit: $\mathrm{W} / \mathrm{kg}, M_{S}$ is the mass of adsorbent) [46]. The performance for adsorption desalination system is defined by specific water production (SWP) and Specific Daily Water Production (SDWP). SWP is calculated as ( $q_{a d s}-$ 
$\left.q_{d e s}\right)$ and $S D W P=\int_{0}^{t_{c y c l e}} \frac{Q_{\text {cond }}}{h_{f g} M_{S}} d t$, where $t_{c y c l e}$ is the cycle time, $Q_{c o n d}$ is condensation heat of the condenser and $h_{f g}$ is the enthalpy of adsorbate[47].

The adsorption cycle has been investigated and applied in cooling systems by many researchers because it is environmentally friendly, free of moving parts, scalability etc. In a conventional adsorption chiller, both the macro and micro-porous silica gels are housed in the copper-fin-tube heat exchanger of adsorption bed to improve the heat and water vapour transfer [48]. Therefore the cooling capacity and COP are increased. The cascade cooling cycle can also be designed by combining two adsorption cooling cycles of zeolite and silica gel as adsorbents respectively [49]. By replacing water with saline in the evaporator, the adsorption cooling cycle can be integrated with desalination and thus creates the Adsorption Desalination (AD) cycle. The advanced AD cycle, an improved adsorption cycle where the heated water in internal heat exchanger of condenser is circulated to that of the evaporator to recover the condensation heat, has been proposed to increase the Specific Daily Water Production (SDWP) of an adsorption desalination system $[3,17,47]$. The mathematical models are proposed based on the isotherm and kinetics of the adsorbent/adsorbate pair to simulate the energy and mass balances for the each component of the cycle [4]. A numerical model on advanced adsorption desalination cycle has been developed by [50] to analyse the system performance.

Adsorption can also be combined with other cooling technologies such as $\mathrm{CO}_{2}$ cooling as gas cooler to recover waste heat. The simulation and experiments of the combined cooling cycles are reported to have a higher COP than only a single cooling cycle [51, 52]. Adsorption is designed to combine with solar energy as a refrigeration cycle at tropical climate [53]. In one study by Habib et al. [54], the adsorption refrigeration comprises two cycles, where the bottoming cycle 
of activated carbon (AC)-R507A is combined with the topping cycle of AC-R134a and the novel refrigeration cycle can provide $-10{ }^{\circ} \mathrm{C}$ cooling temperature at the bottoming cycle.

\subsubsection{Adsorption Cooling}

A review of adsorption cooling systems utilising various adsorbent+ adsorbate pairs, system configuration, adsorption isotherm, adsorption kinetic together with system performances by simulation and experimental investigations are summarised in Table 2.4 The Cooling performances of these systems have been studied with various adsorbent+ adsorbate pairs such as silica gel + water, zeolite + water, zeolite + methanol, $\mathrm{CaCl}_{2} /$ activated carbon + ammonia, activated carbon $+\mathrm{CO}_{2}$, silica gel/LiCl + methanol, and novel composite adsorbent + water.

The theoretical research on adsorption cooling devices currently focuses on mathematical modelling including lumped and distributed modelling that can accurately depict system status at given operational conditions. Optimal operation parameters can be discovered in the optimisation of the cooling performance based on the system modelling. Furthermore, the modelling research can be verified by experimental investigations and also guide the design of adsorption cooling systems [55]. 
Table 2.4 Summary of Adsorption Cooling Systems

\begin{tabular}{|c|c|c|c|}
\hline $\begin{array}{l}\text { Adsorbent+adsorbate } \\
\text { pair }\end{array}$ & Description & Characteristics & Performance \\
\hline $\begin{array}{l}\text { Silica gel( RD type) } \\
\text { +water[56] }\end{array}$ & $\begin{array}{l}\text { Two bed adsorption } \\
\text { chiller simulation } \\
\text { with lumped } \\
\text { modeling }\end{array}$ & $\begin{array}{l}\text { Modified Henry's } \\
\text { isotherm and } \\
\text { LDF kinetic equations }\end{array}$ & $\begin{array}{l}\text { Lumped modelling is } \\
\text { less accurate than the } \\
\text { distributed modelling } \\
\text { and the difference is } \\
\text { less than } 10 \% \text { in } \\
\text { temperature profile }\end{array}$ \\
\hline $\begin{array}{l}\text { Silica gel (RD type and } \\
\text { A type)+water[57] }\end{array}$ & $\begin{array}{l}\text { Two bed adsorption } \\
\text { chiller. } \\
\text { Distributed } \\
\text { modelling is } \\
\text { applied }\end{array}$ & $\begin{array}{l}\text { Tóth isotherm and } \\
\text { near-exponential } \\
\text { kinetics [58] }\end{array}$ & $\begin{array}{l}\text { Smaller grain size and } \\
\text { layer with higher COP }\end{array}$ \\
\hline $\begin{array}{l}\text { Silica gel( } \mathrm{RD} \text { type and } \\
\mathrm{CaCl}_{2} \text { in silica gel ) } \\
\text { +water[59] }\end{array}$ & $\begin{array}{l}\text { Two bed adsorption } \\
\text { chiller with lumped } \\
\text { + distributed } \\
\text { modelling }\end{array}$ & $\begin{array}{l}\text { Modified Freundlich } \\
\text { isotherms for RD type } \\
\text { silica gel plus water. } \\
\text { Tóth isotherm for } \\
\mathrm{CaCl}_{2} \text { in silica gel } \\
\mathrm{LDF} \text { kinetics model }\end{array}$ & $\begin{array}{l}\text { Temporal history of } \\
\text { the AD chiller. } \\
\text { Cooling capacity is } \\
\text { increased by } 6 \%\end{array}$ \\
\hline Zeolite +water $[60,61]$ & $\begin{array}{l}\text { Two bed adsorption } \\
\text { chiller } \\
\text { Lumped modeling } \\
\text { Experimental } \\
\text { investigation }\end{array}$ & $\begin{array}{l}\text { Equilibrium uptake } \\
\text { LDF kinetics model }\end{array}$ & $\begin{array}{l}\text { COP ranges from } 0.1 \\
\text { to } 0.6 \\
\text { Optimal operation } \\
\text { temperature and cycle } \\
\text { time are obtained for } \\
\text { best COP }\end{array}$ \\
\hline Zeolite+ methanol[62] & $\begin{array}{l}\text { Adsorption chiller } \\
\text { analysis with } \\
\text { experimental } \\
\text { investigation }\end{array}$ & $\begin{array}{l}\text { Various operation } \\
\text { conditions to test the } \\
\text { potential application }\end{array}$ & $\begin{array}{l}\text { Temperature profile } \\
\text { and specific cooling } \\
\text { power are obtained } \\
\text { COP ranges from } 0.1 \\
\text { to } 0.12\end{array}$ \\
\hline $\begin{array}{l}\mathrm{LiCl} \text { in silica } \\
\text { gel+methanol[63] }\end{array}$ & $\begin{array}{l}\text { Two bed adsorption } \\
\text { chiller is } \\
\text { experimentally } \\
\text { investigated }\end{array}$ & $\begin{array}{l}\text { Employing both heat } \\
\text { and mass recoveries }\end{array}$ & $\begin{array}{l}\text { The SCP and the } \\
\text { COP are improved by } \\
16.3 \% \text { and } 24.2 \% \text { as } \\
\text { compared with silica } \\
\text { gel+water assisted } \\
\text { chiller }\end{array}$ \\
\hline $\begin{array}{l}\text { Activated carbon } \\
\text { fiber+ethanol[64] }\end{array}$ & $\begin{array}{l}\text { Two bed adsorption } \\
\text { chiller } \\
\text { Lumped modeling }\end{array}$ & $\begin{array}{l}\text { Dubinin- } \\
\text { Radushkevich (D-R) } \\
\text { isotherm and } \\
\text { LDF kinetics model }\end{array}$ & $\begin{array}{l}\text { Temperature profile } \\
\text { and Dühring diagram } \\
\text { are depicted }\end{array}$ \\
\hline $\begin{array}{l}\text { Activated carbon }+\mathrm{CO}_{2} \\
\text { [65] }\end{array}$ & $\begin{array}{l}\text { Four bed adsorption } \\
\text { cooling } \\
\text { Lumped modeling }\end{array}$ & $\begin{array}{l}\text { Tóth isotherm and } \\
\text { experimental data } \\
\text { LDF kinetics models }\end{array}$ & $\begin{array}{l}\text { Temperature profiles } \\
\text { with P-T-X and } \mathrm{P}-h \\
\text { diagram COP against } \\
\text { cycle time }\end{array}$ \\
\hline
\end{tabular}




\begin{tabular}{|c|c|c|c|}
\hline $\begin{array}{l}\mathrm{CaCl}_{2} \text { /activated carbon } \\
+ \text { ammonia } \\
\text { Silica } \\
\text { gel/LiCl+methanol [55] }\end{array}$ & $\begin{array}{l}\text { Two bed } \\
\text { refrigerator } \\
\text { Two bed adsorption } \\
\text { chiller and } \\
\text { experimental } \\
\text { investigation }\end{array}$ & $\begin{array}{l}\text { Constant evaporating } \\
\text { temperature } \\
\text { Both heat and mass } \\
\text { recoveries methods }\end{array}$ & $\begin{array}{l}\text { Clausius Clapeyron } \\
\text { diagram of } \\
\mathrm{CaCl} 2 / \text { activated- } \\
\text { ammonia for } \\
\text { adsorption } \\
\text { refrigeration. } \\
\text { P-T-X diagram of } \\
\text { silica gel/LiCl- } \\
\text { methanol adsorption } \\
\text { chiller }\end{array}$ \\
\hline $\begin{array}{l}\text { Novel composite } \\
\text { adsorbent (activated } \\
\text { carbon soaking in } \\
\mathrm{Na}_{2}\left(\mathrm{SiO}_{2}\right)_{\mathrm{n}} \mathrm{O} \text { and } \mathrm{CaCl}_{2} \\
\text { solution) + water[66] }\end{array}$ & $\begin{array}{l}\text { Descriptions of two } \\
\text { bed adsorption } \\
\text { chiller with } \\
\text { experimental } \\
\text { investigation }\end{array}$ & $\begin{array}{l}\text { Isotherm from } \\
\text { experimental } \\
\text { result[67] } \\
\text { LDF kinetics model is } \\
\text { employed }\end{array}$ & $\begin{array}{l}\text { Composite adsorbents } \\
\text { are used for the } \\
\text { average SCP of } 378 \\
\text { W/kg and the COP of } \\
0.7\end{array}$ \\
\hline
\end{tabular}

\subsubsection{Adsorption Desalination}

Adsorption desalination results from the unique union of evaporation and condensation through adsorption and desorption. A review of adsorption desalination systems with various adsorbent + adsorbate pairs, system configurations, and dynamic performances is summarised in Table 2.5 The performances of various adsorption desalination systems are specified by the performance ratio (PR) and specific daily water production (SDWP).

Table 2.5 Summary of Adsorption Desalination Systems

\begin{tabular}{|l|l|l|l|}
\hline $\begin{array}{l}\text { Adsorbent +adsorbate } \\
\text { pairs }\end{array}$ & Descriptions & Characteristics & Performances \\
\hline $\begin{array}{l}\text { Silica gel (RD type) } \\
\text { +water [68] }\end{array}$ & $\begin{array}{l}\text { Two bed } \\
\text { adsorption chiller } \\
\text { Desalination } \\
\text { employing } \\
\text { distributed } \\
\text { modelling and } \\
\text { experimental } \\
\text { investigations }\end{array}$ & $\begin{array}{l}\text { Linear } \\
\text { adsorption } \\
\text { isotherm[69] } \\
\text { LDF kinetics }\end{array}$ & $\begin{array}{l}\text { Modelling is validated with } \\
\text { experimental data for errors } \\
\text { less than 5\% } \\
\text { Optimal cycle time is identified } \\
\text { for best specific water } \\
\text { production }\end{array}$ \\
\hline $\begin{array}{l}\text { Silica gel (RD type) } \\
\text { +water [70] }\end{array}$ & $\begin{array}{l}\text { Four bed } \\
\text { adsorption chiller } \\
\text { Two stage } \\
\text { Lumped } \\
\text { modelling }\end{array}$ & $\begin{array}{l}\text { Tóth isotherm } \\
\text { LDF kinetics }\end{array}$ & $\begin{array}{l}\text { Novelty of inter-stage pressure } \\
\text { as optimisation parameter }\end{array}$ \\
\hline
\end{tabular}




\begin{tabular}{|c|c|c|c|}
\hline $\begin{array}{l}\text { Silica gel }\left(\mathrm{A}^{++} \text {type }\right)+ \\
\text { water }[4,17,71]\end{array}$ & $\begin{array}{l}\text { Four bed } \\
\text { advanced AD } \\
\text { cycle with heat } \\
\text { recovery* } \\
\text { Lumped } \\
\text { modelling }\end{array}$ & $\begin{array}{l}\text { Dubinin- } \\
\text { Astakhov (D-A) } \\
\text { adsorption } \\
\text { isotherm } \\
\text { modelling }\end{array}$ & $\begin{array}{l}\text { SDWP to hot water/cooling } \\
\text { water inlet temperature } \\
\text { SDWP varies from } 8.1 \text { to } 26 \\
\mathrm{~m}^{3} \text { per tonne of silica gel per } \\
\text { day }\end{array}$ \\
\hline Silica gel + water [50] & $\begin{array}{l}\text { Two bed AD } \\
\text { cycle integrated } \\
\text { with evaporator } \\
\text { and condenser } \\
\text { Lumped } \\
\text { modeling }\end{array}$ & $\begin{array}{l}\text { Dubinin- } \\
\text { Astakhov (D-A) } \\
\text { LDF kinetics } \\
\text { equation }\end{array}$ & $\begin{array}{l}\text { Temperature, pressure profile } \\
\mathrm{P}-\mathrm{T}-\mathrm{C} \text { diagram of AD cycle } \\
\text { The SDWP against cycle time, } \\
\text { varies from } 8.1 \mathrm{~m}^{3} \text { to } 26 \mathrm{~m}^{3}\end{array}$ \\
\hline Silica gel +water [72] & $\begin{array}{l}\text { Two bed and four } \\
\text { bed mode } \\
\text { Experimental } \\
\text { investigation }\end{array}$ & $\begin{array}{l}\text { Tóth isotherm } \\
\text { and LDF } \\
\text { kinetics model }\end{array}$ & $\begin{array}{l}\text { Optimal performance } \\
\text { parameters such as SDWP and } \\
\text { PR are simulated and } \\
\text { compared with experimental } \\
\text { data }\end{array}$ \\
\hline $\begin{array}{l}\text { Silica gel + water } \\
\text { MED [73-75] }\end{array}$ & $\begin{array}{l}\text { Two bed AD } \\
\text { cycle hybrid with } \\
\text { Multi Effect } \\
\text { Distillation } \\
\text { (MED) } \\
\text { Lumped } \\
\text { modelling }\end{array}$ & $\begin{array}{l}\text { Tóth isotherms } \\
\text { and LDF } \\
\text { kinetics models } \\
\text { for simplicity }\end{array}$ & $\begin{array}{l}\text { MED + AD increases water } \\
\text { production more than } 3 \text { folds } \\
\text { as compared to traditional } \\
\text { MED plants. }\end{array}$ \\
\hline
\end{tabular}

*In an advanced adsorption desorption (AD) desalination cycle, the internal latent heat between

the evaporator and the condenser units is recovered and also is equivalent to that with a compressor to pressurise the adsorbent during adsorption, and thus making the adsorbent with a higher water vapour uptake [47].

It has been pointed out that the conventional desalination devices such as Multi-Stage Flash (MSF), Multi-Effect Desalination (MED), solar distillation, reverse osmosis (RO) and the electro-dialysis suffer from desalination shortcomings with the difficulties of maintenance, relatively high energy consumption and also high cost [26]. The water quality of adsorption desalination process is independent of high feed salinity in the evaporator and the chemical quality of desalinated water is comparable to deionized water quality [76]. Adsorption desalination can meet the need for desalination systems with low energy and running cost. 


\subsection{Summary}

In this chapter, the literatures related to the proposed research objectives and scope are reviewed. The adsorption phenomena together with adsorption isotherms and kinetics models are introduced to offer a clear theoretical and empirical development for the quantitative prediction of adsorption isotherms and kinetics, which will later be applied in the modelling and simulation of isosteric heat as well as adsorption chiller. The widely applied pore models, interaction potentials models for various adsorbent-adsorbate pairs in the simulation of isosteric heat are listed. Several novel cooling and desalination technologies are introduced to highlight the advantages of adsorption process regarding the environmental and economic concerns about cooling and desalination systems. Finally, the descriptions and performances of some adsorption cooling and desalination systems are presented to prove the potential applications of adsorption. The next chapter begins with a theoretical insight of isosteric heat at zero coverage. 


\section{Chapter 3 Modelling and Simulation of Isosteric Heat of Adsorption at Zero Coverage}

\subsection{Introduction}

Generally, the modelling of the adsorbate and adsorbent structures are needed in the first place in order to calculate the interaction potential and isosteric heat of adsorption at zero coverage $\left(q_{s t}^{o}\right)$. These parameters can also be expressed as a function of pore width. All these information are needed to design adsorption beds for adsorption cooling, desalination or gas storage [13, 77-82]. In this chapter, the adsorbents of lattice, amorphous and framework structures are described to understand the nature of adsorption interactions with various adsorbate molecules.

Graphitised carbon has a honeycomb lattice with a simple pore structure. It is well known that the potential energy and $q_{s t}^{o}$ provide important information about the adsorption mechanism ranging from Henry's region to the saturated pressure. Unlike covalent-network solids such as graphite, silica has an amorphous structure without a periodic, crystal-like surface such as silica gel. Silica gel is a form of highly porous amorphous silica $\left(\mathrm{SiO}_{2}\right)$ and is made from anhydride of silicic acid. Amorphous silica $\left(\mathrm{a}-\mathrm{SiO}_{2}\right)$ is formed with $\mathrm{Si}\left(\mathrm{O}_{1 / 2}\right)_{4}$ tetrahedrons which are connected by oxygen atoms [83]. Unlike the quartz crystal with a repeated unit cell in the molecular structure, the number of $\mathrm{Si}$ atoms in the ring of $\mathrm{Si}\left(\mathrm{O}_{1 / 2}\right)_{4}$ tetrahedrons may differ from 2 to 8 due to the amorphicity of silica [84-86]. The length of the Si-O covalent bond is $1.61 \AA$ [87] and its dipole moment is $3.098 \mathrm{D}$ [88]. The difference between $\mathrm{Si}$ and $\mathrm{O}$ atoms is less than 1.7 in the Pauling electronegativity scale. The structure also possesses $50 \%$ of ionic character and $50 \%$ covenant character of $\mathrm{Si}-\mathrm{O}$ bond. It should be noted here that framework structures such as zeolites [89, 90] are three-dimensional, highly porous, crystalline inorganic materials. The 
structural instability of alumino-phosphate zeolite formed by $\mathrm{TO}_{4}$ tetrahedral $(\mathrm{T}=\mathrm{Al}, \mathrm{P}$ in an alternative manner) has limited its applications [91]. Therefore, other elements such as $\mathrm{Si}$ and $\mathrm{Fe}$ are incorporated into alumino-phosphate framework to generate more stable silico-aluminophosphate, ferroaluminophosphate, etc. [92]

In this chapter, a thermodynamic framework is presented to calculate the binding energies of adsorbate molecules on various adsorbent structures such as graphite basal plane, amorphous silica gel and CHA and AFI type zeolite framework structures. The proposed modelling employs the static molecular model, where the Lennard-Jones (LJ), electrostatic and induction potentials are considered. The potential energy surfaces are presented with respect to various orientations of polar adsorbate molecules. Employing the derived potential model, the isosteric heat of adsorption is calculated as a function of pore width $H$.

\subsection{Previous Studies on Adsorbent Structures and Modellings}

Adsorption of gases and liquids on graphite surfaces was extensively studied [13, 80-82] as the system comprising graphite and polar or non-polar adsorbate provides a bench mark to evaluate molecular models such as Grand Canonical Monte Carlo (GCMC) and molecular dynamics (MD). However these methods are complex and require very high computational speed to calculate the amount of adsorbate uptake, kinetics and isosteric heat of adsorption. Therefore, for simplicity, a static molecular simulation technique will be applied to calculate the interaction potential and the isosteric heat of adsorption at zero coverage $\left(q_{s t}^{o}\right)$.

The interactions of adsorbate molecules on a graphite surface are analysed by intermolecular potential models to determine the binding energies [80]. Among the basic intermolecular potential models, the Lennard-Jones (LJ) potential model of molecules with weak long range attraction and strong short range repulsion [93] has been applied to predict the maximum binding 
energies. The pair-wise additive L-J potential model between oxygen and carbon has been widely employed to the simulation of molecular dynamics of water and graphite systems [80]. Besides the interactive force between adsorbate and adsorbent molecules, the lateral attraction force between adsorbed molecules is reported to be positively strong on most homogenous, graphitised carbon surfaces whether the adsorbate molecule is polar or non-polar [94].

The Continuous Random Network (CRN) model was firstly proposed to describe the structure of amorphous semiconductors [95]. Based on the CRN model, the construction of amorphous silica structure was made from the diamond structure with periodic boundary conditions by Wooten, Winer and Weaire $[83,96,97]$, which is known as WWW method. By randomly and repeatedly transposing the $\mathrm{Si}-\mathrm{Si}$ bond in amorphous $\mathrm{Si}$, a continuous random network can be generated while the tetrahedronal coordination of each atom is preserved. The crystalline state is partially preserved after random operations due to the memory of initial configuration according to WWW model [98]. Therefore, a structural modelling technique namely Reverse Monte Carlo (RMC) method based on experimental data is developed to describe the structure of noncrystalline materials [99]. The RMC method is applied to the model of the molecular structures of amorphous silica [100] and silicon [101], which are consistent with available experimental data.

The adsorption of water onto silica gel $\left(\mathrm{SiO}_{2} \cdot \mathrm{nH}_{2} \mathrm{O}\right)$ is considered as the interaction of $\mathrm{H}_{2} \mathrm{O}$ molecule with the surface of amorphous silica structure for simplicity. For non-bonded interaction, the van der Waals and the electrostatic interactions are the main effects to determine the interaction potential. The interaction of $\mathrm{H}_{4} \mathrm{SiO}_{4}-\mathrm{H}_{2} \mathrm{O}$ pair is studied and applied to the molecular dynamics simulation to understand the adsorption of water on silica surface [82]. The residual hydroxyl group on the surface of silica is also considered in these simulation studies [96, 
102]. The Born-Mayer-Huggins pair-wise potential model is applied in the simulation of adsorption of water on silica and silicate glass fracture surfaces [103]. It should be noted here that the calculation of $q_{s t}^{o}$ for the adsorption of water on CHA and AFI type zeolites such as AQSOA-Z01, AQSOA-Z02 and AQSOA-Z05 are not available in the literature. In the next section, the modelling of $q_{s t}^{o}$ based on molecular dynamics is presented in detail.

\subsection{Molecular Models}

The adsorption potential model integrates LJ, electrostatic and induction potentials, which takes into account dipole-dipole, dipole-quadrupole and dipole-induced dipole interactions. Therefore, the predicted interaction potential is more accurate than those that consider only LJ and chargecharge interactions according to the energy expansion equation. Previously, the multilayer LJ potential equation was applied as the interaction potential equation to predict the $q_{s t}^{o}$ of adsorption for non-polar molecules in the parallel slit pore model [13, 104]. Instead of the widely adopted approximation of multilayer LJ potential [105], the present work applies the integrated and deterministic interaction potential. For simplicity, the parallel slit model is used for predicting $q_{s t}^{o}$ as a function of pore width for some specific orientations. The interaction between adsorbate molecules is as important as that between adsorbate and adsorbent molecules to calculate the isosteric heats. However, this research work focuses more on $q_{s t}^{o}$ at zero coverage surfaces. Therefore, the Henry's law constant is employed in the pressure-temperature-uptake co-ordinate system (Clausius-Clapeyron equation) for the calculation of $q_{s t}^{o}$. At zero surface coverage, the density of the adsorbed adsorbate molecules in the simulation box is relatively low, which allows the negligible adsorbate-adsorbate interactions. 


\subsubsection{Molecular Model of Lattice Surface}

In physisorption, the interactions between adsorbed species and the adsorbent surface occurrs due to weak van der Waals and electrostatic-multipole forces. The total interaction potential is fairly low $(\leq 0.5 \mathrm{eV})$. A single component adsorbate molecule physisorbed on a graphite plane is considered for simplicity. Figure 3.1 shows the geometrical characteristics of a $\mathrm{H}_{2} \mathrm{O}$ molecule adsorbed on the hexagonal graphite surface, which contains three particular orientation sites namely the hexagonal centre, C-C bond and carbon atoms. The selected simulation box comprises 10 layers of carbon atoms and each layer includes $41 \times 41$ carbon atoms. The sampling adsorbate molecule can move freely. For a given set of carbon atoms $r_{j}$, the LennardJones, electrostatic and induction potentials are only functions of adsorbent molecule position vector $\mathbf{r}$ or $(X, Y, Z)$ in graphite co-ordinate system. The carbon atom layers are in $X-Y$ plane with

fixed $Z$, the direction of adsorbate molecule to the honey-comb lattice of graphite. The total interaction potential $(U)$ depends on the relative position of adsorbate molecule to the hexagonal unit cell of graphite. With fixed $X$ and $Y$, the total interaction potential depends on the $Z$ direction. 


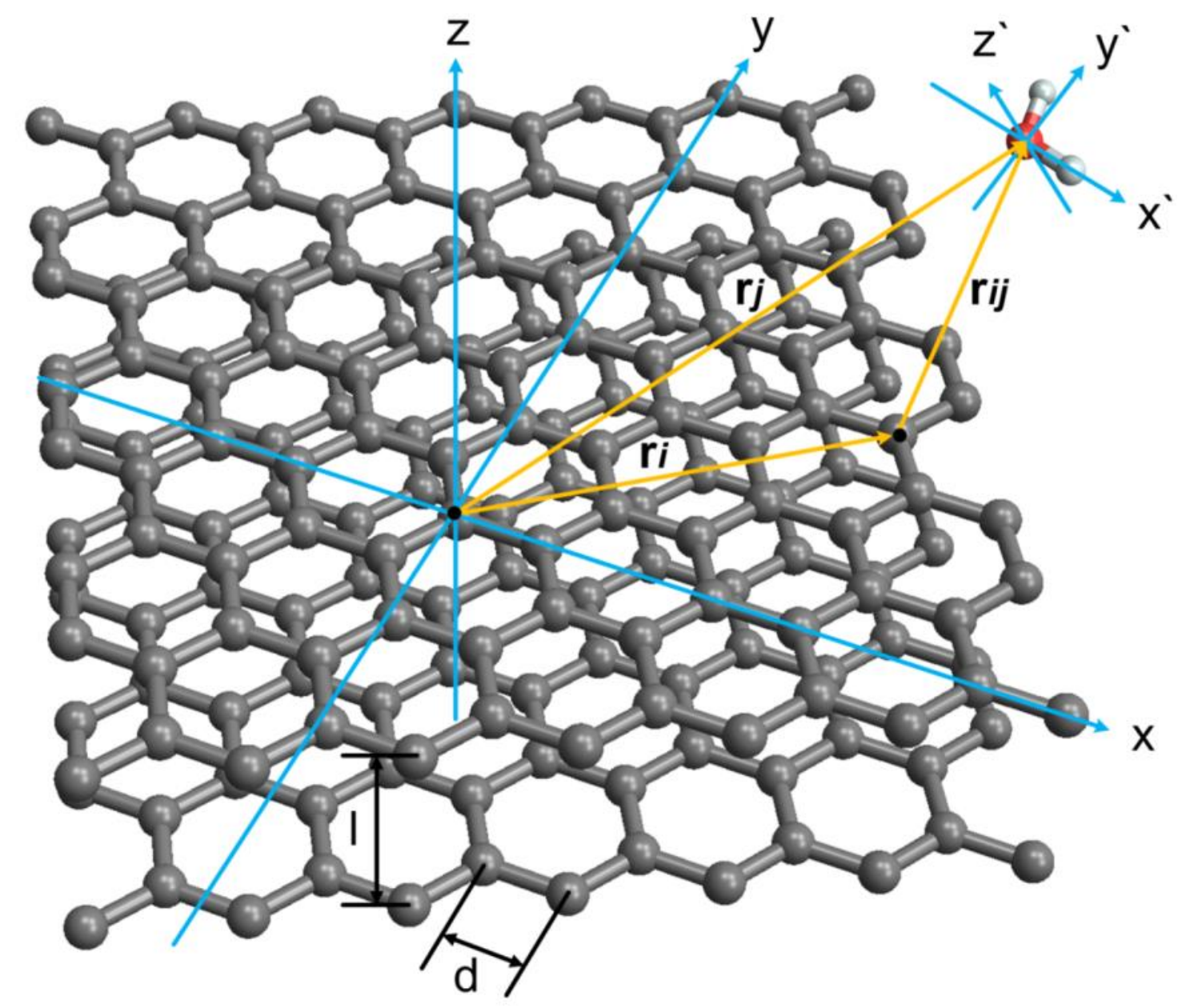

Figure 3.1 The simulation model of graphite and water molecule (here $\mathrm{H}_{2} \mathrm{O}$ is considered as an example of polar molecule). The edge length of the hexagon of carbon atoms is $d=1.421 \AA$ and the distance of two layers is $\mathrm{I}=3.354 \AA$. The position vector points from the $\mathrm{i}^{\text {th }}$ carbon to the $\mathrm{j}^{\text {th }}$ atom of the adsorbate molecule. Position vector in adsorbate molecule coordinate system $X^{\prime} Y^{\prime} Z^{\prime}$ can be converted to graphite coordinate system $X Y Z$ with a specific rotational matrix and translational vector.

\section{Interaction Potential of Lattice Surface and Adsorbate Molecules}

Combining the L-J, electrostatic and induction potentials, the total interaction potential $\left(U_{m M}\right)$ term is used to obtain a more complete description of interaction potential for polar molecules, i.e., $U_{m M}=U_{L J}+U_{E}+U_{I}$. The modified L-J potential model is given by [80] 
$\mathrm{U}_{\mathrm{LJ}}\left(\boldsymbol{r}_{\boldsymbol{i}}-\boldsymbol{r}_{\boldsymbol{j}}\right)=4 \varepsilon_{i j}\left\{\left(\frac{\sigma_{i j}}{\left|\boldsymbol{r}_{\boldsymbol{i}}-\boldsymbol{r}_{\boldsymbol{j}}\right|}\right)^{12}\left[1+\gamma_{R}\left(1-\frac{6}{5} \cos ^{2} \theta\right)\right]-\left(\frac{\sigma_{i j}}{\left|\boldsymbol{r}_{\boldsymbol{i}}-\boldsymbol{r}_{\boldsymbol{j}}\right|}\right)^{6}\left[1+\gamma_{A}\left(1-\frac{3}{2} \cos ^{2} \theta\right)\right]\right\}$,

where $\boldsymbol{r}_{\boldsymbol{i}}$ indicates the position vector of $i$ th adsorbate molecule atom, $\boldsymbol{r}_{\boldsymbol{j}}$ is the position vector of the $j$ th carbon atom, $\varepsilon_{i j}$ and $\sigma_{i j}$ are pair well depth potential and collision diameter, $\gamma_{R}$ and $\gamma_{A}$ are coefficients for repulsion and dispersion component of L-J potential, $\theta$ is the angle between position vector $\left(\boldsymbol{r}_{\boldsymbol{i}}-\boldsymbol{r}_{\boldsymbol{j}}\right)$ and outer normal vector of carbon atom plane. According to the Lorentz-Berthelot combining rules, $\varepsilon_{i j}=\sqrt{\varepsilon_{i} \varepsilon_{j}}$ and $\sigma_{i j}=\frac{\sigma_{i}+\sigma_{j}}{2}$. Equation (3.1) also captures the anisotropic behaviours of graphite. The carbon atom possesses quadrupole polarisability [106] while the dipole moment effects cannot be neglected for polar molecules such as water. The interaction potential of polar molecules with graphite adsorbent consists of charge-charge as well as high-order interactions according to the energy expansion equation [107]. Therefore, the necessity for high-order effects such as dipole-dipole, dipole-quadrupole and induction in polar molecule adsorption is palpable. In the two-body interaction, the polarisation arises from the electric field formed by the opposite source. The strength of the polarisation is characterized by the dipole and quadrupole moment polarisability tensor. Similarly, the transient induced dipole moment is excited by the opposing electric field to a permanent dipole moment. The electrostatic potential between water molecule and carbon atom as shown in Figure 3.2 is derived from the interaction between polar molecule, e.g., water dipolar moment $\boldsymbol{\mu}$ and quadrupole moment $\boldsymbol{\Theta}$ in the electric field $\mathbf{E}\left(\mathbf{r}-\mathbf{r}_{\mathbf{j}}\right)$ exerted by the carbon atom.

$$
\mathrm{U}_{\mathrm{E}}\left(\mathbf{r}-\mathbf{r}_{\mathbf{j}}\right)=\boldsymbol{\mu} \cdot \mathbf{E}\left(\mathbf{r}-\mathbf{r}_{\mathbf{j}}\right)+\frac{1}{3} \boldsymbol{\Theta}: \nabla \mathbf{E}\left(\mathbf{r}-\boldsymbol{r}_{\mathbf{j}}\right)
$$

where $\mathbf{r}$ is the position vector for the mass centre of water molecule, $\mathbf{r}_{\mathbf{j}}$ is the position vector of jth carbon atom. The first term of the right hand side indicates the dipolar component of electrostatic potential, which is the inner product of dipolar moment vector $\boldsymbol{\mu}$ and the exerted 
electric field $\mathbf{E}\left(\mathbf{r}-\mathbf{r}_{\mathbf{j}}\right)$. The second term of the right hand side is quadrupolar, and this is the double-dot product of quadrupolar moment tensor $\boldsymbol{\Theta}$ and the electric field gradient (EFG) tensor $\boldsymbol{\nabla E}\left(\mathbf{r}-\mathbf{r}_{\mathbf{j}}\right)$. A screening factor, which is 1 for first layer of carbon atoms and $2 /(2.8+1)$ for the rest layers, is applied to the electrical potential of the dipole and quadrupole moment [42].

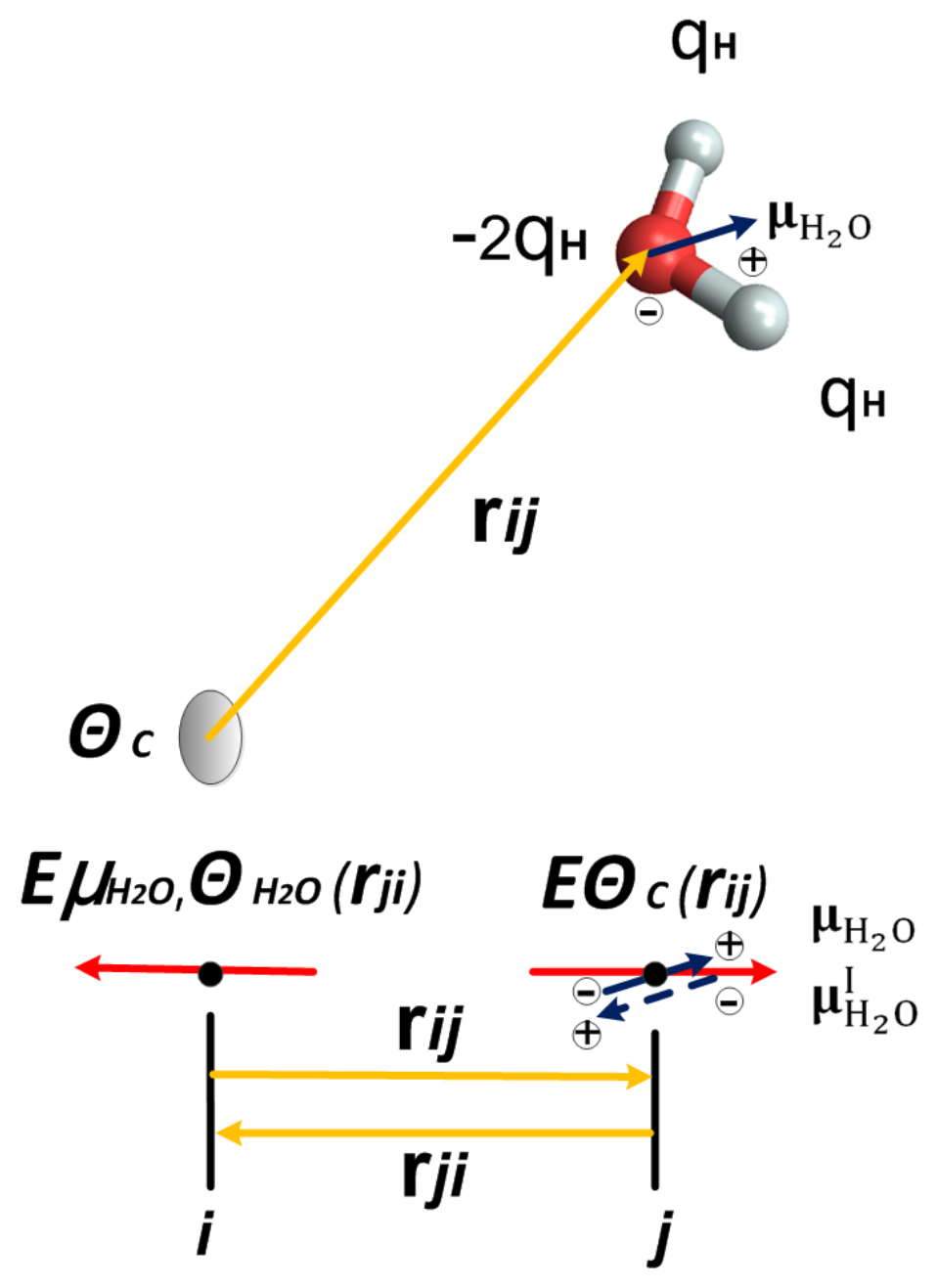

Figure 3.2 Electrostatic model of water and graphite. Here the dipole moment vector $(\mu)$ for water and quadrupole moment matrix $(\Theta)$ for water and carbon are shown. $\boldsymbol{r}_{i j}$ is the position vector from the ith carbon atom to the jth atom of water molecule and $\boldsymbol{r}_{i j}=-\boldsymbol{r}_{j i}$. The positive charge for hydrogen atom is $\mathrm{q}_{\mathrm{H}}$ and the negative charge of oxygen atom is $-2 \mathrm{q}_{\mathrm{H}}$. The electric 
field generated by the quadrupole moment of carbon atom at position $\boldsymbol{r}_{i j}$ is $\operatorname{E} \theta_{\mathrm{C}}\left(\boldsymbol{r}_{i j}\right)$ and the electric filed generated by the dipole and quadrupole moment of water is $\mathrm{E} \mu_{\mathrm{H}^{2} \mathrm{O}} \theta_{\mathrm{H}^{2} \mathrm{O}}\left(\boldsymbol{r}_{i j}\right)$ and $\theta_{\mathrm{H}^{2} \mathrm{O}}$ are the quadrupole moment of carbon and water. $\mu_{\mathrm{H}_{2} \mathrm{O}}$ and $\mu_{\mathrm{H} 2 \mathrm{O}}^{I}$ are the permanent and transient induced dipole moment of water.

The interaction between transient dipole and permanent charge contributes to the interaction potential, and is represented by

$$
\mathrm{U}_{\mathrm{I}}\left(\mathbf{r}-\mathbf{r}_{\mathbf{j}}\right)=-\frac{1}{2} \boldsymbol{\mu}_{\mathbf{p}}^{\mathrm{I}} \cdot \mathbf{E}_{\mathrm{p}}\left(\mathbf{r}-\mathbf{r}_{\mathbf{j}}\right)-\frac{1}{2} \boldsymbol{\mu}_{\mathrm{C}}^{\mathrm{I}} \cdot \mathbf{E}_{\mathbf{C}}\left(\mathbf{r}-\mathbf{r}_{\mathbf{j}}\right)
$$

where $\mathbf{r}$ is the position vector for the mass centre of water molecule, $\mathbf{r}_{\mathbf{j}}$ is the position vector of the $j$ th carbon atom, $\boldsymbol{\mu}_{\mathrm{p}}^{\mathrm{I}}$ and $\boldsymbol{\mu}_{\mathrm{C}}^{\mathrm{I}}$ are the induced dipole moment of polar molecule and carbon that can be calculated according to their polarizability, $\mathbf{E}_{\mathbf{p}}\left(\mathbf{r}-\mathbf{r}_{\mathbf{j}}\right)$ and $\mathbf{E}_{\mathbf{C}}\left(\mathbf{r}-\mathbf{r}_{\mathbf{j}}\right)$ are the total electric fields of polar molecule and carbon atom due to both permanent and transient induced dipole.

From the geometrical and potential model, the interaction potential $\mathrm{U}(\mathrm{z})$ of one adsorbate molecule with the distance $\mathrm{z}$ above the surface of adsorbent can be determined. The parallel slit model is shown in Figure 3.3. By definition, the external well potential as a function of $z$ can be expressed with respect to the total interaction potential, which is given by

$$
\mathrm{V}_{\mathrm{ext}}(\mathrm{z})=\mathrm{U}_{\mathrm{mM}}(\mathrm{z})+\mathrm{U}_{\mathrm{mM}}(\mathrm{H}-\mathrm{z})
$$

where $H-z$ is the distance of adsorbate molecule to the surface of opposite wall, $H=\left(H_{c}-\sigma_{\text {ss }}\right)$ is the required distance for adsorbent-adsorbate interactions along $z$ direction, $\mathrm{H}_{\mathrm{c}}$ is the maximum possible distance between centres of graphite on opposing wall and $\sigma_{\mathrm{ss}}$ is the size of carbon atom. 


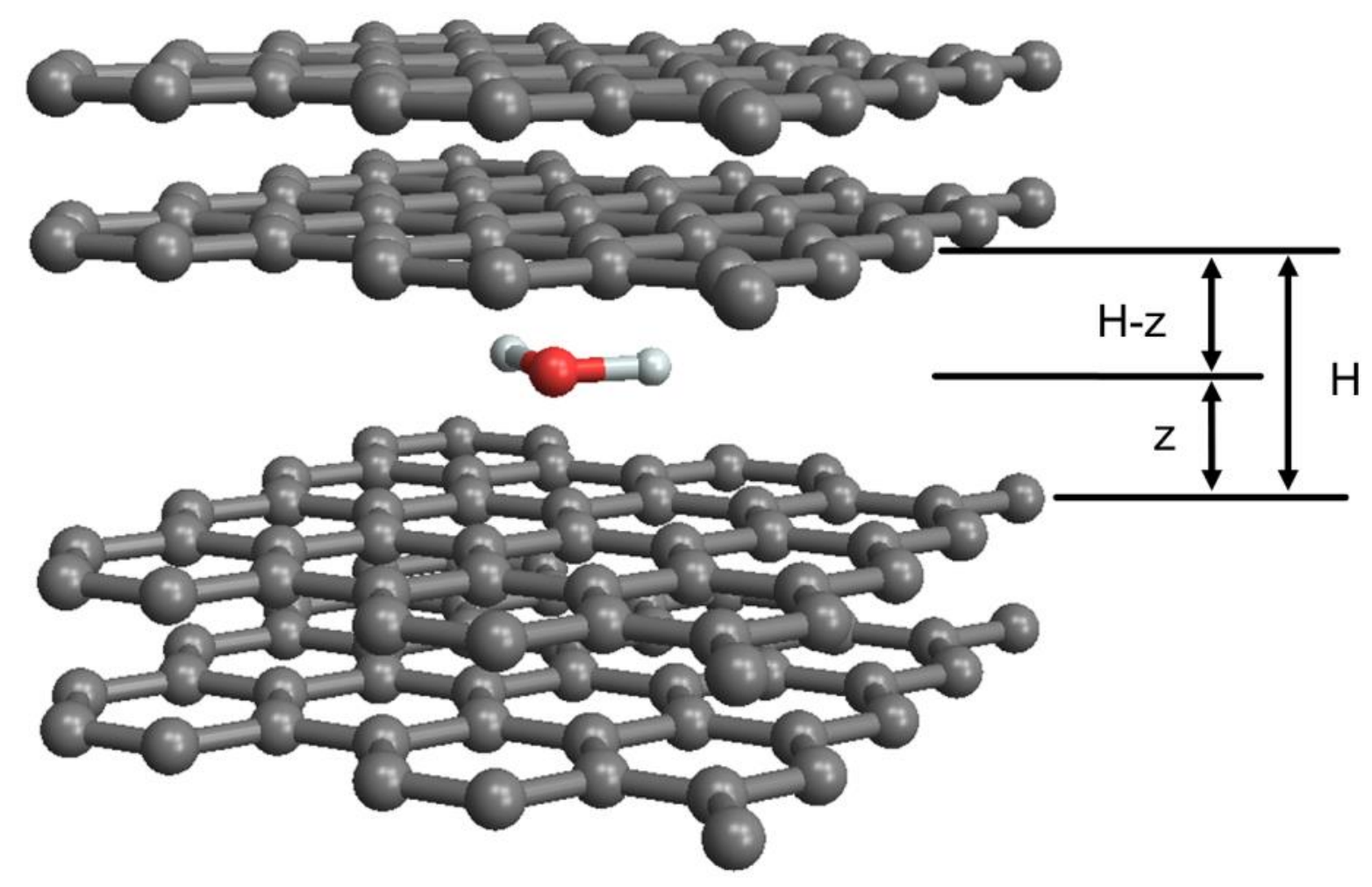

Figure 3.3 The parallel slit shape pore model for graphite. The distance of the centre of adsorbate molecule to the surface of multi-layer graphite atoms and the opposing wall are $z$ and $H-z$ respectively for the pore width of $H$. The external wall potential of the adsorbate molecule in the pore is the sum of the interaction potentials with both walls.

\subsubsection{Molecular Model of Amorphous $\mathrm{SiO}_{2} \mathrm{Surface}$ and Water Molecules}

The model of amorphous silica is the fundamental problem of this research work. Meanwhile, the adsorbent-adsorbate pair interaction potential is required for the computation of isosteric heat of adsorption. Based on the model of silica gel molecular structure and interaction potential, the isosteric heat of adsorption for silica gel and water is determined by applying the Steele's model [105]. For water molecule in TIP3P water model [108], the hydrogen-oxygen-hydrogen angle is $104.5^{\circ}$ and the hydrogen-oxygen bond length is $0.95728 \AA$ with partial charge of $+0.4238 \mathrm{e}$ and 0.8476 e for hydrogen and oxygen atoms, respectively. 
The structure of amorphous silica is considered in this study for simulating and understanding its realistic geometry. Generally, the $\mathrm{Si}\left(\mathrm{O}_{1 / 2}\right)_{4}$ unit is modelled as a tetrahedron with oxygen atoms at the edge and one silicon atom at the centre. At the connection point of the two tetrahedrons, each oxygen atom is shared by two silicon atoms. The amorphicity of the silica structure refers to the uncertainty of the number of silicon atoms in the ring of connected tetrahedrons because the ring can be consisted of 3 to 8 silicon atoms [84].

To generate an amorphous silica network for simulation purposes, the Reverse Monte Carlo (RMC) method is chosen. Hence, we start our analysis with partial radial distribution function as given by [100].

$$
\mathrm{g}_{u v}(r)=\frac{n_{u v}(r)}{4 \rho_{v} \pi r^{2} \mathrm{~d} r}
$$

where $n_{u v}(r)$ is the average number of $v$ atom from $u$ atom between distances of $\mathrm{r}-\mathrm{d} r / 2$ and $\mathrm{r}+\mathrm{d} r / 2, \rho_{v}$ is the mean number density of $v$ atom, $r$ is the distance between atoms, $\mathrm{d} r$ is a small distance as compared with $r$.

The Total Radial Distribution is given by,

$$
\mathrm{G}(r)=4 \pi r \rho_{0}\left[\sum_{u=1, v=u}^{m}\left[w_{u v} g_{u v}(r)\right]-1\right]
$$

where $\rho_{0}$ is total number density of all atoms, and $m$ is the type of all atoms. Take silica for example, $m=2$ for silica as there are two types of atom in silica structure, i.e., $\mathrm{Si}$ and $\mathrm{O}$ atoms. The weighting factor $w_{u v}$ is defined as,

$$
w_{u v}=\left(\sum_{k=1}^{m} c_{k} \bar{b}_{k}\right)^{-2} c_{u} c_{v} \bar{b}_{u} \bar{b}_{v}
$$


where $c_{u}=\rho_{u} / \rho_{0}$ is the molar fractions of atom $\boldsymbol{u}$, and $\bar{b}_{u}$ is the average bound coherent scattering length. Employing experimentally measured X-ray diffraction data in RMC simulation, $\bar{b}_{u}$ is replaced by the atomic form factor $f_{u}$ as shown in Table 3.1

Table 3.1 Average bound coherent scattering lengths and atomic form factor for $\mathrm{Si}$, and $\mathrm{O}$

\begin{tabular}{ccc}
\hline Atom & Si & 0 \\
\hline $\bar{b}$ & 4.1491 & 5.803 \\
$f$ & 14.41 & 8.144 \\
\hline
\end{tabular}

The Structure Factor $S(Q)$ is given by,

$$
\mathrm{S}(\mathrm{Q})=1+\frac{1}{Q} \int_{0}^{\infty} \mathrm{G}(r) \sin (Q r) \mathrm{d} r
$$

The momentum transfer $Q$ is given by,

$$
Q=\frac{4 \pi}{\lambda} \sin \left(\frac{\theta}{2}\right),
$$

where $\lambda$ is the wavelength and $2 \theta$ is the diffraction angle.

The structure factor is then used in the chi-square test to determine the acceptance or rejection of one move in RMC simulation and the chi-square test formulation is given by,

$$
\chi_{S}^{2}=\sum_{i=1}^{N} \frac{\left[\mathrm{S}^{c}\left(\mathrm{Q}_{i}\right)-\mathrm{S}^{e}\left(\mathrm{Q}_{i}\right)\right]^{2}}{\sigma_{S}^{2}},
$$

where $\sigma_{s}$ is the standard error of experimental data, $S^{e}\left(Q_{i}\right)$ is the structure factor for experimental data and $S^{c}\left(Q_{i}\right)$ defines the simulated structure factor of the molecular structure after current trial move at the $\mathrm{i}^{\text {th }}$ given momentum transfer $\mathrm{Q}_{i}$, and $N$ is the total number of momentum transfer. 
Since the purpose of RMC is to construct the adsorbent molecular structure with the knowledge of structure factor that is calculated from X-ray diffraction data of a reference structure, it is necessary to accept trial moves that narrow the gap of characteristic indicator between current and reference structure. The Boolean flag "IsMoveAccepted" for accepting or rejecting the trial move based on the chi-square value of previous and current move is given by,

$$
\text { IsMoveAccepted }=\left\{\begin{array}{lr}
\text { True, } & \chi_{S, \text { new }}^{2}<\chi_{S, \text { old }}^{2} \\
\text { True, } & \chi_{S, \text { new }}^{2}>\chi_{S, \text { old }}^{2} \text { and } e^{-\left(\chi_{S, \text { new }}^{2}-\chi_{S, \text { old }}^{2}\right) / 2}>\chi_{D \text { D }, 0.05}^{2} \\
\text { False }, & \text { else }
\end{array}\right.
$$

By randomly moving one atom, a new $\chi_{S}^{2}$ is calculated, and the move is accepted when $\chi_{S \text {, new }}^{2}<$ $\chi_{S, \text { old }}^{2}$ or with a probability of $e^{-\left(\chi_{S, \text { new }}^{2}-\chi_{S, \text { old }}^{2}\right) / 2}$ when $\chi_{S, \text { new }}^{2}>\chi_{S, \text { old }}^{2}$.

The total number of stochastic selection and movement of atoms should be as many as possible to ensure that the difference of characteristic indicator between the current and the reference structures is minimised. Meanwhile, the constraint of minimum atom-atom distance is applied to the RMC simulation to reject the move that leads $\mathrm{Si}-\mathrm{Si}, \mathrm{Si}-\mathrm{O}$ and $\mathrm{O}-\mathrm{O}$ distance to be smaller than the minimum acceptable distance. The minimum atom-atom distances are furnished in Table 3.2

Table 3.2 Minimum atom-atom distance used in the RMC simulation

\begin{tabular}{lccc}
\hline Pair & $\mathrm{Si}-\mathrm{Si}$ & $\mathrm{Si}-0$ & $0-0$ \\
\hline Distance $(\AA)$ & 2.8 & 1.4 & 2.3 \\
\hline
\end{tabular}

Employing experimentally measured X-ray diffraction data [109] with the reverse Monte Carlo (RMC) simulation technique, the amorphous silica structure is formed. For simplicity, the RMC simulation comprises $1000 \mathrm{Si}$ and $2000 \mathrm{O}$ atoms [110]. 
In order to verify the molecular model of silica gel from RMC in the literature [110], a set of crystallographic properties such as bond angle distribution function, radial distribution function and structure factor of the amorphous silica structure are calculated. These results are compared with experimentally measured data.

The total radial distribution in Figure 3.4(a) agrees well with partial radial distribution in Figure 3.4(b), where the first peak occurs at around $1.6 \AA$ and the second peak $2.4 \AA$. While the Si-O bond length in silicate is found between $1.60 \AA$ and $1.64 \AA$ [87], the second peak reflects the correlation between oxygen atoms on the vertices of those tetrahedral [110].

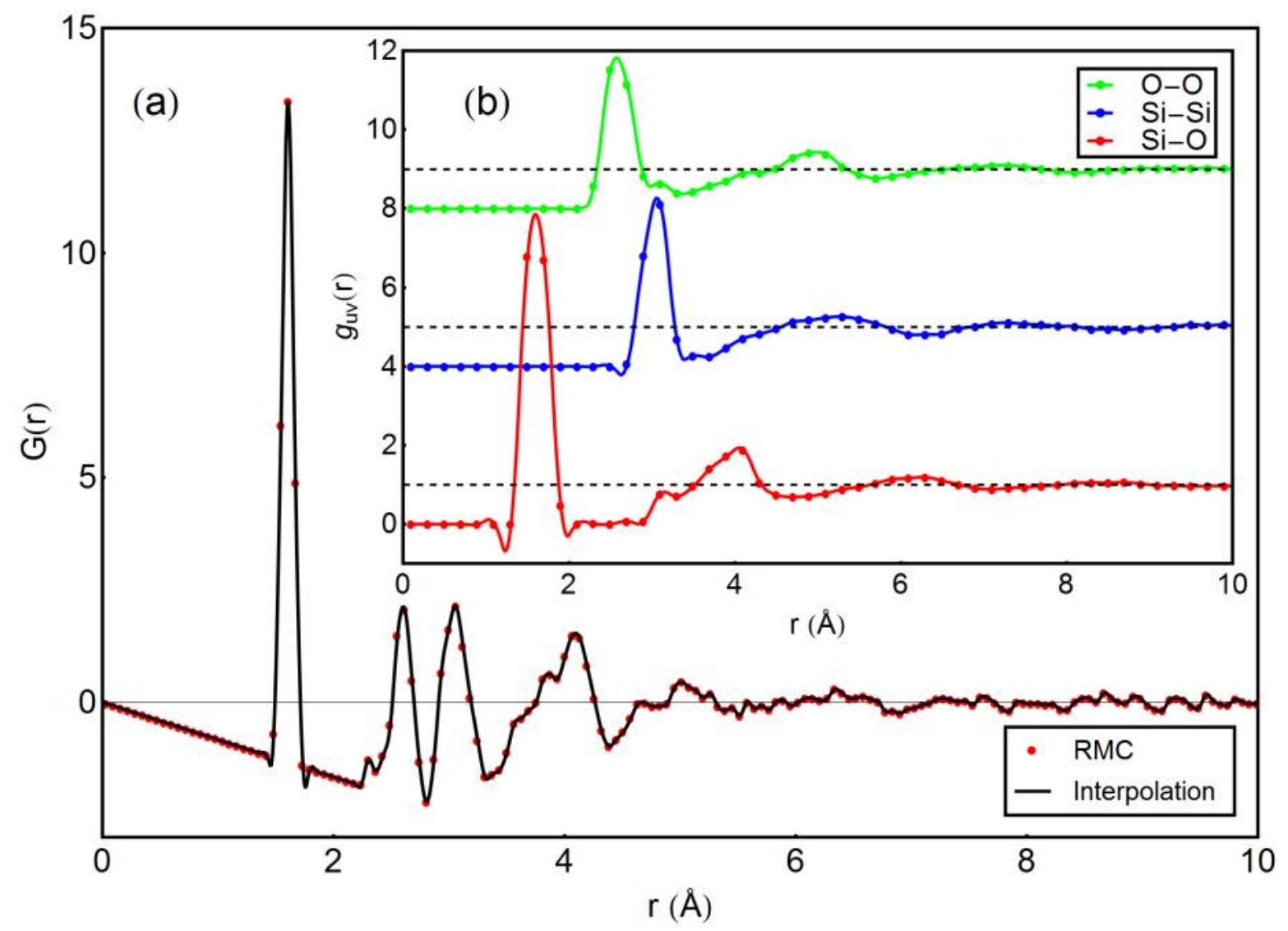

Figure 3.4 Radial distribution function of the $\mathrm{SiO}_{2}$ structure: (a) total radial distribution (b) partial radial distribution of $\mathrm{Si}-\mathrm{Si}, \mathrm{Si}-\mathrm{O}$ and $\mathrm{O}-\mathrm{O}$ 
The bond angle (O-Si-O and $\mathrm{Si}-\mathrm{O}-\mathrm{Si}$ ) distribution function of the silica structure is shown in Figure 3.5 The histogram data is obtained by the sampling angle distribution of the amorphous silica structure with a bin size of $1^{\circ}$ between $75^{\circ} \sim 150^{\circ}$ for $\mathrm{O}-\mathrm{Si}-\mathrm{O}$ and $105^{\circ} \sim 180^{\circ}$ for $\mathrm{Si}-\mathrm{O}-\mathrm{Si}$. Meanwhile, the probability distribution function (PDF) of the bond angle results is also given as a smoothed function of the histogram. The O-Si-O and Si-O-Si are mainly distributed around the two peaks around $104^{\circ} \sim 112^{\circ}$ and $130^{\circ} \sim 145^{\circ}$. These results agree well with available simulation results in the literature [111]. The most probable bond angle (MPBA) of Si-O-Si is found to be $146^{\circ}$ and that of $\mathrm{O}-\mathrm{Si}-\mathrm{O}$ is obtained to be $109.4 \circ$ [112].

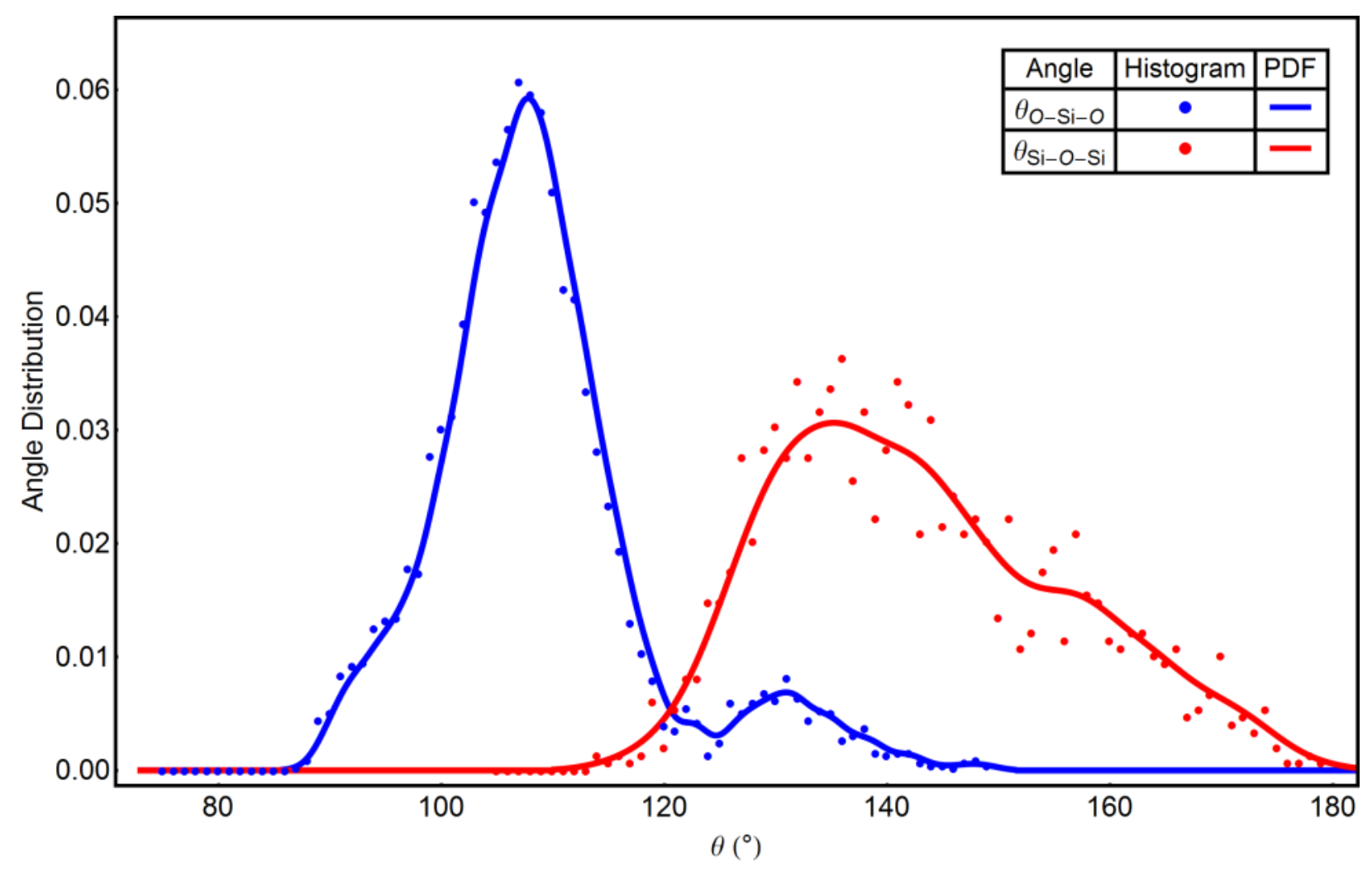

Figure 3.5 Comparison of O-Si-O and $\mathrm{Si}-\mathrm{O}-\mathrm{Si}$ angle distribution function of the $\mathrm{SiO}_{2}$ structure

The total structure factor of the amorphous silica is shown in Figure 3.6 The momentum transfer $Q$ ranges from $0 \sim 20 \AA^{-1}$, for the calculation of structure factor. The calculated $\mathrm{S}(\mathrm{Q})$ results of 
the amorphous silica structure from RMC agree well with adapted $\mathrm{S}(\mathrm{Q})$ data from experimentally measured Q[S(Q)-1]) data by X-Ray Diffraction (XRD) [109].

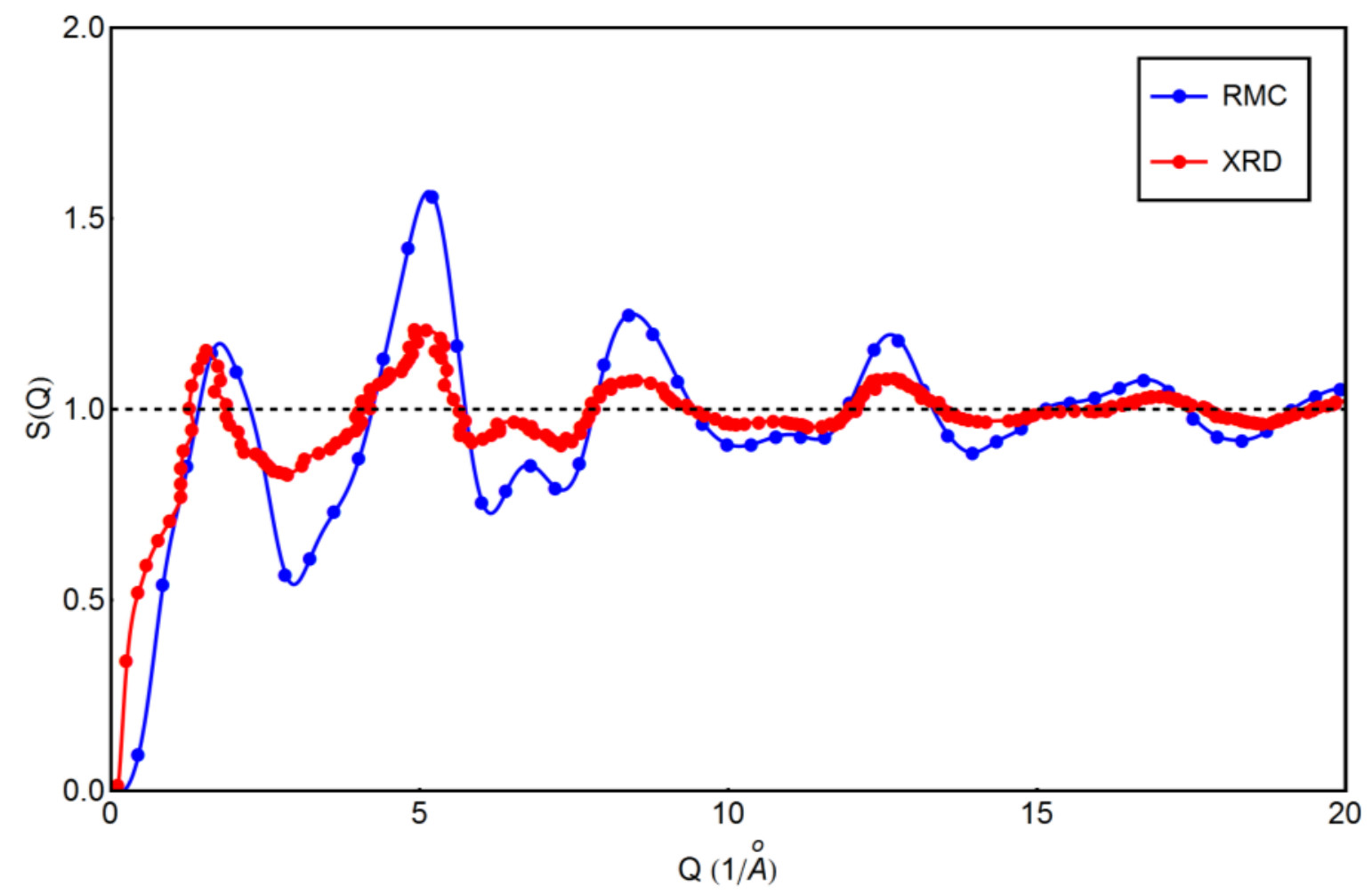

Figure 3.6 Total structure factor of the amorphous silica structure from RMC and XRD

The generated amorphous silica $\left(\mathrm{SiO}_{2}\right)$ structure with $X Y Z$ coordinate system is shown in Figure 3.7, where a water molecule is observed with rotational angles of $\alpha, \beta$ and $\gamma$ correspondingly around three axes of the $X^{\prime} Y^{\prime} Z^{\prime}$ system is placed. The position vectors of both water and $\mathrm{SiO}_{2}$ molecules are described conveniently by coordinates of $x, y$ and $z$, and transfer matrix with translation and rotation. 


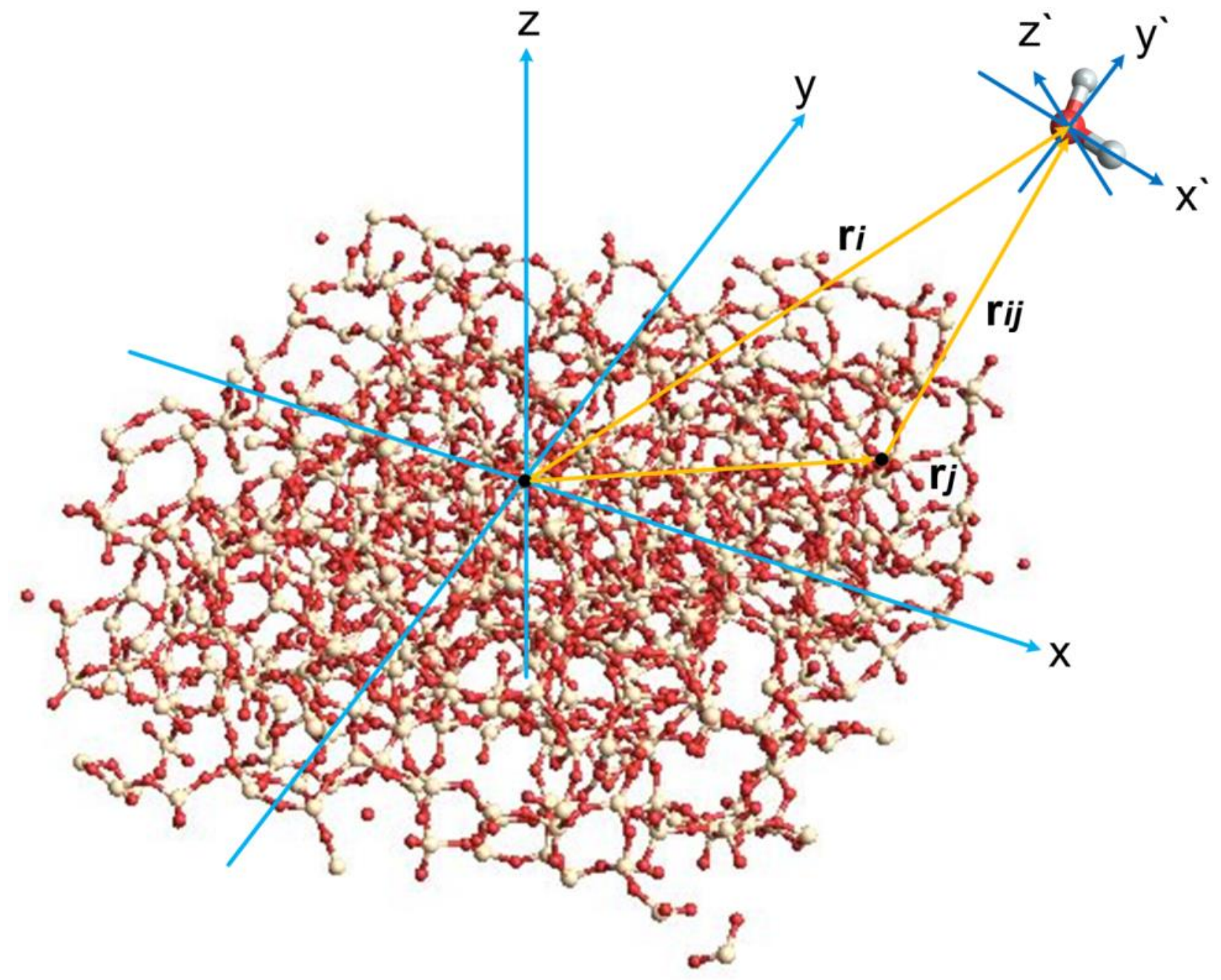

Figure 3.7 The simulation model of silica gel and water molecule. The edge length of the simulation box is $\mathrm{a}=\mathrm{b}=\mathrm{c}=35.7 \AA$. The position vector $\left(\boldsymbol{r}_{i j}=\boldsymbol{r}_{i}-\boldsymbol{r}_{j}\right)$ points from the $\mathrm{j}^{\text {th }}$ atom $(\mathrm{Si}$ or $\mathrm{O})$ of adsorbent to the $\mathrm{i}^{\text {th }}$ atom $(\mathrm{H}$ or $\mathrm{O})$ of the water molecule, where $\boldsymbol{r}_{\boldsymbol{i}}$ is the position vector of $i^{\text {th }}$ atom of water molecule, $\boldsymbol{r}_{\boldsymbol{j}}$ is the position vector of $\mathrm{j}^{\text {th }}$ silicon/oxygen atom. The position vector in water molecule coordinate system $X^{\prime} Y^{\prime} Z^{\prime}$ can be converted to the silica coordinate system $X Y Z$ with a specific rotational matrix and translational vector.

The mathematical modelling for the calculation of the interaction potential and $q_{s t}{ }^{o}$ of silicawater system is shown in Figure 3.8. In this model, the water molecules are placed between two 
layers of $\mathrm{SiO}_{2}$ molecules for simplicity. Here the position vector $\left(\boldsymbol{r}_{\boldsymbol{i}}-\boldsymbol{r}_{\boldsymbol{j}}\right)$ of water molecule can vary freely above the top surface of the silica simulated box. The external normal component value $\mathrm{z}$, which is the vertical distance of water molecule to the silica surface, is related to the total interaction potential between water molecule and two surfaces of silica with the maximum pore width of $\mathrm{H}$. The distance between two silica layers is considered as the pore width for simplicity.

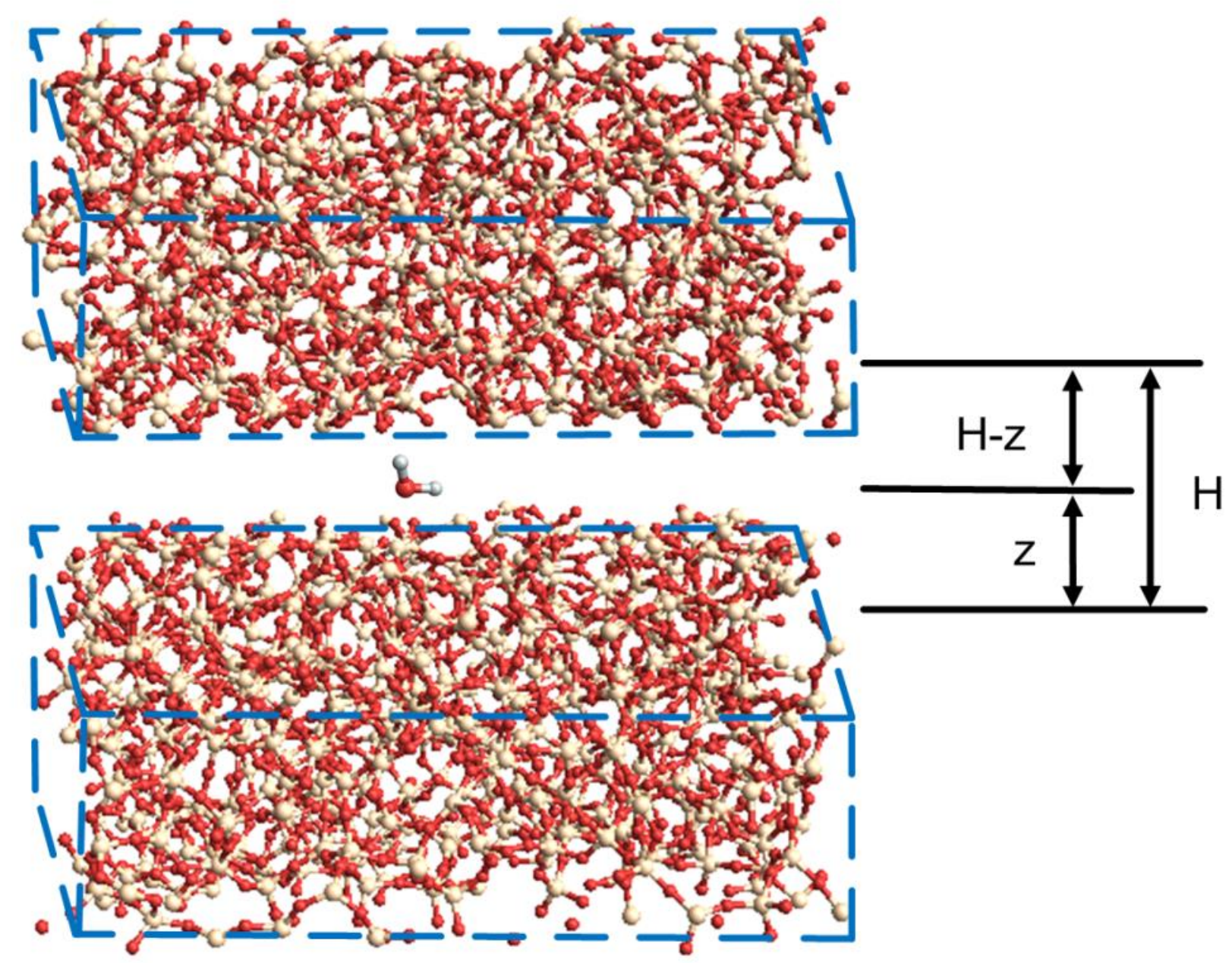

Figure 3.8 The model for calculating the average external wall potential as a function of $z$ for $\mathrm{SiO}_{2}$-Water system. The distance of the centre of water molecule to the surface of silica and the 
opposing wall are $z$ and $H-z$, respectively given the pore width of $H$. The external wall potential of the adsorbate molecule in the pore is the sum of interaction potential with both walls.

Here the water molecule can move freely, which means $\boldsymbol{r}_{\boldsymbol{i}}$ is free. For each $\mathrm{Si}$ or $\mathrm{O}$ atom of $\mathrm{SiO}_{2}$, the position $\boldsymbol{r}_{\boldsymbol{j}}$ is defined in the generated amorphous molecular structure by RMC and the pair Lennard-Jones and electrostatic potentials with water molecule are functions of water-Si/O relative position vector $\boldsymbol{r}_{\boldsymbol{i} j}$.

\section{Interaction Potential of Amorphous $\mathrm{SiO}_{2}$ Surface and Adsorbate Molecules}

For silica and water system, the interaction potential model is obtained by [113],

$$
\mathrm{U}=\mathrm{U}_{\mathrm{LJ}}+\mathrm{U}_{\mathrm{E}}
$$

where $\mathrm{U}_{\mathrm{LJ}}$ is the Lennard-Jones potential and $\mathrm{U}_{\mathrm{E}}$ is the electrostatic potential. The Lennard-Jones potential $\mathrm{U}_{\mathrm{LJ}}$ is determined by the $12-6$ potential

$$
\mathrm{U}_{\mathrm{LJ}}\left(\boldsymbol{r}_{\boldsymbol{i}}-\boldsymbol{r}_{\boldsymbol{j}}\right)=4 \varepsilon_{i j}\left\{\left(\frac{\sigma_{i j}}{\left|\boldsymbol{r}_{\boldsymbol{i}}-\boldsymbol{r}_{j}\right|}\right)^{12}-\left(\frac{\sigma_{i j}}{\left|\boldsymbol{r}_{\boldsymbol{i}}-\boldsymbol{r}_{j}\right|}\right)^{6}\right\}
$$

where $\boldsymbol{r}_{\boldsymbol{i}}$ is the position vector of the $\mathrm{i}^{\text {th }}$ atom of water molecule, $\boldsymbol{r}_{\boldsymbol{j}}$ is the position vector of the $\mathrm{j}^{\text {th }}$ atom of $\mathrm{SiO}_{2}, \varepsilon_{i j}$ and $\sigma_{i j}$ are pair well depth potential and collision diameter. According to the Lorentz-Berthelot combining rules, $\varepsilon_{i j}=\sqrt{\varepsilon_{i} \varepsilon_{j}}$ and $\sigma_{i j}=\frac{\sigma_{i}+\sigma_{j}}{2}$.

The model of electrostatic interaction between silica and water is shown in Figure 3.9. For simplicity, we ignore the bonded effect such as the interaction between the water molecule with functional groups such as hydroxyl group on the surface of silica. The electrostatic interaction between water and $\mathrm{SiO}_{2}$ comprises equal and opposite charges $\left(\mathrm{q}_{\mathrm{H}}\right.$ for water and $\mathrm{q}_{\mathrm{Si}}$, $\mathrm{q}_{\mathrm{O}}$ for $\mathrm{SiO}_{2}$ molecule) with the separation of $\boldsymbol{r}_{i j}$ 


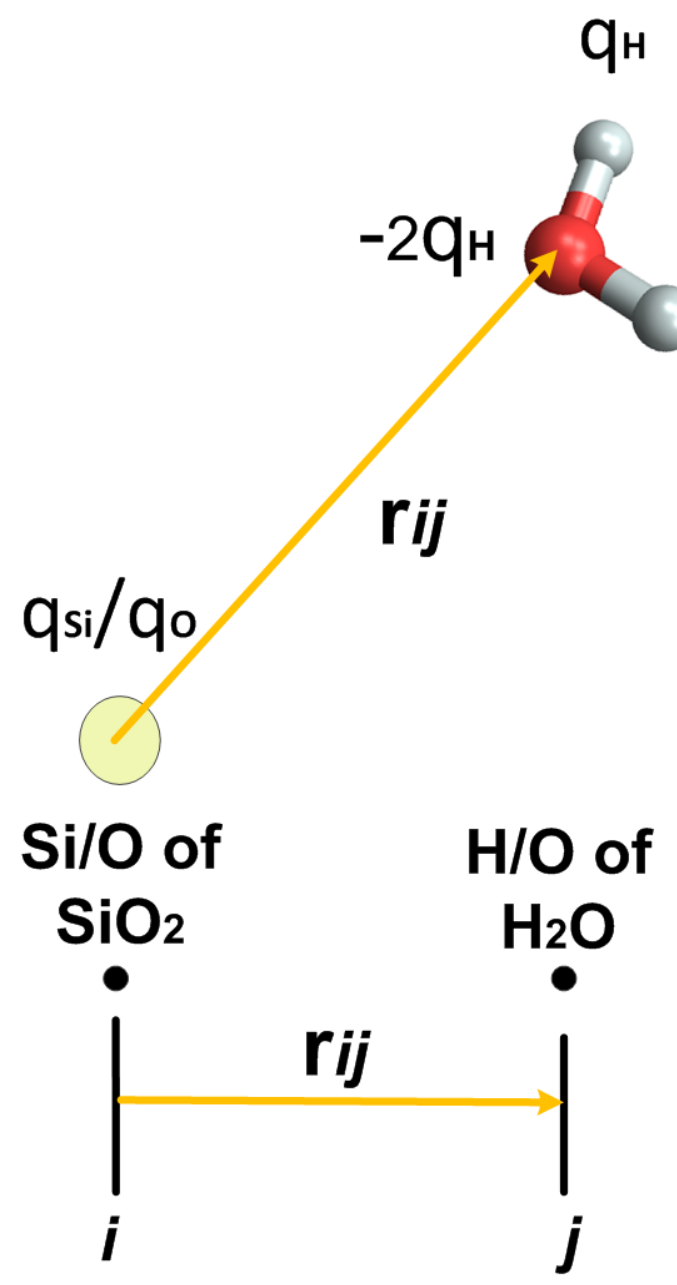

Figure 3.9 Electrostatic interaction between $\mathrm{SiO}_{2}$ and $\mathrm{H}_{2} \mathrm{O}$ molecules. The $i^{\text {th }}$ atom of $\mathrm{SiO}_{2}$ molecule with the electrical charge of $\mathrm{q}_{\mathrm{Si}}$ or $\mathrm{qo}_{\mathrm{O}}$ interacts with the $j^{\text {th }}$ atom of $\mathrm{H}_{2} \mathrm{O}$ molecule with the electrical charge of $q_{H}$ or $q_{O}$. The position vector $\boldsymbol{r}_{i j}$ characterises the distance between the interaction pair.

The electrostatic potential $U_{E}$ of charge-charge interaction is given by

$$
U_{E}\left(\boldsymbol{r}_{\boldsymbol{i}}-\boldsymbol{r}_{\boldsymbol{j}}\right)=\frac{q_{i} q_{j}}{4 \pi \varepsilon_{0}\left|\boldsymbol{r}_{\boldsymbol{i}}-\boldsymbol{r}_{\boldsymbol{j}}\right|}
$$

where $q_{i}$ is the charge of $\mathrm{i}^{\text {th }}$ atom of the adsorbate molecule, $q_{j}$ is the charge of the $\mathrm{j}^{\text {th }}$ atom of slica, $\varepsilon_{0}$ is the vacuum permittivity and $r$ is the charge-charge distance. 
Hence, the total interaction potential depends on the orientation and relative position of the water molecule. Furthermore, with fixed orientation, $x$ and $y$, the total interaction potential which depends only on $\mathrm{z}$ is given by,

$$
\mathrm{U}=\sum_{i=1, j=1}^{A, B} \mathrm{U}_{\mathrm{LJ}}\left(\boldsymbol{r}_{\boldsymbol{i}}-\boldsymbol{r}_{\boldsymbol{j}}\right)+\mathrm{U}_{\mathrm{E}}\left(\boldsymbol{r}_{\boldsymbol{i}}-\boldsymbol{r}_{\boldsymbol{j}}\right)=\sum_{i=1, j=1}^{A, B}\left[\mathrm{U}_{\mathrm{LJ}}(\mathrm{z})+\mathrm{U}_{\mathrm{E}}(\mathrm{z})\right]_{\alpha_{0}, \beta_{0}, \gamma_{0}, x_{0}, y_{0}}=\mathrm{U}(\mathrm{z})
$$

where $\mathrm{A}$ and $\mathrm{B}$ are the total number of atoms in water and $\mathrm{SiO}_{2}$ molecules, $\alpha_{0}, \beta_{0}, \gamma_{0}, x_{0}$ and $y_{0}$ are the fixed rotational angles and positions.

Similar to Equation (3.4), the total interaction potential $V_{\text {ext }}$ is the summation of water molecule interactions with both adsorbent walls with the distance of $\mathrm{z}$ and $\mathrm{H}-\mathrm{z}$, which is expressed by the following equation $[13,114]$

$$
\mathrm{V}_{\mathrm{ext}}(\mathrm{z})=\mathrm{U}(\mathrm{z})+\mathrm{U}(\mathrm{H}-\mathrm{z})
$$

\subsubsection{Molecular Model of Framework Structure and Water Molecules}

It is well known that (i) AQSOA-Z01 is a ferroaluminophosphate (FAPO-5) based zeotype with AFI structure, (ii) AQSOA-Z05 is an aluminophosphate (APO-5) based zeotype with AFI structure and (iii) AQSOA-Z02 is based on the silicoaluminophosphate (SAPO-34) zeotype with CHA structure[115-117]. The composition of AQSOA-Z01 [60] is $\mathrm{Fe}_{\mathrm{x}} \mathrm{Al}_{\mathrm{y}} \mathrm{P}_{\mathrm{z}} \mathrm{O} 2 \cdot \mathrm{nH} 2 \mathrm{O}, \mathrm{x}=0.02-$ $0.10, \mathrm{y}=0.35-0.5, \mathrm{z}=0.4-0.6, \mathrm{n}=0-1$. The sharp peaks of $\mathrm{X}$-ray diffraction (XRD) indicate good crystallinity of both AQSOA-Z01 and AQSOA-Z02 with chemical formulae of $\mathrm{FeAl}_{2}\left(\mathrm{PO}_{4}\right)_{2} \cdot 8 \mathrm{H}_{2} \mathrm{O}$ and $\mathrm{Al}_{0.56} \mathrm{Si}_{0.02} \mathrm{P}_{0.42} \mathrm{O}_{2}$, respectively [18].

The molecular structure for CHA framework types such as AQSOA-Z02 zeolite is shown in Figure 3.10 (a). On the other hand, the molecular structure for AFI framework types such as AQSOA-Z01/Z05 zeolite is shown in Figure 3.10 (b). 

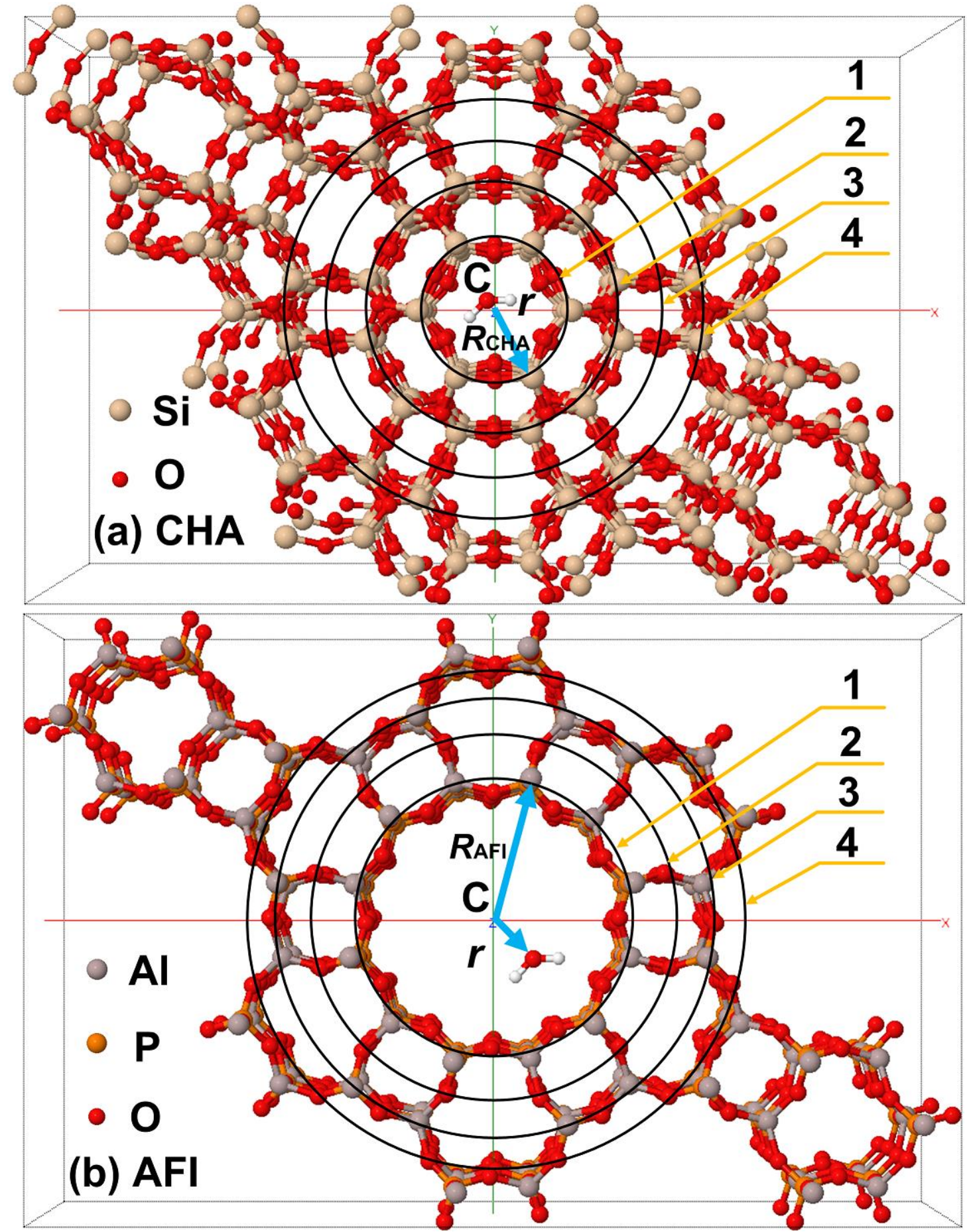

Figure 3.10 The interactions of water molecule with (a) CHA type and (b) AFI type zeolites according to cylindrical pore model. Hence 1, 2, 3 and 4 indicate the layers of cylindrical 
channels; $\mathrm{C}$ is the center of the channels, $\mathrm{r}$ is the distance of one water molecule from the center C.

It is well known that AFI zeolite frameworks such as AQSOA-Z01 and Z05 are ferroaluminophosphate (FAPO-5) and aluminophosphate (APO-5) based zeotypes. Therefore, a molecular structure of $\mathrm{Al}, \mathrm{P}$ and $\mathrm{O}$ atoms is applied to calculate the interaction potentials of water - AFI zeolite systems. On the other hand, the CHA type zeolite such as AQSOA-Z02 silico-alumino-phosphate (SAPO-34) zeotype, and a molecular structure of $\mathrm{Si}$ and $\mathrm{O}$ atoms is applied in the calculation of interaction potentials between water - CHA zeolite system. For modelling and simulation purposes, the location of water molecules adsorbed in CHA and AFI zeolite frameworks are shown in Figure 3.10 (a) and (b). For simplicity, the interactions between water molecule and zeolite framework are calculated by considering multiple layers of cylindrical channels in CHA and AFI zeolite. For CHA zeolite, the radius for four layers of channel is $3.65 \AA, 5.9 \AA, 8.75 \AA$ and $10.88 \AA$. For AFI zeolite (Figure 1b), the radius of four layers of channel is $5.64 \AA, 7.24 \AA, 8.39 \AA$ and $9.69 \AA$. As can be observed from Figure 1 (both CHA and AFI cases), $\mathrm{R}_{\mathrm{CHA}}$ is the radius of the most inner layer (i.e. 1st Layer) of channel and $\mathrm{r}$ is the distance of the water molecule to the center of the channel. This means that the water molecule is closer to the surface of the most inner layer of channel as $r / R_{C H A}$ (i.e. $r / R$ ) increases.

\section{Interaction Potential of Framework Structure and Adsorbate Molecules}

For the zeolite and water system, the 12-6 Lennard-Jones interaction potentials between simulated pairs are applied in the identification favourable adsorptive sites that with larger interaction potential on the surface of zeolites. The sum of pair-wise additive interaction potential is varied with the relative position of water molecule on the surface of zeolite framework and is given by, 


$$
U_{L J}\left(\boldsymbol{r}_{O}\right)=\sum_{i=1}^{N} 4 \varepsilon_{i, O}\left[\left(\frac{\sigma_{i, O}}{\left|\boldsymbol{r}_{O}-\boldsymbol{r}_{i}\right|}\right)^{12}-\left(\frac{\sigma_{i, O}}{\left|\boldsymbol{r}_{O}-\boldsymbol{r}_{i}\right|}\right)^{6}\right]
$$

where $\boldsymbol{r}_{O}$ is the position vector of oxygen atom of water molecule, $\boldsymbol{r}_{i}$ is the position vector of $i$ th atom of zeolite, $N$ is the number of atoms of zeolite, $\varepsilon_{i, O}$ is the well depth potential of the atom of zeolite with oxygen atom of water molecule and $\sigma_{i, O}$ is the collision diameter.

By varying the position of the water molecule on the surface of zeolite $\left(\boldsymbol{r}_{O}\right)$, the contour plot of potential with fixed $\mathrm{z}$ distance and variable $(\mathrm{x}, \mathrm{y})$ distance of water molecule is used to identify the favorable adsorptive sites.

The interaction potential of water molecule with a single layer cylindrical channel with radius of $R$ in zeolite framework is given by [118-120],

$$
\mathrm{w}(\mathrm{r})=\frac{5}{2} \pi \varepsilon_{1 s}\left\{\frac{21}{32}\left(\frac{\sigma_{12}}{R}\right)^{10}\left[\sum_{k=0}^{\infty} \alpha_{k}\left(\frac{r}{R}\right)^{2 k}\right]-\left(\frac{\sigma_{12}}{R}\right)^{4}\left[\sum_{k=0}^{\infty} \beta_{k}\left(\frac{r}{R}\right)^{2 k}\right]\right\}
$$

where $\mathrm{r}$ is the radial distance of water molecule to the centre of the channel. The coefficients $\alpha_{k}$ and $\beta_{k}$ are defined by,

$$
\begin{aligned}
& \alpha_{k}=\left(\frac{\boldsymbol{\Gamma}(-4.5)}{\boldsymbol{\Gamma}(-4.5-k) \boldsymbol{\Gamma}(k+1)}\right)^{2} \\
& \beta_{k}=\left(\frac{\boldsymbol{\Gamma}(-1.5)}{\boldsymbol{\Gamma}(-1.5-k) \boldsymbol{\Gamma}(k+1)}\right)^{2}
\end{aligned}
$$

The gas-solid potential parameter $\varepsilon_{1 s}$ is given by,

$$
\varepsilon_{1 s}=\frac{6}{5} \pi \rho_{1 s} \varepsilon_{12} \sigma_{12}^{2}
$$

where $\rho_{1 s}$ is the atom number density at the surface of cylindrical channel, $\varepsilon_{12}$ is the well depth potential of interaction pair and $\sigma_{12}$ is the collision diameter. 
The well depth potentials and collision diameters used in the simulation are shown in Table 3.3. Only the interaction potential between oxygen atom of water molecule and atoms of zeolite framework is considered. These Lennard Jones parameters are from the literature [121-124] while the Lorentz-Berthelot combining rules, $\sigma_{i j}=\frac{\sigma_{i}+\sigma_{j}}{2}$ and $\varepsilon_{i j}=\sqrt{\varepsilon_{i} \varepsilon_{j}}$ are used in the simulation.

Table 3.3 Well depth potentials and collision diameters of simulated pairs

\begin{tabular}{ccccc}
\hline Zeolite Type & Atom $\boldsymbol{i}$ & Atom $\boldsymbol{j}$ & $\boldsymbol{\sigma}_{\boldsymbol{i} \boldsymbol{j}}(\AA)$ & $\boldsymbol{\varepsilon}_{\boldsymbol{i} \boldsymbol{j}} / \boldsymbol{k}_{\boldsymbol{B}}(\mathrm{K})$ \\
\hline $\mathrm{CHA}\left(\mathrm{SiO}_{2}\right)$ & $\mathrm{Si}$ & $\mathrm{O}$ & 3.49 & 125.79 \\
& $\mathrm{O}$ & $\mathrm{O}$ & 3.14 & 48.59 \\
\hline $\mathrm{AFI}\left(\mathrm{AlPO}_{4}\right)$ & $\mathrm{P}$ & $\mathrm{O}$ & 3.58 & 80.54 \\
& $\mathrm{O}$ & $\mathrm{O}$ & 3.14 & 48.59 \\
\hline
\end{tabular}

Another type of interaction potential of water molecule with zeolite framework is described by [105],

$$
U_{\mathrm{LJ}}(\mathrm{z})=2 \varepsilon_{s f} \rho_{s} \sigma_{s f}^{2} \Delta\left[\frac{2}{5}\left(\frac{\sigma_{s f}}{z}\right)^{10}-\left(\frac{\sigma_{s f}}{z}\right)^{4}-\frac{\sigma_{s f}^{4}}{3 \Delta(z+0.61 \Delta)^{3}}\right]
$$

where $\sigma_{\mathrm{sf}}$ and $\varepsilon_{\mathrm{sf}}$ are the solid-fluid collision diameter and the solid-fluid well depth potential, respectively. $\Delta$ is the separation between the atom planes and $\rho_{\mathrm{s}}$ is the density of the solid. For simplicity, the LJ potential is considered as pair-wise additive and the sum of all the interaction pairs is the total LJ interaction potential.

\subsection{Isosteric Heat of Adsorption at Zero Coverage}


The average isosteric heat of adsorption is determined on the basis of both the potential function of pore width and the pore structure. Thermodynamic methods to calculate the average isosteric heat of adsorption for both parallel slit and cylindrical shape pores are presented in the following sections.

\subsubsection{Isosteric Heat of Adsorption for Parallel Slit Pore}

For graphite and polar molecules together with silica and water system, $q_{s t}^{o}$ is calculated using a parallel slit pore model and therefore the interaction of adsorbate molecule with the adsorbent pore is considered as the sum of the interaction of one adsorbate molecule with two opposing surfaces consisting of multi-layer of adsorbent atoms.

Employing the development of Steel [105], the isosteric heat of adsorption at zero surface coverage is $q_{s t}^{o}=-\left.R T^{2} \frac{\partial \ln K_{H}}{\partial T}\right|_{x}$, where $x$ is the amount of adsorbate uptake, $K_{H}$ is the Henry's law constant and $R$ indicates the ideal gas constant. The Henry's coefficient is given by $K_{H}=\frac{1}{A}\left(\frac{Z_{1}}{k T}\right)$, where $\mathrm{A}$ is the adsorbent surface area and $Z_{1}=\int_{V} \exp \left[-V_{e x t}(z) / k T\right] d z . \mathrm{V}_{\text {ext }}$ is the total external wall potential and is calculated by equation 3.4. Substituting the values of $K_{H}$ and $Z_{l}$ in the $q_{s t}^{o}$ equation, the average isosteric heat of adsorption as a function of pore width, $\mathrm{H}$, can be calculated as $[105,125]$,

$$
q_{s t}^{\circ}(\mathrm{H})=\mathrm{RT}-\mathrm{N}_{\mathrm{A}} \bar{V}_{e x t}(z)=\mathrm{RT}-\mathrm{N}_{\mathrm{A}} \frac{\int_{0}^{\mathrm{H}} \mathrm{V}_{\text {ext }}(\mathrm{z}) \mathrm{e}^{\left(-\frac{\mathrm{V}_{\text {ext }}(\mathrm{z})}{k_{B} \mathrm{~T}}\right)} \mathrm{dz}}{\int_{0}^{\mathrm{H}} \mathrm{e}^{\left(-\frac{\mathrm{V}_{\text {ext }}(\mathrm{z})}{k_{B} \mathrm{~T}}\right)} \mathrm{dz}}
$$

Here $\mathrm{R}$ is the ideal gas constant, $\mathrm{T}$ is the adsorption temperature, $\mathrm{N}_{\mathrm{A}}$ is the Avogadro number and $k_{B}$ is the Boltzmann constant. 
$q_{s t}^{o}$ is the separate contributions from (i) thermal energy, and (ii) adsorbate - graphite basal plane/amorphous silica surface interactions. Hence the adsorbate-adsorbate interactions are not considered due to low densities of adsorbate molecules in the Henry's region. It should be noted here that with the fixed distance, $z$, varying from adsorbate molecule to honeycomb carbon lattice, the total interaction potential depends on the relative position, $\mathrm{X}$ and $\mathrm{Y}$, of adsorbate molecule to the hexagonal unit cell of graphite as shown in Figure 3.1.

\subsubsection{Isosteric Heat of Adsorption for Cylindrical Pore}

For zeolite and water system, the Boltzmann weighted average potential is given by

$$
\bar{w}=\frac{\int_{0}^{R}\left(w(r) e^{-\frac{w(r)}{k_{B} T}}\right) r d r}{\int_{0}^{R}\left(e^{-\frac{w(r)}{k_{B} T}}-1\right) r d r}
$$

where $R$ is the radius of the cylindrical pore, $w(r)$ is the interaction potential as a function of distance $r$ of adsorbent molecule to the centre of the cylindrical pore, $k_{B}$ is the Boltzmann constant and $T$ is the temperature.

Therefore, the isosteric heat of adsorption is then given by

$$
q_{s t}{ }^{o}=R T-N_{A} \bar{w}
$$

where $R$ is the universal gas constant and $N_{A}$ is the Avogadro number.

\subsection{Summary}

In this chapter, the molecular and pore models of adsorbates with lattice, amorphous surfaces and framework structure are constructed and described. The interaction potential due to adsorbate adsorbent interactions is defined as a function of pore width. Therefore, the isosteric heat of adsorption of three different types of pore structure is connected to the pore width. This new knowledge can be further used in the performance evaluation of adsorption assisted cooling or 
desalination cycle based systems. Employing the formulations as presented in this chapter, we can calculate the interaction potentials and the isosteric heat at zero coverage for activated carbon $+\mathrm{CO}_{2}$, water, silica gel $\left(\mathrm{SiO}_{2}\right)+$ water and CHA and AFI types zeolites + water systems as these pairs are generally used for cooling and desalination purposes. The simulation results are presented in the next chapter. 


\section{Chapter 4 Simulation Results of Isosteric Heat of Adsorption at}

\section{Zero Coverage}

\subsection{Introduction}

The isosteric heat at zero coverage is due to the contributions from (i) thermal energy, and (ii) adsorbate - adsorbent (e.g. graphite basal plane, amorphous silica, zeolite frameworks etc.) surface interactions. In simulation procedures, the adsorbate-adsorbate interactions are not considered due to low the densities of adsorbate molecules in the Henry's region. It should be noted here that with the distance varying from adsorbate molecule to the adsorbent surface, the total interaction potential depends on the relative position of the adsorbate molecules. For hydrogen and oxygen atoms, the partial charges are 0.4238 and -0.8476 elementary charge. On the other hand, the hydrogen-oxygen-hydrogen angle is $104.5^{\circ}$ and hydrogen-oxygen bond length is $0.95728 \AA$ for water molecule in TIP3P water model [108], which yields both non-zero dipolar and quadrupolar moments. For various adsorbate molecules and carbon atoms, the potential, collision diameter and anisotropy parameters for calculating L-J potential are listed in Table $4.1[42,80,106,126-128]$, and the dipole moment, polarisability of dipole and quadrupole moment for calculating electrostatic potential are listed in Table 4.2 [129-131].

Table 4.1 Simulation parameters for calculating Lennard-Jones potential

\begin{tabular}{|c|c|c|c|c|}
\hline Atom Pair & $\varepsilon(\mathrm{meV})$ & $\sigma(\AA)$ & $\gamma_{A}$ & $\gamma_{R}$ \\
\hline C-H & 2.265 & 2.965 & 0.4 & -0.54 \\
\hline C-C & 2.981 & 3.305 & 0.4 & -1.05 \\
\hline C-N & 2.811 & 3.390 & 0.4 & -1.05 \\
\hline C-O & 3.450 & 3.141 & 0.4 & -1.05 \\
\hline
\end{tabular}


Table 4.2 Simulation parameters for calculating electrostatic potential

\begin{tabular}{|c|c|c|c|c|c|c|c|}
\hline Molecule & $\boldsymbol{\mu}(\mathrm{D})$ & $\boldsymbol{\alpha}_{x x}$ & $\boldsymbol{\alpha}_{y y}$ & $\boldsymbol{\alpha}_{z z}$ & $\boldsymbol{\Theta}_{x x}(\mathrm{D} \AA)$ & $\boldsymbol{\Theta}_{y y}(\mathrm{D} \AA)$ & $\boldsymbol{\Theta}_{z z}(\mathrm{D} \AA)$ \\
\hline Carbon & - & $1.44 \AA^{3}$ & $1.44 \AA^{3}$ & $0.41 \AA^{3}$ & -0.5 & -0.5 & 1 \\
\hline Water & 1.855 & $1.53 \AA^{3}$ & $1.42 \AA^{3}$ & $1.47 \AA^{3}$ & 2.63 & -2.50 & -0.13 \\
\hline Methanol & 1.69 & $3.69 \AA^{3}$ & $3.25 \AA^{3}$ & $3.06 \AA^{3}$ & - & - & - \\
\hline Ethanol & 1.69 & $30.37 \mathrm{Bohr}^{3}$ & $33.61 \mathrm{Bohr}^{3}$ & $38.87 \mathrm{Bohr}^{3}$ & - & - & - \\
\hline
\end{tabular}

*For polarizability, $1 \AA^{3}=1.11265 \times 10^{-40} \mathrm{C} \cdot \mathrm{m}^{2} / \mathrm{V}, 1 \mathrm{Bohr}^{3}=1.648773 \times 10^{-41} \mathrm{C} \cdot \mathrm{m}^{2} / \mathrm{V}$

\subsection{Results of Interaction Potentials}

\subsubsection{Interaction Potential of Graphite with Polar Molecules}

The interactions between the graphite and water are presented with respect to five different orientations of water molecules and these are shown in Figure 4.1. Note that the O-C distances are not important here as Figure 4.1 aims to describe five orientations rather than distance between graphite and the water molecule. In fact, the molecule-graphite distance ( $\mathrm{z}$ ) of the five orientations can be varied from 2 to $10 \AA$ for the calculation of interaction potential.

For water molecule at orientation 1, the oxygen atom is at the origin and one hydrogen atom is at $\mathrm{X}$ axis while the $\mathrm{H}-\mathrm{O}-\mathrm{H}$ is found in $\mathrm{XY}$ plane. The oxygen-graphite distance is $3 \AA$. For water molecule at Orientation 2, the oxygen atom is observed at the origin. Here one of the hydrogen atoms is at $\mathrm{X}$ axis and the other follows toward the graphite surface while the $\mathrm{H}-\mathrm{O}-\mathrm{H}$ plane is found at XZ plane. The oxygen-graphite distance is calculated as $3.5 \AA$. The oxygen atom is at the origin for orientation 3 , where one hydrogen atom is at $\mathrm{X}$ axis, the other hydrogen atom is away from the graphite and the $\mathrm{H}-\mathrm{O}-\mathrm{H}$ orientation is at $\mathrm{XZ}$ plane. The oxygen-graphite distance is found to be $3 \AA$. The orientation 4 of water molecule is also shown in Figure 4.1, where the oxygen atom is at the origin, both of the hydrogen atoms are pointed away from the graphite, and the angle between H-O bond and X axis is $\frac{180^{\circ}-104.52^{\circ}}{2}=37.74^{\circ}$. The H-O-H orientation is 
observed with respect to XZ plane. The oxygen-graphite distance is $3 \AA$. For the water molecule at fifth orientation, the oxygen atom is at the origin. Both of the hydrogens atoms are pointed towards the graphite surface and the angle between $\mathrm{H}-\mathrm{O}$ bond and $\mathrm{X}$ axis is $\frac{180^{\circ}-104.52^{\circ}}{2}=37.74^{\circ}$. The H-O-H plane is parallel to the XZ plane. The oxygen-graphite distance is $3 \AA$.
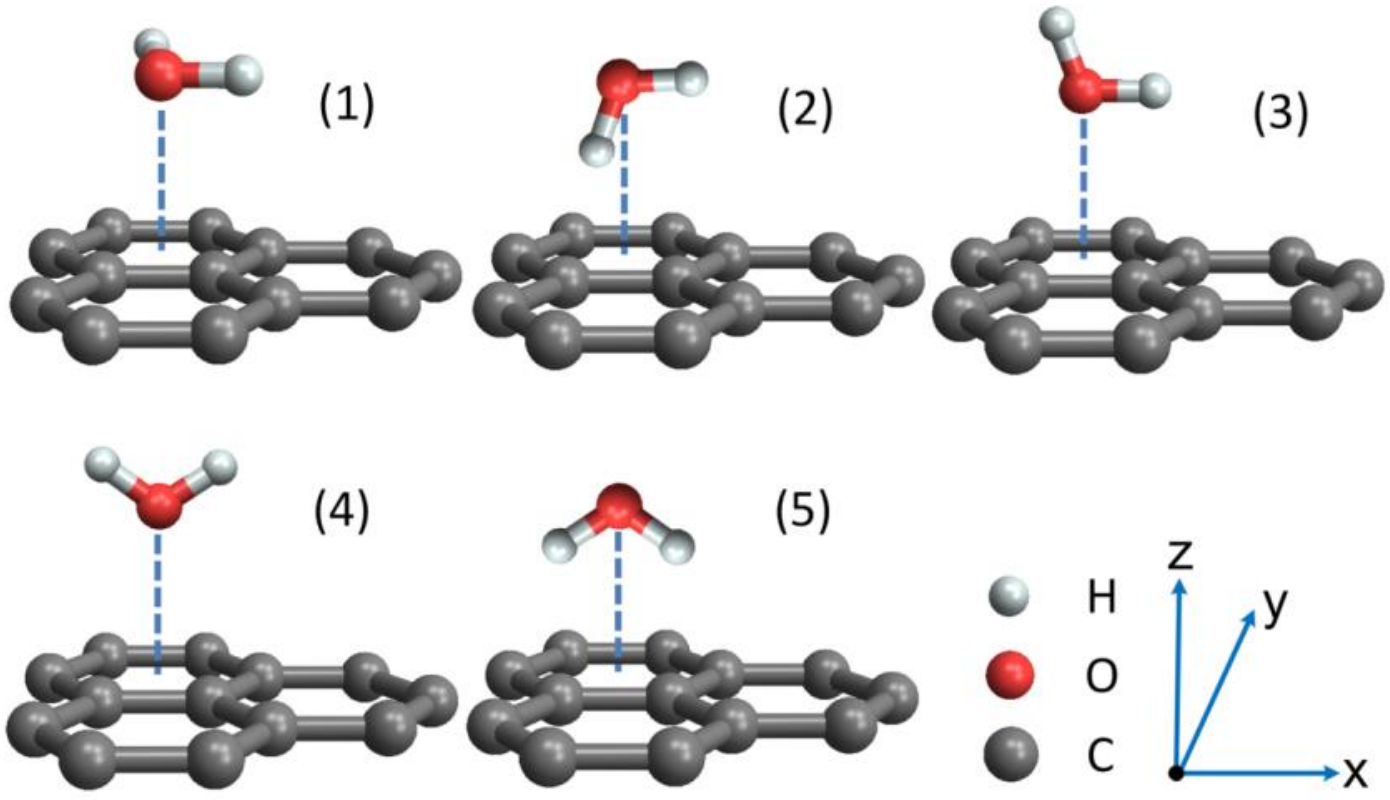

Figure 4.1 Five different orientations of a water molecule on the graphite surface at the distance of $\mathrm{z}$ in $\mathrm{Z}$ direction

The interaction energy of a water molecule with the graphite surface as a function of $\mathrm{O}{ }^{\cdots} \mathrm{C}$ separations (z) for five selected water orientations is shown in Figure 4.2 


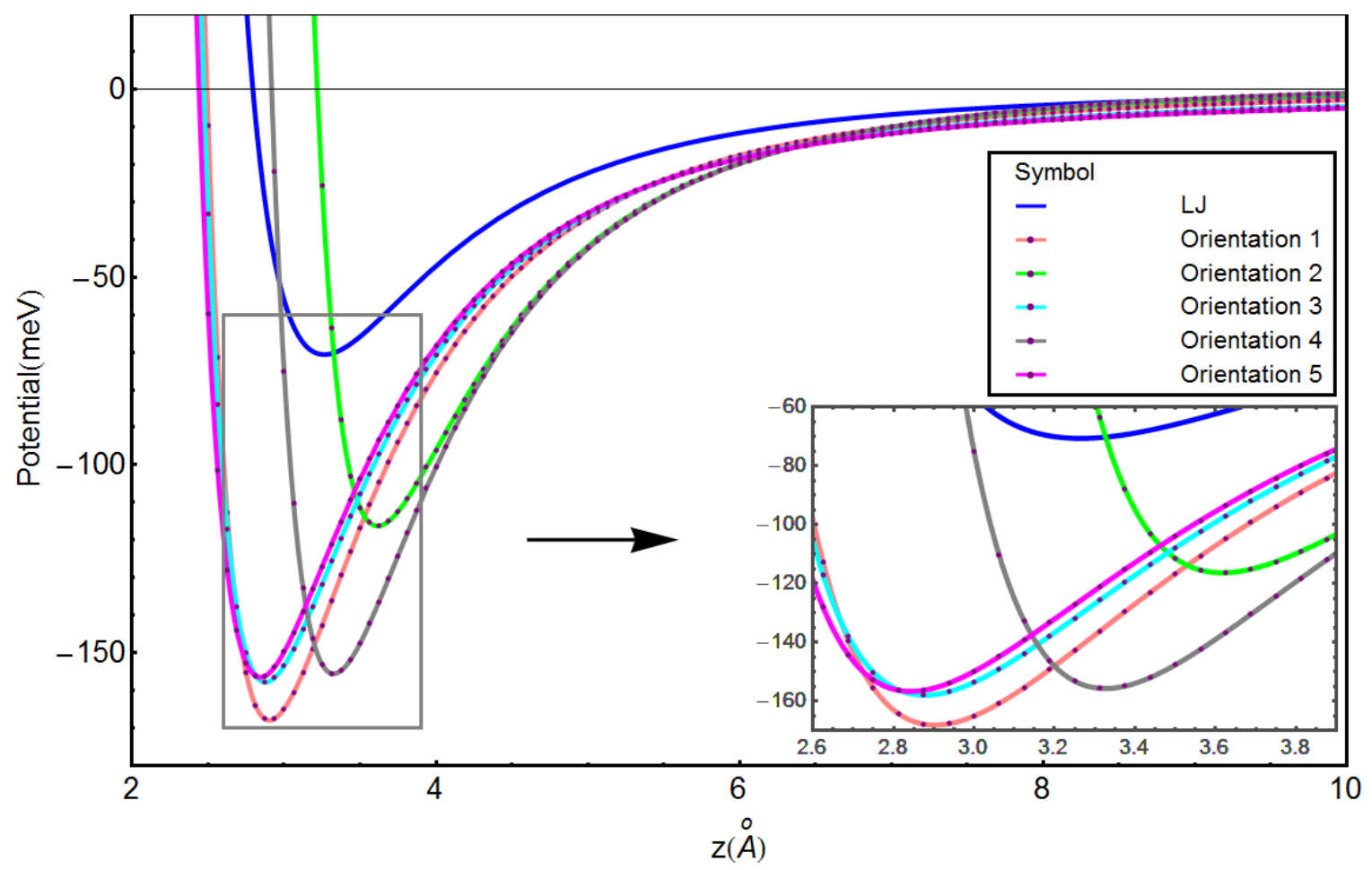

Figure 4.2 Interaction energy of a water molecule with the graphite surface as a function of $\mathrm{C} \cdots \mathrm{O}$ separation (z) for five different orientations.

It is clearly observed that the minimum $\mathrm{H} \cdots \mathrm{C}$ potential is overlapped with the repulsive portion of the $\mathrm{O} \cdots \mathrm{C}$ potential, and this overlap makes the linear $\mathrm{C} \cdots \mathrm{H}-\mathrm{O}$ configuration most favourable. Any deviation from linearity increases the overlap and makes $\mathrm{C} \cdots \mathrm{H}-\mathrm{O}$ configuration more repulsive. It is found that the interaction potentials of orientations $1,3,4$ and 5 are larger than those of orientation 2. From orientations 2 and 5, it is found that one or two hydrogen atoms of water molecule are pointed towards the basal plane of graphite, i.e., $\mathrm{H}$ atoms are closer to carbon atoms at the same $\mathrm{O} \cdots \mathrm{C}$ distance. For also orientations 2 and 5, the smaller interaction potential can be explained by the dramatically increased positive repulsion in Lenard-Jones potential added to the total negative potential as the distance (z) between the pair is decreased. The potential energy with respect to maximum well depth ranges from $-116 \mathrm{meV}$ to $-160 \mathrm{meV}$ depending on the various orientations of water molecule, which agrees well with the available 
experimental value of - $156 \mathrm{meV}[132]$ as well as calculated value $-149 \mathrm{meV}$ reported by Markovic et al. [133] and $-126 \mathrm{meV}$ reported by Lin et al. [134] for water adsorption on graphene at zero surface coverage. It is also found that the electrostatic as well as induction potential increases faster when the separation distance $(\mathrm{z})$ is smaller than $3 \AA$ (for water). The calculated potential energy surfaces for the water molecule with various orientations are explained in the Appendix A.

Figure 4.3 shows three different orientations of one methanol molecule on graphite surface at the beginning of adsorption. Here the carbon and oxygen bond is almost parallel to the carboncarbon bond of graphite or the basal panel (XY plane) as observed in orientation 1 . The $\mathrm{CH}_{3}-\mathrm{OH}$ molecule is rotated $90^{\circ}$ with respect to X-axis (orientation 2). On the other hand, the methanol molecule is rotated $-90^{\circ}$ above the graphite surface (orientation 3 ).
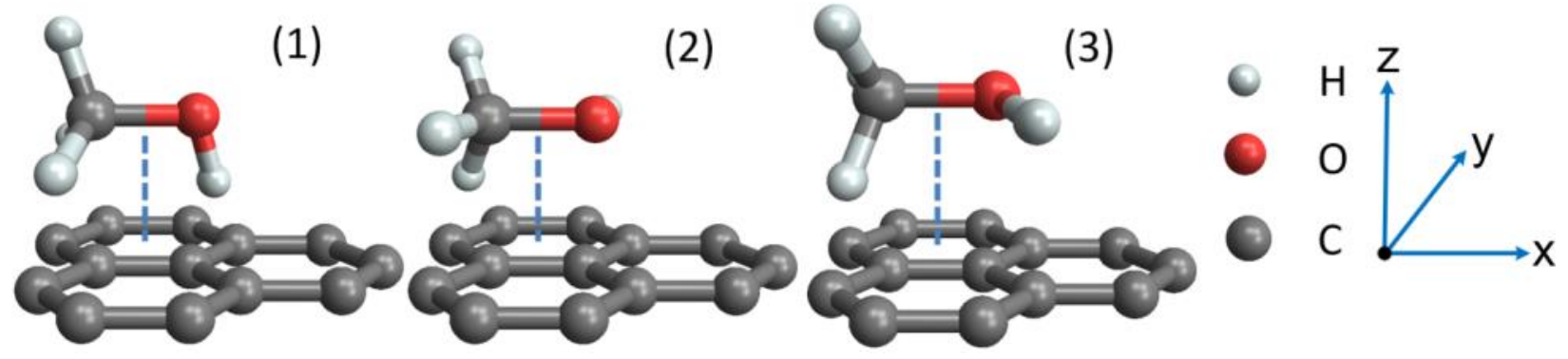

Figure 4.3 Three different orientations of a methanol molecule on the graphite surface at the distance of $\mathrm{z}$ in $\mathrm{Z}$ direction

A plot of binding interactions between one methanol molecule and graphite surface is shown in Figure 4.4 for three different $\mathrm{CH}_{3}-\mathrm{OH}$ orientations. The maximum potential well depth, $\mathrm{U}_{\mathrm{mM}}$, of $\left(\mathrm{CH}_{3}-\mathrm{OH} \cdots \mathrm{C}\right)$ varies from $-210 \mathrm{meV}$ to $-180 \mathrm{meV}$ when the methanol molecule is rotated from 0 to $90^{\circ}$ along the $\mathrm{X}$-axis. On the other hand, the $\mathrm{U}_{\mathrm{mM}}$ of $\left(\mathrm{CH}_{3}-\mathrm{OH} \cdots \mathrm{C}\right)$ ranges from $-210 \mathrm{meV}$ to $170 \mathrm{meV}$ for the rotational angle of methanol varying from 0 to $-90^{\circ}$ along its $\mathrm{X}$-axis. The 
induction potential increases exponentially for the separation distance decreasing from $3 \AA$ to 0 . However the electrostatic potential is very small (close to zero, see Appendix A).

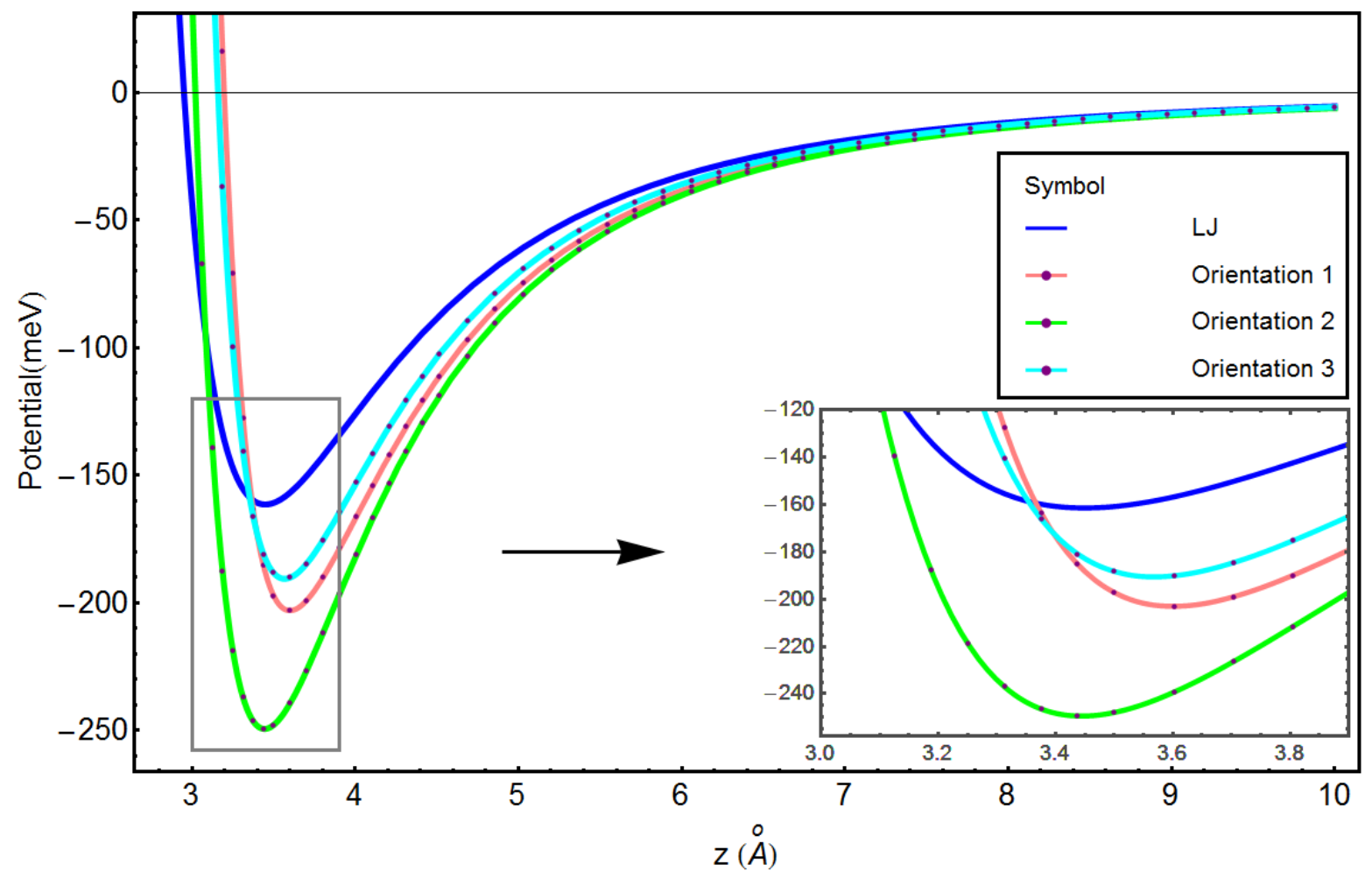

Figure 4.4 Interaction energy of a methanol molecule with the graphite surface as a function of $\mathrm{C} \cdots \mathrm{CH}_{3} \mathrm{OH}$ separation (z) for five different orientations

Figure 4.5 also shows three different orientations of ethanol molecule on a graphite surface. In the orientation 1 of ethanol molecule, both carbon-carbon and carbon-oxygen bonds are almost parallel to the carbon-carbon bond of graphite above the XY plane. From simulation results, it is found that the maximum potential well depth $\mathrm{V}_{\text {ext }}\left(\mathrm{C} \cdots \mathrm{CH}_{3}-\mathrm{CH}_{2}-\mathrm{OH}\right)$ varies from $-300 \mathrm{meV}$ to $270 \mathrm{meV}$ for the rotational angle of ethanol varying from $-90^{\circ}$ to $90^{\circ}$ with respect to its $\mathrm{X}$-axis. 


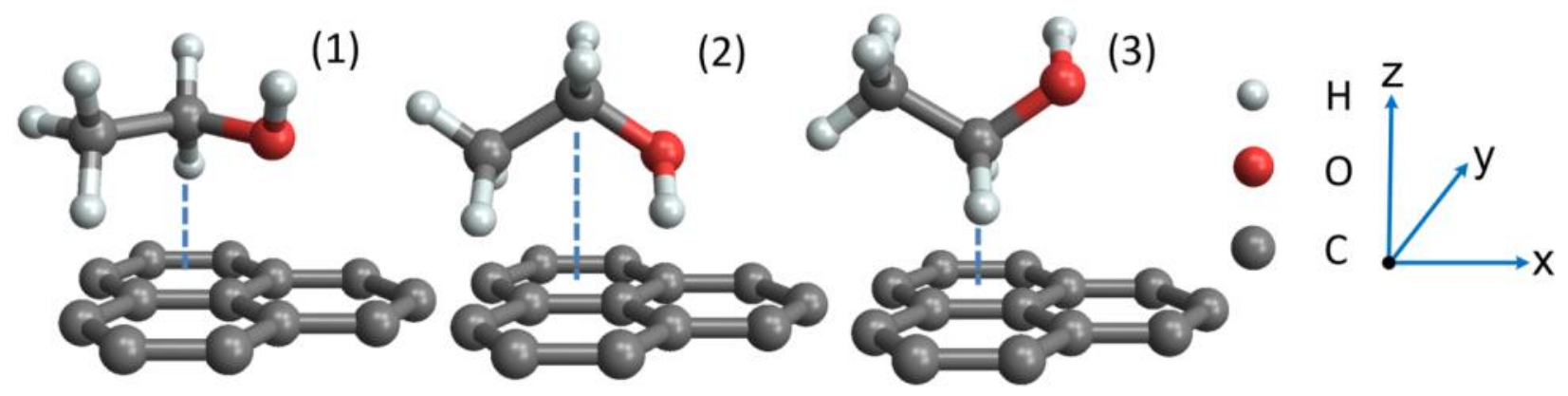

Figure 4.5 Three different orientations of an ethanol molecule on the graphite surface at the distance of $\mathrm{z}$ in $\mathrm{Z}$ direction

The interaction potential as a function of $\mathrm{z}$ is shown in Figure 4.6 It should be noted here that the induction potential increases exponentially for the separation distance (z) decreasing from $3 \AA$ to 0 . However the electrostatic potential remains constant with very small values (close to zero, see Appendix A).

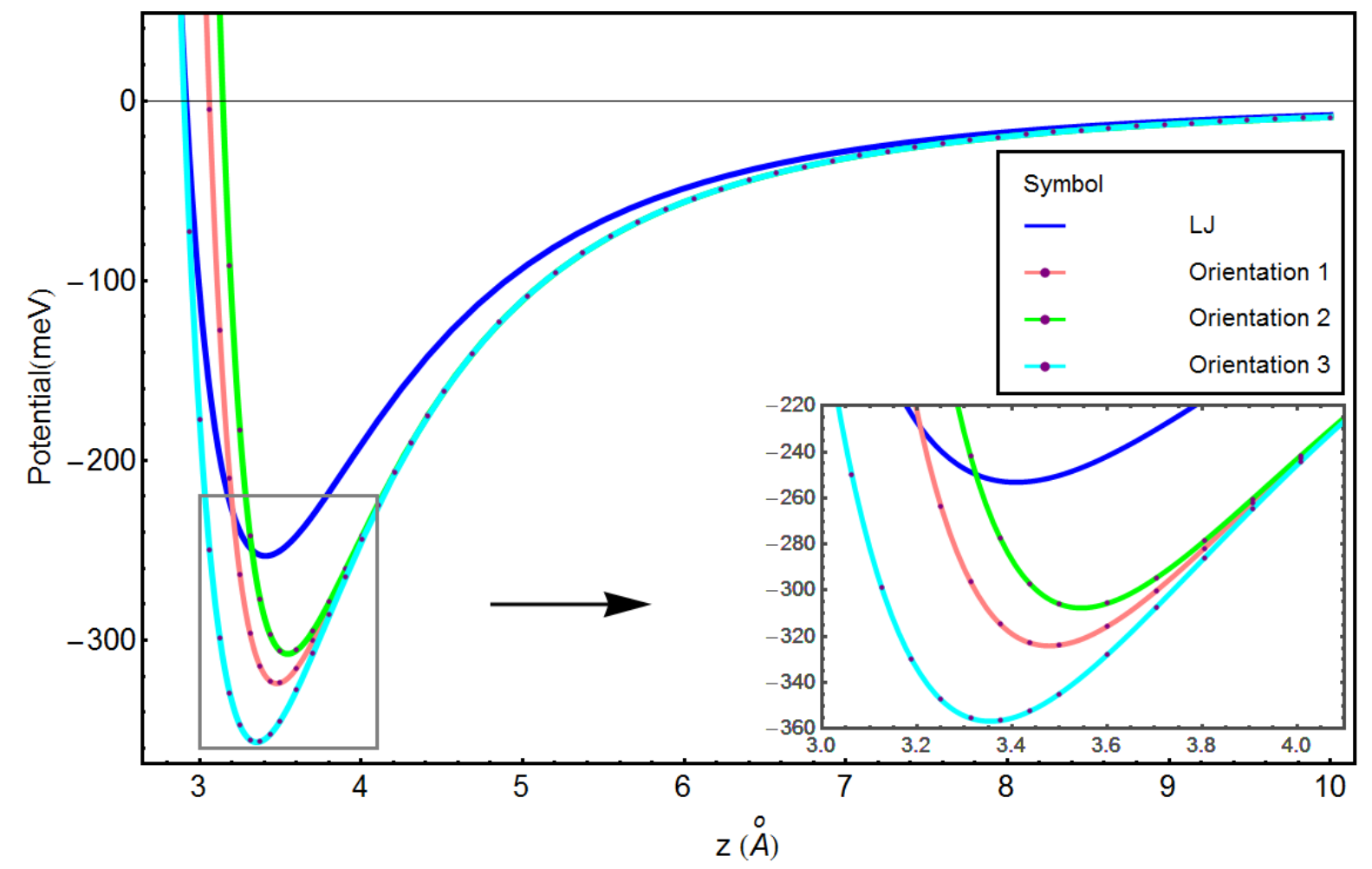

Figure 4.6 Interaction energy of an ethanol molecule with the graphite surface as a function of $\mathrm{C} \cdots \mathrm{CH}_{3} \mathrm{CH}_{2} \mathrm{OH}$ separation (z) for five different orientation 
Figure 4.7 shows the maximum interactions for (a) orientation 1 of water molecule (total -167.75 $\mathrm{meV}=-153.15 \mathrm{meV}$ L-J $-14.61 \mathrm{meV}$ induction $+0.16 \mathrm{meV}$ electrostatic at $2.9 \AA$ ), (b) orientation 2 of methanol molecule (total $-249.36 \mathrm{meV}=-243.15 \mathrm{meV}$ L-J $-6.17 \mathrm{meV}$ induction $-0.032 \mathrm{meV}$ electrostatic) and (c) orientation 3 of ethanol (total $-356.43 \mathrm{meV}=-348.78 \mathrm{meV} \mathrm{L}-$ $\mathrm{J}-7.09 \mathrm{meV}$ induction $-0.557 \mathrm{meV}$ electrostatic). On the other hand, the electrostatic potential is very small as compared to L-J potential and provides attractive or repulsive interactions depending on molecule orientations. In addition, the induction effects are relatively significant between polar and graphite because of the strong polarisability of polar molecule.

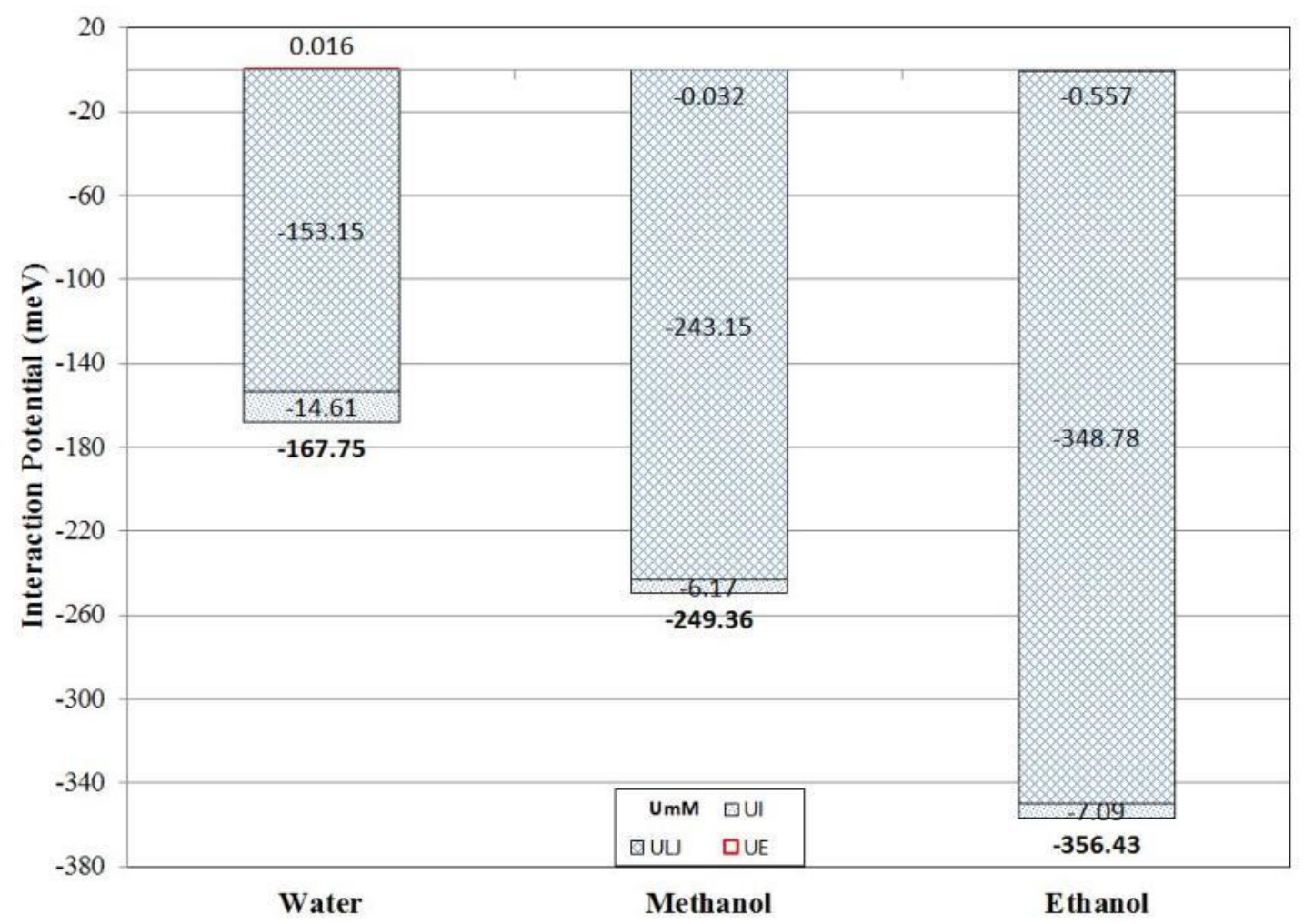

Figure 4.7 A detailed analysis on interaction potentials $v\left(z_{0}\right)$ for four polar molecules, where the orientations of $\mathrm{H}_{2} \mathrm{O}, \mathrm{CH}_{3} \mathrm{OH}$ and $\mathrm{CH}_{3} \mathrm{CH}_{2} \mathrm{OH}$ are chosen at their maximum $q_{s t}^{o}$ and $\mathrm{z}_{\mathrm{o}}$. 


\subsubsection{Interaction Potential of Silica with Water Molecule}

In the study of silica and water system, five orientations of water molecule above the amorphous $\mathrm{SiO}_{2}$ simulation box are shown in Figure 4.8.

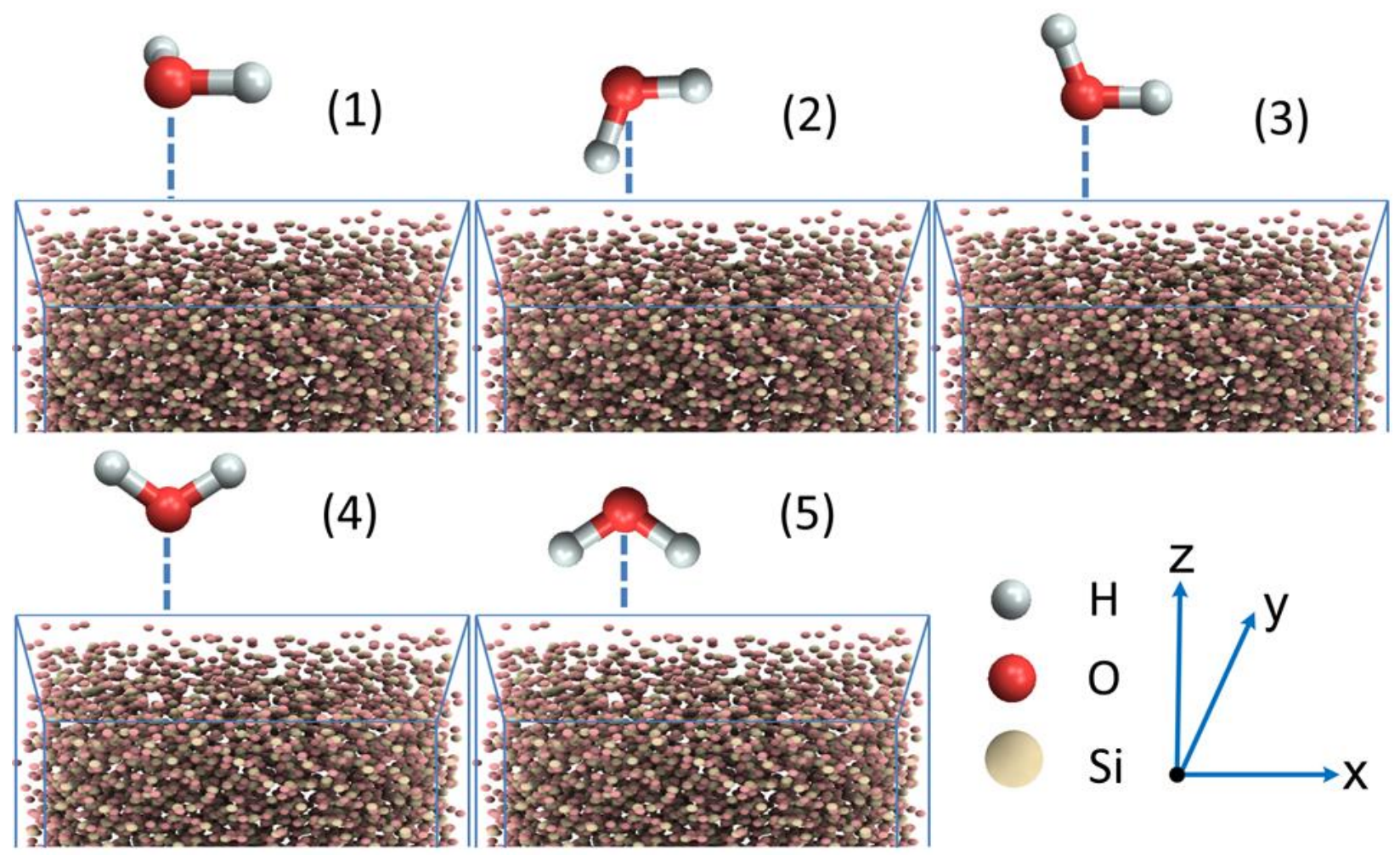

Figure 4.8 Five orientations of water molecule above top surface of amorphous silica

It should be noted here that each orientation and position of water molecule on the surface of silica pore contributes equally to the calculation of isosteric heat of adsorption. However, the average $q_{s t}{ }^{o}$ value can only be obtained by conducting an integration over an infinite set of all possible orientations and positions. Some orientations and positions are chosen to study their effects on both interaction potential and $q_{s t}{ }^{o}$ for identifying some favourable adsorptive sites and orientations of water molecule. For example, various orientations of water molecule are studied to predict the interaction potential between water molecule and graphite[135]. For orientations 1 to 5 , the rotation angles $\alpha, \beta$ and $\gamma$ of water molecule are varied up to $\left(0^{\circ}, 0^{\circ}, 0^{\circ}\right),\left(-90^{\circ}, 0^{\circ}, 0^{\circ}\right)$, $\left(90^{\circ}, 0^{\circ}, 0^{\circ}\right),\left(-90^{\circ}, 37.74^{\circ}, 0^{\circ}\right)$ and $\left(-90^{\circ}, 37.74^{\circ}+180^{\circ}, 0^{\circ}\right)$ while the distance between the 
centre of water molecule to the surface of silica is ranged from $2 \AA$ to $20 \AA$. Specifically, the H$\mathrm{O}-\mathrm{H}$ plane of water molecule is in $\mathrm{XY}$ plane for orientation 1 and the water molecule are rotated around $\mathrm{X}$ axis by $-90^{\circ}$ and $90^{\circ}$ for orientation 2 and 3. For orientation 4 , the water molecule is rotated around $X$ axis by $-90^{\circ}$ and then around $Y$ axis by $\frac{180^{\circ}-104.52^{\circ}}{2}=37.74^{\circ}$ For orientation 5 , the water molecule is rotated around $\mathrm{X}$ axis by $-90^{\circ}$ and then around $\mathrm{Y}$ axis by $180^{\circ}+37.4^{\circ}$, which yields the symmetry of orientation 4 and 5 about XY plane.

\section{Effect of orientation on potential with fixed position: variable $\alpha, \beta, \gamma$ and $z$}

In order to investigate the effect of various orientations on the interaction potential between water molecule and silica surface, the interaction potentials of water molecule with five orientations are calculated at the same fixed position where $\mathrm{x}=0 \AA$ and $\mathrm{y}=0 \AA$ while $\mathrm{z}$ can vary from around $1.5 \AA$ to $20 \AA$. The interaction potentials as a function of $\mathrm{z}$ for various orientations are shown in Figure 4.9 Two completely different trends of interaction potential are observed. Firstly, the interaction potential of all five orientations increase exponentially when the distance $\mathrm{z}$ decrease below maximum potential points and converge zero when $\mathrm{z}$ increases. Secondly, regardless of the positive region, there exists a maximum total interaction potential of $-0.258 \mathrm{eV}$, $-1.340 \mathrm{eV}$ and $-1.342 \mathrm{eV}$, where the attractive and repulsive force are equal, at $2.419 \AA, 2.073 \AA$ and $2.035 \AA$ for orientation 1,2 and 4 correspondingly. Thirdly, the interaction potential is positive and a monotonic decreasing trend for orientations 3 and 5 is observed, which indicates that the repulsive force is stronger than attractive force. Lastly, the comparison of potential component at $\mathrm{z}=4.0 \AA$ quantitatively characterizes the attraction and repulsion effect. Since the Lennard-Jones interaction of (Hydrogen $\cdots$ Silica) is usually ignored, the total Lennard-Jones potential remains unchanged here as the (Oxygen $\cdots$ Silica) distance for five orientations are the same when there is only rotation and no translation for the water molecule. 


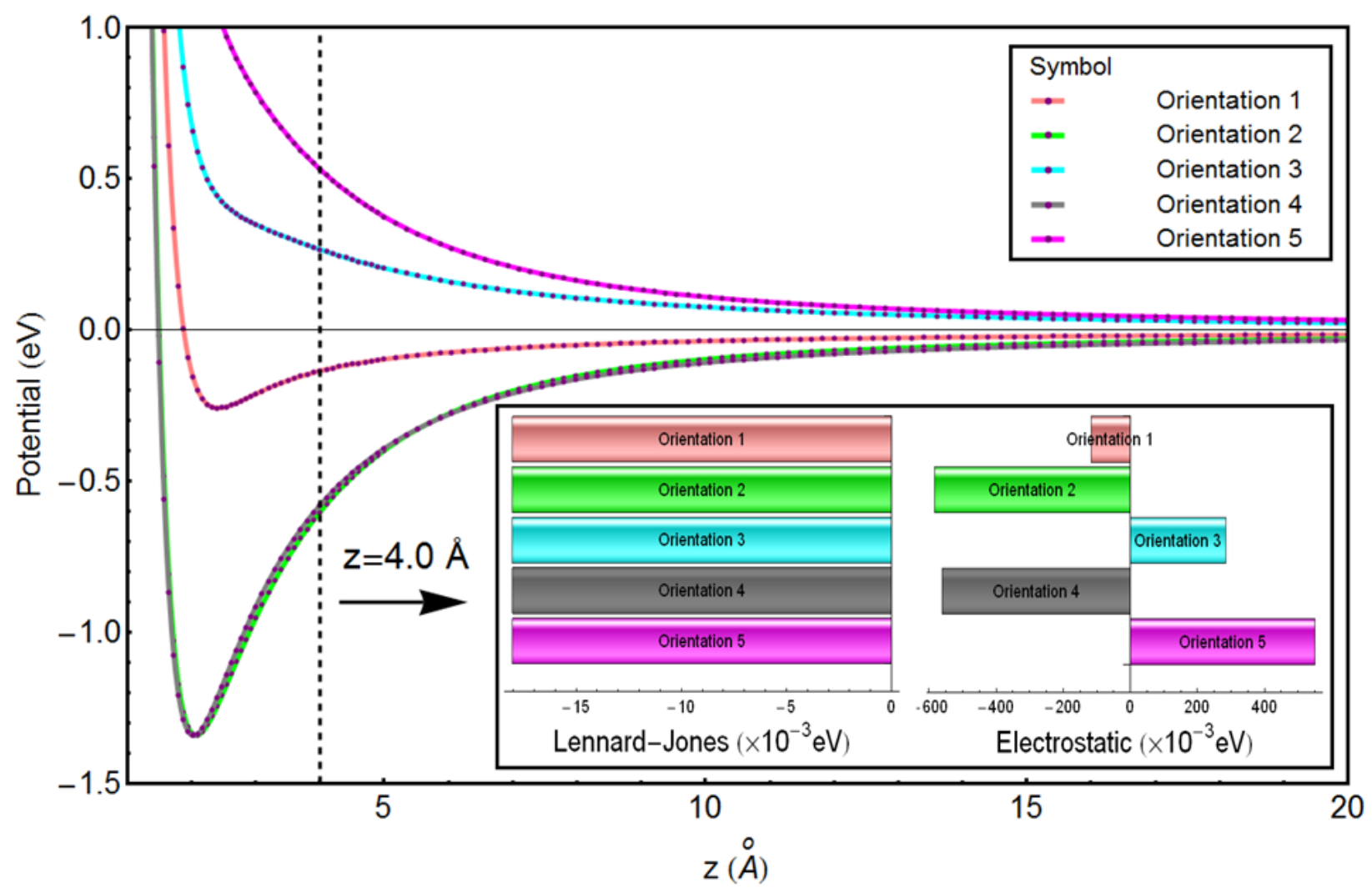

Figure 4.9 Interaction potential of water molecule of five orientations with the amorphous silica structure, where electrostatic potential is found significantly very high compared with LJ potential.

As the total interaction potential is significantly affected by the orientation, it is reasonable to conclude that the orientation will be changed to certain favourable orientations in the process of a water molecule being adsorbed on silica pore. These favorable orientations are determined by local geometrical characteristics of silica structure. Therefore, it is also necessary to identify these favourable adsorptive sites on silica surface.

\section{Effect of position on potential with fixed orientation: variable $\mathrm{x}$ and $\mathrm{z}$}

For the identification of favourable adsorptive sites with relatively stronger interaction, the contour plot of interaction potential for water molecule of orientation 1 on amorphous silica surface is shown in Figure 4.10 Here the $\mathrm{z}=2.65 \AA$, which is close to the maximum potential 
point. Regardless of the positive potential area, some favourable adsorptive sites with relatively larger interaction potential can be identified such as (i) $x=-11.5 \AA$ and $y=-11.5 \AA$, (ii) $x=-4 \AA$ and $\mathrm{y}=4 \AA$ and (iii) $\mathrm{x}=12.5 \AA$ and $\mathrm{y}=14.5 \AA$.

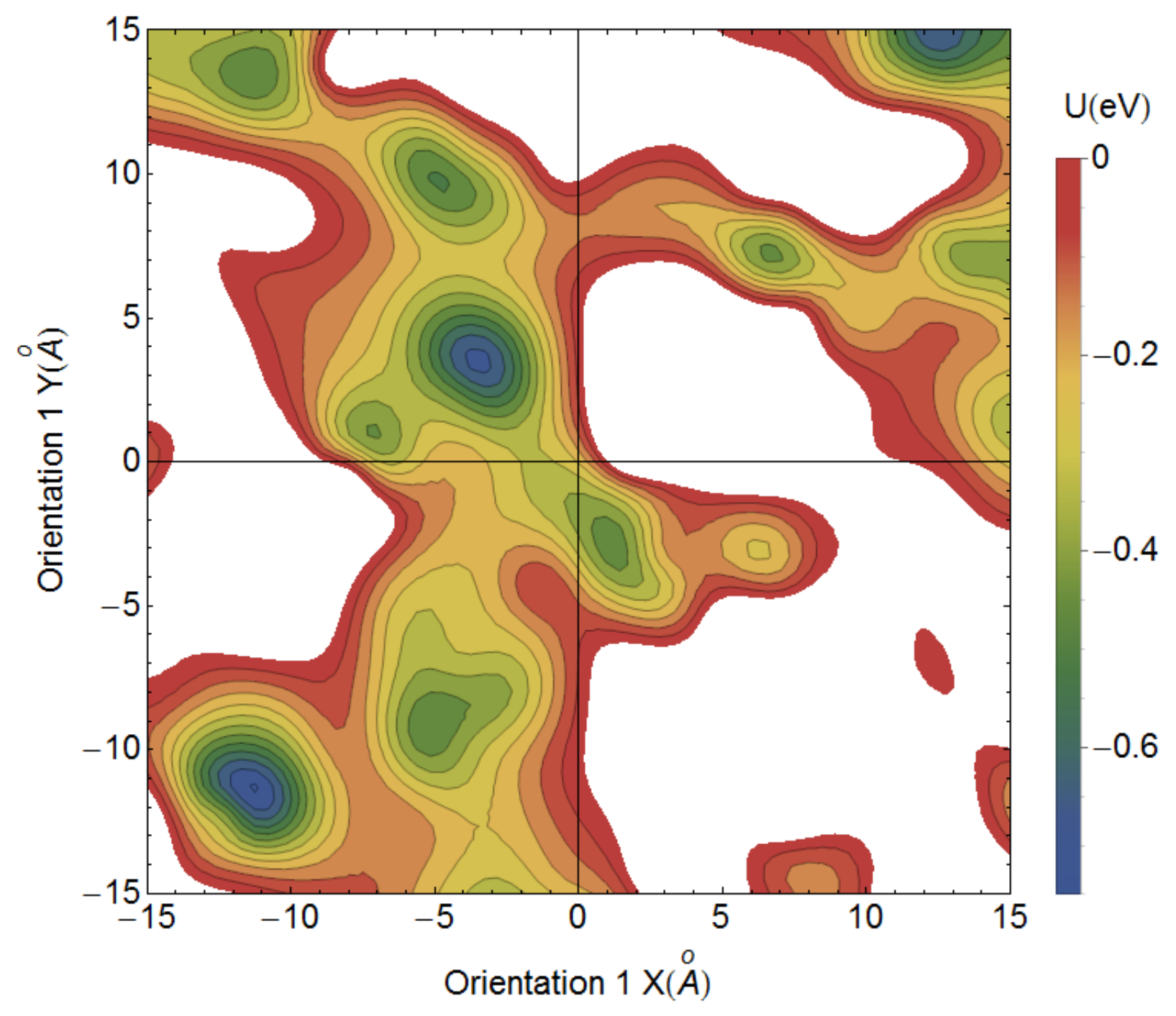

Figure 4.10 Interaction potential of water molecule in orientation 1 with the amorphous silica structure

In order to illustrate the change of interaction potential with water-silica distance $\mathrm{z}$, the water molecule of orientation 1 at five consecutive positions is further studied. Five positions are 
chosen in the simulation box due to the fact that the centre $(\mathrm{x}=-11.5 \AA$ and $\mathrm{y}=-11.5 \AA$ ) of Figure 4.10 is a favorable adsorptive site as the interaction potential can be found here.

Employing the concept of interaction potential, a plot of $\mathrm{XZ}$ plane $(\mathrm{y}=-11.5 \AA)$ is shown in Figure 4.11 It is found that the interaction potentials of five locations with respect to the $\mathrm{x}$ coordinate $(-11 \AA,-11.25 \AA,-11.5 \AA,-11.75 \AA$ and $-12 \AA)$, and the same $y=-11.5 \AA$ and $z=2.65 \AA$ vary from around $-0.7 \mathrm{eV}$ to $-0.75 \mathrm{eV}$.

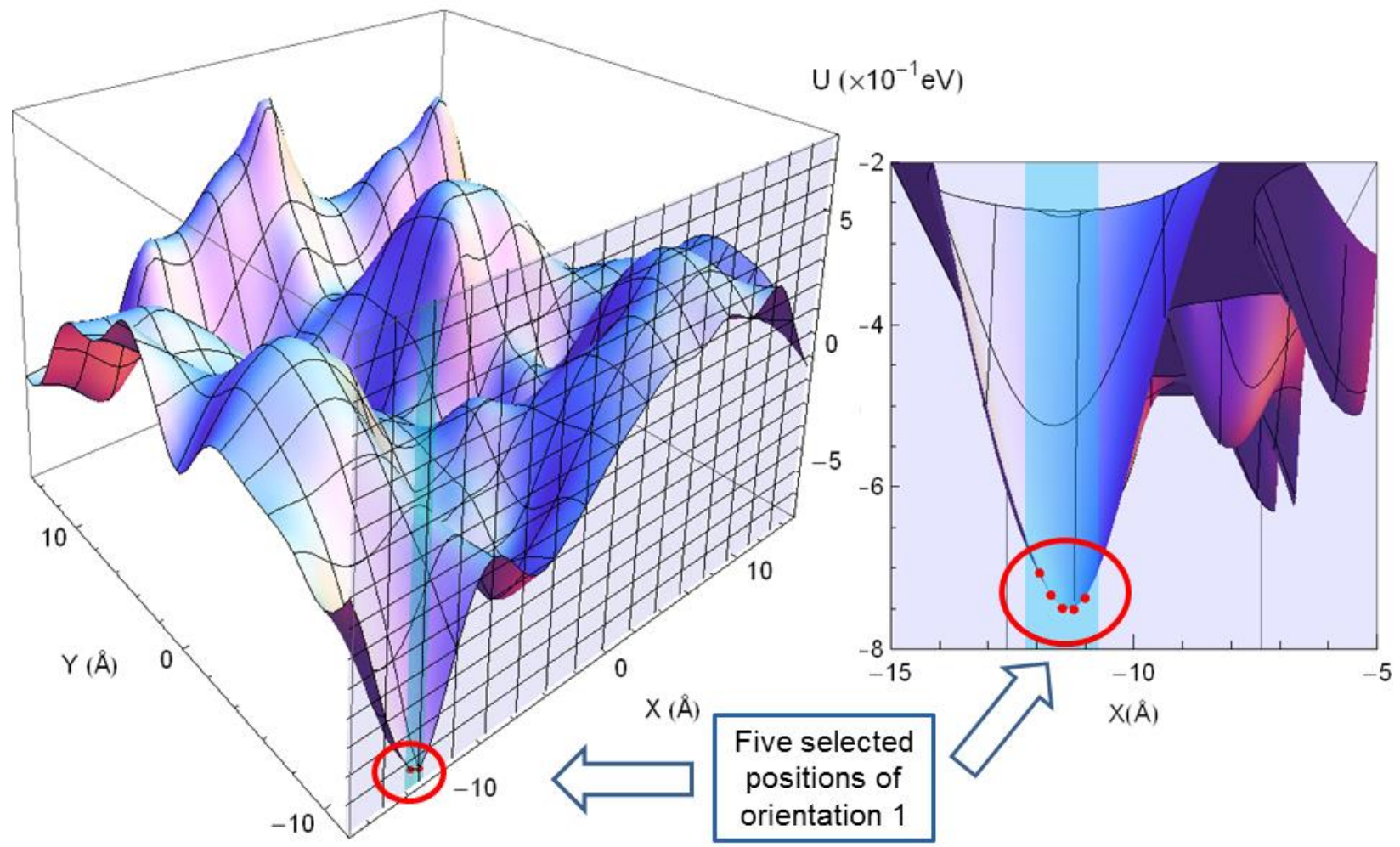

Figure 4.11 Illustration of choosing five positions of water in orientation 1 on the amorphous silica structure

The interaction potential of silica and water molecules at the five different positions of water molecules with respect to orientation 1 are shown in Figure 4.12. 


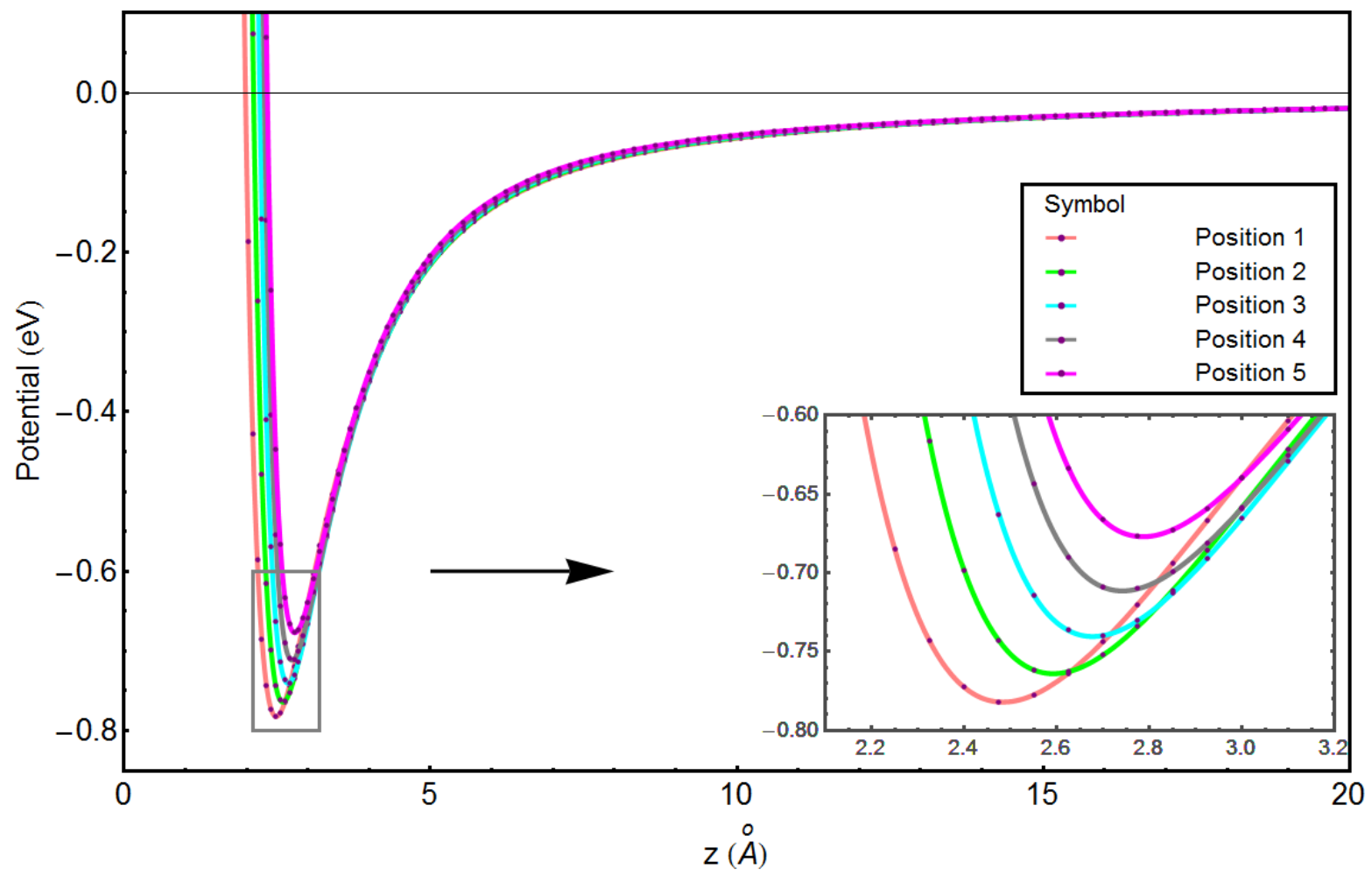

Figure 4.12 Interaction potential of water molecule of five positions in orientation 1 with the amorphous silica structure

Hence, the $\mathrm{x}$ coordinate varies from $-11 \AA,-11.25 \AA,-11.5 \AA,-11.75 \AA$ to $-12 \AA$ and $\mathrm{y}$ coordinate remains at $-11.5 \AA$, the maximum interaction potential for five different positions are found at $-0.782 \mathrm{eV},-0.764 \mathrm{eV},-0.741 \mathrm{eV},-0.712 \mathrm{eV}$ and $-0.677 \mathrm{eV}$ at $2.484 \AA$, $2.592 \AA, 2.677 \AA$,

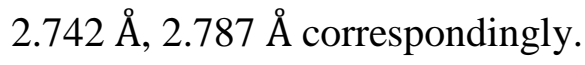

\section{Effect of position on potential with fixed orientation: variables $x, y$ and $z$}

Even though the sites with positive interaction potential are presented, some favorable adsorptive sites with relatively higher interaction potential are identified from the contour plots of negative interaction potential. These are shown in Figure 4.13. 

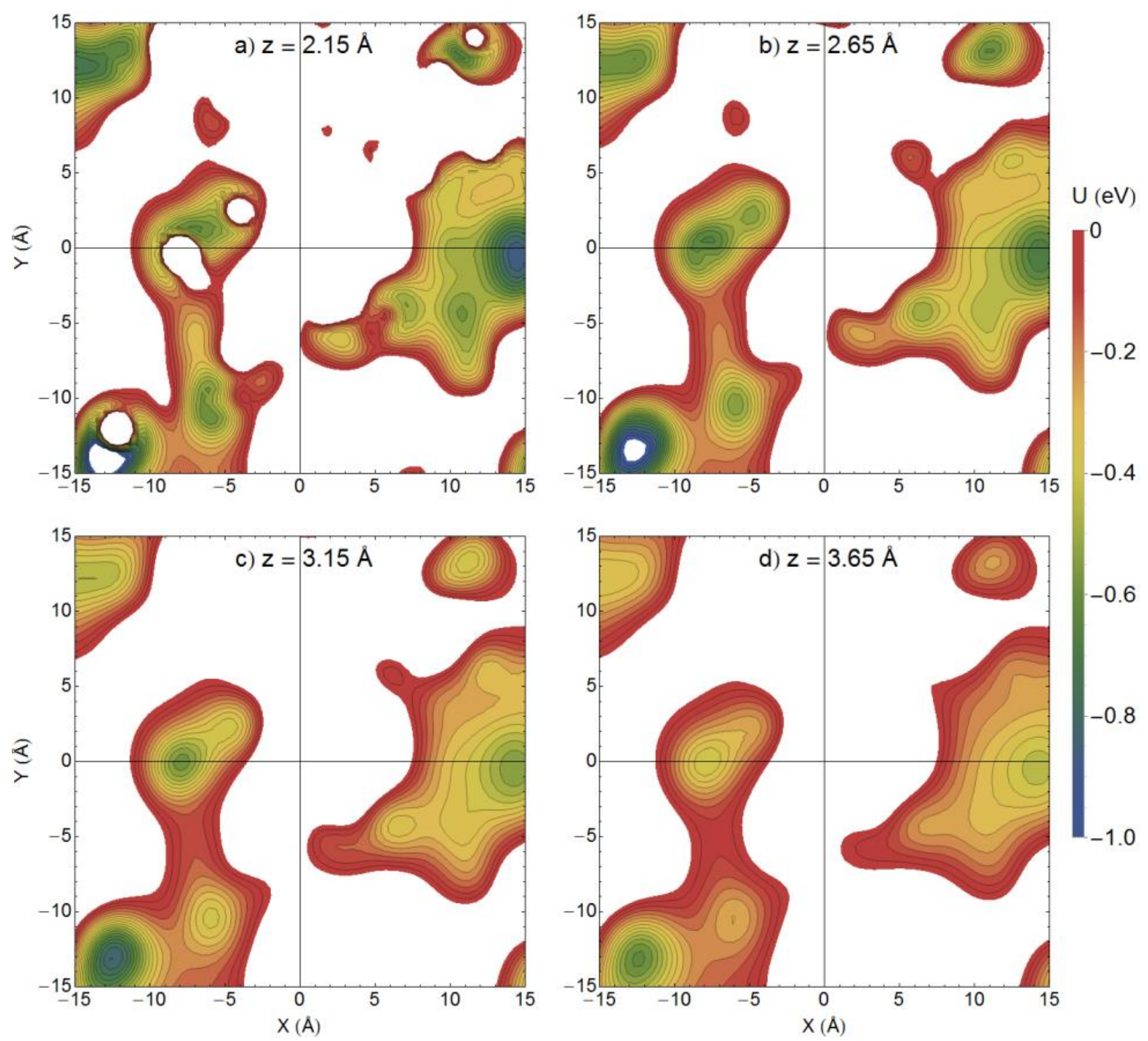

Figure 4.13 Interaction potential of water molecule at orientation 5 with distance of $2.15 \AA$, $2.65 \AA, 3.15 \AA$, and $3.65 \AA$ to the amorphous silica structure. The white portion indicates here the positive interaction potential.

The sites with negative interaction potential indicate that the attraction of silica surface to water molecule is stronger than repulsion. Employing the contour plot of interaction potential between water molecule and silica surface at different distances of $2.15 \AA$, $2.65 \AA, 3.15 \AA$ and $3.65 \AA$, it is found that the interaction potentials at certain favourable adsorptive sites change from negative 
to positive with the decrease of distance to silica surface. The interaction potentials at these sites are also decreased rather fast when the distance between water molecule and silica surface is increased. The maximum interaction potential at the most favorable adsorptive site decreases from $-1.244 \mathrm{eV},-1.036 \mathrm{eV},-0.855 \mathrm{eV}$ to $-0.700 \mathrm{eV}$ when the distance to silica surface increase from $2.15 \AA, 2.65 \AA, 3.15 \AA$, to $3.65 \AA$. This is caused by the exponentially increased LenardJones and electrostatic potential.

\subsubsection{Interaction Potential of Zeolites with Water Molecule}

The interaction potential of water molecules on CHA type zeolite is shown in Figure 4.14 for the distance of $2.8 \AA$ in $\mathrm{z}$ direction.
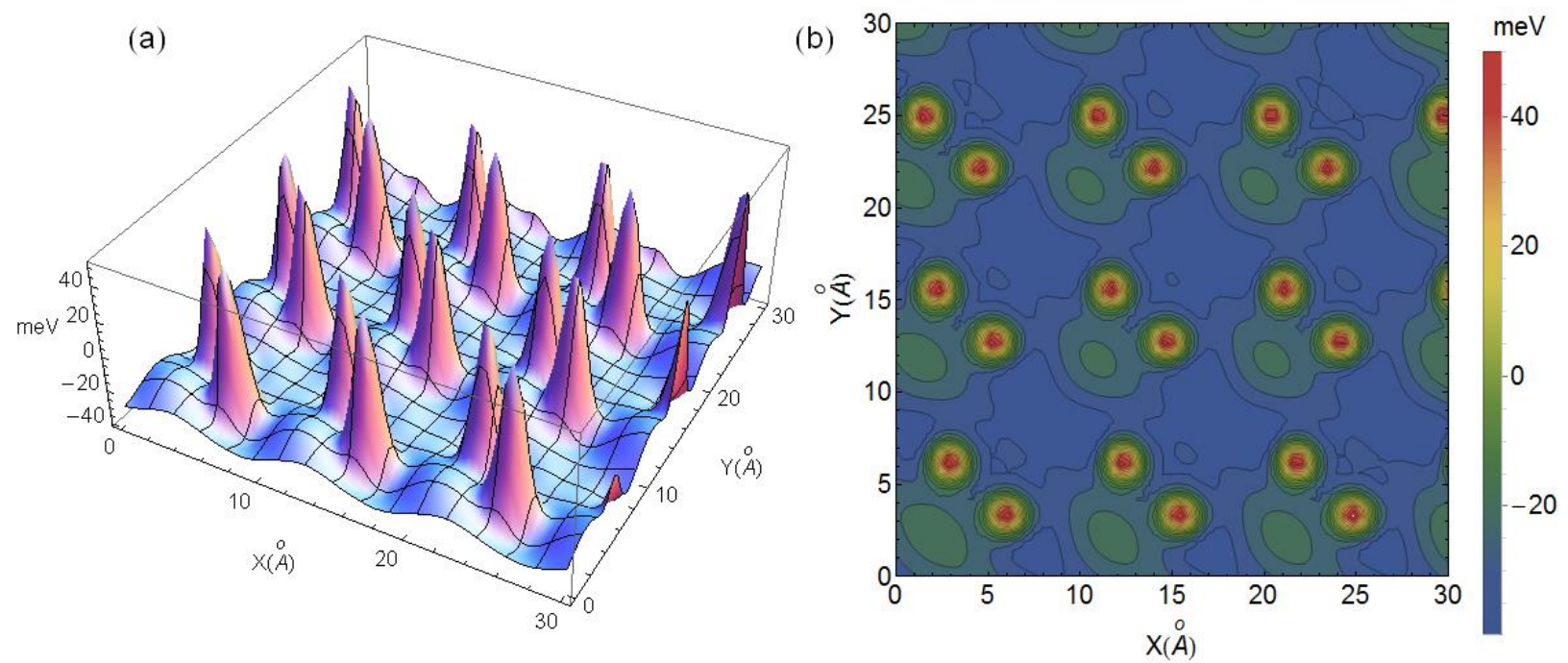

Figure 4.14 Interaction potential for the adsorption of water molecules on CHA zeolite employing (a) a 3 dimensional, and (b) contour plots.

From Figure 4.14, it is found that the interaction potential is highly negative sides, i.e., provides stronger attraction at certain periodic positions of zeolite framework, which indicates the positions of channels in the zeolite frameworks. Apparently, the water molecules are not adsorbed at those positions with higher interaction potential due to stronger repulsion between 
water molecules and zeolite surface. These most favourable adsorptive sites are the possible positions in which the water molecules are adsorbed.

The interaction potential of water molecule on the surface of AFI type zeolite is shown in Figure 4.15 for the distance of $1 \AA$ in the $\mathrm{z}$ direction.
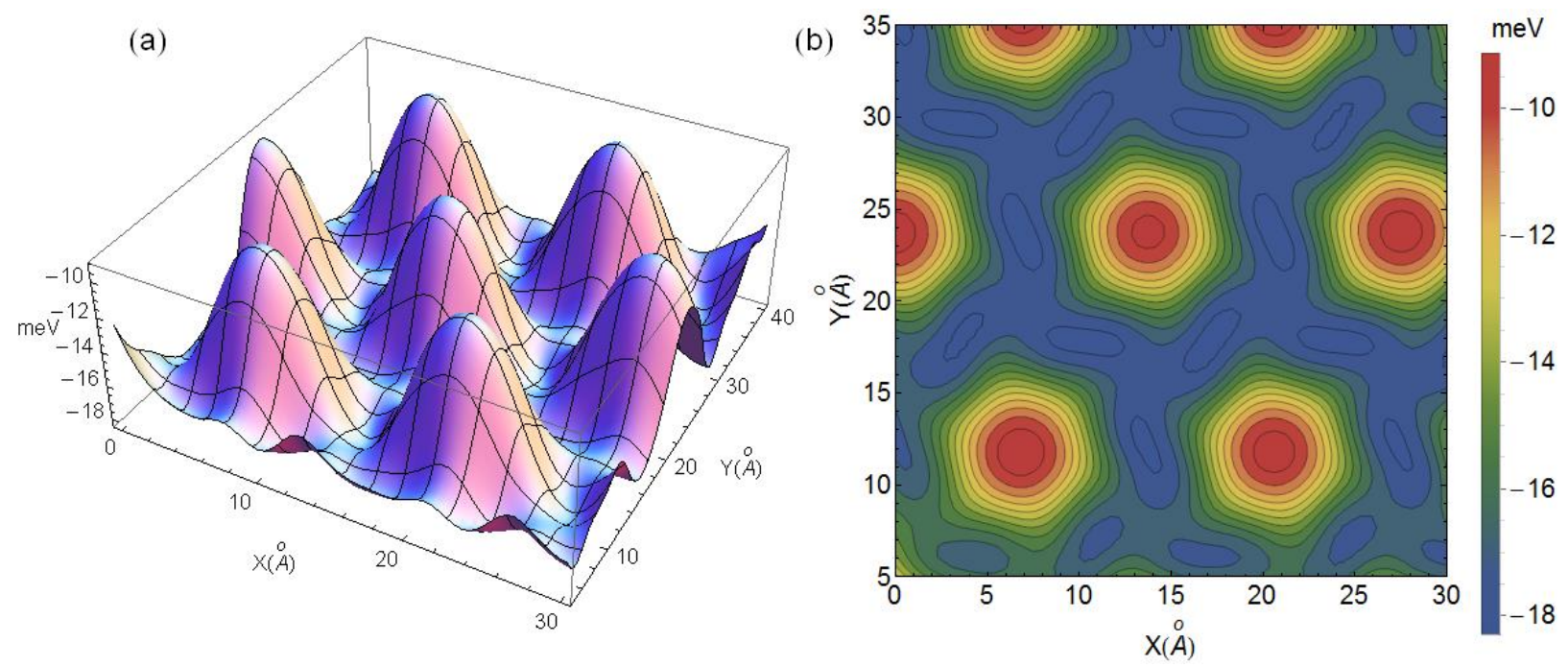

Figure 4.15 Interaction potentials of water molecule on AFI zeolite employing (a) a three

$$
\text { dimensional, and ( b) contour plots. }
$$

As compared with CHA type zeolite, the behaviours of periodic positions of interaction potentials as shown in Figure 4.15 are similar to the molecular structure of channels inside the zeolite frameworks, which also indicates that these channels possess the most favourable adsorptive sites for water molecule in the process of adsorption.

The interaction potential of water molecule and the cylindrical channel of CHA zeotype is shown in Figure 4.16. 


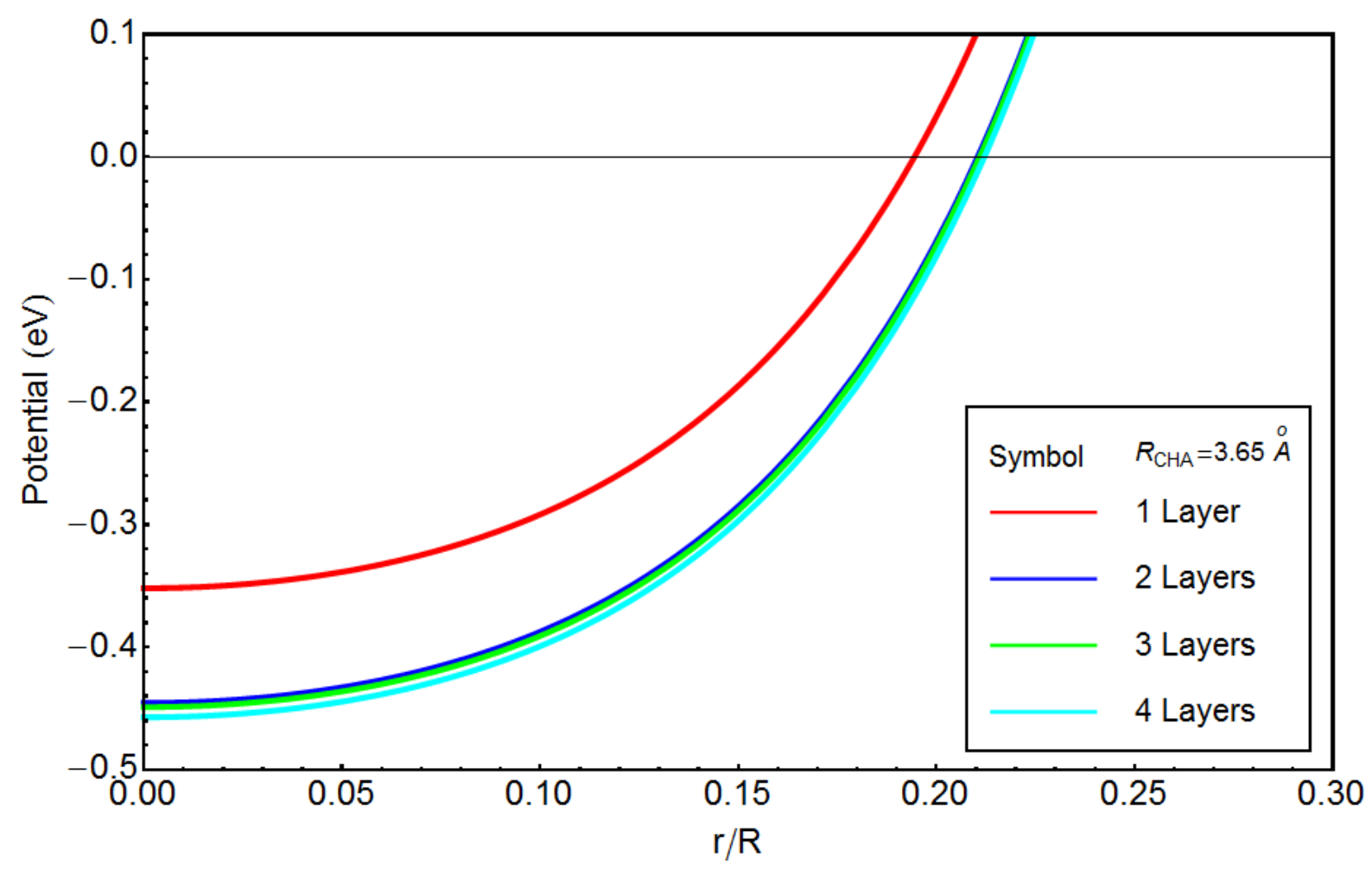

Figure 4.16 The interaction potential of a water molecule with the cylindrical channel inside CHA zeotype as a function of distance to the centre of the channel.

For CHA zeolite, it is found that the interaction potential is much negatively higher (stronger attraction) at the center of zeolite channel. Apparently, the water molecule will most probably not be adsorbed at those positions with positively higher infarction potential i.e. stronger repulsion to the zeolite surface of the channel. The most favorable adsorptive sites are found at the center of the channel. On the other hand, if multiple layers, other than only the first layer, are considered for interacting with one water molecule, the total interaction potential increases due to the additional interactions of $\mathrm{Si}$ and $\mathrm{O}$ atoms with water. The interaction potential increases strongly when the second layer is considered. The total potential is not significantly increased even though the effect of third layer is added in the modelling. This is due to the fact that in only $\mathrm{O}$ atoms are present in the third layer. The interactions between water molecule and the fourth 
layer of the cylindrical channel are very weak. Therefore, the increase in total potential (after addition of fourth layer's effects) is negligible.

The interaction potentials for the adsorption of water molecules on cylindrical channel inside AFI zeotype are shown in Figure 4.17

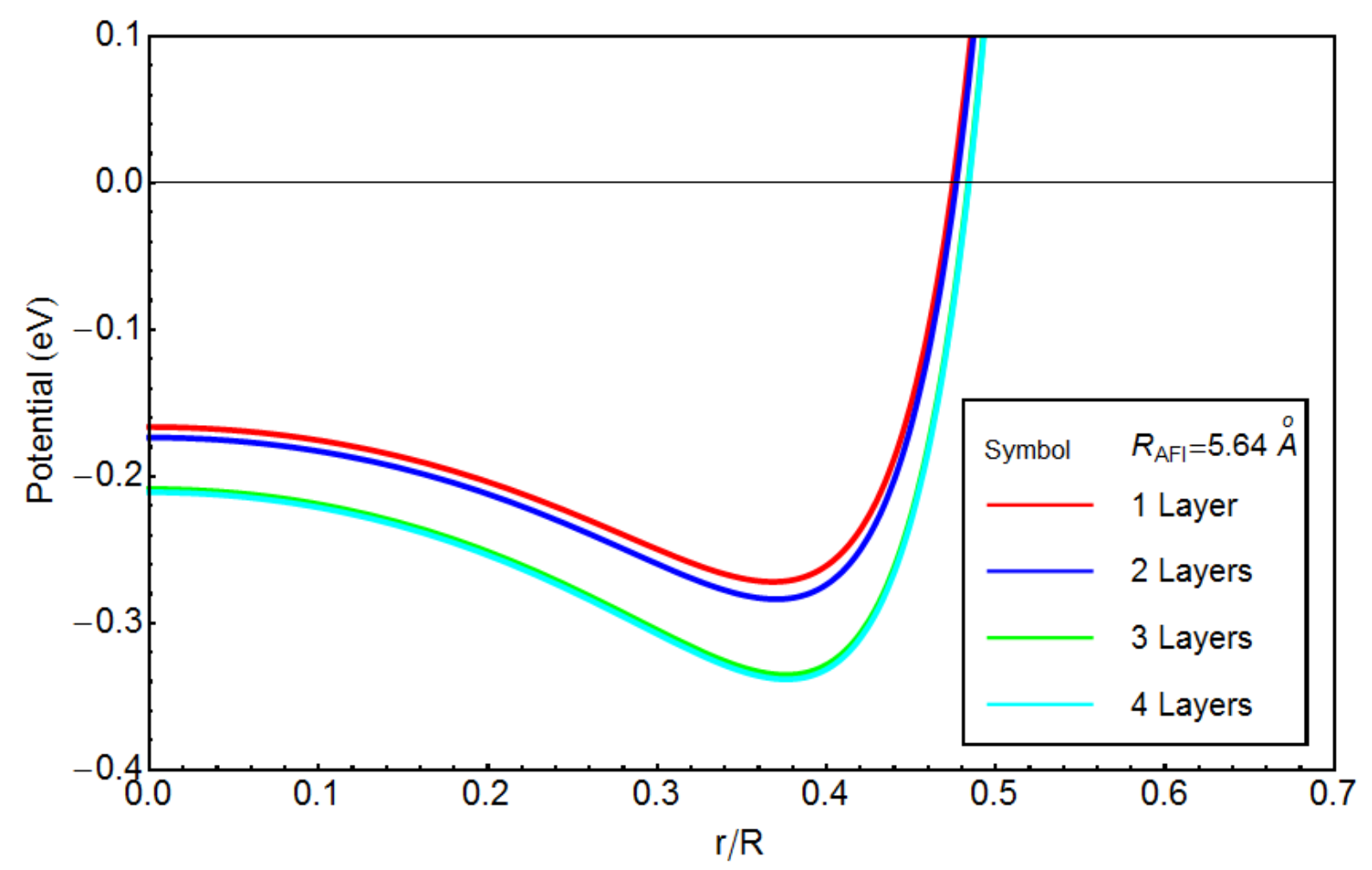

Figure 4.17 The interaction potential of a water molecule with the cylindrical channel inside AFI zeotype as a function of distance to the centre of the channel

For AFI zeolite, it is found that the maximum negative interaction potential positions are located not at the center of the channel but at $\mathrm{r} / \mathrm{R}$ of around 0.38 . Only $\mathrm{O}$ atoms are present in the second layer, and the increase in $w(r)$ is not very high. In case of the third layer, the $w(r)$ increases significantly due to the presence of $\mathrm{Al}, \mathrm{P}$ and $\mathrm{O}$ atoms. The distance from the center to the fourth layer is nearly $10 \AA$ and only oxygen atoms are present in the fourth layer. Therefore, the interactions between water molecule and the fourth layer of the cylindrical channel are very weak. 


\subsection{Results of Isosteric Heats of Adsorption}

\subsubsection{Isosteric Heats of Adsorption of Graphite with Water, Methanol and Ethanol}

\section{Molecues}

Employing the formulation of the previous chapter, plots of $q_{s t}^{o}$ for different water molecule orientations are shown in Figure 4.18.

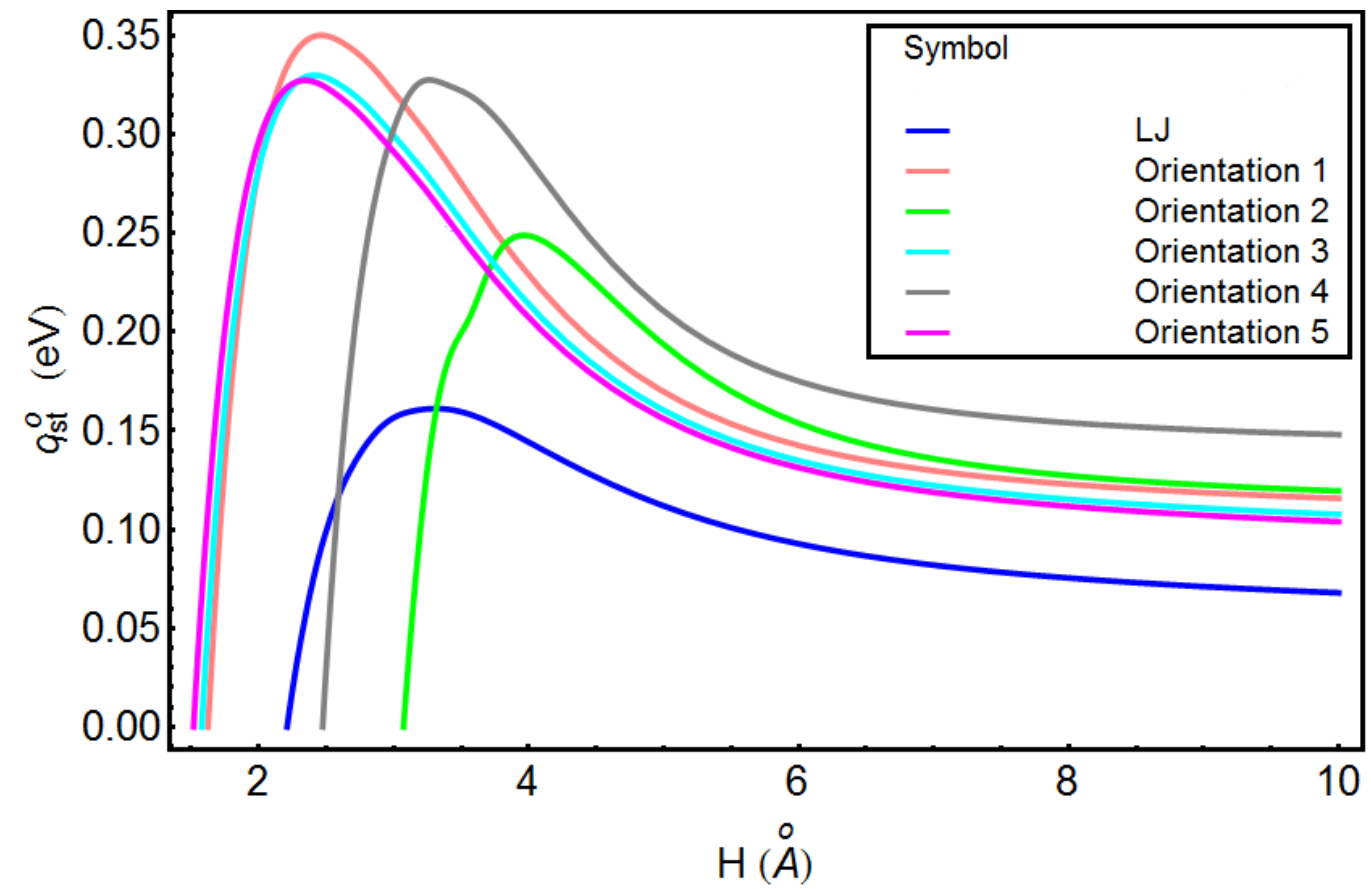

Figure 4.18 Isosteric heat of adsorption of single water molecule on graphite lattice at zero surface coverage as a function of $\mathrm{z}$ for the selected five $\mathrm{H}_{2} \mathrm{O}$ orientations

It is found that the maximum $q_{s t}^{o}$ of one molecule varies from $0.25 \mathrm{eV}$ to $0.35 \mathrm{eV}$. For maximum isosteric heat of adsorption, the pore width varies from $2 \AA$ to $4.5 \AA$ with respect to the water molecule orientations. The interaction energy between a water molecule and a single layer of graphite was estimated by Feller and Jordan [136] to be $5.8 \pm 0.4 \mathrm{kcal} / \mathrm{mol}(\approx 0.25 \mathrm{eV})$. In other reports $[137,138]$, the $q_{s t}^{o}$ values at zero surface coverage vary from $1.65 \mathrm{kcal} / \mathrm{mol}$ to 4.3 $\mathrm{kcal} / \mathrm{mol}(0.071 \mathrm{eV}$ to $0.185 \mathrm{eV})$. Employing polarisable potential model, Karapetian and Jordan [139] predicted the heat of adsorption of water molecule and graphite to be $2.50 \mathrm{kcal} / \mathrm{mol}(\approx$ 
$0.107 \mathrm{eV})$. In another study [140], it was found that the water molecule was located at least $3.5 \AA$ above the graphite surface for the maximum $q_{s t}^{o}$. Not only this, the adsorption energies on graphite surface are varied from $2.90 \mathrm{kcal} / \mathrm{mol}$ to $13.72 \mathrm{kcal} / \mathrm{mol}(\approx 0.125 \mathrm{eV}$ to $0.59 \mathrm{eV})$ for the distance of oxygen - graphite surface ranging from $3.01 \AA$ to $3.043 \AA$ [134]. These data are also added in Figure $4.18 q_{s t}^{o}$ is found to be below its liquefaction $(0.42 \mathrm{eV})$ heat due to the hydrophobic behaviour of the graphite surface. It should be noted here that the maximum $q_{s t}^{o}$ is found for the pore width ranging from $2.5 \AA$ to $4 \AA$, which can be compared with the mean diameter of water molecule $(2.9 \AA)$ or the hydrogen bond length of $3 \AA$.

The plots of $q_{s t}^{o}$ for various orientations of $\mathrm{CH}_{3}-\mathrm{OH}$ molecule on graphite surface are shown in Figure 4.19.

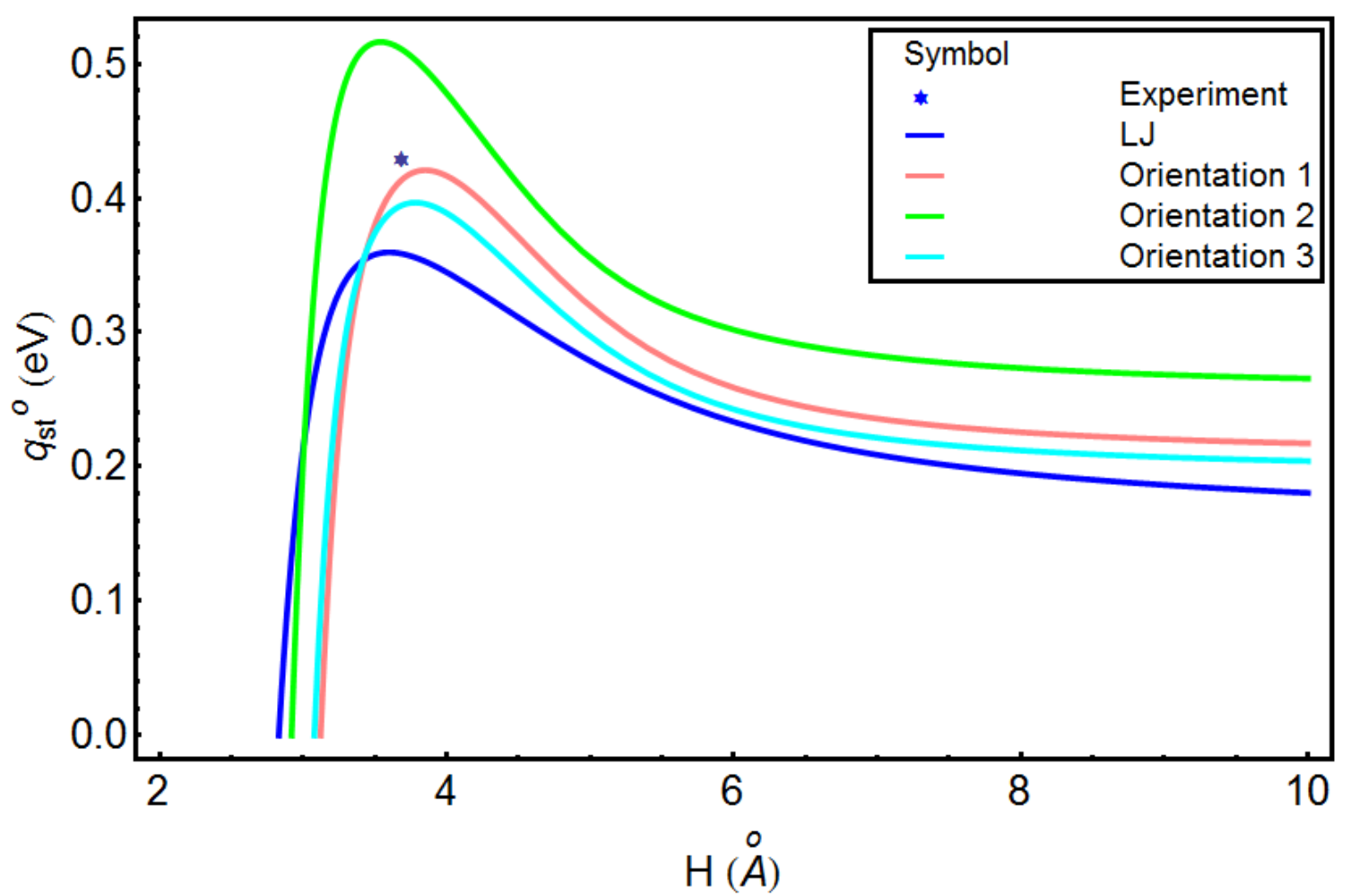

Figure 4.19 Isosteric heat of adsorption of single $\mathrm{CH}_{3}-\mathrm{OH}$ molecule on graphite lattice at zero surface coverage as a function of $\mathrm{z}$ for the selected three $\mathrm{CH}_{3}-\mathrm{OH}$ orientations 
The higher $q_{s t}^{o}$ at zero $\mathrm{CH}_{3}-\mathrm{OH}$ loading varies from $0.35 \mathrm{eV}$ to $0.44 \mathrm{eV}$ due to the combination of methanol-methanol and methanol - graphite interactions with the spill-over of methanol molecules onto the graphite surface. At the beginning of methanol adsorption on carbon pores, $q_{s t}^{o}$ is measured to be $58 \mathrm{~kJ} / \mathrm{mol}(\approx 0.58 \mathrm{eV})$ at $303 \mathrm{~K} \mathrm{[43].} \mathrm{For} \mathrm{higher} \mathrm{pore} \mathrm{width,} \mathrm{the} q_{s t}^{o}$ decreases due to weak interactions between $\mathrm{CH}_{3} \mathrm{OH}$ and graphite surface. As compared with the methanol molecule diameter of $4.2 \AA$, the maximum $q_{s t}^{o}$ is found for the values of $\mathrm{z}$ ranging from $3.4 \AA$ to $4 \AA$ with respect to various methanol molecule orientations. It is also found that at the distance of $3.7 \AA$, the attractive forces of the more distant groups of alcohol molecules are not compensated by the $\mathrm{r}$ epulsive forces and the $q_{s t}^{o}$ is measured by Avgul and Keslev [132] to be $10 \mathrm{kcal} / \mathrm{mol}(\approx 0.43 \mathrm{eV})$. The adsorption of methanol in graphite pores shows slightly different behaviour as compared to water due to stronger dispersion interactions of $\mathrm{CH}_{3}$ group with carbon surface. Horikawa et. al. [94] measured the $q_{s t}^{o}$ as $32 \mathrm{~kJ} / \mathrm{mol}(\approx 0.32 \mathrm{eV})$ for methanol adsorption on graphitised carbon block.

A plot of $q_{s t}^{o}$ for one $\mathrm{CH}_{3} \mathrm{CH}_{2} \mathrm{OH}$ molecule is found in Figure 4.20 


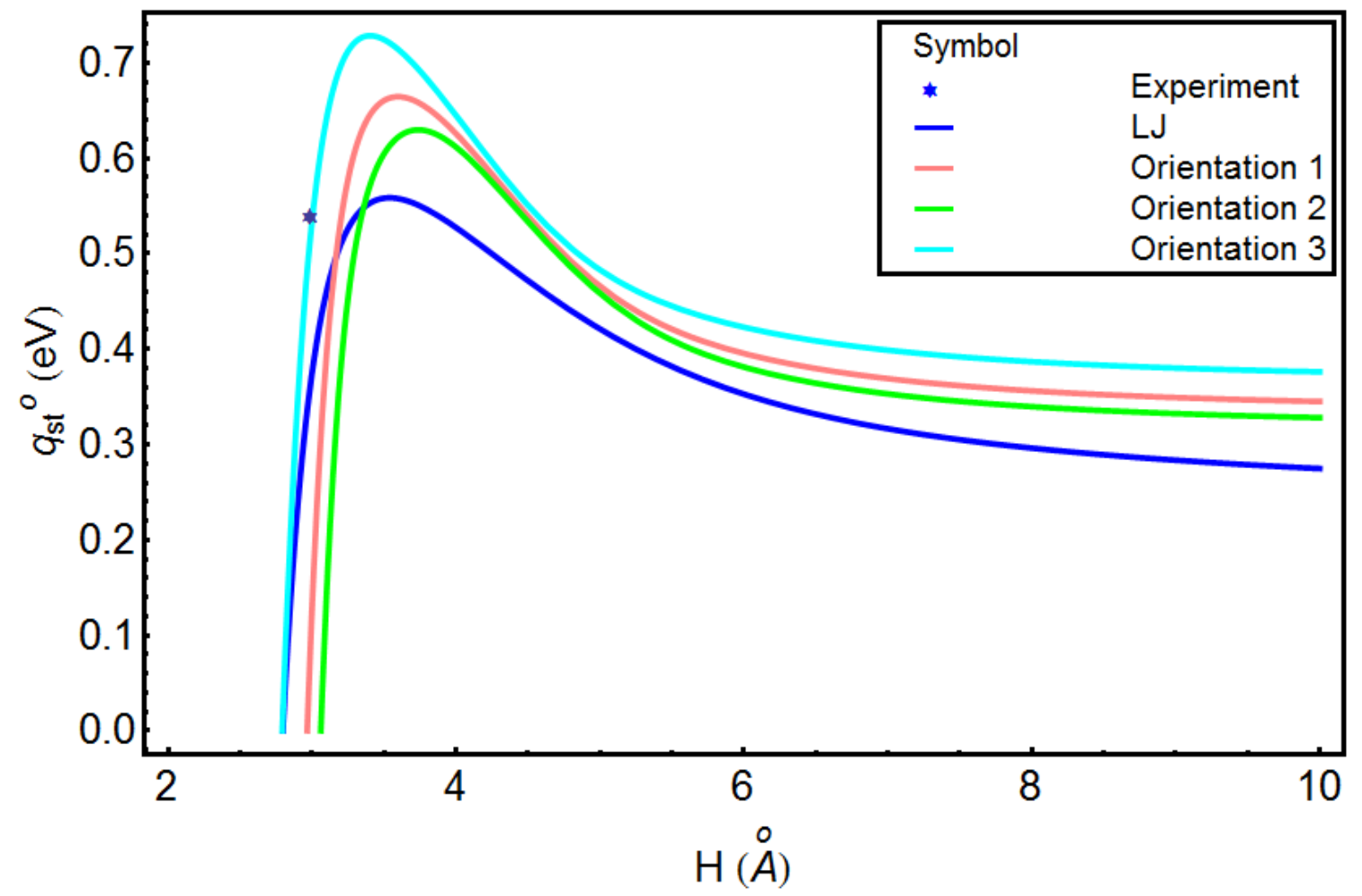

Figure 4.20 Isosteric heat of adsorption of single ethanol molecule on graphite lattice at zero surface coverage as a function of $\mathrm{z}$ for the selected three $\mathrm{CH}_{3} \mathrm{CH}_{2} \mathrm{OH}$ orientations

The $q_{s t}^{o}$ values at zero surface coverage varies from $0.56 \mathrm{eV}$ to $0.62 \mathrm{eV}$ for the orientations of ethanol molecule rotating from $90^{\circ}$ to $-90^{\circ}$. The maximum $q_{s t}^{o}$ is obtained between $3.5 \AA$ and 4 $\AA$ as similar to the ethanol molecule diameter of $4.4 \AA$. At the temperature of $300 \mathrm{~K}$, the simulation results of $q_{s t}^{o}$ at zero surface coverage are found in good agreement with the estimated experimental values that range from $13 \pm 0.4 \mathrm{kcal} / \mathrm{mol}(\approx 0.54 \mathrm{eV})$ to $16 \pm 0.4 \mathrm{kcal} / \mathrm{mol}(\approx 0.688$ eV) given by the reference[141]. From the simulation study, the optimum pore width is found to be $3.5 \AA$. This is due to the fact that at the distance of $3.5 \AA$, the attractive forces of isometric alcohol molecules are not compensated by the repulsive forces. 


\subsubsection{Isosteric Heats of Adsorption of Silica with Water Molecule}

The isosteric heat at zero coverage $\left(q_{s t}^{o}\right)$ for water adsorption on $\mathrm{SiO}_{2}$ is calculated as a function of pore width $(H)$. These results are presented in Figure 4.21, with respect to five different positions of water molecule on $\mathrm{SiO}_{2}$ surface.

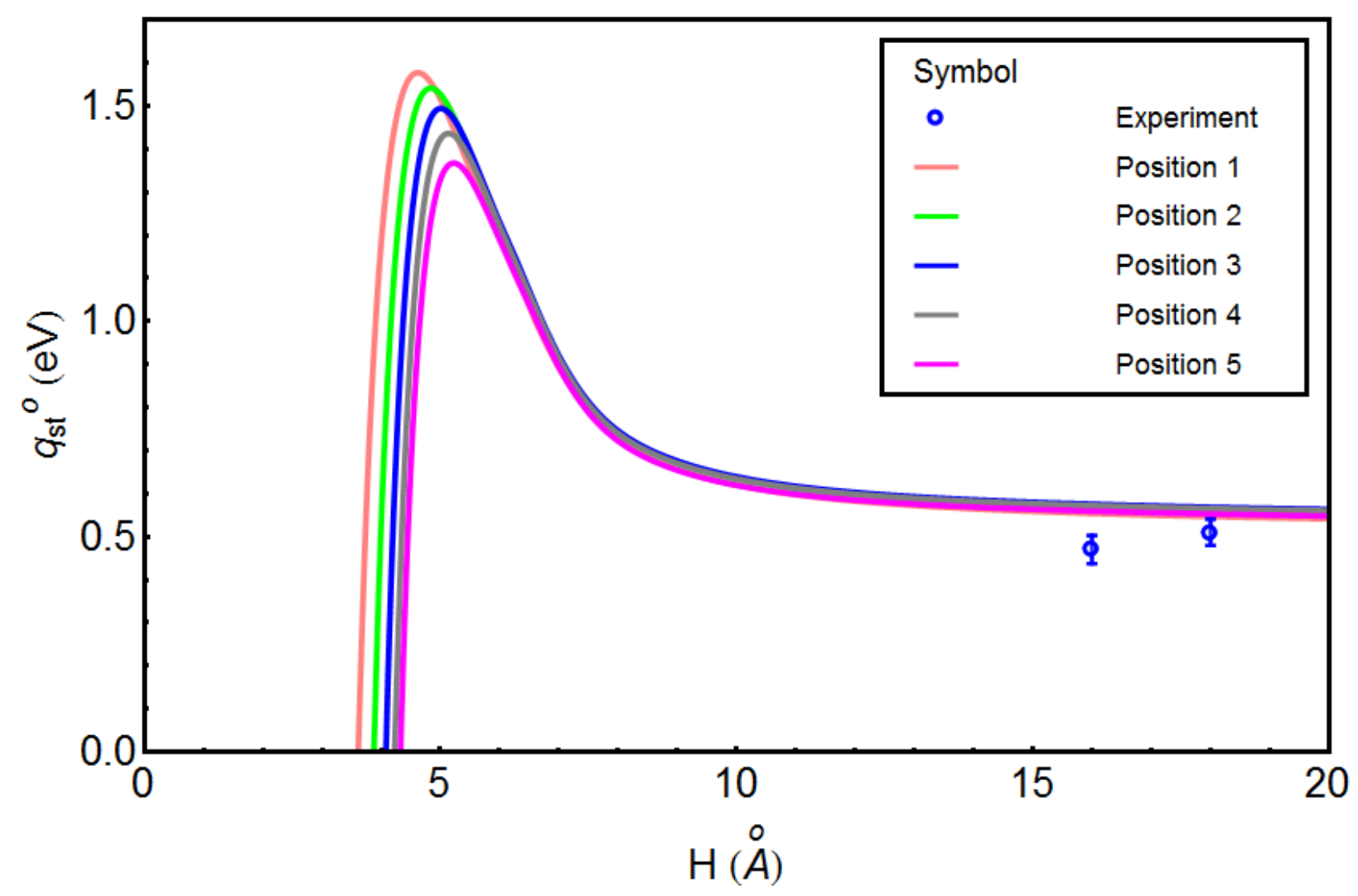

Figure 4.21 $q_{s t}^{o}$ of water of position 1 5 of orientation 1 on amorphous silica

The $q_{s t}^{o}$ values are increased from $4 \AA$ and the maximum values are found $1.578 \mathrm{eV}, 1.542 \mathrm{eV}$, $1.494 \mathrm{eV}, 1.436 \mathrm{eV}$ and $1.368 \mathrm{eV}$ for pore widths of $4.618 \AA$, $4.836 \AA$, $5.007 \AA$, $5.358 \AA$ and $5.223 \AA$ A depending on five positions of water molecule. Later, the $q_{s t}^{o}$ drops rapidly down to the transient region of pore width varying from $8 \AA$ and $13 \AA$. Between $13 \AA$ and $20 \AA$, all of the five $q_{s t}^{o}$ values converge around $50 \mathrm{~kJ} / \mathrm{mol}(\approx 0.52 \mathrm{eV})$ synchronously. The experimentally measured $q_{s t}$ data by Chua et al. are added in Figure 4.21 for comparison purposes. The $q_{s t}$ of types RD 
and A silica gel are calculated as $2510 \mathrm{~kJ} / \mathrm{kg}(\approx 0.47 \mathrm{eV})$ and $2710 \mathrm{~kJ} / \mathrm{kg}(\approx 0.51 \mathrm{eV})$ respectively [142].

The Grand Canonical Monte Carlo (GCMC) simulation is carried out to predict the $q_{s t}^{o}$ values of amorphous silica structure and water system. The $q_{s t}^{o}$ for the pore widths of $5 \AA$ and $10 \AA$ at the relative pressure of 0.05 is found to be $1.636 \mathrm{eV}$ and $1.942 \mathrm{eV}$. It can be found that GCMC simulation result is close to the maximum $q_{s t}{ }^{0}$ values at pore width of $5 \AA$ but the deviation increases for larger pore width. The isosteric heat of $\mathrm{CaCl}_{2}$-in-silica gel+ water system at low uptake of $0.05 \mathrm{~kg} / \mathrm{kg}$ is found to be around $3000 \mathrm{~kJ} / \mathrm{kg}(\approx 0.56 \mathrm{eV})[143]$

\subsubsection{Isosteric Heats of Adsorption at Zero Coverage for Zeolites with Water Molecule}

The simulation results of the isosteric heat at zero coverage employing two types of interaction potential equations are presented here.

With a variable pore size assumption, the simulation results of the isosteric heat at zero coverage for water adsorption on CHA and AFI types zeolite frameworks are employing cylindrical interaction potential equation is shown in Figure 4.22. For simplicity, both of the zeolite frameworks of two zeotypes are considered as layers of $\mathrm{T}$ and $\mathrm{O}$ atoms $(\mathrm{T}=\mathrm{Al}, \mathrm{P}$ for $\mathrm{AFI}$ structure or $\mathrm{T}=\mathrm{Si}$ for $\mathrm{CHA}$ structure) and the total interaction potential is obtained as introduced in Chapter 3. 


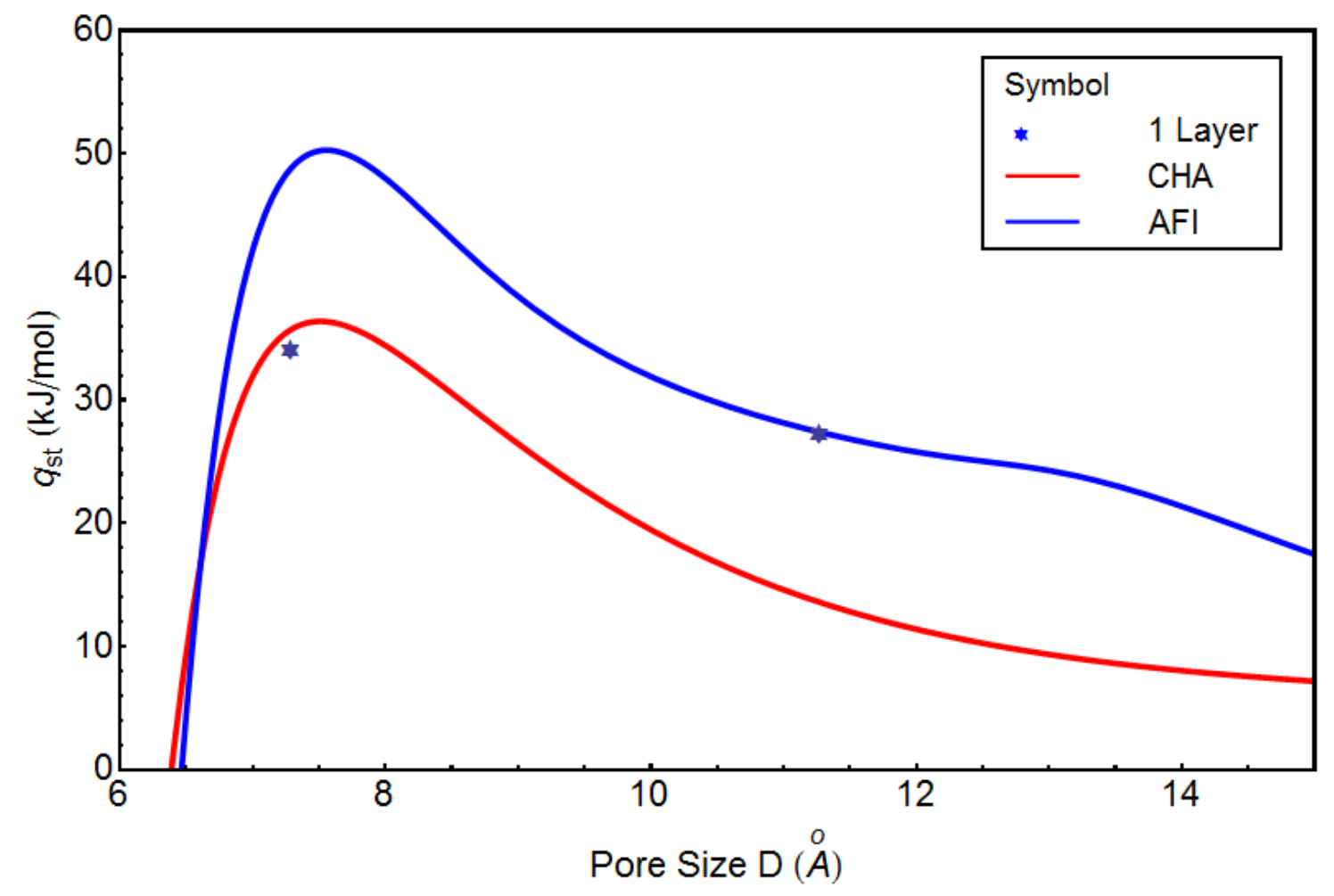

Figure 4.22 Isosteric heat of adsorption of water on zeolite of CHA and AFI framework type Employing equations in Chapter 3, the values of $q_{s t}^{o}$ as a function of pore width are calculated, and these results are shown in Figure 4.22, which considers only the micro-pore ranges ( $<20 \AA)$ and the first layer of CHA and AFI zeolites. The isosteric heats for first layers are found to be (i) $34.22 \mathrm{~kJ} / \mathrm{mol}$ for CHA (AQSOA-Z02) type zeolite at the pore diameter of $7.3 \AA$ and (ii) 27.37 $\mathrm{kJ} / \mathrm{mol}$ for AFI (AQSOA-Z01 and Z05) type zeolite at the pore diameter of $11.28 \AA$. These two values are also shown in Figure 4.22. At multiple layers, the $q_{s t}^{o}$ values are found higher due to additional interaction potentials of other layers comprising $\mathrm{Si}$ and $\mathrm{O}$ atoms for CHA type and $\mathrm{Al}$, $\mathrm{P}$ and $\mathrm{O}$ atoms for AFI type zeolites. For example, up to 4 layers, the isosteric heats for CHA and AFI zeolites are found to be $44.39 \mathrm{~kJ} / \mathrm{mol}$ and $33.77 \mathrm{~kJ} / \mathrm{mol}$, respectively. 


\subsection{Summary of Chapter 4}

\subsubsection{Graphite and Water, Methanol and Ethanol Molecules}

The interaction potential curves as a function of adsorbate - adsorbent separation distance $\mathrm{z}$ and $q_{s t}^{o}$ curves as a function of pore width $\mathrm{H}$ illustrate how these molecules interact with a graphite surface. Some important features are as follows:

(i) The maximum potential well for graphite - water system constitutes $91.28 \%$ Lennard Jones potential $\operatorname{vLJ}\left(\mathrm{C} \cdots \cdot \mathrm{H}_{2} \mathrm{O}\right), \quad 8.7 \%$ induction potential $\operatorname{vI}\left(\mathrm{C} \cdots \cdot \mathrm{H}_{2} \mathrm{O}\right)$ and $0.012 \%$ electrostatic $\mathrm{vE}\left(\mathrm{C} \cdots \mathrm{H}_{2} \mathrm{O}\right)$. The maximum potential well depth depends on various orientations of $\mathrm{H}_{2} \mathrm{O}$ molecule on graphite structure. The maximum $q_{s t}^{o}$ at zero surface coverage is found to be 0.35 $\mathrm{eV}$ at orientation 1 , where the oxygen atom is at the origin, one $\mathrm{H}$ atom is at the $\mathrm{X}$-axis and $\mathrm{H}-$ $\mathrm{O}-\mathrm{H}$ is obtained in the $\mathrm{X}-\mathrm{Y}$ plane, and the simulation result is very close to the experimental data of $0.32 \mathrm{eV}$. For water molecules, the electrostatic contribution for $\mathrm{v}(\mathrm{z})$ is very close to zero and provides attractive or repulsive interactions between adsorbate and graphite molecules.

(ii) For ethanol and methanol, the electrostatic contribution is close to zero and provides both attractive and repulsive interactions. The $\operatorname{vLJ}\left(\mathrm{C} \cdots \mathrm{CH}_{3}-\mathrm{OH}\right)$ and $\operatorname{vLJ}\left(\mathrm{C} \cdots \mathrm{CH}_{3}-\mathrm{CH}_{2}-\mathrm{OH}\right)$ contributions are more than $90 \%$. The induction interactions are less than $10 \%$. For both methanol and ethanol molecules, the induction potential increases exponentially for the separation distance decreasing from $3 \AA$ to 0 . However, the electrostatic potential remains constant and the value is very close to zero.

\subsubsection{Amorphous Silica and Water}

The structure of amorphous silica is constructed using the Reverse Monte Carlo (RMC) method. The radial and angle distribution functions together with the structure factor of the generated structure is compared with experimentally measured results to verify this molecular model. With 
the validated silica structure, the intermolecular potential between water and silica that integrating $\mathrm{LJ}$ and electrostatic potential are determined and applied in the calculation of isosteric heat of adsorption at zero surface coverage.

(i) The partial radial distribution and structure factor of the reference silica structure constructed by the Reverse Monte Carlo method are calculated to confirm the validity of $\mathrm{SiO}_{2}$ structure for $q_{s t}{ }^{o}$ calculation. Furthermore, the bond angle (O-Si-O and $\left.\mathrm{Si}-\mathrm{O}-\mathrm{Si}\right)$ distribution functions of the silica structure are calculated. The O-Si-O and Si-O-Si angles are mainly around $104^{\circ} \sim 112^{\circ}$ and $130^{\circ} \sim 145^{\circ}$ and are in good agreement with other simulation results while the most probable bond angle (MPBA) of Si-O-Si is found $146^{\circ}$ and that of $\mathrm{O}-\mathrm{Si}-\mathrm{O}$ is $109.4^{\circ}$.

(ii) The interaction potential is also determined by the water-silica distance $\mathrm{z}$ according to the interaction potential of five positions in orientation 1, which are chosen in the simulation box due to the fact that the centre $\mathrm{x}=-11.5 \AA$ and $\mathrm{y}=-11.5 \AA$ is a favourable adsorptive site due to the presence of the negative interaction potential. While the $\mathrm{x}$ coordinate is varied from $-11 \AA$ to -12 $\AA$ and y coordinate is remained fixed at $-11.5 \AA$, the maximum interaction potential ranges from $-0.782 \mathrm{eV}$ to $-0.677 \mathrm{eV}$.

\subsubsection{Zeolite and Water}

The isosteric heat of adsorption for water on three CHA and AFI types zeolites such as AQSOAZ01, AQSOA-Z02 and AQSOA-Z05 are studied. The following findings are obtained.

(i) The most favourable adsorptive sites with negatively larger potentials are identified at the positions of channels inside the zeolite framework.

(ii) The isosteric heat of adsorption of water adsorption on first layer of CHA and AFI types zeolites are predicted to be $34.22 \mathrm{~kJ} / \mathrm{mol}$ for CHA (AQSOA-Z02) type zeolite at the pore diameter of $7.3 \AA$ and $27.37 \mathrm{~kJ} / \mathrm{mol}$ for AFI (AQSOA-Z01 and Z05) type zeolite at the pore 
diameter of $11.28 \AA$. At multiple layers, the $q_{s t}^{o}$ values are found higher due to additional interaction potentials of other layers comprising $\mathrm{Si}$ and $\mathrm{O}$ atoms for CHA type and $\mathrm{Al}, \mathrm{P}$ and $\mathrm{O}$ atoms for AFI type zeolites. For example, up to 4 layers, the isosteric heats for CHA and AFI zeolites are found to be $44.39 \mathrm{~kJ} / \mathrm{mol}$ and $33.77 \mathrm{~kJ} / \mathrm{mol}$, respectively.

The isosteric heat at zero coverage is essential for the calculation of energetic performances of adsorption assisted cooling and desalination. In that case the isotherms data are needed for device simulation purposes. The next chapter will discuss about the experimental study of adsorption isotherms for silica gel + water, various zeolites + water and activated carbon $+\mathrm{CO}_{2}$, $\mathrm{CH}_{3}-\mathrm{OH}$ or $\mathrm{CH}_{3}-\mathrm{CH}_{2}-\mathrm{OH}$ systems. 


\section{Chapter 5 Measurement of Adsorption Uptakes under Static}

\section{Conditions}

Evaluation of the amount of water vapour on silica gel and AQSOA-type zeolites for various temperatures and pressures is needed to design an adsorption chiller for air conditioning and desalination applications. On the other hand, the amounts of $\mathrm{CO}_{2}$ uptakes on activated carbons have to be determined for the design of adsorption refrigeration system. In this chapter, an experimental study for calculating adsorbate uptakes is described.

\subsection{Experimental Investigation}

\subsubsection{Measurement of $\mathrm{CO}_{2}$ Uptakes on Activated Carbon}

The amount of $\mathrm{CO}_{2}$ uptake was determined by the Control Volume Variable Pressure (CVVP) method as shown schematically in Figure 5.1 The CVVP apparatus consists of three sections, $\mathrm{CO}_{2}$ storage cylinder, charging and adsorption chambers. The charging section provides a calibrated space for $\mathrm{CO}_{2}$ to charge into, where the initial mass of $\mathrm{CO}_{2}$ gas can be measured. The adsorption section offers an adsorption site for the adsorbent + adsorbate pair. The volume of both adsorption and charging sections are calibrated with water and are recorded as $V_{\text {rec }}$ and $V_{\text {char }}$, respectively. The charging and adsorption chambers are heated with a hot water bath to ensure the same constant temperature during the whole adsorption process. All the pipes are covered with heating wire and insulation to maintain a target temperature with an adjustable heater in the experiment. The adsorption cell had been designed with a high aspect ratio, so that the adsorbent could be spread on the large flat base. Pressure transducers (type Kyowa-PGS200KA, uncertainty of $0.2 \%$ full scale) are used to measure the pressures of charging and adsorption cells. The temperature of charging chamber was recorded by class-A Pt $100 \Omega$ RTDs 
with uncertainty of $\pm 0.15^{\circ} \mathrm{C}$. The temperature of adsorption chamber was controlled by the DT670B-CU-HT silicon diode thermometers with the uncertainty of $\pm 0.1^{\circ} \mathrm{C}$.

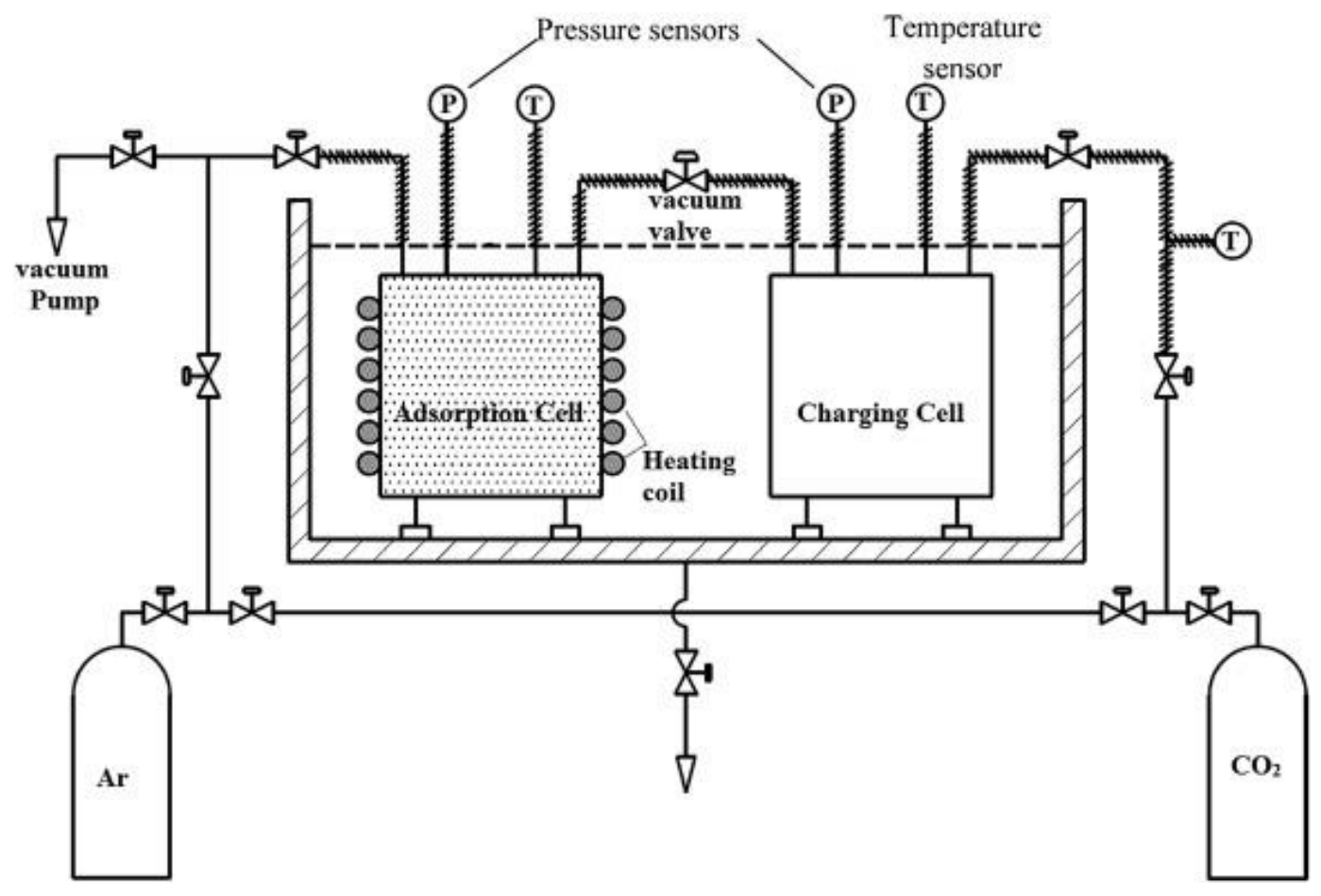

Figure 5.1 Schematic diagram of CVVP apparatus for measuring $\mathrm{CO}_{2}$ adsorption of activated carbons.

The pressure within the adsorption chamber was measured by GS4200-USB digital pressure transducer with an error of $\pm 0.1 \%$. Before the experiments, the adsorbent sample was dried in an oven at $420 \mathrm{~K}$ for $6 \mathrm{~h}$ and then placed in the adsorption chamber after calculating its dry mass. The volume occupied by carbon sample within adsorption chamber has been taken into account by helium/argon gas calibration. Both the charging and adsorption cells were evacuated using a rotary vane vacuum pump (Brand: VACUUBRAND, Model: RC 6 ) for $6 \mathrm{~h}$ and the temperature of adsorption cell was kept at $420 \mathrm{~K}$ for regeneration. Prior to the first charging of $\mathrm{CO}_{2}$, the temperature of the charging cell was kept at $298 \mathrm{~K}$, while the adsorption cell temperature was ranged from $298 \mathrm{~K}$ to $363 \mathrm{~K}$ depending on experimental conditions. A certain amount of $\mathrm{CO}_{2}$ 
was then dosed into the charging cell. At the first step of the experiment, there was residue gas inside the adsorption cell and the mass of residue gas was calculated by $m_{r e c}=V_{r e c} \rho_{r e c}$, where $V_{r e c}$ is the volume of adsorption cell and $\rho_{r e c}$ is the density of residue gas. The adsorbate of charging cell is $m_{\text {char }}=V_{\text {char }} \rho_{\text {char }}$, where $V_{\text {char }}$ is the volume of charging cell and $\rho_{\text {char }}$ is the density of $\mathrm{CO}_{2}$ during charging. By turning on and off the "adsorbate gas introducing valve", a certain amount of $\mathrm{CO}_{2}$ was dosed into the adsorption cell for adsorption at the desired temperature. The thermal equilibrium was attained within 30 min to 1 hour. All time dependent adsorption data are collected from initial to final states. The residual mass left in the apparatus is $m_{\text {red }}=V_{\text {char }} \rho_{\text {char }}^{\prime}+V_{\text {rec }} \rho_{\text {rec }}^{\prime}$, where $\rho_{\text {char }}^{\prime}$ and $\rho_{\text {rec }}^{\prime}$ is the density of the residual $\mathrm{CO}_{2}$ in the charging and adsorption cells. Therefore, the subsequent adsorbed mass $\left(m_{a}\right)$ was calculated by the equation: $m_{a}=m_{r e c}+m_{c h a r}-m_{r e d}$. The amount of adsorbate uptake as a function of pressure and temperature was calculated as $q=m_{a} / M_{s}$, where $M_{s}$ is the mass of dry activated carbons. The $\mathrm{CO}_{2}$ density was calculated by indexing NIST Standard Reference Database 23 of Version 8.0 [144]. The limitations of the volumetric methods are (i) indirect measurement method, (ii) possibility of high errors (in that case, proper and high quality calibrations are needed), and (iii) time consuming, i.e., proper investigations are required during thermal equilibrium.

\subsubsection{Measurement of Water Uptakes on Silica Gels and AQSOA Zeolites}

The experimental investigations for water adsorption on zeolites samples were carried out by a thermogravimetric analyser. Figure 5.2 shows the schematic diagram for understanding the measurement of water vapour uptakes at various temperatures and pressures. At first, the zeolite sample was dried in an oven at $160^{\circ} \mathrm{C}$ for about 2 hours to remove all moisture from the sample. The zeolites were then placed on the sample cell, which was held by a microbalance, located 
above the adsorption chamber, known as the balance head. The balance head had to be set at a temperature at least $15^{\circ} \mathrm{C}$ above the experimental conditions to eliminate the condensation effects in the adsorption chamber. Dry Nitrogen (99.999\% purity) was introduced into the adsorption chamber to control buoyancy effects of the balance. The vapour was generated in a humidifier. The temperature inside the humidifier was measured by a class A RTD temperature sensor with an accuracy of $\pm 0.15{ }^{\circ} \mathrm{C}$. The vapour charging flow rate was controlled automatically. The adsorbent is placed in the quartz sample bowl inside the reaction chamber which has a radiant heater for maintaining a constant temperature. A vacuum pump was used for vacuuming and purging the system before an experiment and maintaining the adsorption chamber pressure in coordination with a vacuum controller.

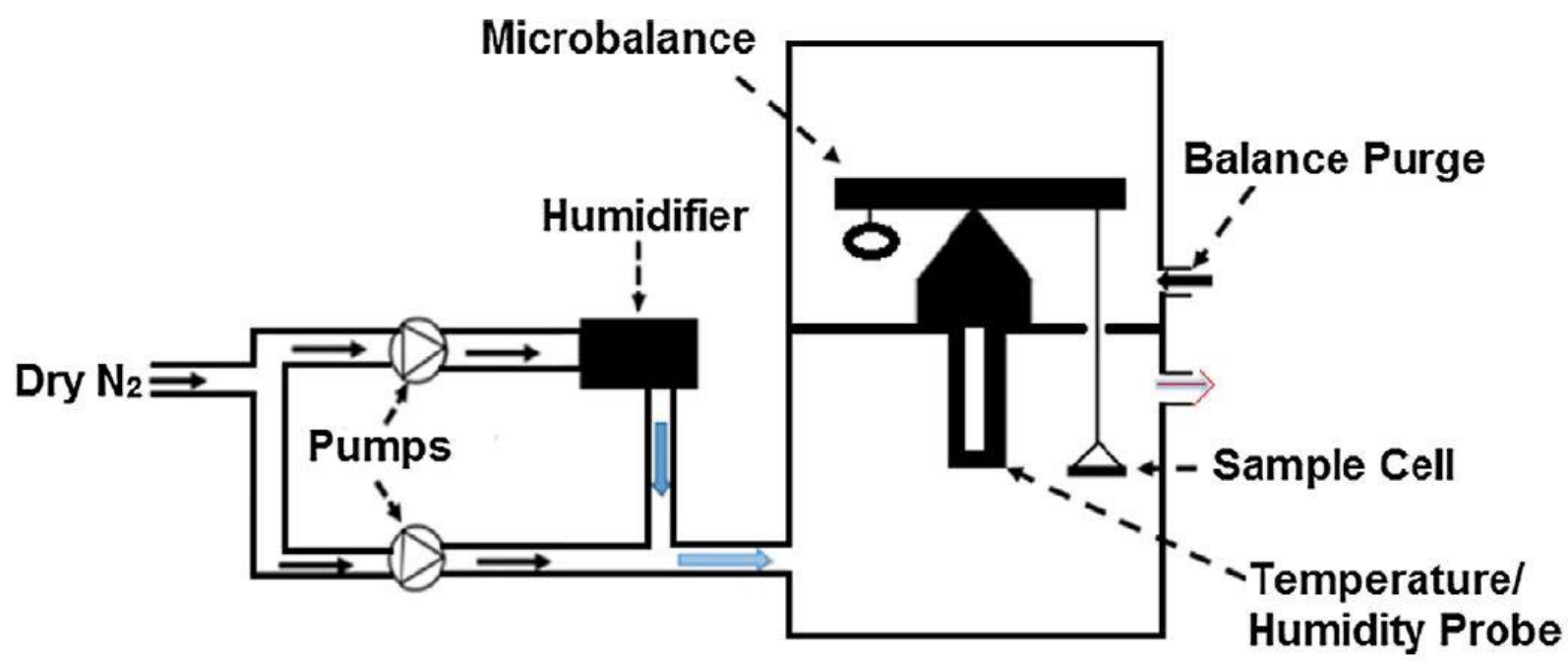

Figure 5.2 Schematic diagram of the adsorption/desorption isotherm and kinetics setup

During the adsorption experiment, both the pressure and temperature of the adsorption chamber were controlled within acceptable error ranges. Hence, the temperature probes and the humidity sensor were used to calculate the temperature and relative pressure (P/Ps). The humidity probe stabilises the value of P/Ps in the adsorption chamber by controlling the flow rate of dry $\mathrm{N}_{2}$. If $\mathrm{P} / \mathrm{Ps}$ is higher than that of expected values, the flow rates of dry $\mathrm{N}_{2}$ were increased and the $\mathrm{N}_{2}$ 
flow across the humidifier is stopped. The excess water vapour content was purged out of the chamber so that the P/Ps can be obtained at the desired value, which also introduces the right distribution of dry nitrogen to water vapour. If P/Ps was lower, the dry $\mathrm{N}_{2}$ flow through the humidifier was increased for the addition of water vapour content within the adsorption chamber. The water uptake was measured on the basis of P/Ps and the set temperature of the adsorption chamber. During the adsorption process, the amount of total mass, $\mathrm{M}_{\mathrm{t}}$, was increased due to the addition of water vapour from the humidifier. Subsequently, the $\mathrm{M}_{\mathrm{t}}$ of the adsorbent - adsorbate system is further increased as more water vapour is added to the chamber, making $\mathrm{M}_{t}$ a function of pressure. The mass of adsorbate, $m_{a}$, at any pressure $P_{i}$ can be determined by $m_{a, i}=M_{t}\left(P_{i}\right)-M_{t}\left(P_{o}\right)$, where $i$ is a variable to signify each pressure point, and $\mathrm{M}_{\mathrm{t}}\left(\mathrm{P}_{\mathrm{o}}\right)$ defines the total mass of zeolite + water vapour at initial known pressure $\mathrm{P}_{\mathrm{o}}$. The mass of adsorbate allows the gravimetric uptake to be calculated by $q_{i}=m_{a, i} / M_{s}$ for each pressure point, where $\mathrm{M}_{\mathrm{s}}$ is the mass of dry solid adsorbent. The adsorption kinetic data was also achieved while measuring the water uptake of the adsorbent as a function of time. Adsorption kinetic determines the amount of time taken for the adsorption to reach equilibrium at given temperature and pressure. Thus, at a specific temperature and pressure, the change in mass of adsorbent due to the increment of water vapour uptake was measured with respect to time.

For a given temperature, the $\mathrm{P} / \mathrm{P}_{\mathrm{s}}$ values were varied to attain the water uptakes to form the isotherm. Before achieving the new isotherm data, the adsorbents were retrieved from the adsorption chamber and placed in an oven at $150^{\circ} \mathrm{C}$ for regeneration. Then, with the regenerated adsorbent placed into the adsorption chamber, the adsorption chamber was purged with dry nitrogen at $65^{\circ} \mathrm{C}$. The experiment was repeatedly performed to ensure that the repeatability is within the experimental uncertainties. 


\subsection{Results and Discussions}

\subsection{1 $\mathrm{CO}_{2}$ Adsorption Isotherms}

The first two sets of data pertains to the adsorption of $\mathrm{CO}_{2}$ on pitch based activated carbon (type Maxsorb III) and activated carbon fibre (ACF type A-20) for the temperatures ranging from 303 $\mathrm{K}$ to $363 \mathrm{~K}$ and pressures up to $10 \mathrm{MPa}$, and these are shown in Figure 5.3 (a) and (b). The amount of $\mathrm{CO}_{2}$ uptakes on BPL and Norit activated carbons at 298, 308, 323 and $338 \mathrm{~K}$ and pressures up to $600 \mathrm{kPa}$ are observed to be of convex curvature of Type I, as shown in Figure 5.3 (c) and (d). Another set of data pertain to the adsorption of $\mathrm{CO}_{2}$ on monolith activated carbon. These are shown in Figure 5.3 (e). These experimental data can be predicted by adsorption isotherms equations namely Langmuir, Tóth and Dubinin-Astakhov (D-A). Hence, the Tóth isotherm equation fits well with experimentally measured adsorption isotherm data.

The Tóth isotherm model is presented by

$$
q=q^{o} \frac{K P}{\left\{1+(K P)^{n}\right\}^{1 / n}}
$$

where the Henry's constant is expressed as $K=K_{o} \exp \left(\frac{q_{s t}}{R T}\right)$. Here $K_{o}$ is the pre-exponential coefficient relating to the entropy of adsorption $(\Delta \mathrm{s}), R$ is the universal gas constant and $q^{o}$ indicates the limiting uptake [145]. The limiting uptake, $q^{o}$, is related to the adsorbent pore volume. The heterogeneity coefficient $n$ lies in the range 1 to 3 depending on the pore structures of adsorbents [146]. The Tóth isotherm equation can be further expressed as $P=\frac{1}{K}\left\{\frac{\left(q / q^{o}\right)^{n}}{1-\left(q / q^{o}\right)^{n}}\right\}^{\frac{1}{n}}$

The isosteric heat of adsorption is written as $q_{s t}=\left.R T^{2} \frac{\partial \ln P}{\partial T}\right|_{q}$. Substituting the values of $P$ in $q_{s t}$ equation, we have $q_{s t}=-R T^{2} \frac{\partial \ln K}{\partial T}$. The isotherms parameters are presented in Table 5.1 
Table 5.1 Adsorption isotherm parameters for various carbonaceous materials and $\mathrm{CO}_{2}$ system

\begin{tabular}{|c|c|c|c|c|c|}
\hline $\begin{array}{c}\text { Adsorbent }+\mathrm{CO}_{2} \\
\text { system }\end{array}$ & $\begin{array}{c}q_{s t} \\
(\mathrm{~kJ} / \mathrm{mol})\end{array}$ & $n$ & $\begin{array}{l}q^{o} \\
(\mathrm{~kg} / \mathrm{kg})\end{array}$ & $\begin{array}{c}K_{o} \\
\left(\mathrm{kPa}^{-1}\right)\end{array}$ & $\begin{array}{c}\text { Pore } \\
\text { volume } \\
\left(\mathrm{cm}^{3} / \mathrm{g}\right)\end{array}$ \\
\hline Maxsorb III $+\mathrm{CO}_{2}$ & 20.37 & 0.664 & 3.06 & $1.17 \times 10^{-7}$ & 1.70 \\
\hline $\mathrm{ACF}(\mathrm{A}-20)+\mathrm{CO}_{2}$ & 19.23 & 0.7 & 1.56 & $2.55 \times 10^{-7}$ & 1.05 \\
\hline $\begin{array}{c}\text { BPL activated } \\
\text { carbon }+\mathrm{CO}_{2}\end{array}$ & 25.00 & 1.2 & $\begin{array}{c}0.623- \\
0.001 \mathrm{~T}\end{array}$ & $1.10 \times 10^{-7}$ & 0.36 \\
\hline $\begin{array}{c}\text { Monolith activated } \\
\text { carbon }+\mathrm{CO}_{2}\end{array}$ & 24.02 & 1.0 & 0.25 & $6.20 \times 10^{-7}$ & 0.60 \\
\hline $\begin{array}{c}\text { Norit activated } \\
\text { carbon }+\mathrm{CO}_{2}\end{array}$ & 24.8 & 0.95 & 0.55 & $7.50 \times 10^{-8}$ & 0.47 \\
\hline
\end{tabular}



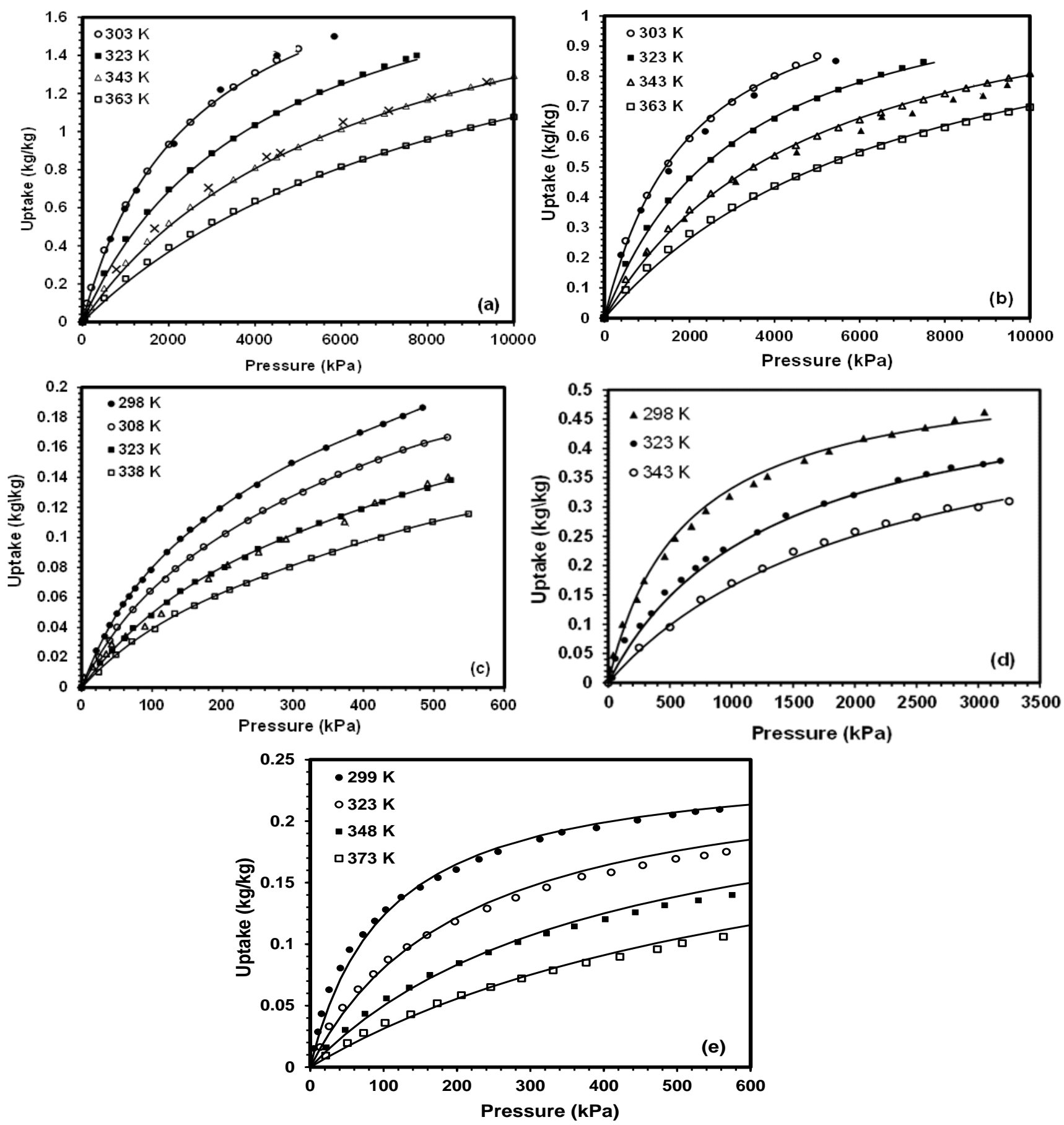

Figure 5.3 Adsorption isotherms diagrams for $\mathrm{CO}_{2}$ uptakes on (a) Maxsorb III (b) ACF-A20 (c) BPL (d) Norit and (e) honeycomb monolith type activated carbons for the temperatures ranging from $303 \mathrm{~K}$ to $373 \mathrm{~K}$ 


\subsubsection{Water Adsorption Isotherms}

In the present study, the temperatures and pressures investigated ranged from (303 to 338) K, and from (1 to 7) $\mathrm{kPa}$, respectively. The uncertainty of pressure ranges from ( \pm 10 to 200$) \mathrm{Pa}$. Emphasis is placed on the conditions experienced in the adsorber, since this is the range of conditions that directly determines the cooling capacity.

Adsorption equilibrium data of water vapour on three types of silica gels were obtained. Figure 5.4 (a), (b) and (c) depict the experimental equilibrium data for the type 'RD', 'A' and ' $3 \mathrm{~A}$ ' silica gels with water vapor, respectively.

The performance of type 'RD' silica gel water system employed by the adsorption chiller manufacturer available in the form of the Henry's isotherm is also superimposed for comparison [147]. The data of the manufacturer have been obtained for temperatures from 328 to $358 \mathrm{~K}$, and for pressures from 1 to $5 \mathrm{kPa}$. From Figure 5.4, it is found that the isotherms of $T=304 \mathrm{~K}$ and $T$ $=310 \mathrm{~K}$ for both type 'RD' and 'A' silica gel-water systems exhibit signs of onset of monolayer saturation at higher pressures. The Tóth's equation is found to fit the experimental data of both type 'RD' type 'A' and type ' $3 \mathrm{~A}$ ' silica gel-water systems well and is therefore used to find the isotherm parameters and isosteric heats of adsorption. The Toth isotherms parameters are shown in Table 5.2 
Table 5.2 Correlation coefficients for the two grades of Fuji Davison silica gel + water systems (The error quoted refers to the $95 \%$ confidence interval of the least square regression of the experimental data)

\begin{tabular}{|c|c|c|c|c|c|}
\hline Type & $\overline{K_{o}}$ & $\overline{q_{s t}}$ & $q^{o}$ & $\bar{t}$ & Remarks \\
\hline & $(\mathrm{kg} /(\mathrm{kg} \cdot \mathrm{kPa}))$ & $(\mathrm{kJ} / \mathrm{kg})$ & $(\mathrm{kg} / \mathrm{kg})$ & & \\
\hline 'A' & $(4.65 \pm 0.9) \cdot 10^{-10}$ & $(2.71 \pm 0.1) \cdot 10^{3}$ & 0.4 & 10 & By Tóth \\
\hline 'RD' & $(7.30 \pm 2) \cdot 10^{-10}$ & $(2.693 \pm 0.1) \cdot 10^{3}$ & 0.45 & 12 & By Tóth \\
\hline $\mathbf{3 A}$ & $(7.30 \pm 2) \cdot 10^{-10}$ & $(2.622 \pm 0.1) \cdot 10^{3}$ & 0.36 & 8 & By Tóth \\
\hline
\end{tabular}

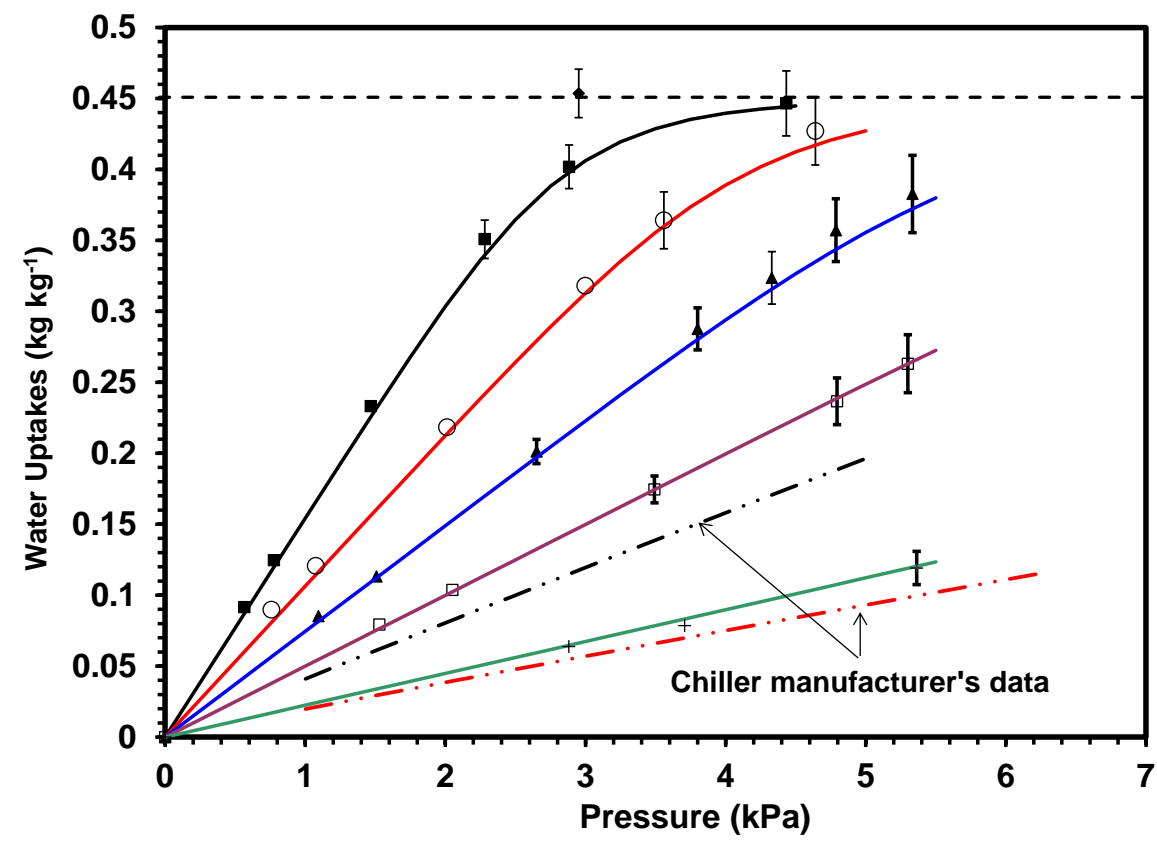

Figure 5.4 (a): Isotherm data for water vapour onto the type 'RD' silica gel; for experimental

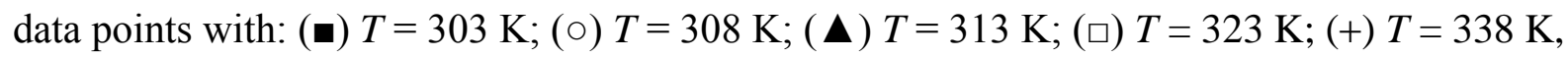
for computed data points with solid lines for the Tóth's equation. 


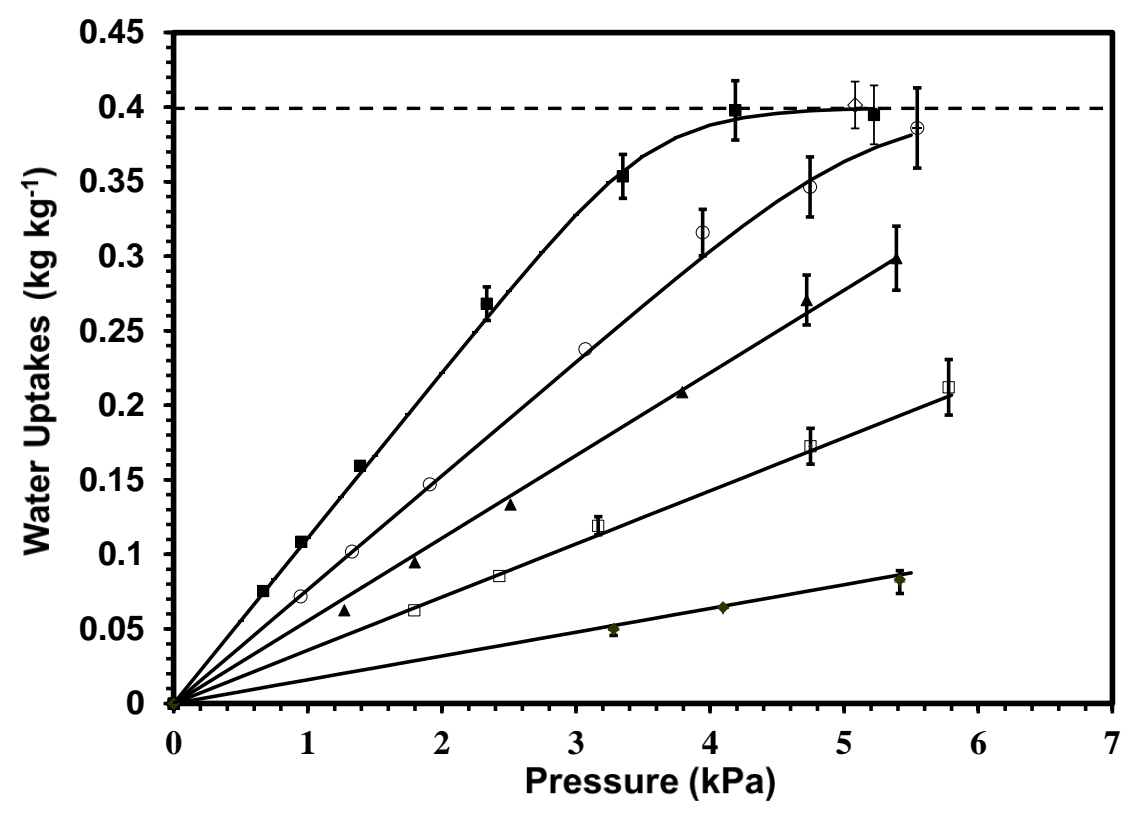

Figure 5.4 (b): Isotherm data for water vapour onto the type 'A' silica gel; for experimental data

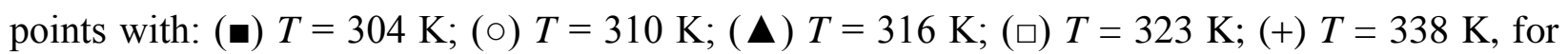
computed data points with solid lines for the Tóth's equation.

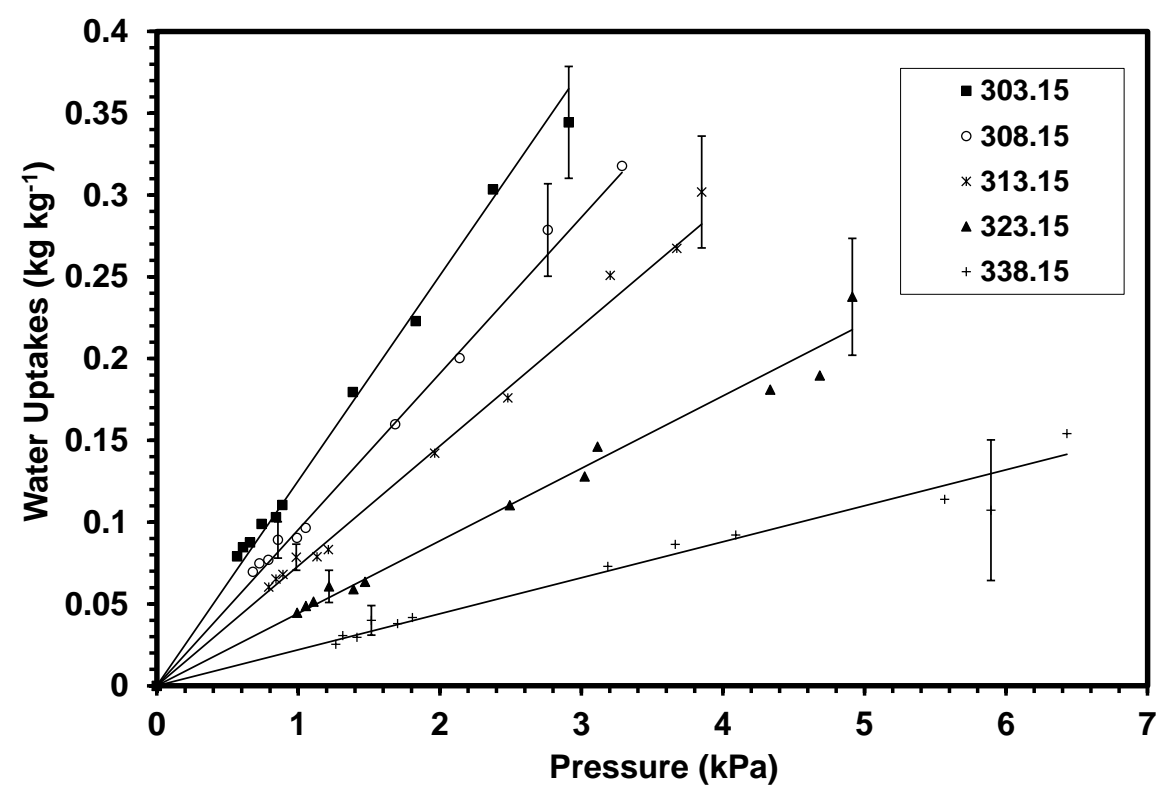

Figure 5.4 (c): Isotherm data for water vapor onto the type ' $3 A$ ' silica gel; for experimental data points with: $(\boldsymbol{\square}) \mathrm{T}=304 \mathrm{~K} ;(\mathrm{O}) \mathrm{T}=308 \mathrm{~K} ;\left(^{*}\right) \mathrm{T}=313 \mathrm{~K} ;(\boldsymbol{\Delta}) \mathrm{T}=323 \mathrm{~K} ;(+) \mathrm{T}=338 \mathrm{~K}$, for computed data points with solid lines for the Tóth's equation. 
Figure $5.5(\mathrm{a}-\mathrm{c})$ show the experimentally measured data for the adsorption of water vapour on AQSOA-Z01, Z02 and Z05 zeolites under static conditions. The experimental data of Shimooka et al. [116] at $298 \mathrm{~K}$ are included here for comparison purposes. From Figure 5.5 (a), the hydrophobic length of AQSOA-Z01 begins from Henry's region to the relative pressure (P/Ps) of 0.1. When P/Ps is higher than 0.1 , the water uptake increases sharply. This may be due to the fact that AQSOA-Z01 is mainly fabricated by $\mathrm{AlO}_{4}, \mathrm{PO}_{4}$ and $\mathrm{FeO}_{4}$ tetrahedron, and the $\mathrm{Al}$ and $\mathrm{P}$ atoms are partially replaced by $\mathrm{Fe}$ atoms. The $\mathrm{Fe}$ atoms attract more water molecules at higher pressure due to higher enthalpies of adsorption. On the other hand, Figure 5.5 (b) shows that AQSOA Z02 type zeolite has a very short hydrophobic length with water adsorption due to the presence of $\mathrm{SiO}_{4}$ tetrahedrons. With the presence of $\mathrm{SiO}_{4}$ in $\mathrm{AQSOA}-\mathrm{ZO} 2$ zeolite structure, water molecules are attracted towards this adsorbent at lower relative pressure as $\mathrm{Si}$ atoms has higher affinity with water. Similarly at the higher temperature of 333K, the isotherm also shows a short hydrophobic length at lower relative pressure. By considering the short hydrophobic length at lower pressure AQSOA-Z02 + water system provides S-shape or type V isotherm and indicates the presence of a pore filling phenomena. The isotherms at very low pressures are also shown in Figure 5.5 (b). Figure 5.5 (c) indicates that AQSOA-Z05 has longer hydrophobic length up to the relative pressure of 0.2 due to the absence of both Fe and Si atoms in AQSOA-Z05 structures. The crystal structure of $\mathrm{Z} 05$ consists mainly of $\mathrm{AlO}_{4}$ and $\mathrm{PO}_{4}$ tetrahedrons only. 

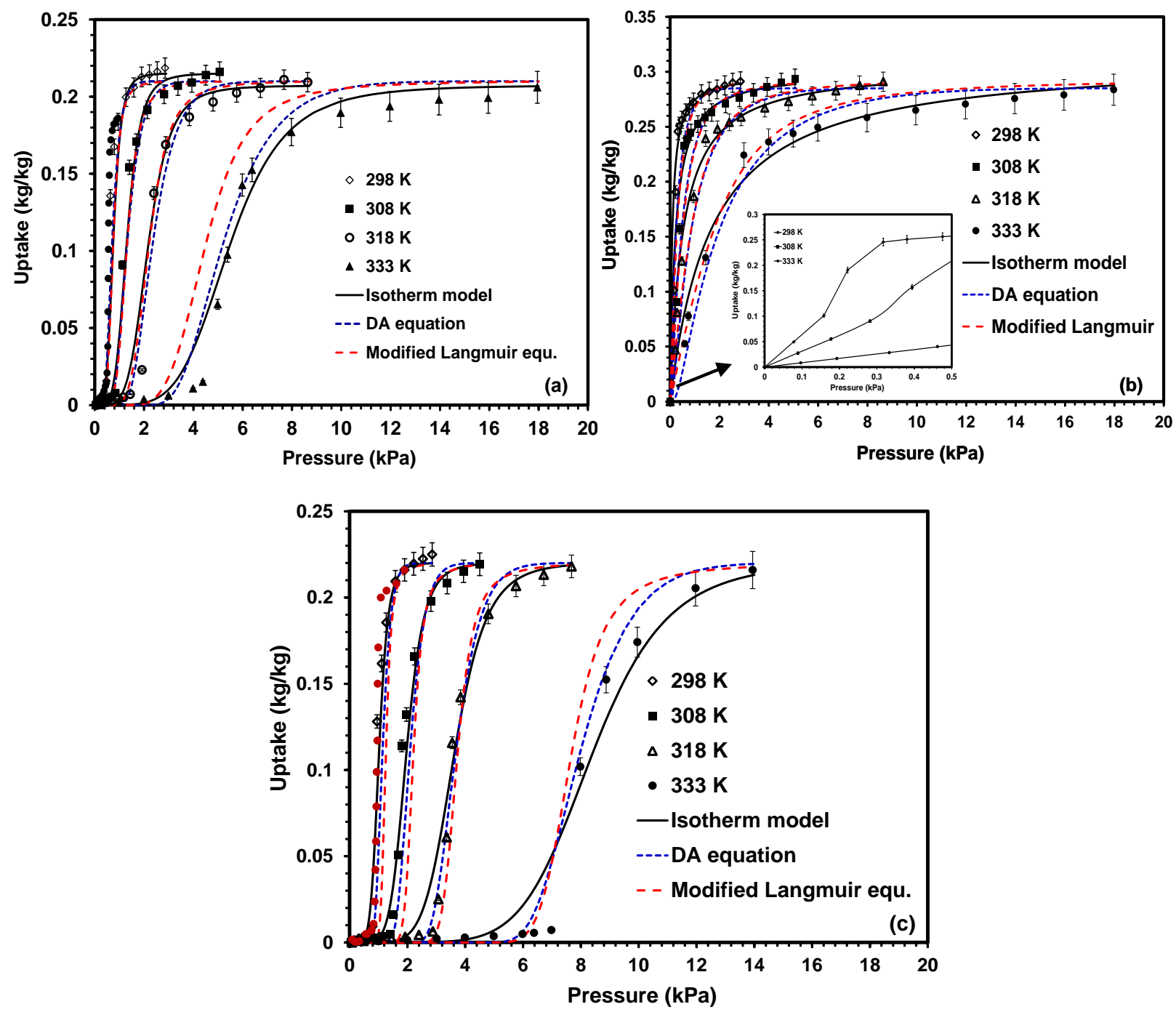

Figure 5.5 Experimentally measured water uptakes on (a) AQSOA-Z01, (b) AQSOA-Z02 and (c)

AQSOA-Z05 zeolites under static conditions.

Generally, Langmuir, Tóth and Dubinin-Astakhov equations fail to explain the S-shaped isotherm within acceptable error ranges [148]. Firstly, the Langmuir isotherm is derived based on two major assumptions that the adsorbent surface is homogeneous and the adsorptive sites are localized. These assumptions are not true for AQSOA zeolites. From the discussion of section 4.2.3 Interaction Potential of Zeolites with Water Molecule, we know that the most favourable adsorptive sites for water molecule adsorbed on AFI type zeolite are located in the centre of 
channels in the structure. Even for CHA type zeolite, the favourable adsorptive sites are located at the surface of the simulated structure. The CHA porous surface is not homogeneous. At low pressure region the water interaction on CHA zeolite is not very strong and shows the hydrophobic behaviours. However at relatively higher pressure regions, the water adsorption interaction is very high and the monolayer it is formed at the saturated pressure. The isotherm curve is not type I. Therefore, the Langmuir isotherm is not suitable for fitting adsorption data for AQSOA zeolite and water. Secondly, both Toth and DA isotherm are empirical equations and are generally valid for heterogeneous surface. Both DA and Toth (modified Langmuir) provide high errors with the experimental data of AQSOA-Zeolites and water systems. The adsorption isotherm model developed by Sun and Chakraborty [34] can almost cover the whole quadrant in a P (pressure)-q (uptake) coordinate system and therefore is adopted in the curve fitting of experimental data to reduce the fitting uncertainties.

$$
\theta=\frac{K\left(P / P_{s}\right)^{m}}{1+(K-1)\left(P / P_{s}\right)^{m}}
$$

where $K$ is the isotherm coefficient and is defined by $K=\alpha \exp \left[\mathrm{m}\left(q_{s t}{ }^{o}-h_{f g}\right)\right], \alpha$ is the preexponential coefficient, $m$ defines the surface-structural heterogeneity factor, $q_{s t}{ }^{o}$ is the isosteric heat of adsorption at zero surface coverage, $h_{f g}$ indicates the enthalpy of evaporation and $R$ is the gas constant. $\theta$ is the surface adsorbate coverage and is given by $q / q^{o}$, where $q$ is the amount of water vapour uptake and $q^{o}$ is the limiting uptake. Hence, $\left(q_{s t}{ }^{o}-h_{f g}\right)$ resembles the adsorption characteristics energy and can also be approximated from experimental data. Employing the concepts of Lennard - Jones and electrostatic potentials, the isosteric heat of adsorption at zero coverage $\left(q_{s t}{ }^{o}\right)$ is calculated as a function of pore width (as described in the previous chapter) or 


$$
q_{s t}^{o}=R T-\mathrm{N}_{\mathrm{A}} \frac{\int_{0}^{\mathrm{H}} \mathrm{V}_{\mathrm{ext}}(\mathrm{z}) \mathrm{e}^{\left(-\frac{\mathrm{v}_{\mathrm{ext}}(\mathrm{y})}{\mathrm{kT}}\right)} \mathrm{dz}}{\int_{0}^{\mathrm{H}} \mathrm{e}^{\left(-\frac{\mathrm{V}_{\text {ext }}(\mathrm{z})}{\mathrm{kT}}\right)} \mathrm{dz}}
$$

Hence $H_{c}$ indicates the pore width and $V_{e x t}$ is the adsorbent wall potential as derived by $V_{e x t}(z)=U_{s f}(z)+U_{s f}(H-z)$, where $H$ is the distance between the nuclei of the outer adsorbent atoms on opposite walls. The adsorbent-adsorbate interaction potential $\left(U_{s f}\right)$ as a function of pore width direction $(z)$ is defined as $U_{s f}=2 \pi \varepsilon_{s f} \rho_{s} \sigma_{s f}^{2} \Delta\left[\frac{2}{5}\left(\frac{\sigma_{s f}}{z}\right)^{10}-\left(\frac{\sigma_{s f}}{z}\right)^{4}-\frac{\sigma_{s f}^{4}}{3 \Delta(0.61 \Delta+z)^{3}}\right]$, where $\sigma_{s f}$ and $\varepsilon_{s f}$ are the solid-fluid collision diameter and the solid-fluid well depth potential, respectively. $\Delta$ defines the separation between the adsorbent planes and $\rho_{s}$ is the density of solid adsorbent. For water adsorption cases: $\sigma_{s f}=3.43 \AA$, $\varepsilon_{s f}=8.119 \times 10^{-21} \mathrm{~J} / \mathrm{mol}, \Delta=3.35 \AA$ and $\rho_{s}=$ 0.055 .

The experimentally measured water uptakes data are also fitted with Dubinin-Astakhov (DA) and the modified Langmuir equation to represent the characteristics curve. Mathematically, the DA equation is given by [5]

$$
q=q^{o} \exp \left[-\left\{\frac{R T}{E} \ln \left(\frac{P_{s}}{P}\right)\right\}^{n}\right]
$$

where $E$ defines the characteristics energy, $R$ is the gas constant, $P_{s}$ defines the saturated pressure and $n$ is the number of adsorptive sites or heterogeneity coefficient. The modified Langmuir adsorption isotherm equation is formulated on the basis of Fermi-Dirac distribution function, and it includes the loading, the adsorbent-adsorbate interaction and the surface structural heterogeneity factors [33]

$$
q=q^{o} \beta\left(\frac{P}{\varphi^{*}}\right) /\left[1+\left(\beta^{t_{1}}-\alpha\right)\left(\frac{P}{\varphi^{*}}\right)^{t_{1}}\right]^{\frac{1}{t_{1}}}
$$


where $\frac{P}{\varphi^{*}}=\left(\frac{P}{P_{s}}\right) \exp \left\{\frac{\phi_{m}}{R T}\left\{1-\left(\frac{P}{P_{s}}\right)^{n_{1}}\right\}+z\right\}$. Here $\phi_{m}$ is the minimum potential energy, $\beta$ is the loading factor, $t_{1}$ defines the surface heterogeneity, $n_{1}$ is the adsorbate-adsorbent interaction factor and $z$ indicates the compressibility factor.

Hence, the experimental data are fitted with the isotherm model of Equation (5.2) to calculated isotherm parameters for AQSOA zeolites + water systems. Figure 5.5 (a) indicates that the isotherm model is closely fitted with the experimental data within 5\% error margins both in the Henry and saturated pressure regions. However, the errors are higher than $8 \%$ for the relative pressure ranging from 0.1 to 0.3 . It is also found from Figure 5.5 (b) that Equation (5.2) shows a better curve-fitting (error within 5\%) for AQSOA-Z02 + water system as compared with other isotherms (Equations (5.4) and (5.5)). Figure 5.5 (c) indicates the S-shaped isotherm phenomenon, where the isotherm model of Equation (5.2) provides relatively higher errors (errors $>5 \%$ ) for the relative pressures varying from 0.1 to 0.3 . For water adsorption, the isosteric heat at zero coverage is calculated by Equation (5.3). According to equation (5.2), it should be noted here that $\alpha$ is related to the entropy of adsorption and that $m$ is responsible for the shape of the isotherm curve. From isotherm data, it is found that the hydrophobic length of AQSOA Z01 and Z05 depends on $m$ significantly. Hence the experimentally measured isotherm data are also fitted with Dubinin-Astakhov (DA) and the modified Langmuir equations. Both DA and Langmuir equations are able to handle the experimental data between $298 \mathrm{~K}$ and $318 \mathrm{~K}$ within experimental uncertainties as these equations are formulated from the rigour of micropore filling theory. However, DA and modified Langmuir equations do not work well at higher temperature adsorption. The parameters of DA and the modified Langmuir isotherm equations are also listed in Table 5.3 
Table 5.3 Parameters of isotherm equations for various AQSOA zeolites - water systems

\begin{tabular}{|c|c|c|c|c|c|c|c|}
\hline \multicolumn{8}{|c|}{ Isotherm equation } \\
\hline System & $\begin{array}{c}\mathrm{q}^{\mathrm{o}}(\mathrm{kg} \\
/ \mathrm{kg})\end{array}$ & \multicolumn{2}{|c|}{$q_{s t}{ }^{o}(\mathrm{~kJ} / \mathrm{kg})$} & \multicolumn{2}{|c|}{$m$} & \multicolumn{2}{|r|}{$\alpha$} \\
\hline AQSOA-Z01 + Water & 0.215 & \multicolumn{2}{|c|}{3030} & \multicolumn{2}{|c|}{4.9} & \multicolumn{2}{|r|}{$9.0 \times 10^{-7}$} \\
\hline AQSOA-Z02 + Water & 0.29 & \multicolumn{2}{|c|}{4560} & \multicolumn{2}{|c|}{1.01} & \multicolumn{2}{|r|}{$7.0 \times 10^{-6}$} \\
\hline AQSOA-Z05 + Water & 0.22 & \multicolumn{2}{|c|}{2800} & \multicolumn{2}{|c|}{6.55} & \multicolumn{2}{|r|}{$6.77 \times 10^{-5}$} \\
\hline \multicolumn{8}{|c|}{ Dubinin-Astakhov (DA) equation } \\
\hline System & \multicolumn{2}{|c|}{$\mathrm{q}^{\mathrm{o}}(\mathrm{kg} / \mathrm{kg})$} & \multicolumn{3}{|c|}{$\mathrm{E}(\mathrm{J} / \mathrm{mol})$} & \multicolumn{2}{|r|}{$\mathrm{n}$} \\
\hline AQSOA-Z01 + Water & \multirow{2}{*}{\multicolumn{2}{|c|}{0.21}} & \multicolumn{3}{|c|}{4000} & \multicolumn{2}{|r|}{5} \\
\hline AQSOA-Z02 + Water & & & \multirow{2}{*}{\multicolumn{3}{|c|}{$\begin{array}{l}7600 \\
2700\end{array}$}} & \multicolumn{2}{|r|}{2.9} \\
\hline AQSOA-Z05 + Water & 0.22 & $\begin{array}{c}0.285 \\
027\end{array}$ & & & & & 6 \\
\hline \multicolumn{8}{|c|}{ Modified Langmuir isotherm equation } \\
\hline System & $\mathrm{q}^{\mathrm{o}}(\mathrm{kg} / \mathrm{kg})$ & $\mathrm{z}$ & $\beta$ & $\alpha$ & $t_{1}$ & $\mathrm{n}_{1}$ & $\phi_{m}(\mathrm{~J} / \mathrm{mol})$ \\
\hline AQSOA-Z01 + Water & 0.21 & 3 & 0.6 & 0.002479 & 2 & -1.5 & 520 \\
\hline AQSOA-Z02 + Water & 0.29 & 2.6 & 0.5 & 0.005517 & 2 & 0.5 & 250 \\
\hline AQSOA-Z05 + Water & 0.22 & 3 & 1 & 0.011109 & 1.5 & -3.5 & 250 \\
\hline
\end{tabular}

\subsubsection{Summary}

This Chapter describes experimentally measured adsorption isotherms data employing both the volumetric and gravimetric method and these data are fitted with various isotherm equations. Based on isotherm data and the thermodynamic frameworks of adsorbate - adsorbent potentials, the next chapter develops a thermodynamic formulation from which the performance parameters of adsorption cooling and desalination system are calculated as a function of pore width and pore volume of adsorbent structure.

Employing isotherm data and isosteric heat as a function of pore width, the energetic performances of adsorption assisted cooling and desalination in terms of COP and SWP are calculated. The next chapter shows the thermodynamic frameworks of adsorption cooling and desalination, where the COP and SWP are shown in terms of pore width for activated carbons $\mathrm{CO}_{2}$, silica gel - water systems. 


\section{Chapter 6 Thermodynamic Frameworks for Adsorption Cooling}

\section{and Desalination Systems}

This chapter presents the thermodynamic frameworks for the calculation of coefficient of performance (COP), overall performance ratio (OPR) and the specific water production (SWP) as a function of pore width and volume of the adsorbent material. The performance parameters are calculated employing the formulations of isosteric heat at zero coverage and adsorbate uptake. Based on $\mathrm{CO}_{2}$ and water uptakes with the interaction potentials between various adsorbent adsorbate systems such as activated carbons $-\mathrm{CO}_{2}$, silica gel - water, the energetic performances are presented.

\subsection{Thermodynamic Framework of Adsorption Cooling and Desalination}

The adsorption chiller uses adsorbent - adsorbate characteristics and produces cooling effect at the evaporator by the amalgamation of adsorption-triggered-evaporation and desorptionactivated-condensation. This means that for continuous cooling purposes, the evaporated refrigerants are adsorbed into the adsorbent pores and results in the heat of adsorption. During desorption phase, the refrigerant, i.e., water vapour is desorbed (due to heat applied in the adsorber heat exchanger), and the desorbed water vapour is condensed in the condenser, and finally, the condenser water is refluxed back to the evaporator for the next cycle. On the other hand, the condensed water can be collected for desalination cycle, e.g., continuous production of desalinated water. It should be noted here that the adsorber (heat exchanger) is comprised of a copper tube - fin assembly where the porous adsorbents are housed with binders and stainless steel mesh. 
The schematic diagram of the two-bed adsorption cooling system with porous adsorbents (activated carbon for example) and adsorption bed or heat exchanger are shown in Figure 6.1 [10].

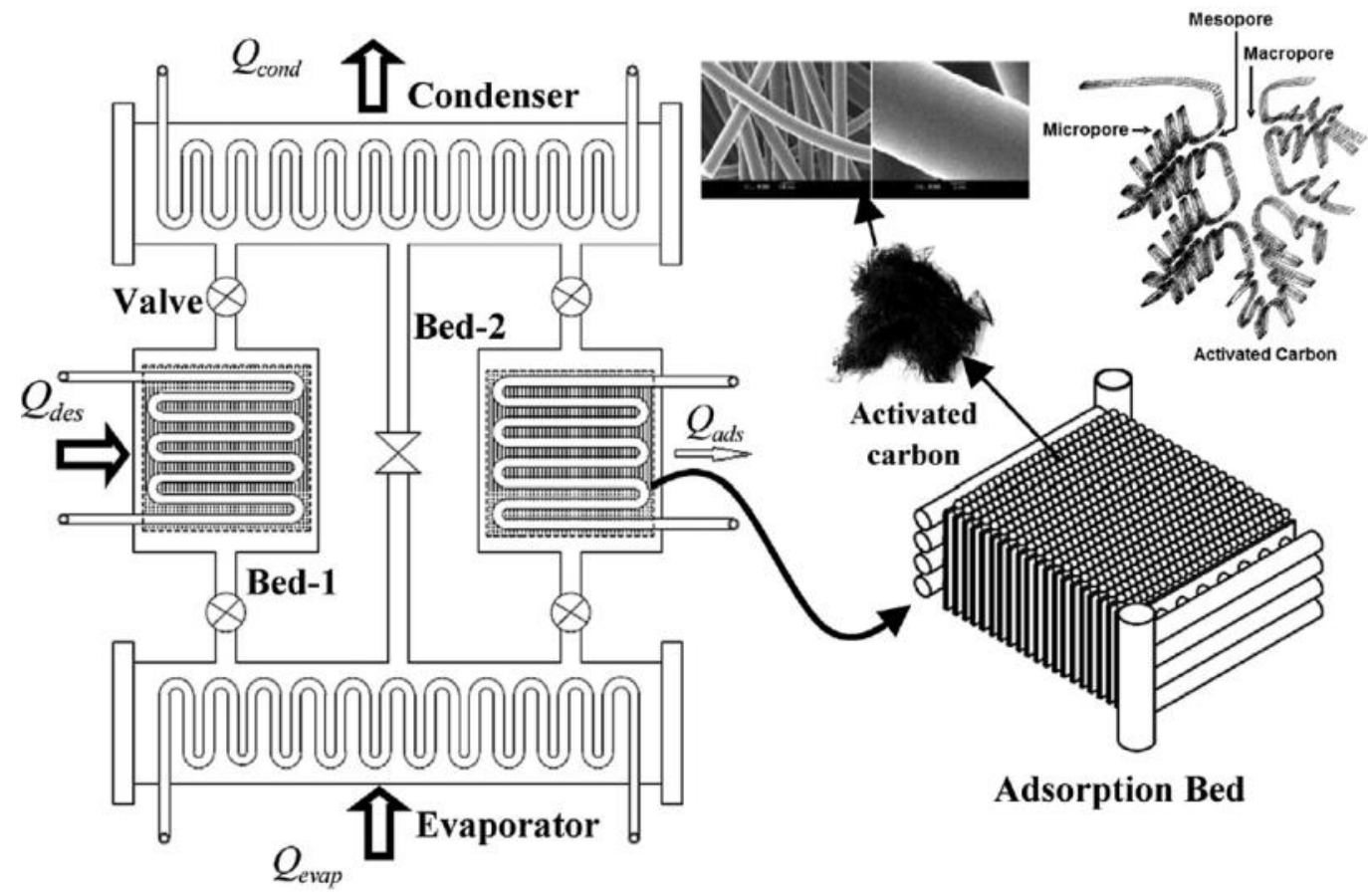

Figure 6.1 The schematic of adsorption chiller for continuous cooling

For explaining the mathematical modelling of adsorption assisted cooling and desalination, a general overview in pressure-temperature-uptake co-ordinate system is shown in Figure 6.2 


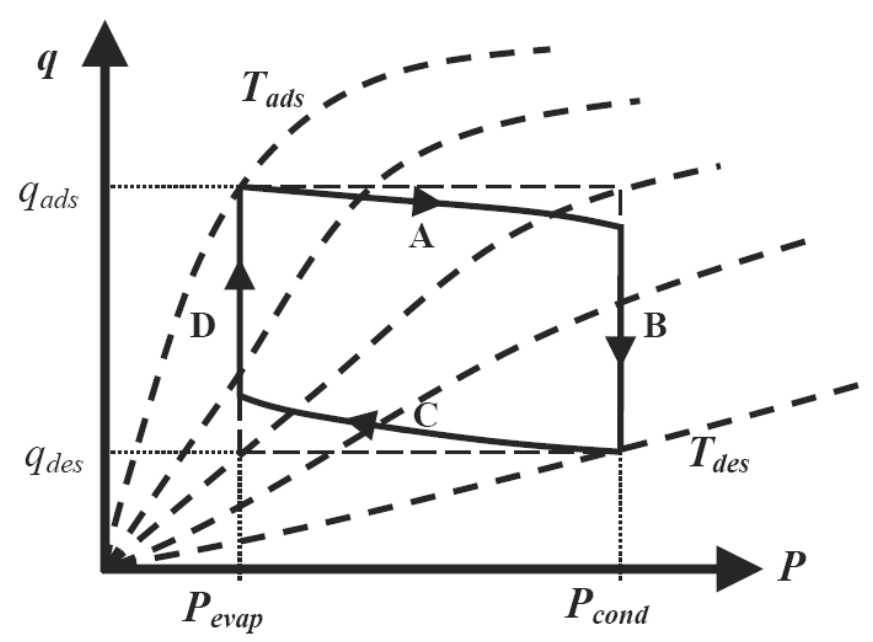

Figure 6.2 The schematic of adsorption - desorption processes in the adsorption bed employing pressure - temperature - uptakes co-ordinate system. Here the process A indicates the pressurisation in the adsorption bed with the effects of un-adsorbed adsorbate in void volume, B defines the flow out of high pressurised $\mathrm{CO}_{2}$ from the adsorption cell, the process $\mathrm{C}$ occurs due to the decrease of pressure in the adsorption bed and $\mathrm{D}$ indicates low pressure adsorbate flow into the sorption cell [10].

The amount of water uptake in terms of characteristics of adsorbent (e.g., pore width and volume) is given by [45],

$$
q=v_{p} \rho_{l} \exp \left[-\left\{\frac{\ln \left(P_{s} / P\right)}{1-\left(N_{A} \bar{V}_{\mathrm{ext}} / R T\right)-\left(q_{s t} / R T\right)}\right\}^{n}\right]
$$

where $v_{p}$ is pore volume, $\rho_{l}$ is liquid water density, $P_{s}$ is the saturation pressure at temperature and the Boltzmann average external wall potential is given by,

$$
\bar{V}_{\text {ext }}=\int_{0}^{H} V_{\text {ext }}(z) \exp \left\{-\frac{V_{\text {ext }}(z)}{k T}\right\} d z / \int_{0}^{H} \exp \left\{-\frac{V_{\text {ext }}(z)}{k T}\right\} d z
$$

By applying the adsorption, desorption, evaporation and condensation conditions to the equation, the water uptakes and offtakes during adsorption and desorption are calculated as, 


$$
\begin{aligned}
& q_{a d s}=v_{p} \rho_{l} \exp \left[-\left\{\frac{\ln \left(P_{s}\left(T_{\text {ads }}\right) / P_{\text {evap }}\right)}{1-\left(N_{A} \bar{V}_{\text {ext }} / R T_{\text {ads }}\right)-\left(q_{s t} / R T_{\text {ads }}\right)}\right\}^{n}\right] \\
& q_{\text {des }}=v_{p} \rho_{l} \exp \left[-\left\{\frac{\ln \left(P_{s}\left(T_{\text {des }}\right) / P_{\text {cond }}\right)}{1-\left(N_{A} \bar{V}_{\text {ext }} / R T_{\text {des }}\right)-\left(q_{s t} / R T_{\text {des }}\right)}\right\}^{n}\right]
\end{aligned}
$$

For continuous cooling operation, firstly a low-pressure refrigerant is evaporated at the evaporator due to external cooling load (or chilled water or cooling coil). The cooling capacity in $\mathrm{kW}\left(Q_{\text {evap }}\right)$ is calculated as,

$$
Q_{\text {evap }}=\int_{q_{\text {des }}}^{q_{\text {ads }}} h_{f g}\left(T_{\text {evap }}\right) d q-\int_{q_{\text {des }}}^{q_{\text {ads }}} \int_{T_{\text {evap }}}^{T_{\text {cond }}}\left(c_{p, l} d T\right) \mathrm{d} q-\int_{q_{\text {des }}}^{q_{\text {ads }}}\left\{h_{g}(P, T)-h_{g}(T)\right\} \mathrm{d} q
$$

The first term of the right-hand-side of the equation indicates the enthalpy of vaporization, the second term defines the amount of condensed heat that enters in to the evaporator, and the third term occurs due to the non-ideality of gaseous phase. Here the subscripts "ads" indicates the adsorption, "des" denotes desorption, "evap" is the evaporation and "cond" stands for the condensation. After evaporation, the refrigerant is adsorbed on the pores of solid adsorbent at relatively low pressure. Within the adsorption bed the heat of adsorption is released, which results in heating up the adsorbent and the adsorber heat exchanger. This heat of adsorption is removed by the cooling fluid during the adsorption phase. Secondly, the adsorbed vapour is released by supplying heating fluid to the pipes of sorption heat exchanger. As a result, the pressure and temperature of the adsorption bed increase up to $P_{\text {cond }}$ and $T_{\text {des }}$ (process $\mathrm{AB}$ of Figure 6.2), and the desorbed refrigerant enters into the condenser for condensation. During the desorption stage, the amount of heat $\left(Q_{\text {des }}\right)$ added to the sorption bed is calculated by [149], 


$$
\begin{aligned}
Q_{d e s}= & \int_{T_{a d s}}^{T_{\text {reg }}}\left[c_{p, s}+q_{a d s}\left\{c_{p, l}+q_{s t}\left(\frac{1}{T}-\frac{1}{v_{g}} \frac{d v_{g}}{d T}\right)\right\}\right] d T+\int_{q_{d e s}}^{q_{a d s}}\left\{h_{a}(P, T, q)-h_{g}(T)\right\} d q \\
& +\left.\int_{P_{\text {evap }}}^{P_{\text {cond }}} q_{s t} \frac{\partial q}{\partial P}\right|_{T} d P \\
& +\int_{T_{\text {reg }}}^{T_{\text {des }}}\left[c_{p, s}+q_{\text {des }}\left\{c_{p, l}+q_{s t}\left(\frac{1}{T}-\frac{1}{v_{g}} \frac{d v_{g}}{d T}\right)+\left.q_{s t} \frac{\partial q}{\partial T}\right|_{P}\right\}\right] d T
\end{aligned}
$$

where $c_{p}$ is the specific heat, $v$ is the specific volume and $h$ is the enthalpy. $T_{r e g}$ indicates the regeneration temperature at the beginning of desorption periods. The subscripts " $a$ ", " $s$ ", " $g$ " and "l" indicate adsorbate, solid, gaseous and liquid phases. The first term of the right hand side of Equation (6.6) indicates the sensible heat input per unit mass of adsorbent $\left(M_{s}\right)$ due to specific heat of adsorbent and its associate adsorbate for the temperatures ranging from $T_{a d s}$ to $T_{\text {reg }}$. The second terms indicate (i) the change in enthalpy with respect to the adsorbed and gaseous phases, and (ii) the isosteric heat due to change in pressures from $P_{\text {evap }}$ to $P_{\text {cond }}$. The last terms define sensible heats for the temperatures varying from $T_{r e g}$ to $T_{\text {des }}$.

The heat of condensation is given by,

$$
Q_{\text {cond }}=\int_{q_{\text {des }}}^{q_{a d s}} h_{f g}\left(T_{\text {cond }}\right) d q-\int_{q_{\text {des }}}^{q_{a d s}} \int_{T_{\text {evap }}}^{T_{\text {cond }}}\left(c_{p, l} d T\right) \mathrm{d} q+\int_{q_{\text {des }}}^{q_{\text {ads }}}\left\{h_{g}(P, T)-h_{g}(T)\right\} \mathrm{d} q
$$

The first term of the right hand side of the equation indicates the heat released by the condensed water, the second term is the amount of heat brought to evaporator from condenser and the last term occurs due to the non-ideality of gaseous phase.

Employing the thermodynamic property fields of an adsorbent-adsorbate system [149], the enthalpy of adsorbed phase is given by

$$
h_{a} \approx h(P, T, q=0)+\int_{T_{0}}^{T}\left[q_{s t}\left(\frac{1}{T}-\frac{1}{v_{g}} \frac{d v_{g}}{d T}\right)-\frac{\partial q_{s t}}{\partial T}\right] d T+\int_{0}^{q}\left(h_{g}-q_{s t}\right) d q
$$


It should be noted here that $\Delta q\left(=q_{a d s}-q_{d e s}\right)$ is the amount of $\mathrm{CO}_{2}$ refrigerant that can be delivered from the adsorption cell per adsorption-desorption cycle.

The performance parameter of adsorption cooling cycle is defined by the coefficient of performance (COP), i.e., $\left(Q_{\text {evap }} / Q_{\text {des }}\right)[10]$, where $Q_{\text {evap }}$ and $Q_{\text {des }}$ are cooling capacity and the amount of heat added to the sorption bed during desorption stage. The overall performance ratio (OPR) of the adsorption cycle, defined as the ratio of net useful effects (adsorption cooling or desalination) to the useful heat input, given by $\left.\left(Q_{\text {evap }}+Q_{\text {cond }}\right) / Q_{\text {des }}\right)[45]$.

For desalination, the same adsorption chiller is applied in the simulation and the only difference is that the condensed water will be collected from the condenser instead of flowing back to the evaporator in adsorption cooling cycles. The specific water production (SWP) is calculated as $\left(q_{a d s}-q_{d e s}\right)$.

\subsection{P-h (Pressure-Enthalpy) Diagram of Adsorption Assisted Cooling and}

\section{Desalination}

Employing Equation (6.3), the thermodynamic property surfaces of silica gel water system is plotted in a Pressure-enthalpy-uptake $(P-h-q)$ co-ordinate system as represented in Figure 6.3. 


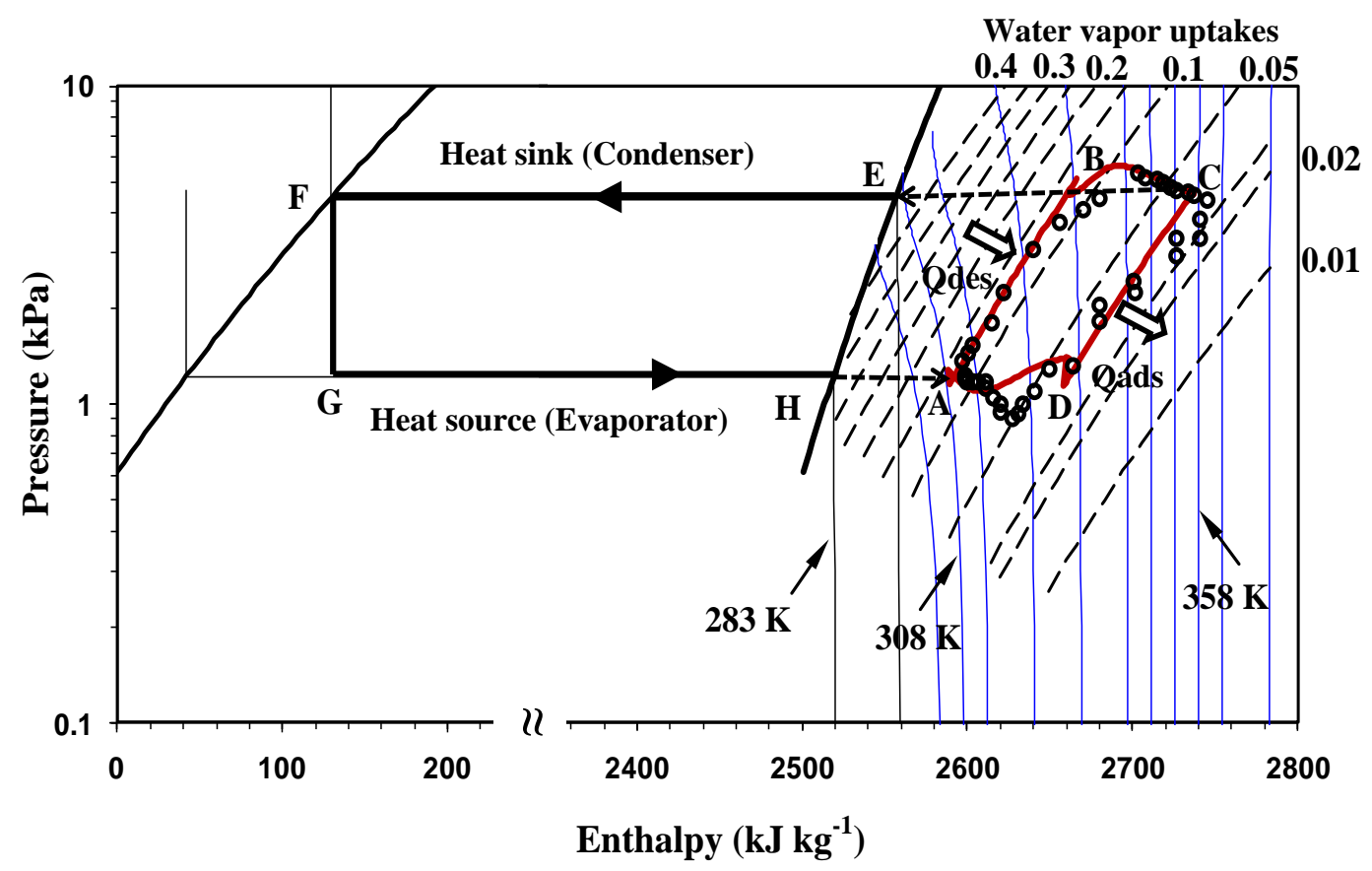

Figure 6.3 P-h diagram of ACCD cycle. Here "o" indicates our experimental data of type RD silica gel-water system.

From Figure 6.3, one can easily calculate the energetic performances of the adsorption cycle in terms of cooling capacity and water production. Due to the cooling load $\left(Q_{\text {evap }}\right)$, the enthalpy of evaporation $h_{f g}\left(=h_{H}-h_{G}\right)$ at the evaporator and the evaporated water vapor is adsorbed in silica gel surfaces of sorption bed (H-A). During the regeneration phase (lines A-B-C), the pressure in the adsorber rises from evaporator pressure $\left(P_{\text {evap }}\right)$ to condenser pressure $\left(P_{\text {cond }}\right)$ and the desorption of water vapour from silica gels occurs by connecting the adsorption bed with the condenser. The amount of uptake falls from $q_{a d s}$ to $q_{d e s}$, and the enthalpy of adsorbed phase changes from $h_{A}$ to $h_{C}$. At the condenser, the water vapour is condensed and heat is released to the environment. The amount of condensed water is calculated by $M_{s g}\left(q_{a d s}-q_{d e s}\right)$, where $M_{s g}$ is the mass of silica gel. During that phase, the enthalpy changes from $h_{C}$ to $h_{F}$. Finally the condensed water goes to the fresh water tank and completes the cycle. During the adsorption phase (lines C- 
D-A), the sorption bed is cooled and the pressure falls from $P_{\text {cond }}$ to $P_{\text {evap. }}$. The refrigerant vapour is adsorbed on the adsorbent and the amount of water is increased to $q_{\text {ads }}$.

\subsection{Performances of Silica Gel - Water based Cooling and Desalination}

For the silica gel water system, the Toth isotherm equation is employed to calculate the amount of water vapour uptakes and offtakes under adsorption chiller operating conditions. The maximum water uptakes are related to the adsorbent pore volume. The experimental data and the fitted isotherms modellings are provided in Chapter 5. The isosteric heats as a function of pore widths are also described in Chapter 4 in detail. In Chapter 3, it is observed that a rapid change in isosteric heat at zero coverage $\left(q_{s t}{ }^{o}\right)$ occurs for pore sizes smaller than $3 \AA$. For larger pores, there is a rapid decrease in $q_{s t}{ }^{o}$.

Figure 6.4 shows the COP as a function of pore width for various micropore volumes of silica gels. According to Figure 6.4, the $\mathrm{COP}$ is an increasing function of pore width up to the micro and mesopore ranges, and an increase of the total micropore volume $\left(v_{p}\right)$ leads to the improvement of the COP. This improvement is due to an increase of the mass of refrigerant exchanged during the adsorption cooling cycle, which indicates that the structural characteristics of silica gel affect the performances of adsorption cooling cycle. 


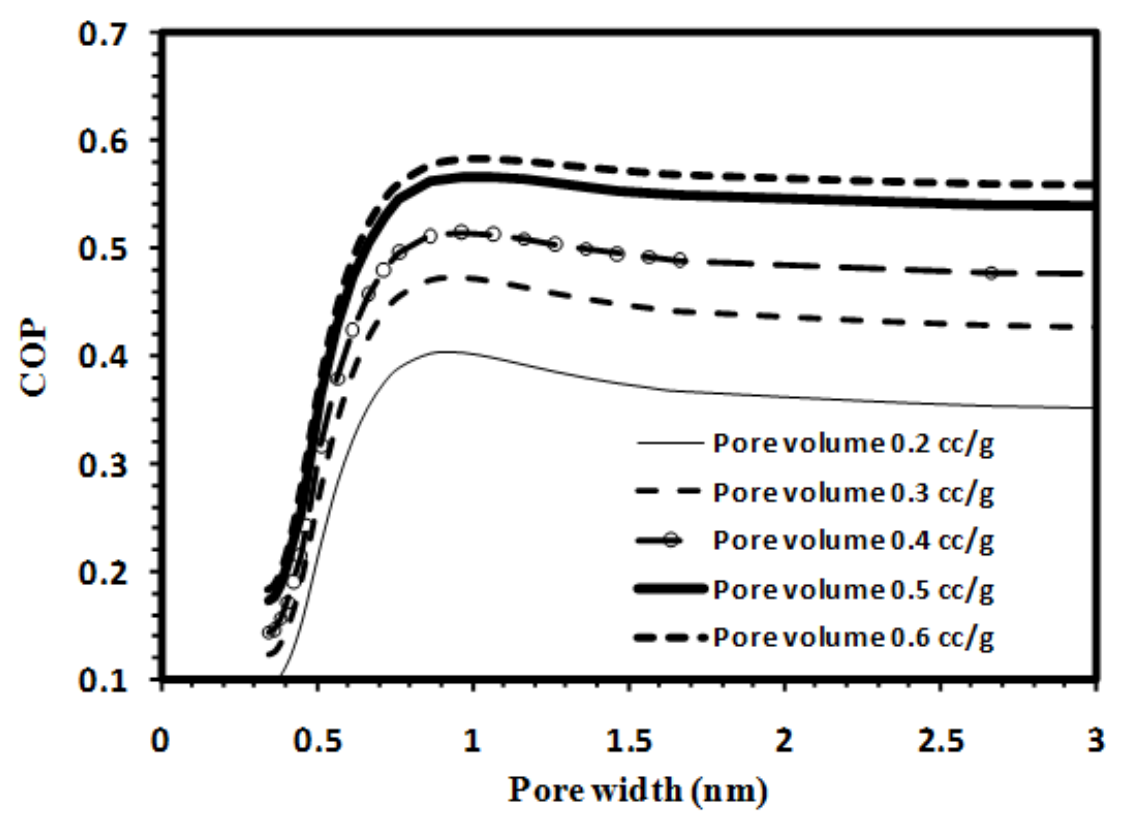

Figure 6.4 $\mathrm{COP}$ as a function of total micropore volume ranged from 0.3 to $0.6 \mathrm{cc} / \mathrm{g}$ and pore size for the regeneration temperature of $353 \mathrm{~K}$. Here cc indicates $\mathrm{cm}^{3}$.

Figure 6.5 shows the COP as a function of pore volume for various regeneration temperatures. An increase of the total micropore volume leads to an improvement of the COP due to an increase of the mass of water vapour exchanged during the cycle. The experimentally measured COP data as a function of regeneration temperatures are also plotted for comparisons. The influences of the pore width against the specific water production (SWP, $\mathrm{kg}$ of water per $\mathrm{kg}$ of silica gel) for various pore volumes and the evaporator temperature of $280 \mathrm{~K}$ are shown in Figure 6.6 It is found that the maximum amount of water is obtained for the pore widths ranging from $0.8 \mathrm{~nm}$ to $1 \mathrm{~nm}$. This is valid for all types of silica gels at the evaporator temperature varying from $278 \mathrm{~K}$ to ambient temperature. 


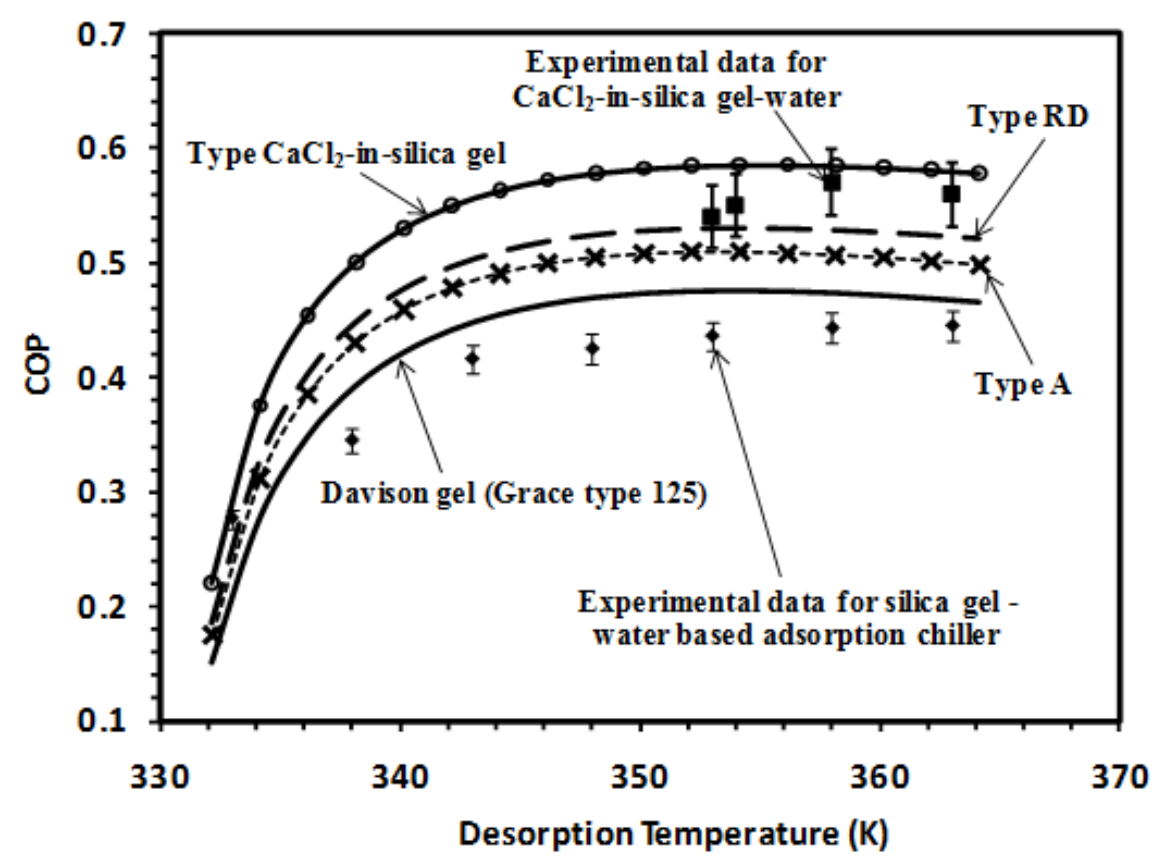

Figure 6.5 A plot of COP versus desorption temperature for various types of silica gels.

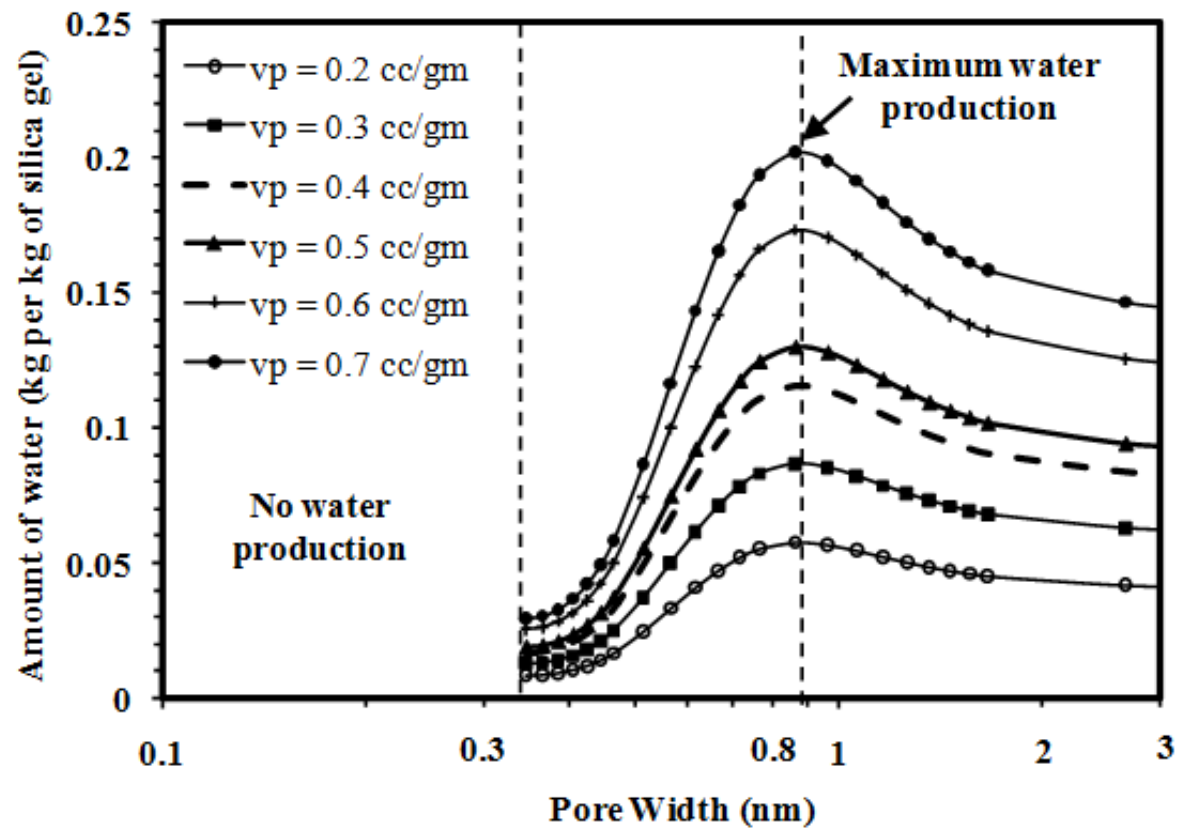

Figure 6.6 Influences of pore widths on water production for the evaporator temperature of 280 K. Pore volume is ranged from 0.2 to $0.7 \mathrm{cc} / \mathrm{g}$ and here $\mathrm{cc}$ indicates $\mathrm{cm}^{3}$. 
From literature, it is found that both 'RD' and 'A' types silica gels have similar pore size distribution curves and their local maxima are experimentally observed at $1.1 \mathrm{~nm}$ and $1.2 \mathrm{~nm}$, respectively. This means that the pore widths ranging from 0.8 to 1.2 provide the best performance in terms of COP and SWP (Figure 6.4 and Figure 6.6). It should also be noted here that in the meso-pore ranges (pore width $>2 \mathrm{~nm}$ ), the adsorption and desorption rates of water vapour on the silica gel adsorbent becomes slow and it takes longer cycles and switching times to complete the adsorption cycle, and the performances of the cooling and desalination drops.

The specific water production (SWP) for various heat source temperatures is shown in Figure 6.7 .

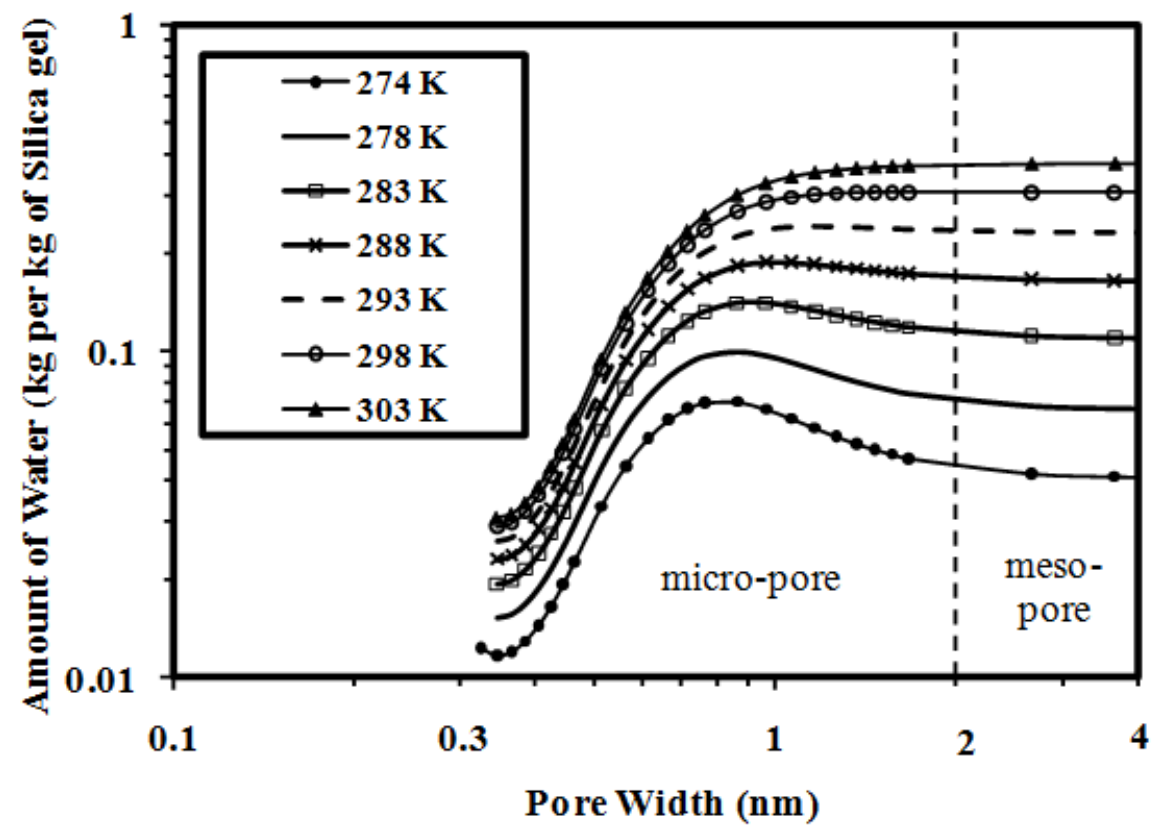

Figure 6.7 A plot of SWP against pore width for various evaporator temperatures. Here the evaporator temperatures range from $274 \mathrm{~K}$ to $303 \mathrm{~K}$ and the regeneration temperature is $353 \mathrm{~K}$. For evaporator temperatures ranging from $274 \mathrm{~K}$ to $288 \mathrm{~K}$, the maximum SWP is obtained for the pore width of $0.9 \mathrm{~nm}$. These variations occur due to the isosters moving as a function of isosteric heats. The SWP increases with higher evaporator temperature. 
The overall performance ratio (OPR) of the adsorption cycle, defined as the ratio of net useful effects to the useful heat input, is shown in Figure 6.8 As there are two useful effects namely the cooling and the desalinated water, a high OPR is expected. It could have a value of 1.1 at the evaporator temperature of $280 \mathrm{~K}$ (as a suitable temperature for cooling and desalination) for the pore width and volume of $0.9 \mathrm{~nm}$ and $0.45 \mathrm{~cm}^{3} / \mathrm{gm}$. This is valid for type RD silica gel.

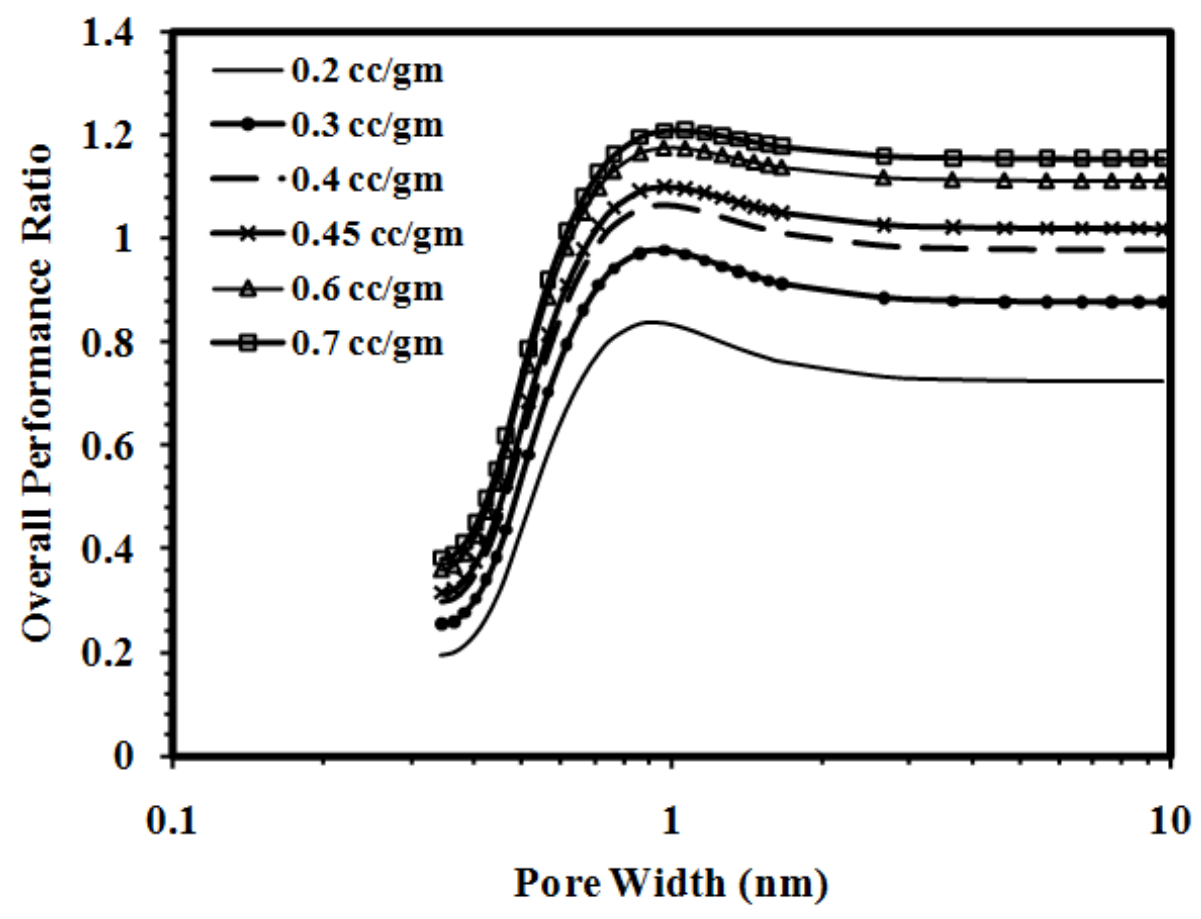

Figure 6.8 Overall performance ratio as a function of pore width for various pore volumes of silica gel. Pore volume is ranged from 0.2 to $0.7 \mathrm{cc} / \mathrm{g}$.

\subsection{Performances of Activated Carbons - $\mathrm{CO}_{2}$ based Cooling and Refrigeration}

In an adsorption cooling cycle, the relative pressure $\left(P / P_{s}\right)$ ranges from 0.1 to 0.2 which is a small portion of the full pressure range i.e. $P_{\text {evap }}$ and $P_{\text {cond }}$ are very small as compared with their saturation values. So the concept of isosteric heat at zero coverage as a function of pore widths can be applied to calculate the performances of adsorption chiller. Employing the relationship 
between the Henry's coefficient and the external adsorbent potential as a function of pore width direction $(y)$, the isosteric heat of adsorption at Henry's region is given by

$$
q_{s t}{ }^{o}=R T-\mathrm{N}_{\mathrm{A}} \frac{\int_{0}^{\mathrm{H}} \mathrm{V}_{\text {ext }}(\mathrm{z}) \mathrm{e}^{\left(-\frac{\mathrm{V}_{\text {ext }}(\mathrm{y})}{\mathrm{kT}}\right)} \mathrm{dz}}{\int_{0}^{\mathrm{H}} \mathrm{e}^{\left(-\frac{\mathrm{V}_{\text {ext }}(\mathrm{z})}{\mathrm{kT}}\right)} \mathrm{dz}}
$$

where $V_{\text {ext }}$ defines the average external potential of adsorbent + adsorbate (please refer to Chapter 3 for more detailed analysisr). The $\mathrm{V}_{\text {ext }}$ occurs due to the effects of adsorbent adsorbate and adsorbate - adsorbate interactions with the presence of coulomb forces. $k_{b}$ is the Boltzmann constant and $H_{c}$ is the pore width of solid adsorbents. The mathematical expression of $V_{e x t}$ for adsorbent - adsorbate interactions is given by

$$
V_{e x t}(y)=\phi_{s f}(y)+\phi_{s f}(H-y)
$$

Here $H\left(=H_{c}-\sigma_{s s}\right)$ is the pore width, $H_{c}$ is the distance between centres of carbon on opposing wall and $\sigma_{s s}$ is the size of carbon atom. The interactive potential between an adsorbate molecule and carbon wall $\left(\phi_{s f}\right)$ is written as [105]

$$
\phi_{s f}=2 \pi \varepsilon_{s f} \rho_{s} \sigma_{s f}^{2} \Delta\left[\frac{2}{5}\left(\frac{\sigma_{s f}}{y}\right)^{10}-\left(\frac{\sigma_{s f}}{y}\right)^{4}-\left(\frac{\sigma_{s f}^{4}}{3 \Delta(0.61 \Delta+y)^{3}}\right)\right]
$$

where $y$ is the distance of the adsorbate molecule to carbon wall surface, $\varepsilon_{s f}$ is the fluid-solid well depth, $\rho_{s}$ is the carbon density, $\sigma_{s f}$ is the fluid-solid collision diameter, $\Delta$ is the separation between carbon layer. The fluid-solid well depth $\varepsilon_{s f}$ and collision diameter $\sigma_{s f}$ are obtained using the Lorentz-Berthelot combining rules, i.e., $\varepsilon_{s f}=\sqrt{\varepsilon_{s s} \varepsilon_{f f}}$ and $\sigma_{s f}=\frac{\sigma_{s s}+\sigma_{f f}}{2}$, where $\varepsilon_{s s}$ is the well depth of carbon, $\varepsilon_{f f}$ is the well depth of adsorbate molecule (here $\mathrm{CO}_{2}$ ), $\sigma_{s s}$ is the 
size of carbon atom and $\sigma_{f f}$ is the size of $\mathrm{CO}_{2}$ molecule. The parameters used to calculate the isosteric heat of adsorption are furnished in Table 6.1

Table 6.1 Parameters of activated carbon and $\mathrm{CO}_{2}$ adsorbate molecules for simulation purposes

\begin{tabular}{|c|c|c|c|c|}
\hline \multicolumn{5}{|c|}{ Activated Carbon } \\
\hline$\Delta(\AA)$ & $\rho_{\mathrm{s}}\left(\AA^{-3}\right)$ & $\varepsilon_{s s} / k_{b}(\mathrm{~K})$ & $\sigma_{s s}(\AA)$ & References \\
\hline 3.35 & 0.114 & 28 & 3.4 & {$[150]$} \\
\hline \multicolumn{5}{|c|}{ Carbon Dioxide } \\
\hline Molecules & Group & $\varepsilon_{f f} / k_{b}(\mathrm{~K})$ & $\sigma_{f f}(\AA)$ & References \\
\hline $\mathrm{CO}_{2}$ & $\mathrm{C}$ & 28.129 & 3.49 & {$[151]$} \\
\cline { 2 - 5 } & $\mathrm{O}$ & 80.507 & 3.13 & {$[151]$} \\
\hline
\end{tabular}

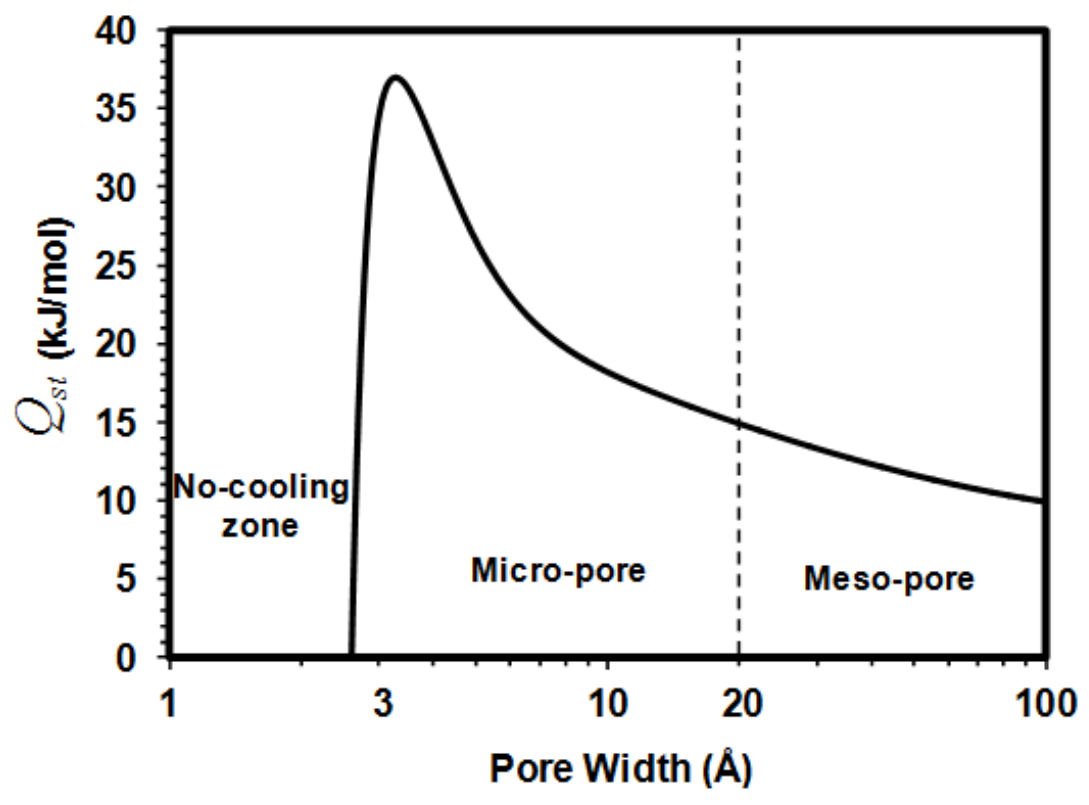

Figure 6.9 The plot of isosteric heat of adsorption as a function of pore width for the adsorption of $\mathrm{CO}_{2}$ on carbonaceous material

Here we consider mainly the effects of solid - fluid interactions to calculate the $q_{s t}{ }^{o}$. The plot of $q_{s t}{ }^{o}$ versus pore width is shown in Figure 6.9. It is observed that a rapid change in $q_{s t}{ }^{o}$ occurs for pore sizes that are smaller than the pore size at which the maximum $q_{s t}{ }^{o}$ is occurred due to the overlap of the force fields from opposite walls of the micropores. The value of $q_{s t}{ }^{o}$ decreases 
when the size of the pore increases i.e. in other words, for larger pores, there is a rapid decrease in $q_{s t}{ }^{\circ}$. In this section, the experimental isotherm data of activated carbon (type ACF-A20, Maxsorb III, BPL AC and monolithic carbon) and Toth isotherm parameters with $\mathrm{CO}_{2}$ as presented in Chapter 5 are used to highlight the present findings.

It should be noted here that the amount of $\mathrm{CO}_{2}$ uptakes and offtakes depend on the evaporator pressure and the condenser pressure with respect to the designed heat source and heat sink temperatures. The adsorption cooling cycle ranges from $P_{\text {evap }}$ to $P_{\text {cond }}$. The net amount of $\mathrm{CO}_{2}$ refrigerant per adsorption cooling cycle varies from $q_{a d s}$ and $q_{d e s}$. Here $q_{a d s}$ is measured as a function of $P_{\text {evap }}$ and $T_{a d s}$, where $T_{a d s}=31{ }^{\circ} \mathrm{C}$ and $P_{\text {evap }}$ is the saturation pressure of cooling load temperature that varies from $-5{ }^{\circ} \mathrm{C}$ to $15^{\circ} \mathrm{C}$. On the other hand, $q_{\text {des }}$ is calculated with respect to $P_{\text {cond }}$ and $T_{\text {des }}$, where $P_{\text {cond }}$ is the saturated pressure at $31^{\circ} \mathrm{C}$ and $\mathrm{T}_{\text {des }}$ ranges from 60 to $110{ }^{\circ} \mathrm{C}$.

The cooling effect is not achieved if the heating source temperature is considered as room temperature. For example, the minimum desorption temperature is $55{ }^{\circ} \mathrm{C}$ for the cooling load and heat sink temperatures of $10{ }^{\circ} \mathrm{C}$ and $31{ }^{\circ} \mathrm{C}$, respectively. The system performances (COP) against pore widths of various activated carbons are plotted in Figure 6.10. Hence the COP is presented for the heating source temperatures ranging from $60{ }^{\circ} \mathrm{C}$ to $110^{\circ} \mathrm{C}$. As can be seen from Figure 6.10(a) - (d), the COP is an increasing function of pore width up to (a) $10 \AA$ for Maxsorb III AC, (b) $11 \AA$ A for activated carbon fiber (A-20), (c) $8 \AA$ for BPL activated carbon, and (d) $15 \AA$ for the monolithic activated carbon. The COP decreases slightly at larger pore widths due to the adsorptive sites of decreasing energy. In the micropores, the amount of $\mathrm{CO}_{2}$ uptake increases due to higher force field in the adsorbent structure or in other words the mass of $\mathrm{CO}_{2}$ refrigerant per adsorption cooling cycle is also increased. Therefore the higher cooling capacity as well as the 
maximum COP is found in the micropore ranges. At super-micropore ranges ( $3 \AA$ to $5 \AA$ ), the COP drops significantly due to weak interactions between carbon and $\mathrm{CO}_{2}$ molecules.
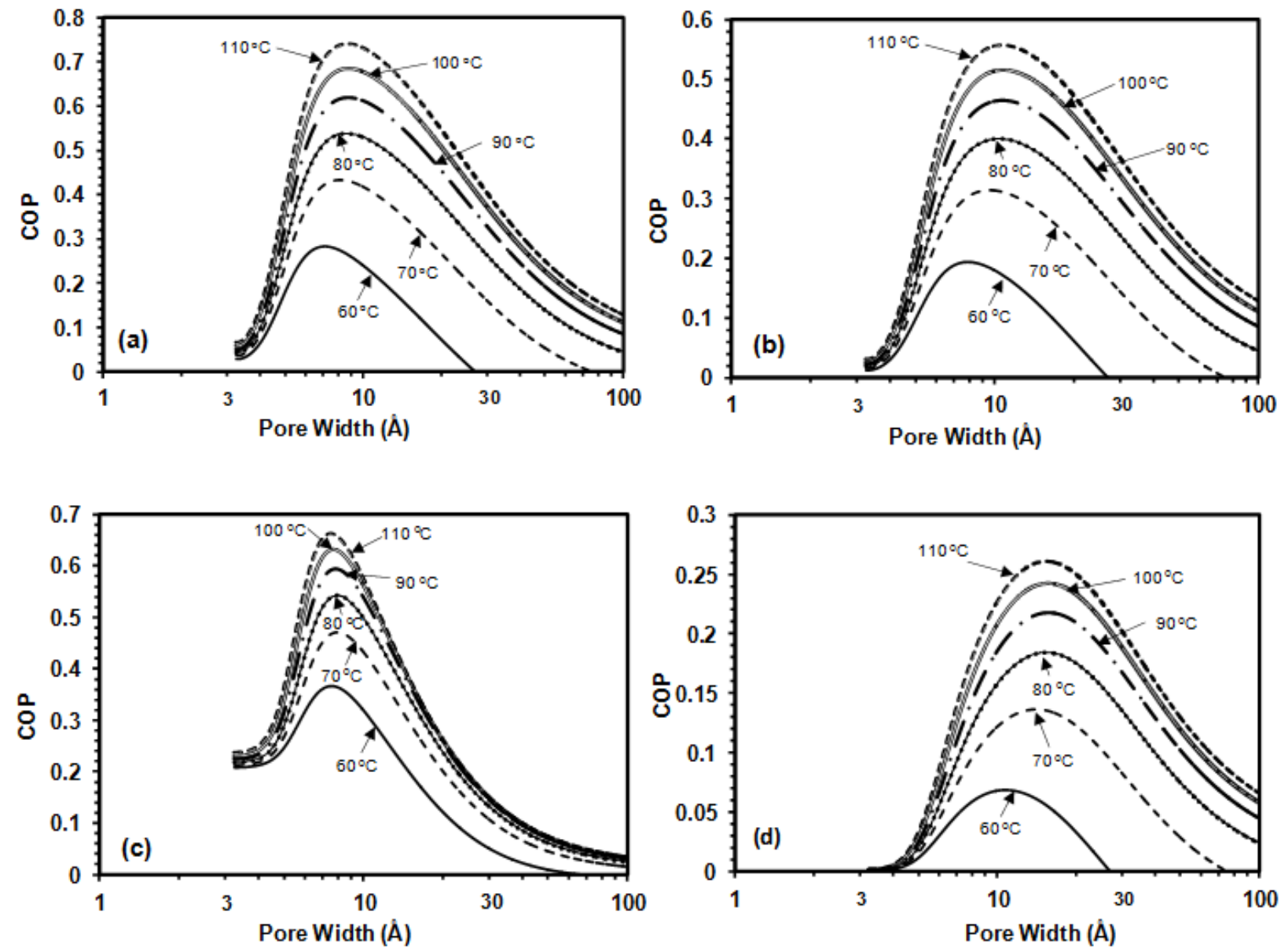

Figure 6.10 COP against pore widths for (a) pitch based activated carbon (Maxsorb III), (b) activated carbon fibre (ACF-A20), (c) BPL activated carbon and (d) monolith activated carbon based adsorption cooling cycle for the heat source temperatures ranging from $60^{\circ} \mathrm{C}$ to $110^{\circ} \mathrm{C}$ at the cooling load and the heat sink temperatures of $10^{\circ} \mathrm{C}$ and $30^{\circ} \mathrm{C}$, respectively. 

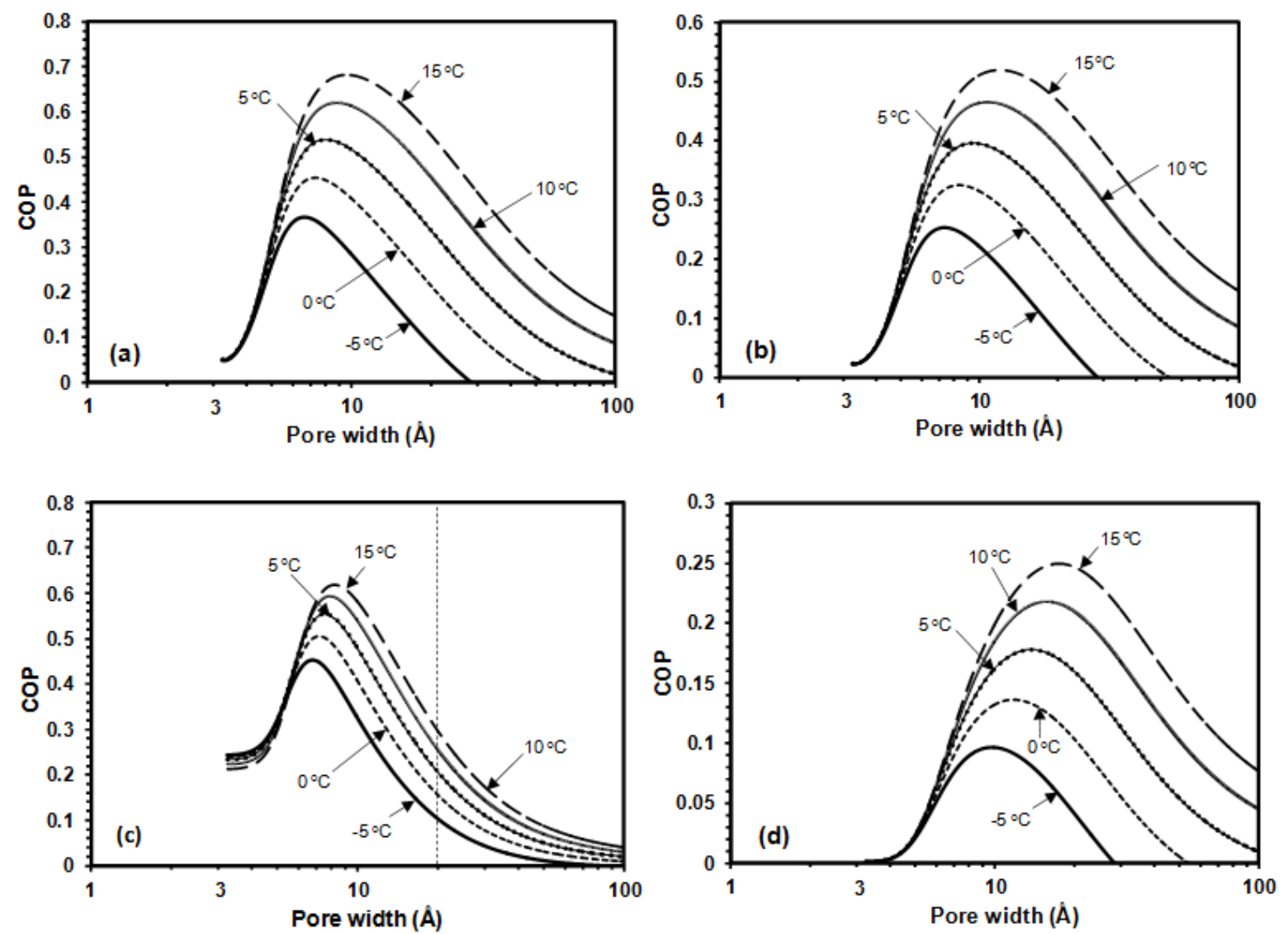

Figure 6.11 The COP of ADC as a function of pore widths at various cooling load temperatures for the adsorption of $\mathrm{CO}_{2}$ on (a) Maxsorb III, (b) ACF (A-20), (c) BPL activated carbon and (d) monolith activated carbon. Here the driving heat source and the heat sink temperatures are $90{ }^{\circ} \mathrm{C}$ and $10^{\circ} \mathrm{C}$, respectively.

Figure 6.11(a) - (d) show the COP against pore widths for the adsorption of $\mathrm{CO}_{2}$ on (a) Maxsorb III activated carbon (b) ACF (type A-20), (c) BPL AC and (d) monolithic AC for various cooling load temperatures at the heat source and the heat sink temperatures of $90^{\circ} \mathrm{C}$ and $10^{\circ} \mathrm{C}$, respectively. The COP increases at higher evaporator temperatures due to the transfer of more refrigerant per adsorption cooling period. No cooling effects are achieved for pore widths below $3 \AA$ as the $\mathrm{CO}_{2}$ molecules cannot penetrate into the pores of adsorbents during adsorption. The 
COP decreases gradually for larger pore widths. The adsorbate molecules are gradually accommodated in larger pores, in which the adsorption affinity becomes weaker. Therefore, a monotonic decrease in COP against pore widths is observed. The predictions for the maximum $\mathrm{COP}$ of adsorption cooling cycle employing carbonaceous materials and $\mathrm{CO}_{2}$ are shown in Table 6.2

Table 6.2 The optimum COP of activated carbons $+\mathrm{CO}_{2}$ assisted adsorption cooling cycle for various heat source temperatures at the cooling load and the heat sink temperatures of $10{ }^{\circ} \mathrm{C}$ and $30{ }^{\circ} \mathrm{C}$

\begin{tabular}{|l|c|c|c|c|c|c|c|}
\hline \multirow{2}{*}{$\begin{array}{l}\text { Adsorbent - adsorbate } \\
\text { system }\end{array}$} & Pore width $(\AA)$ & \multicolumn{5}{|c|}{ Average COP at various heat source temperatures } \\
\cline { 3 - 8 } & & $60{ }^{\circ} \mathrm{C}$ & $70{ }^{\circ} \mathrm{C}$ & $80{ }^{\circ} \mathrm{C}$ & $90{ }^{\circ} \mathrm{C}$ & $100{ }^{\circ} \mathrm{C}$ & $110{ }^{\circ} \mathrm{C}$ \\
\hline Maxsorb III $-\mathrm{CO}_{2}$ & $7 \sim 15 \AA$ & 0.28 & 0.40 & 0.51 & 0.60 & 0.67 & 0.73 \\
\hline $\mathrm{ACF}(\mathrm{A}-20)-\mathrm{CO}_{2}$ & $8 \sim 12 \AA$ & 0.19 & 0.30 & 0.40 & 0.46 & 0.51 & 0.55 \\
\hline $\mathrm{BPL} \mathrm{AC}-\mathrm{CO}_{2}$ & $7 \sim 10 \AA$ & 0.33 & 0.45 & 0.53 & 0.58 & 0.62 & 0.65 \\
\hline Monolith $\mathrm{AC}-\mathrm{CO}_{2}$ & $10 \sim 20 \AA$ & 0.06 & 0.13 & 0.18 & 0.21 & 0.24 & 0.26 \\
\hline
\end{tabular}

It is found that the COP is an increasing function of heating source temperatures. The pore width generally ranges from 7 to $15 \AA$ for maximum COP. Most carbonaceous adsorbents such as Maxsorb III, ACF, BPL AC and monolith AC consist of micro, meso and macropores. Both Maxsorb III and ACF possess more micro adsorptive sites where the overlapping interactions of $\mathrm{CO}_{2}$ molecules increase during adsorption. On the other hand, the BPL activated carbon contains comparatively less adsorption sites with small pore volume. Therefore a sharp decrease in COP occurs in the meso and macro pores. The pore size of monolith AC ranges from $5 \AA$ to $200 \AA$ [152] and it shows relatively lower COP due to smaller pore volume with larger pore sizes. For monolith AC, the optimum COP is found at $15 \AA$. There are low or no cooling effects at the macro-pore zones or the pore width below $3 \AA$. 


\subsection{Summary}

From the present analysis, it is noticed that silica gels optimised with respect to pore sizes and volumes for a given evaporating temperature can entail an increase of the COP and OPR of the order of 20 to $40 \%$, which cannot be neglected. If the evaporator temperature varies and especially when it decreases, there is a decreasing tendency of COP. Another conclusion of this study is that an increase of pore volume does not entail systematically an improvement of performance. The best performance of adsorption cooling cycle is obtained from a compromise between the greatest pore volume and the optimal pore size. From the present analysis, it is observed that there is no cooling and water production performance for pore widths varying from 0 to $0.3 \mathrm{~nm}$. For better efficiency, the silica gel should be designed with the pore size of $2 \mathrm{~nm}$. For cooling and refrigeration purposes, the carbonaceous materials should be designed with micro-pores ranging from 7 to $15 \AA$ and higher micro-pore volume. The knowledge of isosteric heat at zero coverage for an adsorbent - adsorbate system can help in the design of new carbonaceous materials such as carbon nanotubes, carbon fibres, pitch based activated carbon, metal organic frameworks, and carbon-silica composites. From the present analysis, we can approximately find the optimum ranges of pore widths that would be desirable to design porous activated carbons for the adsorption of carbon dioxide on carbon materials in refrigeration and cooling applications. The overall conclusions and future directions are presented in the following chapter. 


\section{Chapter 7 Conclusions and Future Work}

\subsection{Conclusions}

In this thesis, a thermodynamic framework for calculating the isosteric heat of adsorption at Henry's region has been derived and developed for various adsorbent + adsorbate systems. The construction of adsorbent molecular structure and adsorbates with various orientations were achieved at first. Secondly, the pore models based on the molecular structures of adsorbent have been proposed. Thirdly, the total interaction potential between adsorbent and adsorbate are computed. Finally, the isosteric heat of adsorption at zero coverage in terms of adsorbent adsorbate interactions and pore width, is calculated and compared with experimentally measured data.

Employing the proposed thermodynamic frameworks, the connections between the isosteric heat and the parameters such as pore width of adsorbent structure have been established. Finally, the thermodynamic performances such as COP and OPR are evaluated for adsorption assisted cooling and desalination systems.

The thermodynamic framework is built and applied to adsorbent materials of various structures. Firstly, the graphite structure is considered as simple layers of lattice with parallel slit-shaped pore model [14]. The total interaction potential between graphite and polar molecules (such as water, methanol and ethanol) and nonpolar molecules [10] (such as $\mathrm{CO}_{2}$ ) are obtained employing the reduced energy expansion equation $[107,153]$ for polar molecules with various orientations. The isosteric heat of adsorption is derived with respect to the pressure-uptake-temperature coordinate system [13]. It is found that he maximum potential well for graphite - water system constitutes $91.28 \%$ Lennard Jones potential vLJ $\left(\mathrm{C} \cdots \cdot \mathrm{H}_{2} \mathrm{O}\right), 8.7 \%$ induction potential $\mathrm{vI}\left(\mathrm{C} \cdot \cdots \cdot \mathrm{H}_{2} \mathrm{O}\right)$ and $0.012 \%$ electrostatic $\mathrm{vE}\left(\mathrm{C} \cdots \cdot \mathrm{H}_{2} \mathrm{O}\right)$. The maximum potential well depth depends on various 
orientations of $\mathrm{H}_{2} \mathrm{O}$ molecule on graphite structure. The maximum $q_{s t}^{o}$ at zero surface coverage is found to be $0.35 \mathrm{eV}$ at orientation 1, where the oxygen atom is at the origin, one $\mathrm{H}$ atom is at the $\mathrm{X}$-axis and $\mathrm{H}-\mathrm{O}-\mathrm{H}$ is obtained in the $\mathrm{X}-\mathrm{Y}$ plane, and the simulation result is very close to the experimental data of $0.32 \mathrm{eV}$. For water molecules, the electrostatic contribution for $\mathrm{v}(\mathrm{z})$ is very close to zero and provides attractive or repulsive interactions between adsorbate and graphite molecules. For ethanol and methanol, the electrostatic contribution is close to zero and provides both attractive and repulsive interactions. The $\operatorname{vLJ}\left(\mathrm{C} \cdots \mathrm{CH}_{3}-\mathrm{OH}\right)$ and $\operatorname{vLJ}\left(\mathrm{C} \cdots \mathrm{CH}_{3}-\mathrm{CH}_{2}-\mathrm{OH}\right)$ contributions are more than $90 \%$. The induction interactions are less than $10 \%$. For both methanol and ethanol molecules, the induction potential increases exponentially for the separation distance decreasing from $3 \AA$ to 0 . However, the electrostatic potential remains constant and the value is very close to zero.

Secondly, the amorphous silica structure is constructed [110] by the Reverse Monte Carlo (RMC) [99] simulation tool with experimentally measured X-ray Diffraction (XRD) data of silica sample [109]. The crystallographic properties including partial and total radial distribution and bond angles of the constructed silica structure are compared to experimental results. For simplicity, the simple slit-shaped pore model and Clausius - Clapeyron equation are applied to calculate the isosteric heat of adsorption of water on silica surface. It is found that the partial radial distribution and structure factor of the reference silica structure constructed by Reverse Monte Carlo method are calculated to confirm the validity of $\mathrm{SiO}_{2}$ structure for $q_{s t}{ }^{o}$ calculation. Furthermore, the bond angle (O-Si-O and $\mathrm{Si}-\mathrm{O}-\mathrm{Si})$ distribution functions of the silica structure are calculated. The O-Si-O and Si-O-Si angles are mainly around $104^{\circ} \sim 112^{\circ}$ and $130^{\circ} \sim 145^{\circ}$ and are in good agreement with other simulation results while the most probable bond angle (MPBA) of $\mathrm{Si}-\mathrm{O}-\mathrm{Si}$ is found to be $146^{\circ}$ and that of $\mathrm{O}-\mathrm{Si}-\mathrm{O}$ is $109.4^{\circ}$ [112]. The interaction 
potential is also determined by the water-silica distance $\mathrm{z}$ according to the interaction potential of five positions in orientation 1, which are chosen in the simulation box due to the fact that the centre $\mathrm{x}=-11.5 \AA$ and $\mathrm{y}=-11.5 \AA$ is a favorable adsorptive site due to the presence of the negative interaction potential. While the $\mathrm{x}$ coordinate is varied from $-11 \AA$ to $-12 \AA$ and $\mathrm{y}$ coordinate is remained fixed at $-11.5 \AA$, the maximum interaction potential ranges from -0.782 $\mathrm{eV}$ to $-0.677 \mathrm{eV}$.

Thirdly, the molecular structures of three types of zeolites (AQSOA-Z01, Z02 and Z05 [115117]) are generally considered as cylindrical channel frameworks. The interaction potential between water molecule and the surfaces of these channels are calculated employing the cylindrical interaction potential equation [118-120] with two zeotype parameters (AFI type for Z01 and Z05, CHA type for Z02). The isosteric heat of adsorption of water on AQSOA-Z01, Z02 and Z05 zeolites are calculated employing the modified Clausius - Clapeyron for cylindrical pore model. The most favourable adsorptive sites with negatively larger potentials are identified at the positions of channels inside the zeolite framework. The isosteric heat of adsorption for water adsorption on CHA and AFI types zeolites are predicted to be (a) $50 \mathrm{~kJ} / \mathrm{mol}$ and $80 \mathrm{~kJ} / \mathrm{mol}$ respectively employing Steele's interaction potential equation, and (b) $36.26 \mathrm{~kJ} / \mathrm{mol}$ and $51.4 \mathrm{~kJ}$ /mol respectively employing cylindrical interaction potential equation.

All of the theoretically derived isosteric heats are compared with experimental data in the literature. Particularly, the isosteric heats are derived from our experimentally measured adsorption isotherms of calorimetric analysis.

In this thesis, the porous properties of various activated carbons are measured in terms of pore size, pore volume and material structures to understand the nature of these activated carbon materials. A volumetric method is employed to measure the amount of $\mathrm{CO}_{2}$ uptakes on various 
activated carbons. The Tóth isotherm model is fitted with the experimental data within the acceptable error ranges. The formulations of interactive potential between $\mathrm{CO}_{2}$ molecules and the carbon wall are used to calculate the isosteric heat of adsorption as a function of pore widths. Employing adsorption isotherms and Qst models, a thermodynamic framework is developed to calculate the energetic performance or COP of adsorption cycle. The COP is presented here as a function of pore width. It is observed that there is no cooling performance for pore widths varying from 0 to $3 \AA$. The best performance is obtained for the pore widths ranging from 7 to 15 $\AA$ with higher limiting uptakes or pore volumes. The is found to be higher in micro-pore ranges. This indicates that the adsorption and desorption rates are faster in micro-pores. The carbonaceous materials should be designed with micro-pores ranging from 7 to $15 \AA$ and higher micro-pore volume for adsorption cooling applications. The knowledge of $q_{s t}{ }^{o}$ for an adsorbent - adsorbate system can help in the design of new carbonaceous materials such as carbon nanotubes, carbon fibres, pitch based activated carbon, metal organic frameworks, and carbonsilica composites. From the present analysis, we can approximately find the optimum ranges of pore widths that would be desirable to design porous activated carbons for the adsorption of carbon dioxide on carbon materials in refrigeration and cooling applications.

From the present analysis, it is noticed that silica gels optimised with respect to pore sizes and volumes for a given evaporating temperature can entail an increase of the COP and OPR of the order of 20 to $40 \%$, which cannot be neglected. If the evaporator temperature varies and especially when it decreases, there is a decreasing tendency of COP. Another conclusion of this study is that an increase of pore volume does not entail systematically an improvement of performance. The best performance of adsorption cooling cycle is obtained from a compromise between the greatest pore volume and the optimal pore size. From the present analysis, it is 
observed that there is no cooling and water production performance for pore widths varying from 0 to $0.3 \mathrm{~nm}$. The best performance of silica gel-water based cooling and desalination is obtained at the pore width of $0.9 \mathrm{~nm}$ with higher pore volume. The isosteric heat is found to be higher in micro-pore ranges. This indicates that the adsorption and desorption rates are higher in micro-

pores. The adsorbent materials like silica gel should be designed with micro-pores ranging from 0.8 to $1 \mathrm{~nm}$ and higher micro-pore volume for adsorption cooling and desalination applications.

\subsection{Future Work}

In the future, the following works can be carried out for the further improvement of modelling, simulation and performance optimisation of adsorption assisted cooling and desalination system.

1. To develop advanced molecular structure models for other types of adsorbent materials such as metal organic or other zeolite assisted frameworks that are more complicated than the lattice, amorphous surfaces and framework with channels.

2. To derive a pore model employing the real structure of porous material other than the simplified parallel slit-shaped and cylindrical pore models.

3. To investigate the phase transition and phase equilibrium for polar gas and solid surfaces with complicated molecular structures [154-159].

4. To design and fabricate novel adsorbent materials employing the proposed model and use them for the fabrication of adsorption chillers. 


\section{References}

[1] Dąbrowski, A., Adsorption - from theory to practice. Advances in Colloid and Interface Science, 2001. 93(1-3): p. 135-224.

[2] Wang, X.L.;Cai, W.J.;Lu, J.G.;Sun, Y.X.;Ding, X.D., A hybrid dehumidifier model for real-time performance monitoring, control and optimization in liquid desiccant dehumidification system. Applied Energy, 2013. 111: p. 449-455.

[3] Ng, K.C.;Thu, K.;Kim, Y.;Chakraborty, A.;Amy, G., Adsorption desalination: An emerging low-cost thermal desalination method. Desalination, 2013. 308: p. 161-179.

[4] Ng, K.C.;Thu, K.;Saha, B.B.;Chakraborty, A., Study on a waste heat-driven adsorption cooling cum desalination cycle. International Journal of Refrigeration-Revue Internationale Du Froid, 2012. 35(3): p. 685-693.

[5] Do, D.D., Adsorption Analysis : Equilibria and Kinetics. Series on chemical engineering. 1998, London: Imperial College Press. xxi, 892 p.

[6] Chakraborty, A.;Leong, K.C.;Thu, K.;Saha, B.B.;Ng, K.C., Theoretical insight of adsorption cooling. Applied Physics Letters, 2011. 98(22).

[7] Brunauer, S.;Deming, L.S.;Deming, W.E.;Teller, E., On a theory of the van der Waals adsorption of gases. Journal of the American Chemical Society, 1940. 62: p. 1723-1732.

[8] Donohue, M.D.;Aranovich, G.L., Classification of Gibbs adsorption isotherms. Advances in Colloid and Interface Science, 1998. 76-77: p. 137-152.

[9] Chakraborty, A.;Saha, B.B.;Koyama, S.;Ng, K.C., On the thermodynamic modeling of the isosteric heat of adsorption and comparison with experiments. Applied Physics Letters, 2006. 89(17). 
[10] Fan, W.;Chakraborty, A.;Kayal, S., Adsorption cooling cycles: Insights into carbon dioxide adsorption on activated carbons. Energy, 2016. 102: p. 491-501.

[11] Saha, B.B.;Chakraborty, A.;Koyama, S.;Aristov, Y.I., A new generation cooling device employing CaCl2-in-silica gel-water system. International Journal of Heat and Mass Transfer, 2009. 52(1-2): p. 516-524.

[12] Tian, Y.;Wu, J., Differential Heat of Adsorption and Isosteres. Langmuir, 2017. 33(4): p. 996-1003.

[13] Schindler, B.J.;Levan, M.D., The theoretical maximum isosteric heat of adsorption in the Henry's law region for slit-shaped carbon nanopores. Carbon, 2008. 46(4): p. 644-648.

[14] Fan, W.;Chakraborty, A., Investigation of the Interaction of Polar Molecules on Graphite Surface: Prediction of Isosteric Heat of Adsorption at Zero Surface Coverage. The Journal of Physical Chemistry C, 2016. 120(41): p. 23490-23499.

[15] Foo, K.Y.;Hameed, B.H., Insights into the modeling of adsorption isotherm systems. Chemical Engineering Journal, 2010. 156(1): p. 2-10.

[16] Ho, Y.S.;McKay, G., Kinetic Models for the Sorption of Dye from Aqueous Solution by Wood. Process Safety and Environmental Protection, 1998. 76(2): p. 183-191.

[17] Thu, K.;Kim, Y.D.;Myat, A.;Chakraborty, A.;Ng, K.C., Performance investigation of advanced adsorption desalination cycle with condenser-evaporator heat recovery scheme. Desalination and Water Treatment, 2013. 51(1-3): p. 150-163.

[18] Kayal, S.;Sun, B.C.;Saha, B.B., Adsorption characteristics of AQSOA zeolites and water for adsorption chillers. International Journal of Heat and Mass Transfer, 2016. 92: p. 11201127. 
[19] Sah, R.P.;Choudhury, B.;Das, R.K., A review on adsorption cooling systems with silica gel and carbon as adsorbents. Renewable and Sustainable Energy Reviews, 2015. 45: p. 123134.

[20] Reddy Panyam, V.;Banker, N.D., Thermodynamic assessment of a gas turbine power plant integrated with an adsorption refrigeration system. Applied Thermal Engineering, 2017. 117: p. 577-583.

[21] Hamdy, M.;Askalany, A.A.;Harby, K.;Kora, N., An overview on adsorption cooling systems powered by waste heat from internal combustion engine. Renewable and Sustainable Energy Reviews, 2015. 51: p. 1223-1234.

[22] Ali, S.M.;Chakraborty, A., Thermodynamic modelling and performance study of an engine waste heat driven adsorption cooling for automotive air-conditioning. Applied Thermal Engineering, 2015. 90: p. 54-63.

[23] Ali, E.S.;Harby, K.;Askalany, A.A.;Diab, M.R.;Alsaman, A.S., Weather effect on a solar powered hybrid adsorption desalination-cooling system: A case study of Egypt's climate. Applied Thermal Engineering, 2017. 124: p. 663-672.

[24] Alsaman, A.S.;Askalany, A.A.;Harby, K.;Ahmed, M.S., Performance evaluation of a solar-driven adsorption desalination-cooling system. Energy, 2017. 128: p. 196-207.

[25] Zheng, H., Absorption and Adsorption Solar Desalination System. 2017: p. 623-670.

[26] Alsaman, A.S.;Askalany, A.A.;Harby, K.;Ahmed, M.S., A state of the art of hybrid adsorption desalination-cooling systems. Renewable and Sustainable Energy Reviews, 2016. 58: p. 692-703.

[27] Brunauer, S.;Emmett, P.H.;Teller, E., Adsorption of Gases in Multimolecular Layers. Journal of the American Chemical Society, 1938. 60(2): p. 309-319. 
[28] Brunauer, S., The Adsorption of Gases and Vapours. 1943, London: Geoffrey Cumberlege. vii, $511 \mathrm{p}$.

[29] McMillan, W.G.;Teller, E., The Assumptions of the B.E.T. Theory. The Journal of Physical Chemistry, 1951. 55(1): p. 17-20.

[30] Frenkel, J., Kinetic theory of liquids. 1946, Oxford: Clarendon Press. xi, 488 p.

[31] Halsey, G., Physical Adsorption on Non - Uniform Surfaces. The Journal of Chemical Physics, 1948. 16(10): p. 931-937.

[32] Hill, T.L., Theory of Physical Adsorption, in Advances in Catalysis, W.G. Frankenburg, V.I.K.;Rideal, E.K., Editors. 1952, Academic Press. p. 211-258.

[33] Chakraborty, A.;Sun, B.C., An adsorption isotherm equation for multi-types adsorption with thermodynamic correctness. Applied Thermal Engineering, 2014. 72(2): p. 190-199.

[34] Sun, B.;Chakraborty, A., Thermodynamic formalism of water uptakes on solid porous adsorbents for adsorption cooling applications. Applied Physics Letters, 2014. 104(20).

[35] Toth, J., Adsorption: Theory, Modeling and Analysis. Surfice Science. Vol. 107. 2002, New York: Marcel Dekker Inc.

[36] Hamdaoui, O.;Naffrechoux, E., Modeling of adsorption isotherms of phenol and chlorophenols onto granular activated carbon - Part II. Models with more than two parameters. Journal of Hazardous Materials, 2007. 147(1-2): p. 401-411.

[37] Goldberg, S., Equations and Models Describing Adsorption Processes in Soils. Chemical Processes in Soils, 2005(chemicalprocess): p. 489-517.

[38] Lagergren, S., About the theory of so-called adsorption of soluble substances. Kungliga Svenska Vetenskapsakademiens. Handlingar, 1898 24(4): p. 1-39. 
[39] Ho, Y.S.;McKay, G., Pseudo-second order model for sorption processes. Process Biochemistry, 1999. 34(5): p. 451-465.

[40] Wu, F.C.;Tseng, R.L.;Juang, R.S., Kinetic modeling of liquid-phase adsorption of reactive dyes and metal ions on chitosan. Water Research, 2001. 35(3): p. 613-618.

[41] Ho, Y.S.;McKay, G., Comparative sorption kinetic studies of dye and aromatic compounds onto fly ash. Journal of Environmental Science and Health, Part A, 1999. 34(5): p. 11791204.

[42] Lakhlifi, A.;Killingbeck, J.P., Investigation of the interaction of some astrobiological molecules with the surface of a graphite (0001) substrate. Application to the CO, HCN, H2O and H2CO molecules. Surface Science, 2010. 604(1): p. 38-46.

[43] Klomkliang, N.;Kaewmanee, R.;Saimoey, S.;Intarayothya, S.;Do, D.D.;Nicholson, D., Adsorption of water and methanol on highly graphitized thermal carbon black: The effects of functional group and temperature on the isosteric heat at low loadings. Carbon, 2016. 99: p. 361-369.

[44] Birkett, G.R.;Do, D.D., Simulation study of methanol and ethanol adsorption on graphitized carbon black. Molecular Simulation, 2006. 32(10-11): p. 887-899.

[45] Chakraborty, A.;Thu, K.;Ng, K.C., Advanced Adsorption Cooling cum Desalination Cycle: A Thermodynamic Framework. 2011: p. 605-610.

[46] Pons, M.;Meunier, F.;Cacciola, G.;Critoph, R.E.;Groll, M.;Puigjaner, L.;Spinner, B.;Ziegler, F., Thermodynamic based comparison of sorption systems for cooling and heat pumping. International Journal of Refrigeration, 1999. 22(1): p. 5-17.

[47] Chakraborty, A.;Thu, K.;Saha, B.B.;Ng, K.C., Adsorption-Desalination Cycle, in Advances in Water Desalination. 2012, John Wiley \& Sons, Inc. p. 377-451. 
[48] Lu, Z.S.;Wang, R.Z.;Xia, Z.Z.;Gong, L.X., Experimental investigation adsorption chillers using micro-porous silica gel-water and compound adsorbent-methanol. Energy Conversion and Management, 2013. 65: p. 430-437.

[49] Liu, Y.;Leong, K.C., Numerical study of a novel cascading adsorption cycle. International Journal of Refrigeration-Revue Internationale Du Froid, 2006. 29(2): p. 250-259.

[50] Thu, K.;Chakraborty, A.;Kim, Y.D.;Myat, A.;Saha, B.B.;Ng, K.C., Numerical simulation and performance investigation of an advanced adsorption desalination cycle. Desalination, 2013. 308: p. 209-218.

[51] Jribi, S.;Koyama, S.;Saha, B.B., Performance Investigation of a Novel CO2 CompressionAdsorption Based Hybrid Cooling Cycle. . Engineering Sciences Reports, Kyushu University, 2010. 32(3): p. 12-18.

[52] Askalany, A.A.;Saha, B.B.;Kariya, K.;Ismail, I.M.;Salem, M.;Ali, A.H.H.;Morsy, M.G., Hybrid adsorption cooling systems-An overview. Renewable \& Sustainable Energy Reviews, 2012. 16(8): p. 5787-5801.

[53] Habib, K.;Saha, B.B.;Chakraborty, A.;Oh, S.T.;Koyama, S., Study on solar driven combined adsorption refrigeration cycles in tropical climate. Applied Thermal Engineering, 2013. 50(2): p. 1582-1589.

[54] Habib, K.;Saha, B.B.;Chakraborty, A.;Koyama, S.;Srinivasan, K., Performance evaluation of combined adsorption refrigeration cycles. International Journal of Refrigeration-Revue Internationale Du Froid, 2011. 34(1): p. 129-137.

[55] Lu, Z.S.;Wang, R.Z., Performance improvement and comparison of mass recovery in CaCl2/activated carbon adsorption refrigerator and silica gel/LiCl adsorption chiller 
driven by low grade waste heat. International Journal of Refrigeration-Revue Internationale Du Froid, 2013. 36(5): p. 1504-1511.

[56] Wang, X.L.;Chua, H.T., Two bed silica gel-water adsorption chillers: An effectual lumped parameter model. International Journal of Refrigeration-Revue Internationale Du Froid, 2007. 30(8): p. 1417-1426.

[57] Chakraborty, A.;Saha, B.B.;Aristov, Y.I., Dynamic behaviors of adsorption chiller: Effects of the silica gel grain size and layers. Energy, 2014. 78: p. 304-312.

[58] Aristov, Y.I., Optimal adsorbent for adsorptive heat transformers: Dynamic considerations. International Journal of Refrigeration-Revue Internationale Du Froid, 2009. 32(4): p. 675-686.

[59] Miyazaki, T.;Akisawa, A.;Saha, B.B.;El-Sharkawy, I.I.;Chakraborty, A., A new cycle time allocation for enhancing the performance of two-bed adsorption chillers. International Journal of Refrigeration-Revue Internationale Du Froid, 2009. 32(5): p. 846-853.

[60] Li, A.;Bin Ismail, A.;Thu, K.;Ng, K.C.;Loh, W.S., Performance evaluation of a zeolitewater adsorption chiller with entropy analysis of thermodynamic insight. Applied Energy, 2014. 130: p. 702-711.

[61] Qian, S.X.;Gluesenkamp, K.;Hwang, Y.;Radermacher, R.;Chun, H.H., Cyclic steady state performance of adsorption chiller with low regeneration temperature zeolite. Energy, 2013. 60: p. $517-526$.

[62] Restuccia, G.;Freni, A.;Russo, F.;Vasta, S., Experimental investigation of a solid adsorption chiller based on a heat exchanger coated with hydrophobic zeolite. Applied Thermal Engineering, 2005. 25(10): p. 1419-1428. 
[63] Gong, L.X.;Wang, R.Z.;Xia, Z.Z.;Lu, Z.S., Experimental study on an adsorption chiller employing lithium chloride in silica gel and methanol. International Journal of Refrigeration-Revue Internationale Du Froid, 2012. 35(7): p. 1950-1957.

[64] Saha, B.B.;EI-Sharkawy, I.I.;Chakraborty, A.;Koyama, S., Study on an activated carbon fiber-ethanol adsorption chiller: Part I - system description and modelling. International Journal of Refrigeration-Revue Internationale Du Froid, 2007. 30(1): p. 86-95.

[65] Jribi, S.;Saha, B.B.;Koyama, S.;Bentaher, H., Modeling and simulation of an activated carbon-CO2 four bed based adsorption cooling system. Energy Conversion and Management, 2014. 78: p. 985-991.

[66] Tso, C.Y.;Chao, C.Y.H.;Fu, S.C., Performance analysis of a waste heat driven activated carbon based composite adsorbent - Water adsorption chiller using simulation model. International Journal of Heat and Mass Transfer, 2012. 55(25-26): p. 7596-7610.

[67] Tso, C.Y.;Chao, C.Y.H., Activated carbon, silica-gel and calcium chloride composite adsorbents for energy efficient solar adsorption cooling and dehumidification systems. International Journal of Refrigeration-Revue Internationale Du Froid, 2012. 35(6): p. 16261638.

[68] Wu, J.W.;Biggs, M.J.;Hu, E.J., Dynamic model for the optimisation of adsorption-based desalination processes. Applied Thermal Engineering, 2014. 66(1-2): p. 464-473.

[69] Ng, K.C.;Chua, H.T.;Chung, C.Y.;Loke, C.H.;Kashiwagi, T.;Akisawa, A.;Saha, B.B., Experimental investigation of the silica gel-water adsorption isotherm characteristics. Applied Thermal Engineering, 2001. 21(16): p. 1631-1642.

[70] Mitra, S.;Kumar, P.;Srinivasan, K.;Dutta, P., Simulation study of a two-stage adsorber system. Applied Thermal Engineering, 2014. 72(2): p. 283-288. 
[71] Thu, K.;Saha, B.B.;Chakraborty, A.;Chun, W.G.;Ng, K.C., Study on an advanced adsorption desalination cycle with evaporator-condenser heat recovery circuit. International Journal of Heat and Mass Transfer, 2011. 54(1-3): p. 43-51.

[72] Thu, K.;Ng, K.C.;Saha, B.B.;Chakraborty, A.;Koyama, S., Operational strategy of adsorption desalination systems. International Journal of Heat and Mass Transfer, 2009. 52(7-8): p. 1811-1816.

[73] Ng, K.C.;Thu, K.;Oh, S.J.;Ang, L.;Shahzad, M.W.;Bin Ismail, A., Recent developments in thermally-driven seawater desalination: Energy efficiency improvement by hybridization of the MED and AD cycles. Desalination, 2015. 356: p. 255-270.

[74] Shahzad, M.W.;Ng, K.C.;Thu, K.;Saha, B.B.;Chun, W.G., Multi effect desalination and adsorption desalination (MEDAD): A hybrid desalination method. Applied Thermal Engineering, 2014. 72(2): p. 289-297.

[75] Thu, K.;Kim, Y.D.;Amy, G.;Chun, W.G.;Ng, K.C., A hybrid multi-effect distillation and adsorption cycle. Applied Energy, 2013. 104: p. 810-821.

[76] Kim, Y.-D.;Thu, K.;Masry, M.E.;Ng, K.C., Water quality assessment of solar-assisted adsorption desalination cycle. Desalination, 2014. 344: p. 144-151.

[77] Xu, S.Z.;Wang, L.W.;Wang, R.Z., Thermodynamic analysis of single-stage and multistage adsorption refrigeration cycles with activated carbon-ammonia working pair. Energy Conversion and Management, 2016. 117: p. 31-42.

[78] Wang, L.W.;Metcalf, S.J.;Critoph, R.E.;Thorpe, R.;Tamainot-Telto, Z., Development of thermal conductive consolidated activated carbon for adsorption refrigeration. Carbon, 2012. 50(3): p. 977-986. 
[79] Ghazy, M.;Askalany, A.A.;Harby, K.;Ahmed, M.S., Adsorption isotherms and kinetics of HFC-404A onto bituminous based granular activated carbon for storage and cooling applications. Applied Thermal Engineering, 2016. 105: p. 639-645.

[80] Werder, T.;Walther, J.H.;Jaffe, R.L.;Halicioglu, T.;Koumoutsakos, P., On the watercarbon interaction for use in molecular dynamics simulations of graphite and carbon nanotubes. Journal of Physical Chemistry B, 2003. 107(6): p. 1345-1352.

[81] Carlos, W.E.;Cole, M.W., Interaction between a He atom and a graphite surface. Surface Science, 1980. 91(1): p. 339-357.

[82] Wang, M.X.;Huang, Z.H.;Lv, W.;Yang, Q.H.;Kang, F.Y.;Liang, K.M., Water vapor adsorption on low-temperature exfoliated graphene nanosheets. Journal of Physics and Chemistry of Solids, 2012. 73(12): p. 1440-1443.

[83] von Alfthan, S.;Kuronen, A.;Kaski, K., Realistic models of amorphous silica: A comparative study of different potentials. Physical Review B, 2003. 68(7): p. 073203.

[84] Kierys, A.;Goworek, J.;Rawski, M.;Halasz, I., On the molecular basis of silica gel morphology. Advanced Materials Letters, 2015. 6(1): p. 40-46.

[85] Che, M.;Mori, K.;Yamashita, H., Elaboration, characterization and properties of silicabased single-site heterogeneous photocatalysts. Proceedings of the Royal Society aMathematical Physical and Engineering Sciences, 2012. 468(2143): p. 2113-2128.

[86] Hench, L.L.;West, J.K., Molecular-Orbital Models of Silica. Annual Review of Materials Science, 1995. 25: p. 37-68.

[87] Bnown, G.E.;Grnns, G.V., Oxygen Coordination and the Si-O Bond. American Mineralogist, 1969. 54: p. 1528-1539. 
[88] Raymonda, J.W.;Muenter, J.S.;Klempere.Wa, Electric Dipole Moment of Sio and Geo. Journal of Chemical Physics, 1970. 52(7): p. 3458-\&.

[89] Liu, J.;Yu, J., Chapter 1 - Toward Greener and Designed Synthesis of Zeolite Materials A2 - Sels, Bert F, in Zeolites and Zeolite-Like Materials, Kustov, L.M., Editor. 2016, Elsevier: Amsterdam. p. 1-32.

[90] Ilić, B.;Wettstein, S.G., A review of adsorbate and temperature-induced zeolite framework flexibility. Microporous and Mesoporous Materials, 2017. 239: p. 221-234.

[91] Liu, Z.;Wakihara, T.;Nomura, N.;Matsuo, T.;Anand, C.;Elangovan, S.P.;Yanaba, Y.;Yoshikawa, T.;Okubo, T., Ultrafast and Continuous Flow Synthesis of Silicoaluminophosphates. Chemistry of Materials, 2016. 28(13): p. 4840-4847.

[92] Flanigen, E.M.;Lok, B.M.;Patton, R.L.;Wilson, S.T., Aluminophosphate Molecular-Sieves and the Periodic-Table. Pure and Applied Chemistry, 1986. 58(10): p. 1351-1358.

[93] Bojan, M.J.;Steele, W.A., Chapter Four - Monte Carlo and Molecular Dynamics, in Adsorption by Carbons. 2008, Elsevier: Amsterdam. p. 77-101.

[94] Horikawa, T.;Zeng, Y.;Do, D.D.;Sotowa, K.;Alcantara Avila, J.R., On the isosteric heat of adsorption of non-polar and polar fluids on highly graphitized carbon black. J Colloid Interface Sci, 2015. 439: p. 1-6.

[95] Zachariasen, W.H., The atomic arrangement in glass. Journal of the American Chemical Society, 1932. 54: p. 3841-3851.

[96] Zhuravlev, L.T., The surface chemistry of amorphous silica. Zhuravlev model. Colloids and Surfaces a-Physicochemical and Engineering Aspects, 2000. 173(1-3): p. 1-38.

[97] Wooten, F.;Winer, K.;Weaire, D., Computer-Generation of Structural Models of Amorphous Si and Ge. Physical Review Letters, 1985. 54(13): p. 1392-1395. 
[98] Barkema, G.T.;Mousseau, N., High-quality continuous random networks. Physical Review B, 2000. 62(8): p. 4985-4990.

[99] McGreevy, R.L., Reverse Monte Carlo modelling. Journal of Physics-Condensed Matter, 2001. 13(46): p. R877-R913.

[100] Muller, C.R.;Kathriarachchi, V.;Schuch, M.;Maass, P.;Petkov, V.G., Reverse Monte Carlo modeling of ion conducting network glasses: An evaluation based on molecular dynamics simulations. Physical Chemistry Chemical Physics, 2010. 12(35): p. 10444-10451.

[101] Biswas, P.;Atta-Fynn, R.;Drabold, D.A., Reverse Monte Carlo modeling of amorphous silicon. Physical Review B, 2004. 69(19).

[102] Peng, L.;Qisui, W.;Xi, L.;Chaocan, Z., Investigation of the states of water and OH groups on the surface of silica. Colloids and Surfaces a-Physicochemical and Engineering Aspects, 2009. 334(1-3): p. 112-115.

[103] Leed, E.A.;Pantano, C.G., Computer modeling of water adsorption on silica and silicate glass fracture surfaces. Journal of Non-Crystalline Solids, 2003. 325(1-3): p. 48-60.

[104] Liu, J.;LeVan, M.D., Henry's law constants and isosteric heats of adsorption at zero loading for multi-wall carbon surfaces with different geometries. Carbon, 2010. 48(12): p. 3454-3462.

[105] Steele, W.A., The interaction of gases with solid surfaces. 1st ed. The International encyclopedia of physical chemistry and chemical physics Topic 14: Properties of interfaces,. 1974, Oxford ; New York: Pergamon Press. xi, 349 p.

[106] Ionov, S.I.;LaVilla, M.E., Probing the molecule-surface interaction via inversion symmetry changes in the scattering of state - selected ND3 on graphite (0001). The Journal of Chemical Physics, 1992. 97(12): p. 9379-9388. 
[107] Buckingham, A.D., Permanent and Induced Molecular Moments and Long-Range Intermolecular Forces. Advances in Chemical Physics, 1967. 12: p. 107-142.

[108] Jorgensen, W.L.;Chandrasekhar, J.;Madura, J.D.;Impey, R.W.;Klein, M.L., Comparison of Simple Potential Functions for Simulating Liquid Water. Journal of Chemical Physics, 1983. 79(2): p. 926-935.

[109] Petkov, V.;Billinge, S.J.L.;Shastri, S.D.;Himmel, B., Polyhedral units and network connectivity in calcium aluminosilicate glasses from high-energy X-ray diffraction. Physical Review Letters, 2000. 85(16): p. 3436-3439.

[110] Le Roux, S.;Petkov, V., ISAACS - interactive structure analysis of amorphous and crystalline systems. Journal of Applied Crystallography, 2010. 43: p. 181-185.

[111] Hoang, V.V., Molecular dynamics simulation of amorphous SiO2 nanoparticles. J Phys Chem B, 2007. 111(44): p. 12649-56.

[112] Kohara, S.;Suzuya, K., Intermediate-range order in vitreous SiO2 and GeO2. Journal of Physics-Condensed Matter, 2005. 17(5): p. S77-S86.

[113] Cruz-Chu, E.R.;Aksimentiev, A.;Schulten, K., Water-silica force field for simulating nanodevices. J Phys Chem B, 2006. 110(43): p. 21497-508.

[114] Lakhlifi, A.;Killingbeck, J.P., Dynamic and spectroscopic studies of single molecules physisorbed on graphite substrates. 2. Application to the ammonia molecule. Journal of Physical Chemistry B, 2005. 109(22): p. 11322-11331.

[115] Kakiuchi, H.;Shimooka, S.;Iwade, M.;Oshima, K.;Yamazaki, M.;Terada, S.;Watanabe, H.;Takewaki, T., Novel Water Vapor Adsorbent FAM-ZO1 and its Applicability to an Adsorption Heat Pump. KAGAKU KOGAKU RONBUNSHU, 2005. 31(5): p. 361-364. 
[116] Shimooka, S.;Oshima, K.;Hidaka, H.;Takewaki, T.;Kakiuchi, H.;Kodama, A.;Kubota, M.;Matsuda, H., The Evaluation of Direct Cooling and Heating Desiccant Device Coated with FAM. JOURNAL OF CHEMICAL ENGINEERING OF JAPAN, 2007. 40(13): p. 1330-1334.

[117] Kakiuchi, H.;Shimooka, S.;Iwade, M.;Oshima, K.;Yamazaki, M.;Terada, S.;Watanabe, H.;Takewaki, T., Water Vapor Adsorbent FAM-Z02 and Its Applicability to Adsorption Heat Pump. KAGAKU KOGAKU RONBUNSHU, 2005. 31(4): p. 273-277.

[118] Everett, D.H.;Powl, J.C., Adsorption in slit-like and cylindrical micropores in the henry's law region. A model for the microporosity of carbons. Journal of the Chemical Society, Faraday Transactions 1: Physical Chemistry in Condensed Phases, 1976. 72(0): p. 619-636.

[119] Savitz, S.;Siperstein, F.;Gorte, R.J.;Myers, A.L., Calorimetric Study of Adsorption of Alkanes in High-Silica Zeolites. The Journal of Physical Chemistry B, 1998. 102(35): p. $6865-6872$.

[120] Lobo, R.F., Chapter 9 - Intermolecular Forces in Zeolite Adsorption and Catalysis, in Ordered Porous Solids. 2009, Elsevier: Amsterdam. p. 239-261.

[121] Rappe, A.K.;Casewit, C.J.;Colwell, K.S.;Goddard, W.A.;Skiff, W.M., Uff, a Full PeriodicTable Force-Field for Molecular Mechanics and Molecular-Dynamics Simulations. Journal of the American Chemical Society, 1992. 114(25): p. 10024-10035.

[122] Faro, T.M.C.;Thim, G.P.;Skaf, M.S., A Lennard-Jones plus Coulomb potential for Al3+ ions in aqueous solutions. The Journal of Chemical Physics, 2010. 132(11): p. 114509.

[123] Jiang, J.-W.;Park, H.S., A Gaussian treatment for the friction issue of Lennard-Jones potential in layered materials: Application to friction between graphene, MoS2, and black phosphorus. Journal of Applied Physics, 2015. 117(12): p. 124304. 
[124] Kusalik, P.G.;Svishchev, I.M., The Spatial Structure in Liquid Water. Science, 1994. 265(5176): p. 1219-1221.

[125] Steele, W.A., Physical Interaction of Gases with Crystalline Solids .1. Gas-Solid Energies and Properties of Isolated Adsorbed Atoms. Surface Science, 1973. 36(1): p. 317-352.

[126] Abascal, J.L.F.;Vega, C., The water forcefield: Importance of dipolar and quadrupolar interactions. Journal of Physical Chemistry C, 2007. 111(43): p. 15811-15822.

[127] Hansen, F.Y.;Bruch, L.W.;Roosevelt, S.E., Electrostatic Forces and the FrequencySpectrum of a Monolayer Solid of Linear-Molecules on Graphite. Physical Review B, 1992. 45(19): p. 11238-11248.

[128] Vidali, G.;Cole, M.W., Lateral Variation of the Physisorption Potential for Noble-Gases on Graphite. Physical Review B, 1984. 29(12): p. 6736-6738.

[129] Spassova, M.;Monev, V.;Kanev, I.;Champagne, B.;Mosley, D.H.;J.M., A., Ab Initio Summation Over States/SCI for Static and Dynamic Hyperpolarizabilities of Small Molecules. Quantum Systems in Chemistry and Physics, ed. al, H.-L.e. Vol. Basic Problems and Model Systems. 2000, Great Britain: Kluwer Academic Publishers.

[130] Chelli, R.;Pagliai, M.;Procacci, P.;Cardini, G.;Schettino, V., Polarization response of water and methanol investigated by a polarizable force field and density functional theory calculations: Implications for charge transfer. Journal of Chemical Physics, 2005. 122(7).

[131] DiStasio, R.A.;Gobre, V.V.;Tkatchenko, A., Many-body van der Waals interactions in molecules and condensed matter. Journal of Physics: Condensed Matter, 2014. 26(21).

[132] Avgul, N.N.;Kieslev, A.V., Chemistry and Physics of Carbon, ed. Walker, P.L. 1970, New York: Marcel Dekker. 
[133] Markovic, N.;Andersson, P.U.;Nagard, M.B.;Pettersson, J.B.C., Scattering of water from graphite: simulations and experiments. Chemical Physics, 1999. 247(3): p. 413-430.

[134] Lin, C.S.;Zhang, R.Q.;Lee, S.T.;Elstner, M.;Frauenheim, T.;Wan, L.J., Simulation of water cluster assembly on a graphite surface. Journal of Physical Chemistry B, 2005. 109(29): p. $14183-14188$.

[135] Rubeš, M.;Nachtigall, P.;Vondrášek, J.;Bludský, O., Structure and Stability of the Water-Graphite Complexes. The Journal of Physical Chemistry C, 2009. 113(19): p. 8412-8419.

[136] Feller, D.;Jordan, K.D., Estimating the strength of the water/single-layer graphite interaction. Journal of Physical Chemistry A, 2000. 104(44): p. 9971-9975.

[137] Pertsin, A.;Grunze, M., Water-graphite interaction and behavior of water near the graphite surface. Journal of Physical Chemistry B, 2004. 108(4): p. 1357-1364.

[138] Liu, J.C.;Monson, P.A., Monte Carlo simulation study of water adsorption in activated carbon. Industrial and Engineering Chemistry Research, 2006. 45(16): p. 5649-5656.

[139] Karapetian, K.;Jordan, K.D., Water in Confined Environments, ed. Devlin, J.P.;Buch, V. 2003, New York: Springer.

[140] Sanfelix, P.C.;Holloway, S.;Kolasinski, K.W.;Darling, G.R., The structure of water on the (0001) surface of graphite. Surface Science, 2003. 532: p. 166-172.

[141] Karlicky, F.;Otyepkova, E.;Banas, P.;Lazar, P.;Kocman, M.;Otyepka, M., Interplay between Ethanol Adsorption to High-Energy Sites and Clustering on Graphene and Graphite Alters the Measured Isosteric Adsorption Enthalpies. Journal of Physical Chemistry C, 2015. 119(35): p. 20535-20543. 
[142] Chua, H.T.;Ng, K.C.;Chakraborty, A.;Oo, N.M.;Othman, M.A., Adsorption characteristics of silica gel plus water systems. Journal of Chemical and Engineering Data, 2002. 47(5): p. $1177-1181$.

[143] Chakraborty, A.;Saha, B.B.;Koyama, S.;Ng, K.C.;Srinivasan, K., Adsorption Thermodynamics of Silica Gel-Water Systems. Journal of Chemical and Engineering Data, 2009. 54(2): p. 448-452.

[144]Lemmon, E.W.;Huber, M.L.;McLinden.M.O., NIST Standard Reference Database 23: Reference Fluid Thermodynamic and Transport Properties-REFPROP, Version 8.0, Program, S.R.D., Editor. 2007, National Institute of Standards and Technology: Gaithersburg.

[145] Tóth, J., Thermodynamical Correctness of Gas/Solid Adsorption Isotherm Equations. Journal of Colloid and Interface Science, 1994. 163(2): p. 299-302.

[146] McEnaney, B., Estimation of the dimensions of micropores in active carbons using the Dubinin-Radushkevich equation. Carbon, 1987. 25(1): p. 69-75.

[147] NACC, PTX data for silica gel/water pair, Manufacturer's Proprietary Data. Nishiyodo Air Conditioning Co Ltd.Tokyo, Japan, 1992.

[148] Khalfaoui, M.;Knani, S.;Hachicha, M.A.;Lamine, A.B., New theoretical expressions for the five adsorption type isotherms classified by BET based on statistical physics treatment. Journal of Colloid and Interface Science, 2003. 263(2): p. 350-356.

[149] Chakraborty, A.;Saha, B.B.;Ng, K.C.;Koyama, S.;Srinivasan, K., Theoretical insight of physical adsorption for a single-component adsorbent+adsorbate system: I. Thermodynamic property surfaces. Langmuir, 2009. 25(4): p. 2204-11. 
[150] Critoph, R.E.;Metcalf, S.J., Specific cooling power intensification limits in ammoniacarbon adsorption refrigeration systems. Applied Thermal Engineering, 2004. 24(5-6): p. 661-678.

[151] Ravikovitch, P.I.;Vishnyakov, A.;Neimark, A.V., Density functional theories and molecular simulations of adsorption and phase transitions in nanopores. Physical Review E, 2001. 64(1).

[152] Ribeiro, R.P.;Sauer, T.P.;Lopes, F.V.;Moreira, R.F.;Grande, C.A.;Rodrigues, A.r.E., Adsorption of CO2, CH4, and N2in Activated Carbon Honeycomb Monolith. Journal of Chemical \& Engineering Data, 2008. 53(10): p. 2311-2317.

[153] Mclean, A.D.;Yoshimine, M., Higher Polarizabilities of Linear Molecules. Journal of Chemical Physics, 1967. 46(9): p. 3682-+.

[154] Norman, G.E.;Filinov, V.S., Investigations of Phase Transitions by a Monte-Carlo Method. High Temperature, 1969. 7(2): p. 216-\&.

[155] Amar, J.G., The Monte Carlo method in science and engineering. Computing in Science \& Engineering, 2006. 8(2): p. 9-19.

[156] Smykowski, D.;Szyja, B.;Szczygieł, J., GCMC simulations of CO2 adsorption on zeolitesupported Ir4 clusters. Journal of Molecular Graphics and Modelling, 2014. 50: p. 35-43.

[157] Cheng, J.R.;Yuan, X.H.;Zhao, L.;Huang, D.C.;Zhao, M.;Dai， L.;Ding, R., GCMC simulation of hydrogen physisorption on carbon nanotubes and nanotube arrays. Carbon, 2004. 42(10): p. 2019-2024.

[158] Mahdizadeh, S.J.;Goharshadi, E.K., Hydrogen storage on silicon, carbon, and silicon carbide nanotubes: A combined quantum mechanics and grand canonical Monte Carlo simulation study. International Journal of Hydrogen Energy, 2014. 39(4): p. 1719-1731. 
[159] Wu, Y.;Chen, H.Y.;Liu, D.F.;Qian, Y.;Xi, H.X., Adsorption and separation of ethanelethylene on ZIFs with various topologies: Combining GCMC simulation with the ideal adsorbed solution theory (IAST). Chemical Engineering Science, 2015. 124: p. 144153. 


\section{Appendices}

\section{A. Computational Strategy}

\section{A1. Multipole Expansion of Energy}

The energy of uncharged molecule in static electric filed can be expressed by multipole expansion formulation $[1,2]$,

$$
\begin{aligned}
\mathrm{U}=\mathrm{U}^{0}-\boldsymbol{\mu}_{\alpha} \boldsymbol{F}_{\alpha} & -\frac{1}{3} \boldsymbol{\Theta}_{\alpha \beta} \boldsymbol{F}_{\alpha \beta}-\frac{1}{15} \boldsymbol{\Omega}_{\alpha \beta \gamma} \boldsymbol{F}_{\alpha \beta \gamma}-\frac{1}{105} \boldsymbol{\Phi}_{\alpha \beta \gamma \delta} \boldsymbol{F}_{\alpha \beta \gamma \delta}+\cdots \\
& -\frac{1}{2} \boldsymbol{\alpha}_{\alpha \beta} \boldsymbol{F}_{\alpha} \boldsymbol{F}_{\beta}-\frac{1}{3} \boldsymbol{A}_{\alpha, \beta \gamma} \boldsymbol{F}_{\alpha} \boldsymbol{F}_{\beta \gamma \delta}-\frac{1}{6} \boldsymbol{C}_{\alpha \beta, \gamma \delta} \boldsymbol{F}_{\alpha \beta} \boldsymbol{F}_{\gamma \delta}-\frac{1}{15} \boldsymbol{E}_{\alpha, \beta \gamma \delta} \boldsymbol{F}_{\alpha} \boldsymbol{F}_{\beta \gamma \delta \delta}+\cdots \\
& -\frac{1}{6} \boldsymbol{\beta}_{\alpha \beta \gamma} \boldsymbol{F}_{\alpha} \boldsymbol{F}_{\beta} \boldsymbol{F}_{\gamma}-\frac{1}{6} \boldsymbol{B}_{\alpha \beta, \gamma \delta} \boldsymbol{F}_{\alpha} \boldsymbol{F}_{\beta} \boldsymbol{F}_{\gamma \delta}+\cdots \\
& -\frac{1}{24} \boldsymbol{\gamma}_{\alpha \beta \gamma \delta} \boldsymbol{F}_{\alpha} \boldsymbol{F}_{\beta} \boldsymbol{F}_{\gamma} \boldsymbol{F}_{\delta}+\cdots
\end{aligned}
$$

where $\mathrm{U}^{0}$ is the energy of free molecule, $\boldsymbol{\mu}_{\alpha}, \boldsymbol{\Theta}_{\alpha \beta}, \boldsymbol{\Omega}_{\alpha \beta \gamma}$ and $\boldsymbol{\Phi}_{\alpha \beta \gamma \delta}$ are dipole, quadrupole, octupole and hexadecapole moment, $\boldsymbol{\alpha}_{\alpha \beta}, \boldsymbol{\beta}_{\alpha \beta \gamma}$ and $\boldsymbol{\gamma}_{\alpha \beta \gamma \delta}$ are dipole polarizabilities, $\boldsymbol{A}_{\alpha, \beta \gamma}$, $\boldsymbol{C}_{\alpha \beta, \gamma \delta}, \boldsymbol{E}_{\alpha, \beta \gamma \delta}$ and $\boldsymbol{B}_{\alpha \beta, \gamma \delta}$ are the quadrupole polarizabilities, $\boldsymbol{F}$ with subscripts are electric field, electric field gradient (EFG), etc. depends on the number of groups of subscripts. By applying the specific reduction strategies on equation (4.5) based on the multipole properties of the molecule [3], the multipole expression is suitable for the calculation of interaction energy of molecules.

\section{A2. Reduced Multipole Expansion of Energy}

Taking Lennard-Jones (LJ) and quadrupole-quadrupole interaction into consideration, Pople [4] modified the potential and is given by,

$$
\mathrm{U}=\mathrm{u}_{\mathrm{LJ}}+u_{\text {quadrupole-quadrupole }}
$$


Considering Keesom Force between dipolar molecules, Keesom [5] also proposed the improved intermolecular potential model as

$$
\mathrm{U}=\mathrm{U}_{\mathrm{HS}}+\mathrm{U}_{\text {dipole-dipole }} \text { or } \mathrm{U}=\mathrm{U}_{\mathrm{HS}}+\mathrm{U}_{\text {quadrupole-quadrupole }}
$$

For non-polar molecules, the interaction potential can be expressed by LJ potential sufficiently while electrostatic and induction effect cannot be neglected for polar molecules. Therefore, by combining the Lenard-Jones, electrostatic and induction potential $\mathrm{U}_{\mathrm{LJ}}, \mathrm{U}_{\mathrm{E}}, \mathrm{U}_{\mathrm{I}}$, the total interaction potential $U_{m M}$ is proposed to obtain more complete description of interaction potential for polar molecules.

$$
\mathrm{U}_{\mathrm{mM}}=\mathrm{U}_{\mathrm{LJ}}+\mathrm{U}_{\mathrm{E}}+\mathrm{U}_{\mathrm{I}}
$$

\section{A3. Electric Field and Energy of multipole}

\section{Potential of dipole in static electric field}

The potential of permanent dipole in electric field $\mathbf{F}$ is,

$$
\mathrm{U}=-\boldsymbol{\mu} \cdot \mathbf{F}
$$

A permanent dipole placed in static electric field will induce a temporary dipole with the same dipole moment and opposite direction. In the non-liner regime, the induced dipole moment $\boldsymbol{\mu}^{\mathrm{I}}$ is,

$$
\boldsymbol{\mu}^{\mathrm{I}}=\boldsymbol{\alpha} \cdot \mathbf{F},
$$

where $\boldsymbol{\alpha}$ is the dipole moment polarizability tensor of an acceptor of electric field and $\mathbf{F}$ is the exerted static electric field. Due to the induced dipole instead of a permanent one, the induction potential $\mathrm{U}_{\mathrm{I}}$ is,

$$
\mathrm{U}_{\mathrm{I}}=-\frac{1}{2} \boldsymbol{\mu}^{\mathrm{I}} \cdot \mathbf{F}
$$

The Debye Force between dipole and induced dipole adds to the intermolecular potential when non-zero dipole moment exists in the interaction pair. Taking this Induction Effect into account, the interaction potential $U_{I}$ is, 


$$
\mathrm{U}=\mathrm{U}_{\mathrm{I}}=-\frac{1}{2} \boldsymbol{\alpha} \cdot \mathbf{F}^{2}
$$

\section{Electric field generated by a dipole}

Generally, the applied approximate equation for electric potential is valid for large distance. The dipole moment of water molecule is $\mathrm{p}=1.85 \mathrm{D}$ and the equivalent displacement is $\mathrm{d}=\mathrm{p} / \mathrm{q}=$ $1.85 \times\left(3.33564 \times 10^{-30} \mathrm{C} \cdot \mathrm{m}\right) /\left(0.82 \times 1.6 \times 10^{-19} \mathrm{C}\right)=4.7 \times 10^{-11} \mathrm{~m}=0.47 \AA \quad$ given that $\mathrm{qO}=-0.82 \mathrm{e}$. In the calculation of interaction potential for water, the cut-off distance of $\mathrm{z}=2.5 \AA$, which is more than 5 times of the displacement of the dipole. Therefore, the expression used for electric potential of dipole moment is considered as an acceptable approximation even for the minimum separation. Meanwhile, the accuracy of the expression will increase as the adsorbate-adsorbent separation $\mathrm{z}$ increases because the error of this approximation is inverse proportional to $\mathrm{z}^{2}$, which means the rest $\mathrm{U}(\mathrm{z})$ values on the potential curves are more reliable than the acceptable $\mathrm{U}\left(\mathrm{z}_{\text {cut-off }}\right)$ [6]. Lastly, the final result reveals the fact that even for polar molecules the LJ potential is still the dominant part in the interaction potential and this also moderates the approximation of electric potential expressions. The electric potential of elementary charge in the electric field excited by a permanent dipole $\boldsymbol{\mu}$ at the distance $\mathbf{r}$ is,

$$
\phi_{\text {dipole }}(\mathbf{r})=\frac{1}{4 \pi \varepsilon_{0}} \frac{\mathbf{r} \cdot \boldsymbol{\mu}}{\mathbf{r}^{3}}
$$

The electric field excited by a permanent dipole $\boldsymbol{\mu}$ at the distance $\mathbf{r}$ is,

$$
\mathbf{E}_{\text {dipole }}(\mathbf{r})=-\nabla \phi_{\text {dipole }}(\mathbf{r})=\frac{1}{4 \pi \varepsilon_{0}}\left(\frac{3(\boldsymbol{\mu} \cdot \mathbf{r}) \mathbf{r}}{\mathbf{r}^{5}}-\frac{\boldsymbol{\mu}}{\mathbf{r}^{3}}\right)
$$

\section{Potential of quadrupole in static electric field}

The potential of a quadrupole in electric field $\mathbf{F}$ is the double point product of two tensors,

$$
\mathrm{U}=-\frac{1}{3} \Theta: \nabla \mathbf{F},
$$


where $\boldsymbol{\Theta}$ is the quadrupole moment tensor and $\boldsymbol{\nabla F}$ is the EFG tensor.

\section{Electric field generated by a quadrupole}

The electric potential of elementary charge in the electric field excited by a quadrupole $\boldsymbol{\Theta}$ at the distance $\mathbf{r}$ is,

$$
\phi_{\text {quadrupole }}(\mathbf{r})=\frac{1}{4 \pi \varepsilon_{0}} \frac{1}{2} \frac{\mathbf{r}^{\mathbf{T}} \cdot \boldsymbol{\Theta} \cdot \mathbf{r}}{\mathbf{r}^{5}}
$$

The electric field excited by a quadrupole $\boldsymbol{\Theta}$ at the distance $\mathbf{r}$ is,

$$
\mathbf{E}_{\text {quadrupole }}(\mathbf{r})=-\nabla \phi_{\text {quadrupole }}(\mathbf{r})=\frac{1}{4 \pi \varepsilon_{0}}\left(\frac{\left(5 \mathbf{r}^{\mathbf{T}} \cdot \boldsymbol{\Theta} \cdot \mathbf{r}\right) \mathbf{r}}{2 \mathbf{r}^{7}}-\frac{\boldsymbol{\Theta} \cdot \mathbf{r}}{\mathbf{r}^{5}}\right)
$$

The EFG tensor of quadrupole $\boldsymbol{\Theta}$ at the distance $\mathbf{r}$ is

$$
\operatorname{EFG}(\mathbf{r})=\nabla \mathbf{E}_{\text {quadrupole }}(\mathbf{r})=\frac{1}{4 \pi \varepsilon_{0}}\left(\frac{\left(5 \mathbf{r}^{\mathbf{T}} \cdot \boldsymbol{\Theta} \cdot \mathbf{r}\right) \mathbf{I}}{2 \mathbf{r}^{7}}+\frac{\boldsymbol{\Theta}}{\mathbf{r}^{5}}\right)
$$

where $\mathbf{r}^{\mathbf{T}}$ is the transposed position vector, $\mathbf{I}$ is a $3 \times 3$ unit diagonal matrix.

\section{A4. Computational Strategy}

The computational strategy for electric field generated by both molecules of this simulation is shown in Table A1 
Table 4A1 The computation of electric field with induction effect for a pair of molecules Step 0: Initiate electric fields

$$
\begin{aligned}
& \mathbf{E}_{\mathbf{m}}(-\mathbf{r})=\mathbf{E}_{\mathbf{m}}^{0}(-\mathbf{r})=\frac{1}{4 \pi \varepsilon_{0}}\left(\frac{\mu_{\mathbf{m}}}{\mathbf{r}^{3}}-\frac{3\left(\mu_{\mathbf{m}} \cdot \mathbf{r}\right) \mathbf{r}}{\mathbf{r}^{5}}\right)+\frac{1}{4 \pi \varepsilon_{0}}\left(\frac{\left(5 \mathbf{r}^{\mathrm{T}} \cdot \boldsymbol{\Theta}_{\mathbf{m}} \cdot \mathbf{r}\right) \mathbf{r}}{2 \mathbf{r}^{7}}-\frac{\boldsymbol{\Theta}_{\mathbf{m}} \cdot \mathbf{r}}{\mathbf{r}^{5}}\right) \\
& \mathbf{E}_{\mathbf{M}}(\mathbf{r})=\mathbf{E}_{\mathbf{M}}^{0}(\mathbf{r})=\frac{1}{4 \pi \varepsilon_{0}}\left(\frac{\left(5 \mathbf{r}^{\mathbf{T}} \cdot \boldsymbol{\Theta}_{\mathbf{M}} \cdot \mathbf{r}\right) \mathbf{r}}{2 \mathbf{r}^{7}}-\frac{\boldsymbol{\Theta}_{\mathbf{M}} \cdot \mathbf{r}}{\mathbf{r}^{5}}\right) \\
& \mathbf{E}_{\mathbf{m}}^{\mathrm{I}}(-\mathbf{r})=\left[\mathbf{E}_{\mathbf{m}}^{\mathrm{I}}(-\mathbf{r})\right]^{0}=0 \\
& \mathbf{E}_{\mathbf{M}}^{\mathrm{I}}(\mathbf{r})=\left[\mathbf{E}_{\mathbf{M}}^{\mathrm{I}}(\mathbf{r})\right]^{0}=0 \\
& i=1
\end{aligned}
$$

Step 1:Update electric fields

$$
\begin{aligned}
& \mathbf{E}_{\mathbf{m}}^{\mathrm{i}}(-\mathbf{r})=\mathbf{E}_{\mathbf{m}}^{0}(-\mathbf{r})+\mathbf{E}_{\mathbf{m}}^{\mathrm{I}}(-\mathbf{r}) \\
& \mathbf{E}_{\mathbf{M}}^{\mathrm{i}}(\mathbf{r})=\mathbf{E}_{\mathbf{M}}^{0}(\mathbf{r})+\mathbf{E}_{\mathbf{M}}^{\mathrm{I}}(\mathbf{r})
\end{aligned}
$$

Step2: Calculate induced dipoles

$$
\begin{aligned}
& \boldsymbol{\mu}_{\mathrm{m}}^{\mathrm{I}}=\boldsymbol{\alpha}_{\mathrm{m}} \cdot \mathbf{E}_{\mathrm{M}}^{\mathrm{i}}(\mathbf{r}) \\
& \boldsymbol{\mu}_{\mathrm{M}}^{\mathrm{I}}=\boldsymbol{\alpha}_{\mathrm{M}} \cdot \mathbf{E}_{\mathrm{m}}^{\mathrm{i}}(-\mathbf{r})
\end{aligned}
$$

Step3: Update electric fields of induced dipoles

$$
\begin{aligned}
& \mathbf{E}_{\mathbf{m}}^{\mathrm{I}}(-\mathbf{r})=\left[\mathbf{E}_{\mathbf{m}}^{\mathrm{I}}(-\mathbf{r})\right]^{\mathrm{i}}=\frac{1}{4 \pi \varepsilon_{0}}\left(\frac{\boldsymbol{\mu}_{\mathrm{m}}^{\mathrm{I}}}{\mathbf{r}^{3}}-\frac{3\left(\boldsymbol{\mu}_{\mathrm{m}}^{\mathrm{I}} \cdot \mathbf{r}\right) \mathbf{r}}{\mathbf{r}^{5}}\right) \\
& \mathbf{E}_{\mathbf{M}}^{\mathrm{I}}(\mathbf{r})=\left[\mathbf{E}_{\mathbf{M}}^{\mathrm{I}}(\mathbf{r})\right]^{\mathrm{i}}=\frac{1}{4 \pi \varepsilon_{0}}\left(\frac{3\left(\boldsymbol{\mu}_{\mathbf{M}}^{\mathrm{I}} \cdot \mathbf{r}\right) \mathbf{r}}{\mathbf{r}^{5}}-\frac{\boldsymbol{\mu}_{\mathbf{M}}^{\mathrm{I}}}{\mathbf{r}^{3}}\right)
\end{aligned}
$$

Step 4: Iteration*

$$
\text { if }\left(\left|\mathbf{E}_{\mathbf{m}}^{\mathrm{i}}(-\mathbf{r})-\mathbf{E}_{\mathbf{m}}^{\mathrm{i}-1}(-\mathbf{r})\right| /\left|\mathbf{E}_{\mathbf{m}}^{\mathrm{i}-1}(-\mathbf{r})\right|<\mathbf{e} \text { and }\left|\mathbf{E}_{\mathbf{M}}^{\mathrm{i}}(\mathbf{r})-\mathbf{E}_{\mathbf{M}}^{\mathrm{i}-1}(\mathbf{r})\right| /\left|\mathbf{E}_{\mathbf{M}}^{\mathrm{i}-1}(\mathbf{r})\right|<\mathbf{e}\right)
$$

Stop computation

$$
\text { else }
$$

$$
\mathrm{i}=\mathrm{i}+1 \text { and Go to Step } 1
$$

*The total number of iterations can be defined to stop the computation as long as the difference of electric field calculated in two consecutive iterations satisfies the convergence criterion $\boldsymbol{e}$. 
The sum of electric fied of all pairs of the adsorbate and adsorbent molecules can be used to calculate the induction potential of the whole system.

The computational strategy for interaction potential of one adsorbate molecule and the multilayers of carbon atoms is shown in Table A2

Table A2 The computation of interaction potential of one adsorbate molecule and multilayer carbon atoms

Step 0: Initiate position and orientation of adsorbate molecule

$$
\begin{aligned}
& \mathbf{r}_{\mathbf{M}}=\mathbf{T}_{\boldsymbol{\alpha}, \boldsymbol{\beta}, \boldsymbol{\gamma}} \mathbf{r}_{\mathbf{m}}+\mathbf{r}_{\mathbf{m}, \mathbf{0}} * \\
& \mathrm{U}_{\mathrm{mM}}=\mathrm{U}_{\mathrm{LJ}}^{0}=\mathrm{U}_{\mathrm{E}}^{0}=\mathrm{U}_{\mathrm{I}}^{0}=0 \\
& \mathrm{i}=1
\end{aligned}
$$

Step 1:Update electric field, EFG and induced dipole moment

Update $\mathbf{E}_{\mathbf{m}}^{\mathrm{i}}\left(-\mathbf{r}_{\mathbf{M}}\right), \mathbf{E}_{\mathbf{M}}^{\mathrm{i}}\left(\mathbf{r}_{\mathbf{M}}\right), \boldsymbol{\mu}_{\mathbf{m}}^{\mathrm{I}}$ and $\boldsymbol{\mu}_{\mathbf{M}}^{\mathrm{I}}$ according to Table 1

$$
\mathbf{E F G}_{\mathbf{M}}^{\mathrm{i}}\left(\mathbf{r}_{\mathbf{M}}\right)=\frac{1}{4 \pi \varepsilon_{0}}\left(\frac{\left(5 \mathbf{r}_{\mathbf{M}}{ }^{\mathbf{T}} \cdot \boldsymbol{\Theta} \cdot \mathbf{r}_{\mathbf{M}}\right) \mathbf{I}}{2 \mathbf{r}_{\mathbf{M}}{ }^{7}}+\frac{\boldsymbol{\Theta}}{\mathbf{r}_{\mathbf{M}}{ }^{5}}\right)
$$

Step2: Calculate interaction potential

$$
\begin{aligned}
& \mathrm{U}_{\mathrm{LJ}}^{\mathrm{i}}=\mathrm{U}_{\mathrm{LJ}}^{\mathrm{i}-1}+\sum \mathrm{U}_{\mathrm{LJ}}\left(\mathbf{r}_{\mathbf{M}}\right) * * \\
& \mathrm{U}_{\mathrm{E}}^{\mathrm{i}}=\mathrm{U}_{\mathrm{E}}^{\mathrm{i}-1}+\boldsymbol{\mu}_{\mathbf{m}} \mathbf{E}_{\mathbf{M}}^{\mathrm{i}}\left(\mathbf{r}_{\mathbf{M}}\right)+\frac{1}{3} \boldsymbol{\Theta}_{\mathbf{m}}: \mathbf{E F G}_{\mathbf{M}}^{\mathrm{i}}\left(\mathbf{r}_{\mathbf{M}}\right) \\
& \mathrm{U}_{\mathrm{I}}^{\mathrm{i}}=\mathrm{U}_{\mathrm{I}}^{\mathrm{i}-1}-\frac{1}{2} \boldsymbol{\mu}_{\mathbf{m}}^{\mathrm{I}} \mathbf{E}_{\mathbf{M}}^{\mathrm{i}}\left(\mathbf{r}_{\mathbf{M}}\right)-\frac{1}{2} \boldsymbol{\mu}_{\mathbf{M}}^{\mathrm{I}} \mathbf{E}_{\mathbf{m}}^{\mathrm{i}}\left(-\mathbf{r}_{\mathbf{M}}\right)
\end{aligned}
$$

Step 3: Iteration util I reaches the total number of carbon atoms

$$
\begin{aligned}
& \text { if }(\mathbf{i}>\mathbf{N}) \\
& U_{m M}=U_{L J}^{N}+U_{E}^{N}+U_{I}^{N}
\end{aligned}
$$

Stop computation

else

$$
\mathrm{i}=\mathrm{i}+1 \text { and Go to Step } 1
$$

* See 9. Coordinate transformation 
**eq (4.1) in main text is applied to each atom of adsorbate molecule with corresponding

position vectors and the sum will be the $\mathrm{LJ}$ part of interaction potential between one adsorbate molecule and one carbon atom.

The computational strategy for isosteric heat of adsorption at zero surface coverage is shown in

\section{Table A3}

Table A3 The computation of isosteric heat of adsorption at zero surface coverage

Step 0: Initialize constant and parameters

$\mathrm{k}_{B}, \mathrm{~T}$, integration method and precision, etc

Step 1: Initialize interaction potential function*

$$
\begin{aligned}
& \mathrm{U}_{\mathrm{mM}}(\mathrm{z})=\left.\mathrm{U}_{\mathrm{mM}}(\mathbf{r}(\mathrm{z}))\right|_{\mathrm{x}_{0}, \mathrm{y}_{0}}=\mathrm{U}_{\mathrm{mM}}\left(\mathrm{x}_{0}, \mathrm{y}_{0}, \mathrm{z}\right) \\
& \mathrm{U}_{\mathrm{mM}}(\mathrm{H}-\mathrm{z})=\left.\mathrm{U}_{\mathrm{mM}}(\mathbf{r}(\mathrm{H}-\mathrm{z}))\right|_{\mathrm{x}_{0}, \mathrm{y}_{0}}=\mathrm{U}_{\mathrm{mM}}\left(\mathrm{x}_{0}, \mathrm{y}_{0}, \mathrm{H}-\mathrm{z}\right)
\end{aligned}
$$

Step 2: Initialize external wall potential

$$
\mathrm{V}_{\mathrm{ext}}(\mathrm{z})=\mathrm{U}_{\mathrm{mM}}(\mathrm{z})+\mathrm{U}_{\mathrm{mM}}(\mathrm{H}-\mathrm{z})=\mathrm{U}_{\mathrm{mM}}\left(\mathrm{x}_{0}, \mathrm{y}_{0}, \mathrm{z}\right)+\mathrm{U}_{\mathrm{mM}}\left(\mathrm{x}_{0}, \mathrm{y}_{0}, \mathrm{H}-\mathrm{z}\right)
$$

Step 3: Integerate for numerator

$$
f_{\text {nume }}(\mathrm{H})=\int_{0}^{H} \mathrm{~V}_{\text {ext }}(\mathrm{z}) e^{-\frac{\mathrm{V}_{\text {ext }}(\mathrm{z})}{\mathrm{k}_{B} \mathrm{~T}}} d z
$$

Step 4: Integrate for denominator

$$
f_{\text {denomi }}(\mathrm{H})=\int_{0}^{H} e^{-\frac{\mathrm{v}_{\text {ext }}(\mathrm{z})}{\mathrm{k}_{B} \mathrm{~T}}} d z
$$

Step 5: Calculate isosteric heat of adsorption

$$
q_{s t}^{o}(\mathrm{H})=\mathrm{k}_{B} \mathrm{~T}-\frac{\int_{0}^{H} \mathrm{~V}_{\text {ext }}(\mathrm{z}) e^{-\frac{\mathrm{V}_{\text {ext }}(\mathrm{z})}{\mathrm{k}_{B} \mathrm{~T}}} d z}{\int_{0}^{H} e^{-\frac{\mathrm{V}_{\text {ext }}(\mathrm{z})}{\mathrm{k}_{B} \mathrm{~T}}} d z}=\mathrm{k}_{B} \mathrm{~T}-\frac{f_{\text {nume }}(\mathrm{H})}{f_{\text {denomi }}(\mathrm{H})}
$$

Stop computation.

* The interaction potential as a function of separation distance $\mathrm{z}$ is obtained by interpolation method from simulation data provided in Table A2

\section{A5. Dipole-quadrupole Interaction Potential}

Non-zero dipole and quadrupole moment exists in the interaction pair of molecule (denoted by $\mathbf{m}$ and $\mathbf{M}$, respectively), the dipole-quadrupole interaction needs to be taken into consideration in 
the calculation of interaction potential. The interaction effect may decompose into three steps, i.e.,

firstly, the generation of electric field by the dipole and quadrupole,

secondly, the deriving of interaction potential of dipole and quadrupole in the generated static electric field (electrostatic potential),

thirdly, the deriving of interaction potential of induced dipole in the generated static electric field (induction potential).

Therefore, the dipole-quadrupole interaction consist of electrostatic potential $\mathrm{u}_{\mathrm{E}}$ and induction potential $\mathrm{u}_{\mathrm{I}}$. The electrostatic potential is,

$$
\begin{aligned}
\mathrm{U}_{\mathrm{E}} & =\mathrm{U}_{\text {dipole }, \mathrm{E}(\text { quarupole } \rightarrow \text { dipole })}+\mathrm{U}_{\text {quadrupole }, \mathrm{E}(\text { dipole } \rightarrow \text { quarupole })} \\
& =\boldsymbol{\mu}_{\mathbf{m}} \cdot \mathbf{E}_{\mathbf{M}}(\mathbf{r})+\frac{1}{3} \boldsymbol{\Theta}_{\mathbf{m}} \cdot \boldsymbol{\nabla} \mathbf{E}_{\mathbf{M}}(\mathbf{r})
\end{aligned}
$$

where $\mathbf{r}$ is the distance vector from quadrupole to dipole, $\mathbf{E}_{\mathbf{M}}(\mathbf{r})$ is the electric filed at the dipole generated by the quadrupole, $\nabla \mathbf{E}_{\mathbf{M}}(\mathbf{r})$ is the $\mathbf{E F G}$ of $\mathbf{E}_{\mathbf{M}}(\mathbf{r})$. Considering that the molecule $M$ with only non-zero quadrupole moment can still induce dipole in static electric field, both induction effects should be added to the total induction potential during deriving $\mathrm{U}_{\mathrm{I}}$. The induction potential is

$$
\begin{aligned}
\mathrm{U}_{\mathrm{I}} & =\mathrm{U}_{\text {dipole, } \mathrm{E}(\text { quarupole } \rightarrow \text { dipole })}^{\mathrm{I}}+\mathrm{U}_{\text {quadrupole }, \mathrm{E}(\text { dipole } \rightarrow \text { quarupole })}^{\mathrm{I}} \\
& =-\frac{1}{2} \boldsymbol{\alpha}_{\mathrm{m}} \cdot \mathbf{E}_{\mathbf{M}}^{2}(\mathbf{r})-\frac{1}{2} \boldsymbol{\alpha}_{\mathrm{M}} \cdot \mathbf{E}_{\mathbf{m}}^{2}(-\mathbf{r})
\end{aligned}
$$

where $\boldsymbol{\alpha}_{\mathrm{m}}$ is the dipole polarizability of the molecule with non-zero dipole moment $\boldsymbol{\mu}, \boldsymbol{\alpha}_{\mathrm{M}}$ is the dipole polarizability of the molecules with non-zero quadrupole moment $\boldsymbol{\Theta}$. In consideration of the contribution of electric field generated by the induced dipole on molecules, the electric field at $\mathbf{r}$ and $-\mathbf{r}$ should be calculated iteratively. 


\section{A6. Interaction Potential of Water on Graphite}

With fixed $\mathrm{z}$, the interaction potential can vary with $\mathrm{x}$ and $\mathrm{y}$ because of the change of relative orientation of adsorbate molecule to the centre of hexagonal unit cell of graphite lattice. The distribution of interaction potential of water on graphite at orientation 1 to 5 in the simulated space is plotted as Figures A1(a) to A1(e) Six blue points represent the position of carbon atoms of hexagonal unit cell on XY plane. 


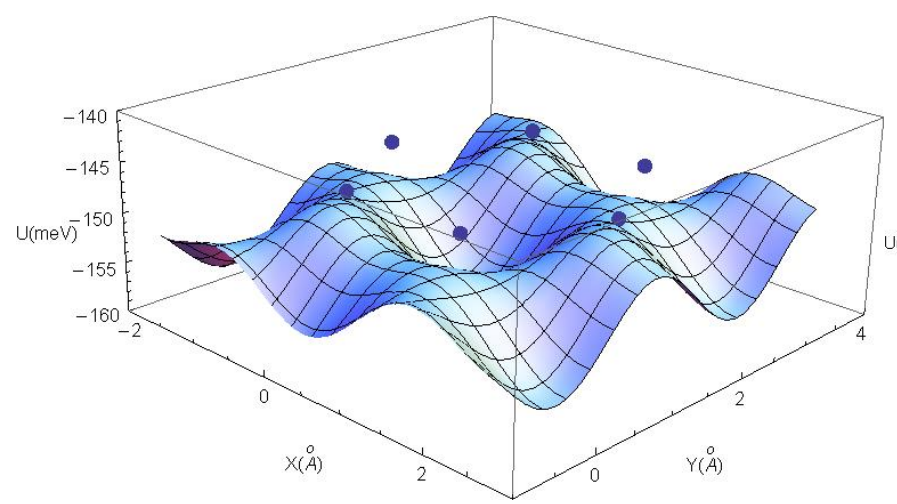

Figure A1(a) Interaction potential of water on graphite at orientation 1 and $\mathrm{Z}=3 \AA \AA$.

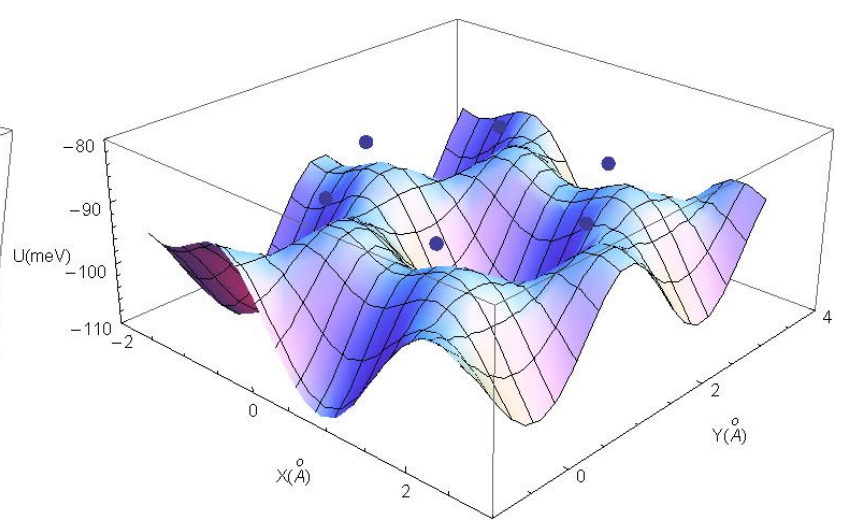

Figure A1(b) Interaction potential of water on graphite at orientation 2 and $\mathrm{Z}=3.5 \AA$.

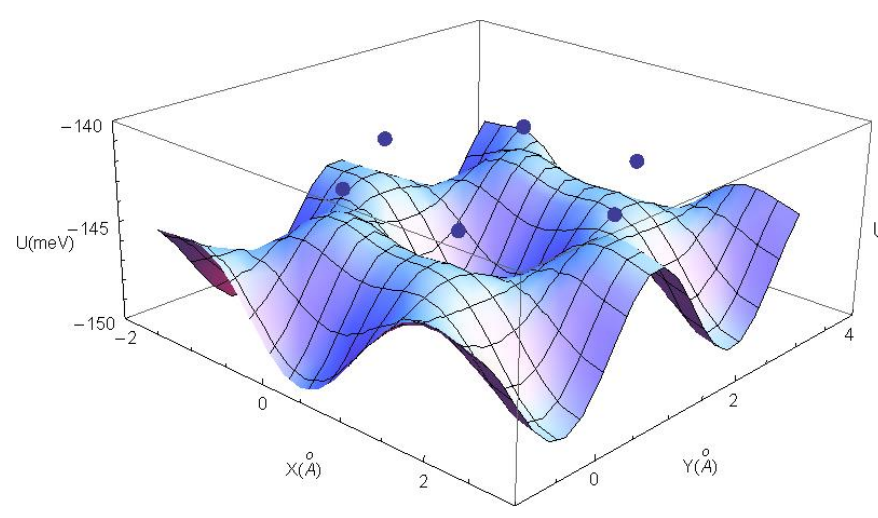

Figure A1(c) Interaction potential of water on graphite at orientation 3 and $\mathrm{Z}=3 \AA$

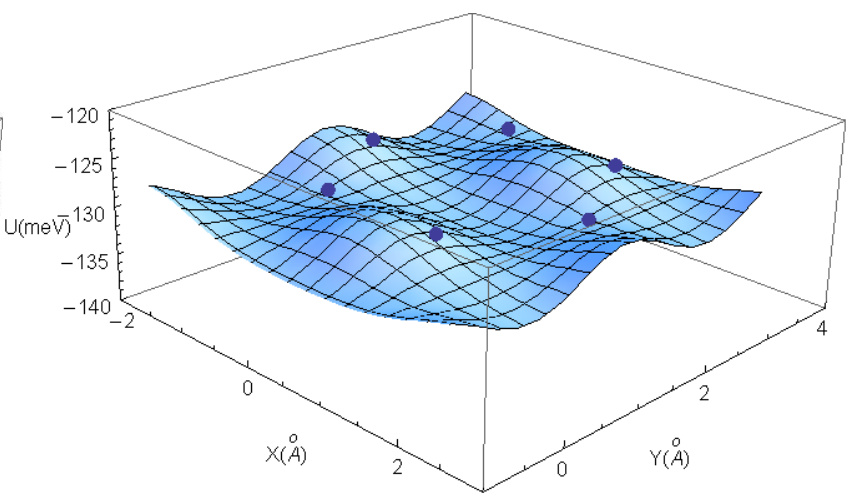

Figure A1(d) Interaction potential of water on graphite at orientation 4 and $\mathrm{Z}=3.5 \AA$.

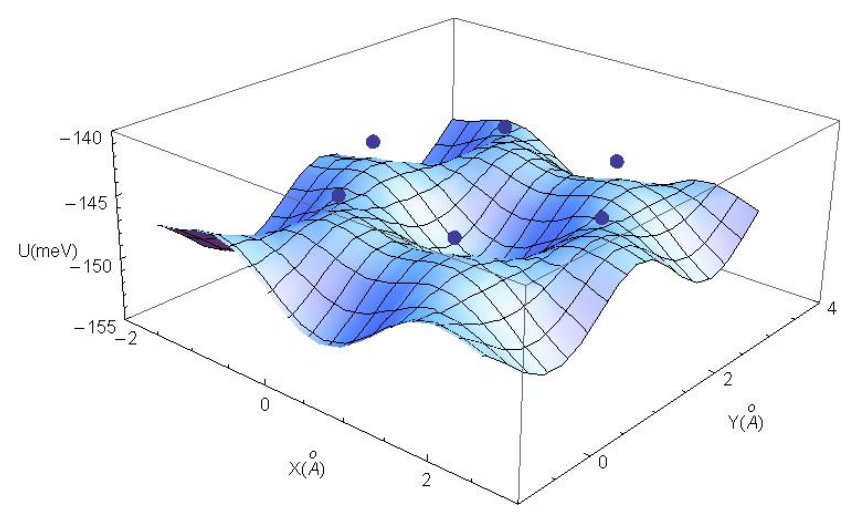

Figure A1(e) Interaction potential of water on graphite at orientation 5 and $Z=3 \AA$. 
For understanding the LJ, induction and electrostatic potentials, plots of interaction energy as a function of $\mathrm{C} \cdots \mathrm{O}$ separation distance for orientations 4 and 5 of a water molecule are shown in Figures A2(a) and A2(b).
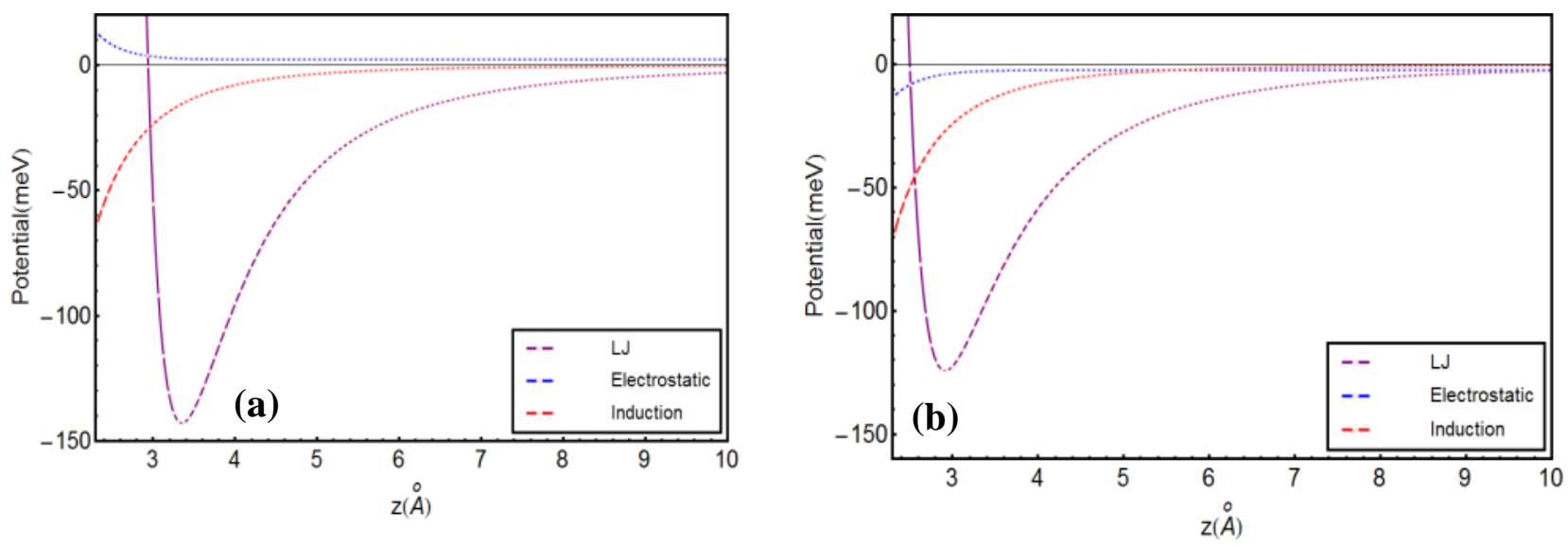

Figure A2 Water potential plots for (a) orientation 4 (b) orientation $5\left(\mathrm{U}_{\mathrm{E}}>0\right.$ for orientation 4 and $\mathrm{U}_{\mathrm{E}}<0$ for orientation 5)

\section{A7. Interaction Potential of Methanol on Graphite}

The distribution of interaction potential of methanol on graphite at orientations 1,2 and 3 in the

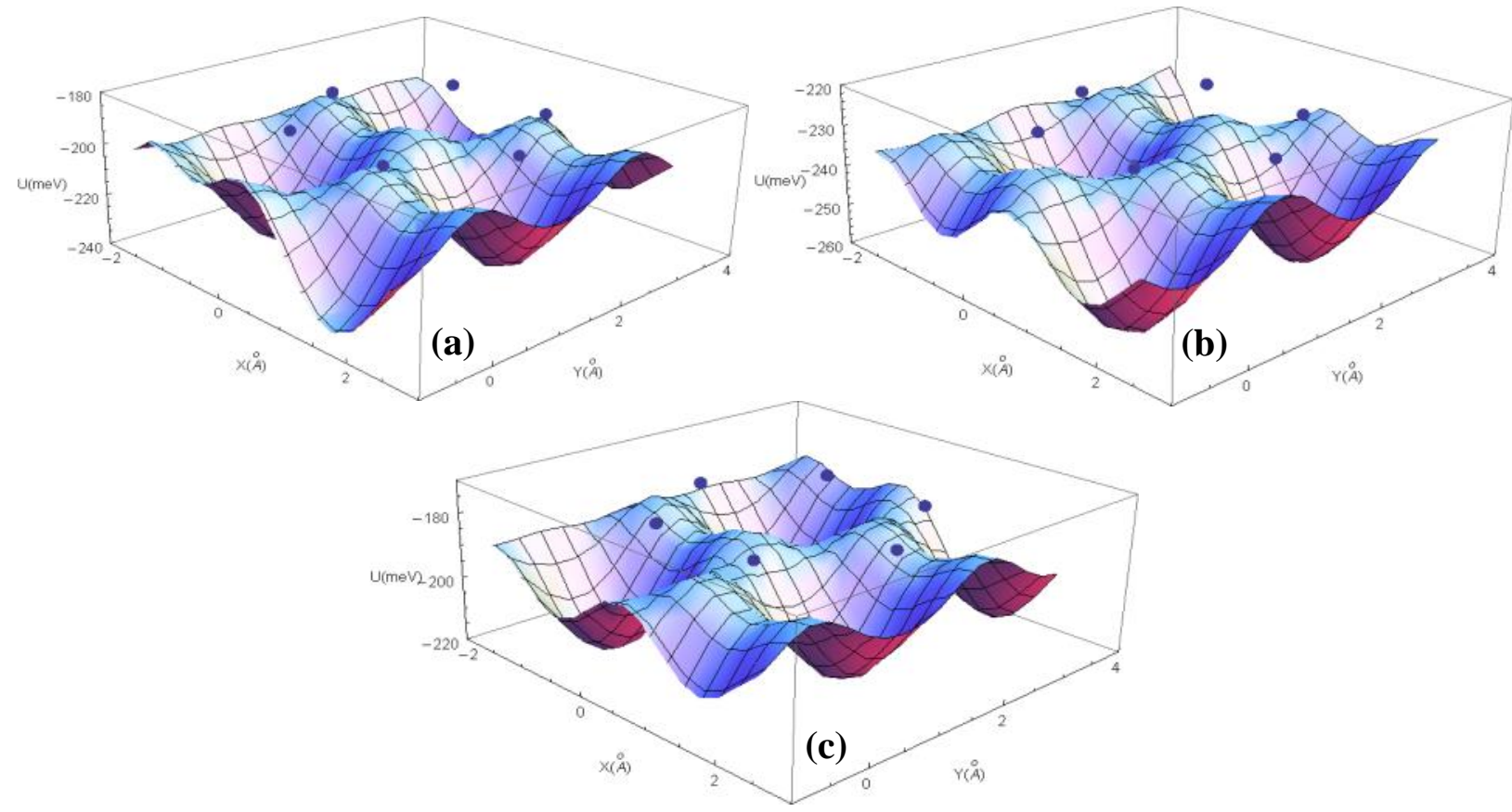

Figure A3 Interaction potentials of methanol on graphite at (a) orientation 1 for $\mathrm{Z}=3.5 \AA$, (b) orientation 2 and $\mathrm{Z}=3.5 \AA$, and (c) orientation 3 and $\mathrm{Z}=3.5 \AA$. 
simulated space are plotted in Figures A3(a), A3(b) and A3(c) Six blue points represent the position of carbon atoms of hexagonal unit cell on XY plane.

For better understanding the LJ, induction and electrostatic potentials separately, a plot of interaction energy as a function of $\mathrm{C} \cdots \mathrm{CH}_{3}-\mathrm{OH}$ separation distance for orientation 3 of methanol molecule is shown in Figure A4

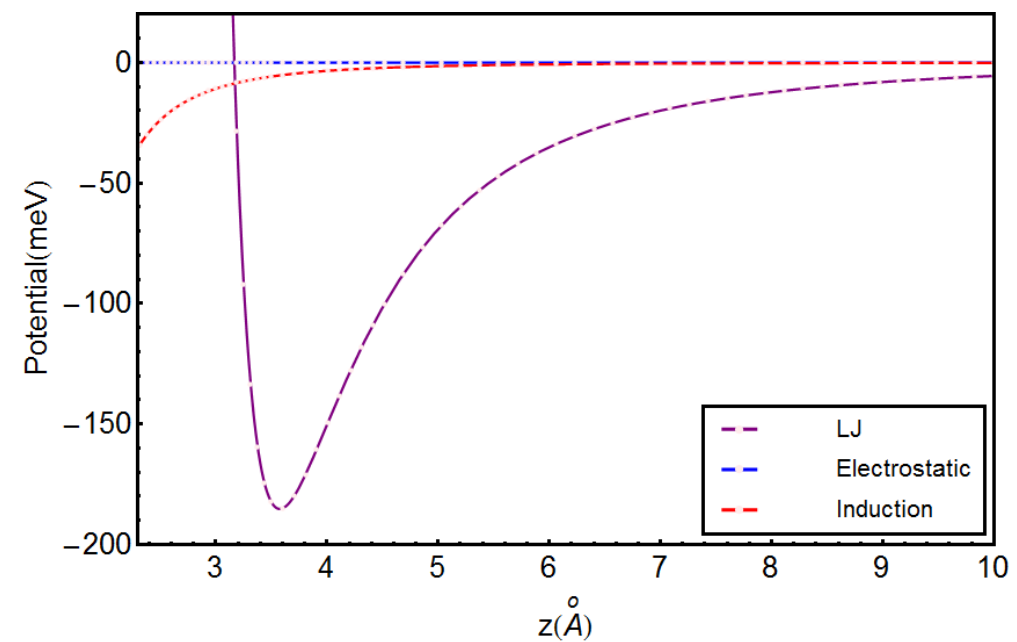

Figure A4 Various interaction energy for methanol and graphite system at orientation 3

$\left(\mathrm{U}_{\mathrm{E}}<0\right.$ for all of the 3 orientations $)$

\section{A8. Interaction Potential of Ethanol on Graphite}

The distribution of interaction potential of ethanol on graphite ranging from orientations 1 to 3 in the simulated space is plotted in Figures A5(a), A5(b) and A5(c) Six blue points represent the position of carbon atoms of hexagonal unit cell on XY plane.

The LJ, induction and electrostatic potentials are plotted as a function of $\mathrm{C} \cdots \mathrm{CH}_{3}-\mathrm{CH}_{2}-\mathrm{OH}$ separation distance for the orientation 2 of ethanol molecule is shown in Figure A6 It is observed that the electrostatic potential is very closed to zero and provides attractive force. 

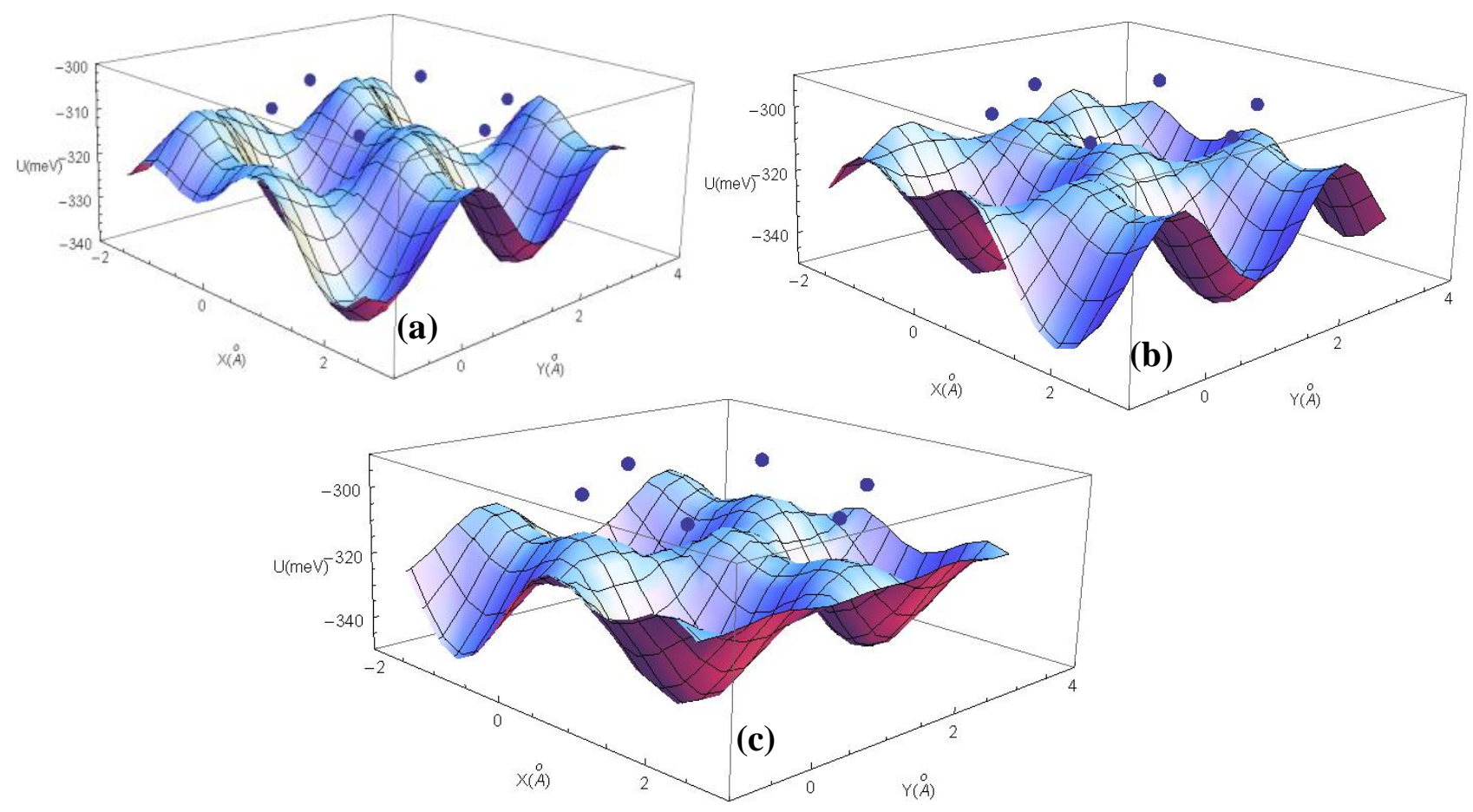

Figure A5 Interaction potential surface of ethanol on graphite for (a) orientation 1 (b) orientation 2 and (c) orientation 3 at $\mathrm{Z}=3.5 \AA$.

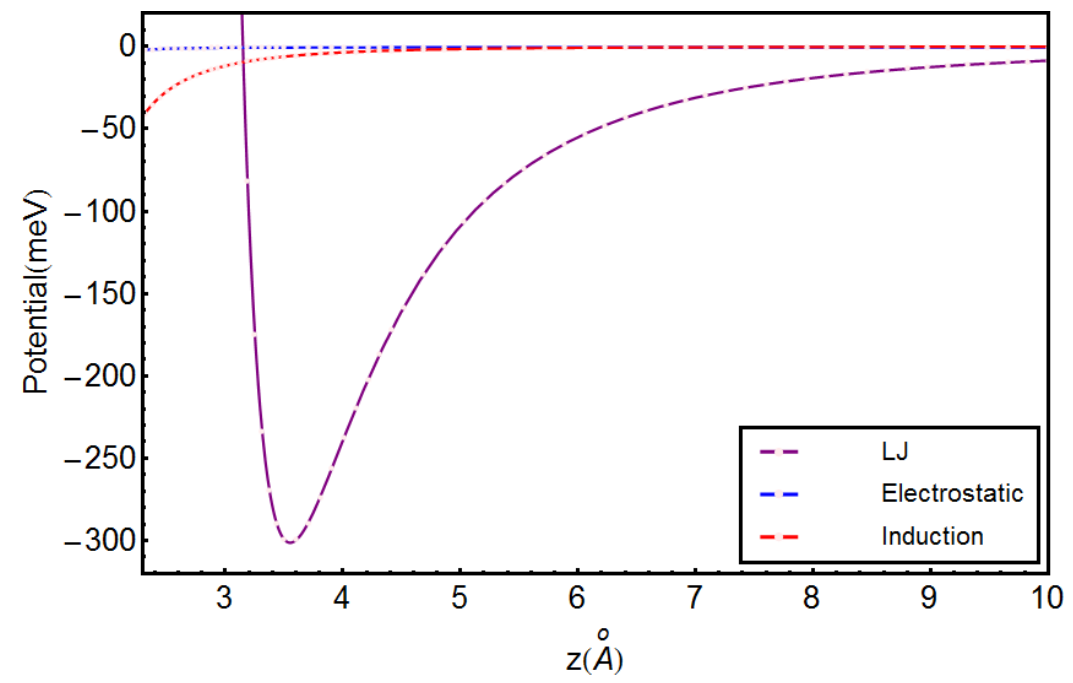

Figure A6 Ethanol potential for orientation 2 ( $\mathrm{U}_{\mathrm{E}}<0$ for all of the 3 orientations) 


\section{A9. Coordinate Transformation}

For coordinate of atoms of adsorbate molecule, the coordinate transformation from the adsorbate coordinate system $\left(\mathrm{X}^{\prime} \mathrm{Y}^{\prime} \mathrm{Z}^{\prime}\right)$ to the adsorbent (graphite) coordinate system (XYZ) can be accomplished by rotation and translation.

The column vector $\left(x^{\prime}, y^{\prime}, z^{\prime}\right)$ in $\mathrm{X}^{\prime} \mathrm{Y}^{\prime} \mathrm{Z}^{\prime}$ system is rotated in the order of around $\mathrm{X}^{\prime}, \mathrm{Y}^{\prime}$ and $\mathrm{Z}^{\prime}$ axis by $\alpha, \beta$ and $\gamma$ degree correspondingly. This temporary vector is then translated by a given translational vector $\left(x_{0}, y_{0}, z_{0}\right)$ and the final column vector $(x, y, z)$ is the transformation result from $X^{\prime} Y^{\prime} Z$ ' system to XYZ system.

$\left[\begin{array}{l}x \\ y \\ z\end{array}\right]=\left[\begin{array}{ccc}\cos \beta \cos \gamma & \cos \gamma \sin \alpha \sin \beta-\cos \alpha \sin \gamma & \cos \alpha \cos \gamma \sin \beta+\sin \alpha \sin \gamma \\ \cos \beta \sin \gamma & \sin \alpha \sin \beta \sin \gamma+\cos \alpha \cos \gamma & \cos \alpha \sin \beta \sin \gamma-\cos \gamma \sin \alpha \\ -\sin \beta & \cos \beta \sin \alpha & \cos \alpha \cos \beta\end{array}\right]\left[\begin{array}{l}x^{\prime} \\ y^{\prime} \\ z^{\prime}\end{array}\right]+\left[\begin{array}{l}x_{0} \\ y_{0} \\ z_{0}\end{array}\right]$

By combining various rotational angles and translational vectors, the coordinate of atoms of adsorbate molecules in any angle and distance to adsorbent molecules can be obtained.

\section{References}

[1] A. D. Buckingham. Permanent and Induced Molecular Moments and Long-Range Intermolecular Forces. Advances in Chemical Physics. 1967, 12: p.107-142.

[2] A. D. McLean, M. Yoshimine. Higher Polarizabilities of Linear Molecules. The Journal of Chemical Physics. 1967, 46: p.3682-3683.

[3] George Maroulis, Constantinos Makris, Demetrios Xenides, Panaghiotis Karamanis. Electric dipole and quadrupole moment and dipole polarizability of CS, SiO and SiS. Molecular Physics. 2000, 98(8): p.481-491.

[4] J. A. Pople. The Statistical Mechanics of Assemblies of Axially Symmetric Molecules. II. Second Virial Coefficients. Proceedings of the Royal Society A: Mathematical, Physical \& Engineering Sciences. 1954, 221: p.508-516.

[5] A. D. Buckingham. Molecular quadrupole moments . Quarterly Reviews, Chemical Society. 1959, 13: p.183-214.

[6] D. J. Griffiths. Introduction to Electodynamics. Prentice Hall.1999, 3rd Edition : 146. 